\title{
Coordinating Anions: (Phosphino)tetraphenylborate Ligands as New Reagents for Synthesis
}

\author{
Christine M. Thomas, Jonas C. Peters \\ Arnold and Mabel Beckman Laboratories of Chemical Synthesis, California Institute of \\ Technology, Pasadena, CA 91125
}

\section{Supporting Information}

(1) Experimental Section

(2) Additional Suzuki coupling table: The coupling of ortho-tolylboronic acid with selected aryl chlorides, iodides, and bromides.

(3) Figure 1. Fully labeled drawing of $\left\{\left[\mathrm{NEt}_{4}\right]\left[\mathrm{Ph}_{3} \mathrm{BP}^{m-\mathrm{Ph} 2}\right]\right\}_{2}$.

(4) Figure 2. Fully labeled drawing of $\left\{\left[\mathrm{NEt}_{4}\right]\left[\mathrm{Ph}_{3} \mathrm{BP}^{m-\mathrm{iPr} 2}\right]\right\}_{2}$.

(5) Figure 3. Fully labeled drawing of $\left[\mathrm{NBu}_{4}\right]\left[\left(\mathrm{Ph}_{3} \mathrm{BP}^{p-\mathrm{iPr} 2}\right) \mathrm{RhCl}(\mathrm{NBD})\right]$.

(6) Figure 4. Fully labeled drawing of $[\mathrm{ASN}]\left[\left(\mathrm{Ph}_{3} \mathrm{BP}^{m-\mathrm{Ph} 2}\right)_{2} \mathrm{PtMe}_{2}\right]$.

(7) Table 1. Crystal data and structure refinement for $\left\{\left[\mathrm{NEt}_{4}\right]\left[\mathrm{Ph}_{3} \mathrm{BP}^{m-\mathrm{Ph} 2}\right]\right\}_{2}$.

(8) Table 2. Atomic coordinates $\left(\mathrm{x} 10^{4}\right)$ and equivalent isotropic displacement parameters $\left(\AA^{2} \times 10^{3}\right)$ for $\left\{\left[\mathrm{NEt}_{4}\right]\left[\mathrm{Ph}_{3} \mathrm{BP}^{m-\mathrm{Ph} 2}\right]\right\}_{2}$.

(9) Table 3. Bond lengths $[\AA]$ and angles $\left[{ }^{\circ}\right]$ for $\left\{\left[\mathrm{NEt}_{4}\right]\left[\mathrm{Ph}_{3} \mathrm{BP}^{m-\mathrm{Ph} 2}\right]\right\}_{2}$.

(10) Table 4. Anisotropic displacement parameters $\left(\AA^{2} \times 10^{3}\right)$ for $\left\{\left[\mathrm{NEt}_{4}\right]\left[\mathrm{Ph}_{3} \mathrm{BP}^{m-}\right.\right.$ $\left.{ }^{\mathrm{Ph}}\right]$ ] 2 .

(11) Table 5: Hydrogen coordinates $\left(\mathrm{x} 10^{4}\right)$ and isotropic displacement parameters $\left(\AA^{2} \times 10^{3}\right)$ for $\left\{\left[\mathrm{NEt}_{4}\right]\left[\mathrm{Ph}_{3} \mathrm{BP}^{m-\mathrm{Ph} 2}\right]\right\}_{2}$.

(12) Table 6: Crystal data and structure refinement for $\left\{\left[\mathrm{NEt}_{4}\right]\left[\mathrm{Ph}_{3} \mathrm{BP}^{m-\mathrm{iPr} 2}\right]\right\}_{2}$.

(13) Table 7: Atomic coordinates (x 104) and equivalent isotropic displacement parameters $\left(\AA^{2} \times 10^{3}\right)$ for $\left\{\left[\mathrm{NEt}_{4}\right]\left[\mathrm{Ph}_{3} \mathrm{BP}^{m-\mathrm{iPr} 2}\right]\right\}_{2}$.

(14) Table 8: Bond lengths $[\AA]$ and angles $\left[{ }^{\circ}\right]$ for $\left\{\left[\mathrm{NEt}_{4}\right]\left[\mathrm{Ph}_{3} \mathrm{BP}^{m-\mathrm{Pr} 2}\right]\right\}_{2}$.

(15) Table 9: Anisotropic displacement parameters $\left(\AA^{2} \times 10^{3}\right)$ for $\left\{\left[\mathrm{NEt}_{4}\right]\left[\mathrm{Ph}_{3} \mathrm{BP}^{m-}\right.\right.$ $\left.\left.{ }^{i P r} 2\right]\right\}_{2}$.

(16) Table 10: Hydrogen coordinates $\left(\mathrm{x} 10^{4}\right)$ and isotropic displacement parameters $\left(\AA^{2} \times 10^{3}\right)$ for $\left\{\left[\mathrm{NEt}_{4}\right]\left[\mathrm{Ph}_{3} \mathrm{BP}^{m-\mathrm{iPr}}\right]\right\}_{2}$.

(17) Table 11. Crystal data and structure refinement for $\left[\mathrm{NBu}_{4}\right]\left[\left(\mathrm{Ph}_{3} \mathrm{BP}^{p-}\right.\right.$ $\left.{ }^{\mathrm{iPr}} \mathrm{RhCl}(\mathrm{NBD})\right]$.

(18) Table 12. Atomic coordinates $\left(\mathrm{x} \mathrm{10} 0^{4}\right)$ and equivalent isotropic displacement

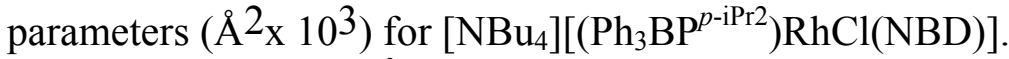

(19) Table 13. Bond lengths $[\AA]$ and angles $\left[{ }^{\circ}\right]$ for $\left[\mathrm{NBu}_{4}\right]\left[\left(\mathrm{Ph}_{3} \mathrm{BP}^{p-\mathrm{iPr} 2}\right) \mathrm{RhCl}(\mathrm{NBD})\right]$. 
(20) Table 14. Anisotropic displacement parameters $\left(\AA^{2} \times 10^{3}\right)$ for $\left[\mathrm{NBu}_{4}\right]\left[\left(\mathrm{Ph}_{3} \mathrm{BP}^{p-}\right.\right.$ $\left.\left.{ }^{\mathrm{iPr} 2}\right) \mathrm{RhCl}(\mathrm{NBD})\right]$.

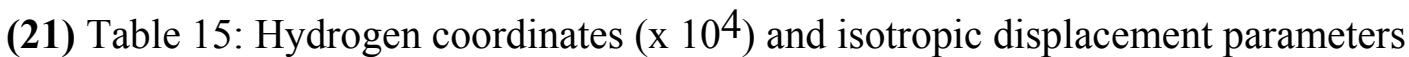
$\left(\AA^{2} \mathrm{x}_{10}{ }^{3}\right)$ for $\left[\mathrm{NBu}_{4}\right]\left[\left(\mathrm{Ph}_{3} \mathrm{BP}^{p-\mathrm{Pr} 2}\right) \mathrm{RhCl}(\mathrm{NBD})\right]$.

(22) Table 16. Crystal data and structure refinement for $[\mathrm{ASN}]\left[\left(\mathrm{Ph}_{3} \mathrm{BP}^{m-\mathrm{Ph} 2}\right)_{2} \mathrm{PtMe}_{2}\right]$.

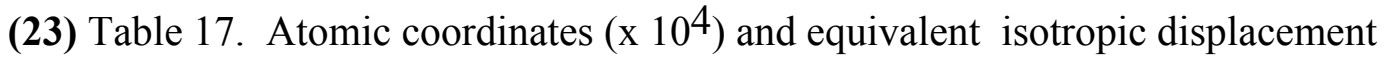
parameters $\left(\AA^{2} \times 10^{3}\right)$ for $[\mathrm{ASN}]\left[\left(\mathrm{Ph}_{3} \mathrm{BP}^{m-\mathrm{Ph} 2}\right)_{2} \mathrm{PtMe}_{2}\right]$.

(24) Table 18. Bond lengths $[\AA]$ and angles $\left[{ }^{\circ}\right]$ for $[\mathrm{ASN}]\left[\left(\mathrm{Ph}_{3} \mathrm{BP}^{m-\mathrm{Ph}_{2}}\right)_{2} \mathrm{PtMe}_{2}\right]$.

(25) Table 19. Anisotropic displacement parameters $\left(\AA^{2} \times 10^{3}\right)$ for $[\mathrm{ASN}]\left[\left(\mathrm{Ph}_{3} \mathrm{BP}^{m-}\right.\right.$ $\left.\left.{ }^{\mathrm{Ph} 2}\right)_{2} \mathrm{PtMe}_{2}\right]$.

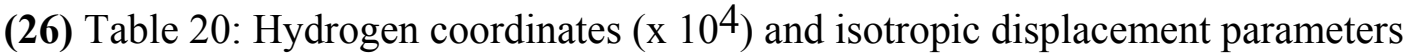
$\left(\AA^{2} \times 10^{3}\right)$ for $[\mathrm{ASN}]\left[\left(\mathrm{Ph}_{3} \mathrm{BP}^{m-\mathrm{Ph} 2}\right)_{2} \mathrm{PtMe}_{2}\right]$. 


\section{Experimental Section.}

All syntheses reported were carried out using standard glovebox and Schlenk techniques in the absence of water and dioxygen, unless otherwise noted. Acetonitrile, benzene, dichloromethane, diethyl ether, petroleum ether, tetrahydrofuran, and toluene were dried by sparging with $\mathrm{N}_{2}$ gas followed by passage through an activated alumina column. Ethanol was deoxygenated via sparging with $\mathrm{N}_{2}$, drying over $\mathrm{CaH}$, and distilling prior to use. All solvents were stored over 3-Å molecular sieves. Deuterated benzene, chloroform, acetonitrile, and acetone, were purchased from Cambridge Isotope Laboratories, Inc., degassed via repeated freeze-pump-thaw cycles, and dried over $3-\AA$ molecular sieves. Nonhalogenated solvents were frequently tested using a standard solution of sodium benzophenone ketyl in tetrahydrofuran to confirm the absence of oxygen and moisture. (COD)PtMe ${ }_{2}{ }^{1}$ (TMEDA) $\mathrm{LiCH}_{2} \mathrm{PPh}_{2}{ }^{2}$ and $\mathrm{ASNBr}^{3}$ were prepared using literature methods. $\mathrm{P}(i-\mathrm{Pr})_{2} \mathrm{Ph}$ was prepared by reaction of $(i \text { - } \mathrm{Pr})_{2} \mathrm{PCl}$ with $\mathrm{PhMgBr}$ in THF at $-90{ }^{\circ} \mathrm{C}$. (Bromophenyl)diphenylphosphine was prepared using a modification of the literature procedure as described below. ${ }^{4} \mathrm{~B}\left(\mathrm{C}_{6} \mathrm{~F}_{5}\right)_{3}$ was purchased from Strem and recrystallized from pentane at $-35{ }^{\circ} \mathrm{C}$ prior to use. All other chemicals were purchased from Aldrich, Strem, Alfa Aesar, or Lancaster and used without further purification. NMR spectra were recorded at ambient temperature on Varian Mercury $300 \mathrm{MHz}$, Inova $500 \mathrm{MHz}$, and Joel $400 \mathrm{MHz}$ spectrometers. ${ }^{1} \mathrm{H}$ and ${ }^{13} \mathrm{C}$ NMR chemical shifts were referenced to residual solvent. ${ }^{31} \mathrm{P} \mathrm{NMR}$ shifts were referenced to $85 \% \mathrm{H}_{3} \mathrm{PO}_{4}$. IR spectra were recorded on a Bio-Rad Excalibur FTS 3000 spectrometer controlled by WinIR Pro software. Elemental Analyses were performed by Desert Analytics, Tuscon, AZ. X-ray diffraction experiments were carried out by the Beckman Institute Crystallographic Facility on a Bruker Smart 1000 CCD diffractometer.

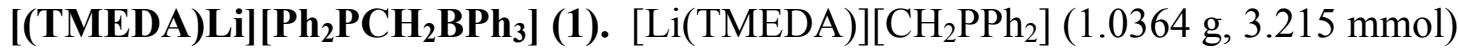
was dissolved in toluene $(18 \mathrm{~mL})$ and placed under and $\mathrm{N}_{2}$ atmosphere. To this solution was added $\mathrm{BPh}_{3}(0.778 \mathrm{~g}, 3.21 \mathrm{mmol})$ in toluene $(10 \mathrm{~mL})$ at $-78^{\circ} \mathrm{C}$. Mixture was stirred and allowed to warm to room temperature slowly over a period of 2.5 hours. Volatiles were then removed in vacuo. Solids were re-dissolved in toluene $(30 \mathrm{~mL})$ followed by filtration through a Celite plug. Volatiles was removed from the filtrate in vacuo $(\sim 90 \%$ crude yield by ${ }^{31} \mathrm{P}$ NMR). The product was recrystallized via vapor diffusion of petroleum ether into THF $(\sim 50 \%)$. ${ }^{1} \mathrm{H} \mathrm{NMR}\left(300 \mathrm{MHz}, \mathrm{d}_{6}\right.$-acetone $): \delta=7.38(\mathrm{~m}, 6 \mathrm{H})$, $\delta=7.17(\mathrm{~m}, 4 \mathrm{H}), \delta=7.02(\mathrm{~m}, 4 \mathrm{H}), \delta=7.00(\mathrm{~m}, 2 \mathrm{H}), \delta=6.83(\mathrm{~m}, 6 \mathrm{H}), \delta=6.69(\mathrm{~m}, 3 \mathrm{H})$, $\delta=3.63(\mathrm{~m}, 8 \mathrm{H}, \mathrm{THF}), \delta=1.89\left(\mathrm{~m}, 2 \mathrm{H}, \mathrm{CH}_{2}\right), \delta=1.79(\mathrm{~m}, 8 \mathrm{H}, \mathrm{THF}) .{ }^{13} \mathrm{C} \mathrm{NMR}$ $\left(75.397 \mathrm{MHz}, \mathrm{CH}_{3} \mathrm{CN}\right): \delta=135.13,132.99,132.78,127.71,126.62,125.91,122.18$, 67.83, 25.80. ${ }^{31} \mathrm{P}$ NMR (121.475 MHz, $\mathrm{d}_{6}$-acetone): -10.34 (s).

(3-Bromophenyl)diphenylphosphine (2a). 1,3-dibromobenzene (11.0 g, $47.01 \mathrm{mmol})$ was dissolved in $150 \mathrm{~mL}$ dry, degassed THF and cooled to $-95^{\circ} \mathrm{C}$ under nitrogen using an acetone/liquid nitrogen bath. ${ }^{\mathrm{n}} \mathrm{BuLi}(1.6 \mathrm{M}$ in hexane, $29.4 \mathrm{~mL}, 47.04 \mathrm{mmol})$ was added dropwise and the resulting cloudy solution was stirred at $-95^{\circ} \mathrm{C}$. After 1 hour,

\footnotetext{
${ }^{1}$ Costa, E.; Pringle, P.G.; Ravetz, M.; Inorg. Synth. 1995, 31, 284-286.

${ }^{2}$ Schore, N.E.; Benner, L.S.; Labelle, B.E. Inorg. Chem.1981, 20, 3200-3208.

${ }^{3}$ Blicke, F.F.; Hotelling, E.B. J. Am. Chem. Soc. 1954, 76, 5099-5103.

${ }^{4}$ Lustenberger, P.; Diederich, F. Helv. Chim. Acta 2000, 83, 2865-2883.
} 
chlorodiphenylphosphine $(8.43 \mathrm{~mL}, 46.98 \mathrm{mmol})$ was added dropwise. The solution was allowed to warm to room temperature over the course of 2 hours, and was then filtered through Celite in air. The resulting filtrate was dried in vacuo. The solids were then extracted with hexanes followed by filtration through a silica plug. Volatiles were removed in vacuo to afford a spectroscopically pure, moderately air-stable viscous oil $(11.70 \mathrm{~g}, 74.0 \%) .{ }^{1} \mathrm{H}$ NMR $\left(300 \mathrm{MHz}, \mathrm{d}_{6}\right.$-acetone): $\delta=7.56(\mathrm{~m}, 1 \mathrm{H}), \delta=7.41(\mathrm{~m}, 4 \mathrm{H})$, $\delta=7.25-7.40(\mathrm{~m}, 9 \mathrm{H}), 7.35 .{ }^{13} \mathrm{C} \mathrm{NMR}\left(75.397 \mathrm{MHz}, \mathrm{CH}_{3} \mathrm{CN}\right): \delta=137.45,136.55$, 134.74, 133.25, 132.78, 131.61, 130.37, 129.92, 123.87. ${ }^{31} \mathrm{P}$ NMR $\left(121.475 \mathrm{MHz}, \mathrm{d}_{6^{-}}\right.$ acetone): $\delta=-4.22(\mathrm{~s})$.

(4-Bromophenyl)diphenylphosphine (2b). 1,4-dibromobenzene (3.05 g, $13.0 \mathrm{mmol}$ ) was dissolved in $75 \mathrm{~mL}$ dry, degassed THF and cooled to $-95^{\circ} \mathrm{C}$ under nitrogen using an acetone/liquid nitrogen bath. ${ }^{\mathrm{n}} \mathrm{BuLi}(1.6 \mathrm{M}$ in hexane, $8.2 \mathrm{~mL}, 13.0 \mathrm{mmol})$ was added dropwise and the resulting cloudy solution was stirred at $-95^{\circ} \mathrm{C}$. After 1 hour, chlorodiphenylphosphine $(2.88 \mathrm{~g}, 13.0 \mathrm{mmol})$ was added dropwise. The solution was allowed to warm to room temperature over the course of 2 hours, and was then filtered through Celite under air. The filtrate was then dried in vacuo. The resulting solids were extracted with hexanes followed by filtration through a silica plug. Volatiles were then removed in vacuo to afford a spectroscopically pure, moderately air-stable viscous oil (3.060 g, $69 \%) .{ }^{1} \mathrm{H}$ NMR $\left(300 \mathrm{MHz}, \mathrm{d}_{6}\right.$-acetone): $\delta=7.52(\mathrm{~m}, 2 \mathrm{H}), \delta=7.33-7.37(\mathrm{~m}$, $6 \mathrm{H}), \delta=7.26(\mathrm{~m}, 4 \mathrm{H}), 7.16(\mathrm{~m}, 2 \mathrm{H}) .{ }^{13} \mathrm{C}$ NMR $(75.397 \mathrm{MHz}, \mathrm{THF}): \delta=137.28,137.04$, 135.38, 133.72, 131.67, 128.66, 128.94, 123.26. ${ }^{31} \mathrm{P}$ NMR (121.475 MHz, $\mathrm{d}_{6}$-acetone): $\delta$ $=-5.70(\mathrm{~s})$.

(3-Bromophenyl)diisopropylphosphine (2c). 1,3-dibromobenzene (4.2 g, $17.95 \mathrm{mmol})$ was dissolved in $150 \mathrm{~mL}$ dry, degassed THF and cooled to $-95{ }^{\circ} \mathrm{C}$ under nitrogen using an acetone/liquid nitrogen bath. ${ }^{\mathrm{n}} \mathrm{BuLi}(1.6 \mathrm{M}$ in hexane, $11.2 \mathrm{~mL}, 17.95 \mathrm{mmol})$ was added dropwise and the resulting cloudy solution was stirred at $-95{ }^{\circ} \mathrm{C}$. After 1 hour, chlorodiisopropylphosphine $(2.8 \mathrm{~mL}, 17.7 \mathrm{mmol})$ was added dropwise. The solution was allowed to warm to room temperature over the course of 2 hours, and was then filtered through Celite under a nitrogen atmosphere. The resulting filtrate was dried in vacuo and the remaining solids were extracted with petroleum ether followed by filtration through a silica plug. The volatiles were then removed in vacuo to afford the spectroscopically pure product as a viscous oil $(2.95 \mathrm{~g}, 60.6 \%)$. ${ }^{1} \mathrm{H}$ NMR (300 MHz, $\mathrm{d}_{6}$-acetone): $\delta=7.63$ $(\mathrm{m}, 1 \mathrm{H}), \delta=7.57(\mathrm{~m}, 1 \mathrm{H}), \delta=7.50(\mathrm{~m}, 1 \mathrm{H}), 7.35(\mathrm{t}, 1 \mathrm{H}, \mathrm{J}=7.6 \mathrm{~Hz}), \delta=2.15(\mathrm{~m}, 2 \mathrm{H}), \delta$ $=1.07(\mathrm{~m}, 6 \mathrm{H}), 0.90(\mathrm{~m}, 6 \mathrm{H}) \cdot{ }^{13} \mathrm{C}$ NMR $(75.397 \mathrm{MHz}, \mathrm{THF}): \delta=137.31,134.95$, 133.55, 132.24, 129.98, 122.87, 23.55, 20.20, 19.17. ${ }^{31} \mathrm{P}$ NMR (121.475 MHz, $\mathrm{d}_{6^{-}}$ acetone): $\delta=13.54$ (s).

(4-Bromophenyl)diisopropylphosphine (2d). 1,4-dibromobenzene (12.5 g, 53.42 mmol) was dissolved in $150 \mathrm{~mL}$ dry, degassed THF and cooled to $-95^{\circ} \mathrm{C}$ under nitrogen using an acetone/liquid nitrogen bath. ${ }^{\mathrm{n}} \mathrm{BuLi}(1.6 \mathrm{M}$ in hexane, $33.3 \mathrm{~mL}, 53.28 \mathrm{mmol})$ was added dropwise and the resulting cloudy solution was stirred at $-95^{\circ} \mathrm{C}$. After 1 hour, chlorodiisopropylphosphine $(8.5 \mathrm{~mL}, 53.45 \mathrm{mmol})$ was added dropwise. The solution was allowed to warm to room temperature over the course of 2 hours and was then filtered through Celite under a nitrogen atmosphere. The remaining filtrate was dried in 
vacuo and the solids were extracted with petroleum ether followed by filtration through a silica plug. Solvent was removed in vacuo yielding the spectroscopically pure product as a viscous oil $(12.03 \mathrm{~g}, 83.0 \%)$. ${ }^{1} \mathrm{H}$ NMR $\left(300 \mathrm{MHz}, \mathrm{d}_{6}\right.$-acetone $): \delta=7.51(\mathrm{~m}, 2 \mathrm{H}), \delta=$ $7.36(\mathrm{~m}, 2 \mathrm{H}), \delta=2.07(\mathrm{~m}, 2 \mathrm{H}), \delta=1.02(\mathrm{~m}, 6 \mathrm{H}), 0.84(\mathrm{~m}, 6 \mathrm{H}) .{ }^{13} \mathrm{C}$ NMR $(75.397 \mathrm{MHz}$, THF): $\delta=136.63,134.92,134.19,131.42,24.45,20.23,19.12 .{ }^{31} \mathrm{P}$ NMR $(121.475 \mathrm{MHz}$, $\mathrm{d}_{6}$-acetonitrile): $\delta=15.75(\mathrm{~s})$.

[ASN] $\left[\mathbf{P h}_{3} \mathbf{B P}^{m-\mathrm{Ph} 2}\right.$ ] (3). $m$ - $\mathrm{BrC}_{6} \mathrm{H}_{4} \mathrm{PPh}_{2}$ (2a) $(0.734 \mathrm{~g}, 2.15 \mathrm{mmol})$ was dissolved in 75 $\mathrm{mL}$ of dry, degassed THF and the stirring solution was cooled to $-90^{\circ} \mathrm{C}$. To this solution was added tert-BuLi $(1.50 \mathrm{M}$ in pentane, $1.43 \mathrm{~mL}, 2.15 \mathrm{mmol})$ and the resulting yellow solution was stirred for 1 hour at $-90^{\circ} \mathrm{C}$. Triphenylborane $(0.520 \mathrm{~g}, 2.15 \mathrm{mmol})$ was then added to the reaction pot as a THF solution. The mixture was then stirred and allowed to warm to room temperature. Volatiles were then removed in vacuo and the remaining solids were taken up in $\mathrm{CH}_{2} \mathrm{Cl}_{2}$. A solution of [ASN] $\left.\mathrm{Br}\right](0.443 \mathrm{~g}, 2.15 \mathrm{mmol})$ in $\mathrm{CH}_{2} \mathrm{Cl}_{2}$ was added dropwise. After stirring for 1 hour, copious amounts of $\mathrm{Et}_{2} \mathrm{O}$ were added to precipitate the product. The resulting solids were collected on a sintered glass frit and washed with $\mathrm{Et}_{2} \mathrm{O}$ and dried thoroughly to afford analytically pure 3 (1.175 g, 87 $\%)$. Crystals suitable for an X-ray diffraction study were grown by vapor diffusion of $\mathrm{Et}_{2} \mathrm{O}$ into acetonitrile. ${ }^{1} \mathrm{H}$ NMR $\left(300 \mathrm{MHz}, \mathrm{d}_{3}\right.$-acetonitrile): $\delta=7.50(\mathrm{~m}, 1 \mathrm{H}), \delta=7.20$ $7.32(\mathrm{~m}, 19 \mathrm{H}), \delta=6.98(\mathrm{~m}, 6 \mathrm{H}), \delta=6.83(\mathrm{~m}, 3 \mathrm{H}), \delta=3.83(\mathrm{~m}, 8 \mathrm{H}), \delta=2.10(\mathrm{~m}, 8 \mathrm{H})$. ${ }^{13} \mathrm{C}$ NMR $\left(75.396 \mathrm{MHz}, \mathrm{CH}_{3} \mathrm{CN}\right): \delta=163,142.51,139.36,136.96,135.94,133.62$, $132.38,128.68,128.55,127.98,126.04,122.24,65.10,22.19 .{ }^{31} \mathrm{P}$ NMR $(121.475 \mathrm{MHz}$, $\mathrm{d}_{3}$-acetonitrile): $\delta=\delta=-0.829$ (s). ${ }^{11} \mathrm{~B}$ NMR (400 MHz, $\mathrm{d}_{3}$-acetonitrile): $\delta=-10.99$ (s). Anal. Calcd. for $\mathrm{C}_{44} \mathrm{H}_{45} \mathrm{BNP}$ : C, 83.93; H, 6.84; N, 2.61. Found: C, 83.64; H, 7.03; N, 2.41 .

$\left[\mathbf{N B u}_{4}\right]\left[\mathbf{P h}_{3} \mathbf{B} \mathbf{P}^{p-P h 2}\right]$ (4). $p$ - $\mathrm{BrC}_{6} \mathrm{H}_{4} \mathrm{PPh}_{2}$ (2b) $(1.254 \mathrm{~g}, 3.68 \mathrm{mmol})$ was dissolved in 75 $\mathrm{mL}$ of dry, degassed THF and the stirring solution was then cooled to $-90^{\circ} \mathrm{C}$. To this solution was added tert-BuLi (1.50 M in pentane, $2.45 \mathrm{~mL}, 3.68 \mathrm{mmol})$ to form a yellow solution that was stirred for 1 hour at $-90^{\circ} \mathrm{C}$. Triphenylborane $(0.890 \mathrm{~g}, 3.68 \mathrm{mmol})$ was added as a THF solution after 1 hour and the resulting solution was stirred while warming gradually to room temperature. The reaction volatiles were then removed in vacuo and the remaining solids were taken up in $\mathrm{CH}_{2} \mathrm{Cl}_{2}$ A solution of $\left[\mathrm{NBu}_{4}\right][\mathrm{Br}](1.186 \mathrm{~g}, 3.68$ mmol) in $\mathrm{CH}_{2} \mathrm{Cl}_{2}$ was added dropwise. After 1 hour, copious amounts of $\mathrm{Et}_{2} \mathrm{O}$ were added and the solution was cooled to $-35{ }^{\circ} \mathrm{C}$ to precipitate the product, which was isolated and dried to afford spectroscopically pure $4(1.584 \mathrm{~g}, 57.8 \%)$. ${ }^{1} \mathrm{H}$ NMR $\left(300 \mathrm{MHz}, \mathrm{d}_{3^{-}}\right.$ acetonitrile): $\delta=7.37(\mathrm{~m}, 4 \mathrm{H}), \delta=7.20-7.33(\mathrm{~m}, 12 \mathrm{H}), \delta=7.04(\mathrm{~m}, 2 \mathrm{H}), \delta=7.00(\mathrm{~m}$, $6 \mathrm{H}), \delta=6.85(\mathrm{~m}, 3 \mathrm{H}) \delta=3.07\left(\mathrm{~m}, 8 \mathrm{H}, \mathrm{NBu}_{4}\right), \delta=1.56\left(\mathrm{~m}, 8 \mathrm{H}, \mathrm{NBu}_{4}\right), \delta=1.33(\mathrm{~m}, 8 \mathrm{H}$, $\left.\mathrm{NBu}_{4}\right), \delta=0.96\left(\mathrm{~m}, 12 \mathrm{H}, \mathrm{NBu}_{4}\right) .{ }^{13} \mathrm{C} \mathrm{NMR}\left(75.397 \mathrm{MHz}, \mathrm{CH}_{3} \mathrm{CN}\right): \delta=162,139.30$, 136.52, 135.95, 133.49, 131.72, 128.69, 128.58, 126.01, 125.97, 122.23, 58.69, 23.79, 19.81, 13.36. ${ }^{31} \mathrm{P}$ NMR (121.475 MHz, $\mathrm{d}_{3}$-acetonitrile): $\delta=-3.23(\mathrm{~s}) .{ }^{11} \mathrm{~B}$ NMR (400 $\mathrm{MHz}, \mathrm{d}_{3}$-acetonitrile): $\delta=-11.08$. Anal. Calcd. for $\mathrm{C}_{52} \mathrm{H}_{65} \mathrm{BNP}: \mathrm{C}, 83.74 ; \mathrm{H}, 8.78 ; \mathrm{N}$, 1.88. Found: $\mathrm{C}, 83.52 ; \mathrm{H}, 8.01 ; \mathrm{N}, 2.32$.

[NBu $\left.\mathbf{N u}_{4}\right]\left[\mathbf{P h}_{3} \mathbf{B P}^{m-\mathbf{i P r} 2}\right]$ (5). $m$ - $\mathrm{BrC}_{6} \mathrm{H}_{4} \mathrm{P}^{\mathrm{i}} \mathrm{Pr}_{2}(\mathbf{2 c})(0.671 \mathrm{~g}, 2.46 \mathrm{mmol})$ was dissolved in 75 $\mathrm{mL}$ dry, degassed THF and stirring solution was cooled to $-90{ }^{\circ} \mathrm{C}$. To this solution was 
added tert-BuLi (1.50 $\mathrm{M}$ in pentane, $1.64 \mathrm{~mL}, 2.46 \mathrm{mmol})$ and yellow solution was stirred for 1 hour at $-90^{\circ} \mathrm{C}$. Triphenylborane $(0.595 \mathrm{~g}, 2.46 \mathrm{mmol})$ was added as a THF solution and the solution was allowed to stir and warm to room temperature. Volatiles were removed in vacuo and remaining solids were taken up in $\mathrm{CH}_{2} \mathrm{Cl}_{2}$. A solution of $\mathrm{NBu}_{4} \mathrm{Br}(0.793 \mathrm{~g}, 2.46 \mathrm{mmol})$ in $\mathrm{CH}_{2} \mathrm{Cl}_{2}$ was added. Solution was allowed to stir for 3 hours, then filtered through Celite. Solvent was removed in vacuo and solids were washed with copious amounts of petroleum ether and diethyl ether to afford $5(0.802 \mathrm{~g}$, $48 \%$ ). This borate ligand is more difficult to crystallize than the others described, and its combustion analysis proved consequently high in carbon and low in nitrogen (two attempts). Crystals suitable for an X-ray diffraction study were grown via vapor diffusion of $\mathrm{Et}_{2} \mathrm{O}$ into THF. ${ }^{1} \mathrm{H}$ NMR $\left(300 \mathrm{MHz}, \mathrm{d}_{3}\right.$-acetonitrile): $\delta=7.51(\mathrm{~m}, 3 \mathrm{H}), \delta=7.37(\mathrm{~m}$, $6 \mathrm{H}), \delta=7.01(\mathrm{~m}, 1 \mathrm{H}), \delta=6.94(\mathrm{~m}, 6 \mathrm{H}), \delta=6.81(\mathrm{~m}, 3 \mathrm{H}) \delta=3.43\left(\mathrm{~m}, 8 \mathrm{H}, \mathrm{NBu}_{4}\right), \delta=$ $1.95(\mathrm{~m}, 2 \mathrm{H}), \delta=1.82\left(\mathrm{~m}, 8 \mathrm{H}, \mathrm{NBu}_{4}\right), \delta=1.45(\mathrm{~m}, 8 \mathrm{H}), \delta=1.12(\mathrm{~m}, 6 \mathrm{H}), \delta=0.99(\mathrm{~m}$, $\left.12 \mathrm{H}, \mathrm{NBu}_{4}\right), \delta=0.84(\mathrm{~m}, 6 \mathrm{H}) .{ }^{13} \mathrm{C}$ NMR $(75.397 \mathrm{MHz}$, acetonitrile): $\delta=162,136.40$, 135.96, 133.70, 128.77, 126.45, 125.85, 125.46, 122.08, 58.70, 23.80, 22.85, 20.06, 19.81, 18.76, 13.33. ${ }^{31} \mathrm{P}$ NMR (121.475 MHz, d 3 -acetonitrile): $\delta=13.99$ (s). ${ }^{11} \mathrm{~B}$ NMR (400 MHz, d $\mathrm{d}_{3}$-acetonitrile): $\delta=-11.02$ (s). Anal. Calcd. for $\mathrm{C}_{46} \mathrm{H}_{69} \mathrm{BNP}: \mathrm{C}, 81.51 ; \mathrm{H}$, 10.26; N, 2.07. Found: C, 83.07; H, 11.26; N, 1.32 .

$\left[\mathbf{N B u}_{4}\right]\left[\mathbf{P h}_{3} \mathbf{B} \mathbf{P}^{p-\mathrm{Pr} 2}\right]$ (6). $p$ - $\mathrm{BrC}_{6} \mathrm{H}_{4} \mathrm{P}^{\mathrm{i}} \operatorname{Pr}_{2}$ (2d) (1.391 g, $\left.5.11 \mathrm{mmol}\right)$ was dissolved in 75 $\mathrm{mL}$ dry, degassed THF and stirring solution was cooled to $-90{ }^{\circ} \mathrm{C}$. To this solution was added tert-BuLi $(1.50 \mathrm{M}$ in pentane, $3.41 \mathrm{~mL}, 5.11 \mathrm{mmol})$, providing a yellow solution that was stirred for 1 hour at $-90^{\circ} \mathrm{C}$. Triphenylborane $(1.235 \mathrm{~g}, 5.11 \mathrm{mmol})$ was added as a THF solution and the solution was stirred and allowed to warm to room temperature. Volatiles were removed in vacuo and the remaining solids were taken up in $\mathrm{CH}_{2} \mathrm{Cl}_{2}$. A solution of $\left[\mathrm{NBu}_{4}\right][\mathrm{Br}](1.647 \mathrm{~g}, 5.11 \mathrm{mmol})$ in $\mathrm{CH}_{2} \mathrm{Cl}_{2}$ was then added. The resulting solution was then allowed to stir for 3 hours, followed by filtration through Celite. Solvent was removed in vacuo and the solids were washed with copious amounts of petroleum ether and ether to afford $6(2.895 \mathrm{~g}, 84 \%)$. ${ }^{1} \mathrm{H} \mathrm{NMR}\left(300 \mathrm{MHz}, \mathrm{d}_{3^{-}}\right.$ acetonitrile): $\delta=7.26(\mathrm{~m}, 8 \mathrm{H}), \delta=7.13(\mathrm{~m}, 2 \mathrm{H}), \delta=7.00(\mathrm{~m}, 6 \mathrm{H}), \delta=6.85(\mathrm{~m}, 3 \mathrm{H}) \delta=$ $3.07\left(\mathrm{~m}, 8 \mathrm{H}, \mathrm{NBu}_{4}\right), \delta=2.05(\mathrm{~m}, 2 \mathrm{H}), \delta=1.59\left(\mathrm{~m}, 8 \mathrm{H}, \mathrm{NBu}_{4}\right), \delta=1.34(\mathrm{~m}, 8 \mathrm{H}), \delta=1.03$ $(\mathrm{m}, 6 \mathrm{H}), \delta=0.96\left(\mathrm{~m}, 12 \mathrm{H}, \mathrm{NBu}_{4}\right), \delta=0.88(\mathrm{~m}, 6 \mathrm{H}) .{ }^{13} \mathrm{C} \mathrm{NMR}(75.397 \mathrm{MHz}$, acetonitrile): $\delta=162,135.98,135.69,132.36,126.45,125.89,122.16,58.70,23.80$, 22.90, 20.13, 19.81, 18.91, 13.33. ${ }^{31} \mathrm{P}$ NMR (121.475 MHz, $\mathrm{d}_{3}$-acetonitrile): $\delta=12.42$ (s). ${ }^{11} \mathrm{~B}$ NMR (400 MHz, $\mathrm{d}_{3}$-acetonitrile): -11.13 (s). Anal. Calcd. for $\mathrm{C}_{46} \mathrm{H}_{69} \mathrm{BNP}: \mathrm{C}$, 81.51; H, 10.26; N, 2.07. Found: C, 81.71; H, 10.58; N, 2.26.

$\left(\mathbf{P h}_{3} \mathbf{S i P}^{m-\mathbf{P h} 2}\right.$ ) (7). $m$ - $\mathrm{BrC}_{6} \mathrm{H}_{4} \mathrm{P}^{\mathrm{i}} \mathrm{Pr}_{2}$ (2a) (1.391 g, $\left.5.11 \mathrm{mmol}\right)$ was dissolved in $75 \mathrm{~mL}$ of dry, degassed $\mathrm{Et}_{2} \mathrm{O}$ and stirred at $-90^{\circ} \mathrm{C}$. To this solution was added tert-BuLi $(1.50 \mathrm{M}$ in pentane, $3.41 \mathrm{~mL}, 5.11 \mathrm{mmol}$ ) to form a yellow solution that was stirred for 1 hour at -90 ${ }^{\circ} \mathrm{C}$. Triphenylsilylchloride $(1.235 \mathrm{~g}, 5.11 \mathrm{mmol})$ was then added as an $\mathrm{Et}_{2} \mathrm{O}$ solution, and the reaction mixture was stirred while allowing it to warm to room temperature. The final solution was filtered through Celite to remove $\mathrm{LiCl}$, and the volatiles were removed from the filtrate in vacuo. The remaining oil was taken up in toluene and refluxed for 6 hours to precipitate the remaining $\mathrm{LiCl}$ salts. The supernatant was filtered through a pad of Celite and the volatiles were again removed to yield the product $7(70 \%)$. ${ }^{1} \mathrm{H}$ NMR 
(300 MHz, $\mathrm{d}_{6}$-benzene): $\delta=7.96(\mathrm{~m}, 1 \mathrm{H}), \delta=7.64-7.77(\mathrm{~m}, 2 \mathrm{H}), \delta=7.58(\mathrm{~m}, 6 \mathrm{H}), \delta=$ $7.49(\mathrm{~m}, 1 \mathrm{H}), \delta=7.35(\mathrm{~m}, 4 \mathrm{H}), \delta=7.20(\mathrm{~m}, 4 \mathrm{H}), \delta=7.11(\mathrm{~m}, 6 \mathrm{H}), \delta=7.04(\mathrm{~m}, 2 \mathrm{H}), \delta=$ $7.00(\mathrm{~m}, 3 \mathrm{H}) .{ }^{31} \mathrm{P}$ NMR $\left(121.475 \mathrm{MHz}, \mathrm{d}_{6}\right.$-benzene): $\delta=-7.29(\mathrm{~s}) . \mathrm{ES}^{-\mathrm{MS}^{+}}(\mathrm{m} / \mathrm{z})=$ 521.2

$\left(\mathbf{P h}_{3} \mathbf{S i P}^{p-\mathrm{Ph} 2}\right)$ (8). $m$ - $\mathrm{BrC}_{6} \mathrm{H}_{4} \mathrm{P}^{\mathrm{i}} \mathrm{Pr}_{2}(\mathbf{2 b})(1.391 \mathrm{~g}, 5.11 \mathrm{mmol})$ was dissolved in $75 \mathrm{~mL}$ of dry, degassed $\mathrm{Et}_{2} \mathrm{O}$ and stirred at $-90^{\circ} \mathrm{C}$. To this solution was added tert-BuLi $(1.50 \mathrm{M}$ in pentane, $3.41 \mathrm{~mL}, 5.11 \mathrm{mmol}$ ) to form a yellow solution that was stirred for 1 hour at -90 ${ }^{\circ} \mathrm{C}$. Triphenylsilylchloride $(1.235 \mathrm{~g}, 5.11 \mathrm{mmol})$ was then added as an $\mathrm{Et}_{2} \mathrm{O}$ solution, and the reaction mixture was then stirred while being allowed to warm to room temperature. The resulting suspension was filtered through Celite to remove $\mathrm{LiCl}$ and the volatiles were removed from the filtrate in vacuo. The remaining oil was then extracted into toluene and refluxed for 6 hours to further precipitate the remaining $\mathrm{LiCl}$ salts. The supernatant was filtered through a pad of Celite and the volatiles were again removed to afford product $8(68 \%)$. ${ }^{1} \mathrm{H}$ NMR $\left(300 \mathrm{MHz}, \mathrm{d}_{6}\right.$-benzene): $\delta=7.46(\mathrm{~m}, 8 \mathrm{H}), \delta=7.34$ $(\mathrm{m}, 4 \mathrm{H}), \delta=7.30(\mathrm{~m}, 6 \mathrm{H}), \delta=7.26(\mathrm{~m}, 6 \mathrm{H}), \delta=7.19(\mathrm{~m}, 3 \mathrm{H}) .{ }^{31} \mathrm{P}$ NMR $(121.475 \mathrm{MHz}$, $\mathrm{d}_{6}$-benzene): $\delta=-9.21(\mathrm{~s}) . \mathrm{ES}^{-\mathrm{MS}^{+}}(\mathrm{m} / \mathrm{z})=521.2$

$\left(\mathbf{P h}_{3} \mathbf{S i P}^{m \text {-iPr2 }}\right.$ ) (9). $m-\mathrm{BrC}_{6} \mathrm{H}_{4} \mathrm{P}^{\mathrm{i}} \mathrm{Pr}_{2}(\mathbf{2 c})(1.391 \mathrm{~g}, 5.11 \mathrm{mmol})$ was dissolved in $75 \mathrm{~mL}$ of dry, degassed $\mathrm{Et}_{2} \mathrm{O}$ and stirred at $-90^{\circ} \mathrm{C}$. To this solution was added tert-BuLi $(1.50 \mathrm{M}$ in pentane, $3.41 \mathrm{~mL}, 5.11 \mathrm{mmol}$ ) to form a yellow solution that was stirred for 1 hour at -90 ${ }^{\circ} \mathrm{C}$. Triphenylsilylchloride $(1.235 \mathrm{~g}, 5.11 \mathrm{mmol})$ was added as an $\mathrm{Et}_{2} \mathrm{O}$ solution, and the resulting suspension was allowed to warm to room temperature while stirring. The mixture was filtered through Celite to remove $\mathrm{LiCl}$ and the volatiles were then removed in vacuo from the filtrate. The remaining oil was taken up in toluene and refluxed for 6 hours to further precipitate the remaining $\mathrm{LiCl}$ salts. The solution was then filtered through a pad of Celite and the solvent was removed to afford product $9(51 \%) .{ }^{1} \mathrm{H}$ NMR (300 MHz, $\mathrm{d}_{6}$-benzene): $\delta=7.98(\mathrm{~m}, 1 \mathrm{H}), \delta=7.69(\mathrm{~m}, 6 \mathrm{H}), \delta=7.65(\mathrm{~m}, 1 \mathrm{H}), \delta=$ $7.57(\mathrm{~m}, 1 \mathrm{H}), \delta=7.17(\mathrm{~m}, 9 \mathrm{H}), \delta=7.10(\mathrm{~m}, 1 \mathrm{H}), \delta=1.88(\mathrm{~m}, 2 \mathrm{H}), \delta=0.99(\mathrm{~m}, 6 \mathrm{H}), \delta=$ $0.87(\mathrm{~m}, 6 \mathrm{H}) .{ }^{13} \mathrm{C}$ NMR $\left(75.397 \mathrm{MHz}\right.$, acetonitrile): ${ }^{31} \mathrm{P}$ NMR $\left(121.475 \mathrm{MHz}, \mathrm{d}_{6^{-}}\right.$ benzene): $\delta=8.41(\mathrm{~s}) . \operatorname{ES}^{-M^{+}}(\mathrm{m} / \mathrm{z})=453.1$

$\left(\mathbf{P h}_{3} \mathbf{S i P}^{p-\mathrm{iPr}}\right)$ (10). $m$ - $\mathrm{BrC}_{6} \mathrm{H}_{4} \mathrm{P}^{\mathrm{i}} \mathrm{Pr}_{2}(\mathbf{2 d})(1.391 \mathrm{~g}, 5.11 \mathrm{mmol})$ was dissolved in $75 \mathrm{~mL}$ of dry, degassed $\mathrm{Et}_{2} \mathrm{O}$ and stirred at $-90^{\circ} \mathrm{C}$. To this solution was added tert-BuLi $(1.50 \mathrm{M}$ in pentane, $3.41 \mathrm{~mL}, 5.11 \mathrm{mmol}$ ) to form a yellow solution that was stirred for 1 hour at -90 ${ }^{\circ} \mathrm{C}$. Triphenylsilylchloride $(1.235 \mathrm{~g}, 5.11 \mathrm{mmol})$ was added as an $\mathrm{Et}_{2} \mathrm{O}$ solution, and the mixture was stirred and warmed gradually to room temperature. The resulting suspension was filtered through Celite to remove $\mathrm{LiCl}$ and the volatiles were then removed from the filtrate in vacuo. The remaining oil was extracted into toluene and refluxed for 6 hours to further precipitate the remaining $\mathrm{LiCl}$ salts. The solution was then filtered through a pad of Celite and the filtrate dried in vacuo to provide product 10 (56 \%). ${ }^{1} \mathrm{H}$ NMR (300 MHz, $\mathrm{d}_{6}$-benzene): $\delta=7.71(\mathrm{~m}, 2 \mathrm{H}), \delta=7.67(\mathrm{~m}, 6 \mathrm{H}), \delta=7.47(\mathrm{~m}$, $2 \mathrm{H}), \delta=7.16(\mathrm{~m}, 6 \mathrm{H}), \delta=7.11(\mathrm{~m}, 3 \mathrm{H}), \delta=1.95(\mathrm{~m}, 2 \mathrm{H}), \delta=1.02(\mathrm{~m}, 6 \mathrm{H}), \delta=0.90(\mathrm{~m}$, $6 \mathrm{H}) .{ }^{31} \mathrm{P}$ NMR (121.475 MHz, d $\mathrm{d}_{6}$-benzene): $\delta=11.36(\mathrm{~s}) . \mathrm{ES}^{-M^{+}}(\mathrm{m} / \mathrm{z})=453.1$ 
$\left.\{(\mathbf{N B D}) \mathbf{R h C l}]\left[\mathbf{P h}_{3} \mathbf{B P}^{m-\mathrm{iPr} 2}\right]\right\}\left\{\mathbf{N B u}_{4}\right\}$ (11). Solid [(NBD)RhCl $]_{2}(0.0144 \mathrm{~g}, 0.0224 \mathrm{mmol})$ was dissolved in THF. To this solution was added $6(0.0302 \mathrm{~g}, 0.0461 \mathrm{mmol})$ as a THF solution. The reaction solution immediately changed from yellow to orange. Volatiles were removed in vacuo to yield product that was spectroscopically pure (yield $\sim$ quantitative). ${ }^{1} \mathrm{H}$ NMR (300 MHz, $\mathrm{d}_{6}$-acetone): $\delta=7.33\left(\mathrm{~m}, 8 \mathrm{H}, o-\mathrm{BPh}_{3}\right), \delta=7.04(\mathrm{~m}$, $2 \mathrm{H}), \delta=6.96\left(\mathrm{~m}, 6 \mathrm{H}, m-\mathrm{BPh}_{3}\right), \delta=6.82\left(\mathrm{~m}, 3 \mathrm{H}, p-\mathrm{BPh}_{3}\right), \delta=3.62(\mathrm{~m}, 4 \mathrm{H}, \mathrm{NBD}), \delta=$ $3.52(\mathrm{br}, 2 \mathrm{H}, \mathrm{NBD}), \delta=3.42\left(\mathrm{~m}, 8 \mathrm{H}, \mathrm{NBu}_{4}\right), \delta=2.43\left(\mathrm{~m}, 2 \mathrm{H},{ }^{\mathrm{i}} \mathrm{Pr}\right), \delta=1.82(\mathrm{~m}, 8 \mathrm{H}$, $\left.\mathrm{NBu}_{4}\right), \delta=1.44\left(\mathrm{~m}, 8 \mathrm{H}, \mathrm{NBu}_{4}\right), \delta=1.22\left(\mathrm{~m}, 6 \mathrm{H},{ }^{\mathrm{i}} \mathrm{Pr}\right), \delta=1.08\left(\mathrm{~m}, 6 \mathrm{H},{ }^{\mathrm{i}} \mathrm{Pr}\right), \delta=0.99(\mathrm{~m}$, $\left.12 \mathrm{H}, \mathrm{NBu}_{4}\right), \delta=0.85$ (m, 2H, NBD). ${ }^{13} \mathrm{C} \mathrm{NMR}(75.397 \mathrm{MHz}$, acetonitrile): $\delta=163.6$, $138.25,136.07,135.72,130.89,128.60,126.06,122.35,67.78,58.86,51.63,50.98$, 50.00, 23.91, 23.02, 19.11, 19.94, 18.91, 13.45. ${ }^{31} \mathrm{P}$ NMR (121.475 MHz, $\mathrm{d}_{6}$-acetone): $44.88\left(\mathrm{~d},{ }^{1} J_{\mathrm{Rh}-\mathrm{P}}=166.4 \mathrm{~Hz}\right)$. Anal. Calcd. for $\mathrm{C}_{53} \mathrm{H}_{77} \mathrm{BClNPRh}: \mathrm{C}, 70.08 ; \mathrm{H}, 8.54 ; \mathrm{N}$, 1.54. Found: C, 69.76; H, 8.49; N, 1.72. Note: A crystallographic study was performed on crystals of $\mathbf{1 1}$ generated from a separate experiment that showed significant bromide incorporation at the halide site. The batch of ligand $\mathbf{6}$ used in that preparation had not been thoroughly freed of $\left[\mathrm{NBu}_{4}\right][\mathrm{Br}]$.

$\{\mathbf{A S N}\}_{2}\left\{\left[\mathbf{P h}_{3} \mathbf{B P}^{m-P h}\right]_{2} \mathbf{P t M e}_{2}\right\}$ (12). Solid (COD)PtMe 2 (0.0276 g, $\left.0.083 \mathrm{mmol}\right)$ and $\mathbf{3}$ $(0.1024 \mathrm{~g}, 0.169 \mathrm{mmol})$ were dissolved and stirred in THF at room temperature for one hour. White precipitate was collected on a fine frit and washed first with petroleum ether $(2 \times 2 \mathrm{~mL})$ and then with copious amounts of $\mathrm{Et}_{2} \mathrm{O}$. Thorough drying provided the desired white product 12 (isolated yield $=80 \%$ ). Crystals suitable for an X-ray diffraction study were grown from a crude product sample by vapor diffusion of $\mathrm{Et}_{2} \mathrm{O}$ into acetonitrile. ${ }^{1} \mathrm{H}$ NMR (300 MHz, d $\mathrm{d}_{6}$-acetone): $\delta=8.03(\mathrm{~m}, 1 \mathrm{H}), \delta=7.66(\mathrm{~m}, 1 \mathrm{H}), \delta=7.38(\mathrm{~m}, 1 \mathrm{H}), \delta=$ $7.30\left(\mathrm{~m}, 6 \mathrm{H}, o-\mathrm{BPh}_{3}\right), \delta=7.22(\mathrm{~m}, 1 \mathrm{H}), \delta=7.15\left(\mathrm{~m}, 4 \mathrm{H}, \mathrm{PPh}_{2}\right), \delta=7.00\left(\mathrm{~m}, 4 \mathrm{H}, \mathrm{PPh}_{2}\right)$, $\delta=6.86-6.96\left(\mathrm{~m}, 8 \mathrm{H}, \mathrm{PPh}_{2}, m-\mathrm{BPh}_{3}\right), \delta=6.80\left(\mathrm{~m}, 3 \mathrm{H}, p-\mathrm{BPh}_{3}\right), \delta=3.48(\mathrm{~m}, 16 \mathrm{H}, \mathrm{ASN})$, $\delta=2.09(\mathrm{~m}, 16 \mathrm{H}, \mathrm{ASN}), \delta=0.32\left(\mathrm{~m}, 6 \mathrm{H}, \mathrm{Pt}-\mathrm{Me},{ }^{2} J_{\mathrm{Pt}-\mathrm{H}}=72.0 \mathrm{~Hz}\right) .{ }^{13} \mathrm{C} \mathrm{NMR}(75.396$ $\mathrm{MHz}$, acetonitrile): $\delta=163,138.8,136.94,134.64,133.4,129.28,128.32,127.05$, 123.19, 65.10, 23.30, -19.5. ${ }^{31} \mathrm{P}$ NMR $\left(121.475 \mathrm{MHz}, \mathrm{d}_{6}\right.$-acetone): $28.63\left(\mathrm{~s},{ }^{1} J_{\mathrm{Pt}-\mathrm{P}}=1947\right.$ $\mathrm{Hz}$ ). Anal. Calcd. for $\mathrm{C}_{90} \mathrm{H}_{96} \mathrm{~B}_{2} \mathrm{~N}_{2} \mathrm{P} 2 \mathrm{Pt}: \mathrm{C}, 72.82 ; \mathrm{H}, 6.52 ; \mathrm{N}, 1.89$. Found: $\mathrm{C}, 72.45 ; \mathrm{H}$, $6.75 ; \mathrm{N}, 2.20$.

$\left\{\mathbf{N B u}_{4}\right\}_{2}\left\{\left[\mathbf{P h}_{3} \mathbf{B P}^{p-\mathrm{Ph}^{2}}\right]_{2} \mathbf{P t M e}_{2}\right\}$ (13). Solid (COD)PtMe 2 (0.0756 g, $\left.0.226 \mathrm{mmol}\right)$ and $\mathbf{4}$ $(0.3372 \mathrm{~g}, 0.453 \mathrm{mmol})$ were stirred in THF at room temperature for one hour. Volatiles were then removed in vacuo and the remaining solids were washed first with petroleum ether $(2 \times 2 \mathrm{~mL})$ and then with a copious amount of $\mathrm{Et}_{2} \mathrm{O}$ to provide, after drying, the desired product 13 (yield $\sim$ quantitative). The product was readily recrystallized from THF/Et ${ }_{2} \mathrm{O}$. ${ }^{1} \mathrm{H}$ NMR (300 MHz, $\mathrm{d}_{6}$-acetone): $\delta=7.43(\mathrm{~m}, 2 \mathrm{H}), \delta=7.38\left(\mathrm{~m}, 4 \mathrm{H}, \mathrm{PPh}_{2}\right), \delta$ $=7.36\left(\mathrm{~m}, 6 \mathrm{H}, o-\mathrm{BPh}_{3}\right), \delta=7.28\left(\mathrm{~m}, 4 \mathrm{H}, \mathrm{PPh}_{2}\right), \delta=7.08(\mathrm{~m}, 2 \mathrm{H}), \delta=6.94-7.01(\mathrm{~m}, 8 \mathrm{H}$, $\left.\mathrm{PPh}_{2}, m-\mathrm{BPh}_{3}\right), \delta=6.81\left(\mathrm{~m}, 3 \mathrm{H}, p-\mathrm{BPh}_{3}\right), \delta=3.38\left(\mathrm{~m}, 16 \mathrm{H}, \mathrm{NBu}_{4}\right), \delta=1.78(\mathrm{~m}, 16 \mathrm{H}$, $\left.\mathrm{NBu}_{4}\right), \delta=1.40\left(\mathrm{~m}, 16 \mathrm{H}, \mathrm{NBu}_{4}\right), \delta=0.96\left(\mathrm{~m}, 24 \mathrm{H}, \mathrm{NBu}_{4}\right), \delta=0.33\left(\mathrm{~m}, 6 \mathrm{H}, \mathrm{Pt}-\mathrm{Me},{ }^{2} J_{\mathrm{Pt}-\mathrm{H}}\right.$ $=69.5 \mathrm{~Hz}) \cdot{ }^{13} \mathrm{C}$ NMR $(75.397 \mathrm{MHz}$, acetonitrile): $\delta=164,136.30,135.68,134.32$, $133.66,129.08,128.91,127.60,126.20,122.50,122.23,58.87,23.87,19.91,13.44 .{ }^{31} \mathrm{P}$ NMR (121.475 MHz, d ${ }_{6}$-acetone): 27.47 (s, $\left.{ }^{1} J_{\mathrm{Pt}-\mathrm{P}}=1935 \mathrm{~Hz}\right)$. Anal. Calcd. for $\mathrm{C}_{106} \mathrm{H}_{136} \mathrm{~B}_{2} \mathrm{~N}_{2} \mathrm{P} 2 \mathrm{Pt}: \mathrm{C}, 74.15 ; \mathrm{H}, 7.56 ; \mathrm{N}, 1.63$. Found: C, 73.77; H, 8.18; N, 1.90. 
$\left\{\mathbf{N E t}_{4}\right\}_{2}\left\{\left(\mathbf{P h}_{3} \mathbf{B P}^{m-\mathrm{iPr}}\right)_{2} \mathbf{P t M e}_{2}\right\}$ (14). (COD)PtMe $2(0.0840 \mathrm{~g}, 0.252 \mathrm{mmol})$ and $\mathbf{5}(0.1681$ $\mathrm{g}, 0.298 \mathrm{mmol}$ ) were stirred in THF at room temperature for one hour. The reaction volatiles were then removed in vacuo and the resulting solids were washed with petroleum ether $(2 \times 2 \mathrm{~mL})$ and then copious amounts of $\mathrm{Et}_{2} \mathrm{O}$ to yield, after drying, the desired product 14 (yield $\sim$ quantitative). The product was easily recrystallized from THF/Et ${ }_{2} \mathrm{O} .{ }^{1} \mathrm{H}$ NMR $\left(300 \mathrm{MHz}, \mathrm{d}_{6}\right.$-acetone): $\delta=7.51(\mathrm{~m}, 1 \mathrm{H}), \delta=7.44(\mathrm{~m}, 1 \mathrm{H}), \delta=$ $7.31\left(\mathrm{~m}, 6 \mathrm{H}, o-\mathrm{BPh}_{3}\right), \delta=7.23(\mathrm{~m}, 1 \mathrm{H}), \delta=7.14(\mathrm{~m}, 1 \mathrm{H}), \delta=7.02\left(\mathrm{~m}, 6 \mathrm{H}, m-\mathrm{BPh}_{3}\right), \delta=$ $\left.6.88\left(\mathrm{~m}, 3 \mathrm{H}, p-\mathrm{BPh}_{3}\right), \delta=3.05\left(\mathrm{~m}, 16 \mathrm{H}, \mathrm{NEt}_{4}\right),\right)^{2}, \delta=2.21\left(\mathrm{~m}, 2 \mathrm{H},{ }^{\mathrm{i}} \mathrm{Pr}\right), \delta=1.42(\mathrm{~m}, 24 \mathrm{H}$, $\left.\mathrm{NEt}_{4}\right), \delta=0.98\left(\mathrm{~m}, 6 \mathrm{H},{ }^{\mathrm{i}} \mathrm{Pr}\right), \delta=0.88\left(\mathrm{~m}, 6 \mathrm{H},{ }^{\mathrm{i}} \mathrm{Pr}\right), \delta=0.17\left(\mathrm{~m}, 6 \mathrm{H}, \mathrm{Pt}-\mathrm{Me},{ }^{2} J_{\mathrm{Pt}-\mathrm{H}}=67.5\right.$ $\mathrm{Hz}) .{ }^{13} \mathrm{C}$ NMR (75.396 MHz, acetonitrile): $\delta=165$ (ipso-B), 140.33, 137.17, 136.36, 133.65, 128.86, 126.48, 126.11, 123.48, 122.32, 52.61, 23.91, 20.70, 18.73, 7.27, -10.93.

${ }^{31} \mathrm{P}$ NMR $\left(121.475 \mathrm{MHz}, \mathrm{d}_{6}\right.$-acetone): $38.11\left(\mathrm{~s},{ }^{1} \mathrm{~J}_{\mathrm{Pt} \mathrm{P}}=1910 \mathrm{~Hz}\right)$. Anal. Calcd. For $\mathrm{C}_{78} \mathrm{H}_{112} \mathrm{~B}_{2} \mathrm{~N}_{2} \mathrm{P}_{2} \mathrm{Pt}$ : C, 69.07; H, 8.32; N, 2.07. Found: C, 69.29; H, 8.17; N, 1.36.

$\left[\mathbf{N B u}_{4}\right]_{2}\left[\left(\mathbf{P h}_{3} \mathbf{B P}^{p-\mathbf{P r}^{2}}\right)_{2} \mathbf{P t M e}_{2}\right]$ (15). (COD)PtMe 2 (0.0840 g, $\left.0.252 \mathrm{mmol}\right)$ and $\mathbf{6}(0.1681$ $\mathrm{g}, 0.298 \mathrm{mmol}$ ) were stirred in THF at room temperature for one hour. The reaction volatiles were then removed in vacuo and the resulting solids were washed with petroleum ether $(2 \times 2 \mathrm{~mL})$ and then copious amounts of $\mathrm{Et}_{2} \mathrm{O}$ to provide the desired white product (yield $\sim$ quantitative). Product was recrystallized from THF/Et $2 \mathrm{O} .{ }^{1} \mathrm{H}$ NMR (300 MHz, $\mathrm{d}_{6}$-acetone): $\delta=7.25-7.34(\mathrm{~m}, 12 \mathrm{H}), \delta=7.18-7.24(\mathrm{~m}, 4 \mathrm{H}), \delta=7.07$ $(\mathrm{m}, 4 \mathrm{H}), \delta=6.93(\mathrm{~m}, 12 \mathrm{H}), \delta=6.79(\mathrm{~m}, 6 \mathrm{H}), \delta=3.41\left(\mathrm{~m}, 16 \mathrm{H}, \mathrm{NBu}_{4}\right), \delta=2.20(\mathrm{~m}, 4 \mathrm{H}$, $\left.{ }^{\mathrm{i}} \mathrm{Pr}\right), \delta=1.78\left(\mathrm{~m}, 16 \mathrm{H}, \mathrm{NBu}_{4}\right), \delta=1.41\left(\mathrm{~m}, 16 \mathrm{H}, \mathrm{NBu}_{4}\right), \delta=1.14\left(\mathrm{~m}, 12 \mathrm{H},{ }^{\mathrm{i}} \mathrm{Pr}\right), \delta=1.04$ $\left(\mathrm{m}, 12 \mathrm{H},{ }^{\mathrm{i}} \mathrm{Pr}\right), \delta=0.97\left(\mathrm{~m}, 24 \mathrm{H}, \mathrm{NBu}_{4}\right), \delta=0.30\left(\mathrm{~m}, 6 \mathrm{H}, \mathrm{Pt}-\mathrm{Me},{ }^{2} \mathrm{~J}_{\mathrm{Pt}-\mathrm{H}}=66.0 \mathrm{~Hz}\right) .{ }^{13} \mathrm{C}$ NMR (75.396 MHz, acetonitrile): $\delta=164$ (ipso-B), 138.17, 136.05, 134.86, 131.13, $125.93,122.19,67.75,58.78,23.85,20.58,19.88,18.92,13.41,-9.8 .{ }^{31} \mathrm{P}$ NMR $(121.475$ MHz, d 6 -acetone): $31.53\left(\mathrm{~s},{ }_{1}^{1} J_{\mathrm{Pt}-\mathrm{P}}=1907 \mathrm{~Hz}\right.$ ). Anal. Calcd. For $\mathrm{C}_{94} \mathrm{H}_{144} \mathrm{~B}_{2} \mathrm{~N}_{2} \mathrm{P}_{2} \mathrm{Pt}$ : C, 71.42; H, 9.18; N, 1.77. Found: C, 71.29; H, 9.21; N, 2.25.

$\left(\mathbf{P h}_{3} \mathbf{S i P}^{p \text {-iPr2 }}\right)_{2} \mathbf{P t M e}_{2}$ (16). (COD)PtMe $2(0.0840 \mathrm{~g}, 0.252 \mathrm{mmol})$ was stirred in the presence of $10(0.1681 \mathrm{~g}, 0.298 \mathrm{mmol})$ in THF at room temperature for one hour. The reaction volatiles were then removed in vacuo and the remaining solids were washed with petroleum ether $(2 \times 2 \mathrm{~mL})$ and $\mathrm{Et}_{2} \mathrm{O}(2 \times 2 \mathrm{~mL})$ to provide the desired white product quantitatively. ${ }^{1} \mathrm{H}$ NMR $\left(300 \mathrm{MHz}, \mathrm{d}_{6}\right.$-acetone): $\delta=7.34-7.64(\mathrm{~m}, 38 \mathrm{H}), \delta=2.42(\mathrm{~m}$, $\left.4 \mathrm{H},{ }^{\mathrm{i}} \mathrm{Pr}\right), \delta=1.13\left(\mathrm{~m}, 12 \mathrm{H},{ }^{\mathrm{i}} \mathrm{Pr}\right), \delta=1.01\left(\mathrm{~m}, 12 \mathrm{H},{ }^{\mathrm{i}} \mathrm{Pr}\right), \delta=0.30\left(\mathrm{~m}, 6 \mathrm{H}, \mathrm{Pt}-\mathrm{Me},{ }^{2} J_{\mathrm{Pt}-\mathrm{H}}=\right.$ $63.0 \mathrm{~Hz}) .{ }^{31} \mathrm{P}$ NMR $\left(121.475 \mathrm{MHz}, \mathrm{d}_{6}\right.$-acetone): $36.94\left(\mathrm{~s},{ }^{1} J_{\mathrm{Pt} \mathrm{P}}=1883 \mathrm{~Hz}\right)$.

$\left(\mathbf{P h}_{3} \mathbf{S i P}^{p-{ }^{-P h}}\right)_{2} \mathbf{P t M e}_{2}$ (17). Prepared as for the case of 16. ${ }^{1} \mathrm{H}$ NMR $\left(300 \mathrm{MHz}, \mathrm{d}_{6}\right.$ acetone): $\delta=7.45(\mathrm{~m}, 12 \mathrm{H}), \delta=7.16-7.45(\mathrm{~m}, 46 \mathrm{H}), \delta=0.39\left(\mathrm{~m}, 6 \mathrm{H}, \mathrm{Pt}-\mathrm{Me},{ }^{2} J_{\mathrm{Pt}-\mathrm{H}}=\right.$ $60.0 \mathrm{~Hz}) .{ }^{31} \mathrm{P}$ NMR $\left(121.475 \mathrm{MHz}, \mathrm{d}_{6}\right.$-acetone): $30.45\left(\mathrm{~s},{ }^{1} J_{\mathrm{Pt}-\mathrm{P}}=1900 \mathrm{~Hz}\right)$.

[cis-( $\left.\left.\mathbf{P h}_{3} \mathbf{S i P}^{p-P h}\right)_{2} \mathbf{P t M e}(\mathbf{s o l v})\right]\left[\mathbf{M e}\left(\mathbf{B}\left(\mathbf{C}_{6} \mathbf{F}_{5}\right)_{3}\right]\right.$ (18). Complex 17 (0.0195 g, 0.0154 mmol) was stirred in a THF solution containing $\mathrm{B}\left(\mathrm{C}_{6} \mathrm{~F}_{5}\right)_{3}(0.0079 \mathrm{~g}, 0.0154 \mathrm{mmol})$ for 12 hours. The ${ }^{31} \mathrm{P}$ NMR spectrum verified clean generation of a single cis mono-solvento, mono-methyl species (18). ${ }^{1} \mathrm{H}$ NMR $\left(300 \mathrm{MHz}, \mathrm{d}_{6}\right.$-acetone): $\delta=6.98-8.02(\mathrm{~m}, 58 \mathrm{H}), \delta=$ 0.52 (br s, $3 \mathrm{H}, \mathrm{Me}\left(\mathrm{B}\left(\mathrm{C}_{6} \mathrm{~F}_{5}\right)_{3}\right), \delta=0.61\left(\mathrm{~m}, 3 \mathrm{H}, \mathrm{Pt}-\mathrm{Me},{ }^{2} J_{\mathrm{Pt}-\mathrm{H}}=54.0 \mathrm{~Hz}\right) .{ }^{31} \mathrm{P}$ NMR (121.475 MHz, d 6 -acetone): $24.4\left(\mathrm{~d},{ }^{1} J_{\mathrm{Pt}-\mathrm{P}}=1942 \mathrm{~Hz},{ }^{2} J_{\mathrm{P}-\mathrm{P}}=396 \mathrm{~Hz}\right)$. 
$\left\{\mathbf{N B u}_{4}\right\}\left\{\mathbf{M o}(\mathbf{C O})_{5}\left[\mathbf{P h}_{3} \mathbf{B P}^{m-\mathrm{iPr} 2}\right]\right\}$ (19). Solid Mo $\left(\mathrm{CO}_{6}\right)(0.0078 \mathrm{~g}, 0.030 \mathrm{mmol})$ and solid $5(0.0204 \mathrm{~g}, 0.0301 \mathrm{mmol})$ were combined in THF and refluxed for 12 hours to provide a yellow solution. Volatiles were then removed in vacuo. The solid product was recrystallized from THF/Et ${ }_{2} \mathrm{O}$ to provide 19. ${ }^{1} \mathrm{H}$ NMR $\left(300 \mathrm{MHz}, \mathrm{d}_{6}\right.$-acetonitrile): $\delta=$ $7.42(\mathrm{~m}, 3 \mathrm{H}), \delta=7.34(\mathrm{~m}, 6 \mathrm{H}), \delta=7.11(\mathrm{~m}, 1 \mathrm{H}), \delta=6.95(\mathrm{~m}, 6 \mathrm{H}), \delta=6.80(\mathrm{~m}, 3 \mathrm{H}) \delta=$ $3.43\left(\mathrm{~m}, 8 \mathrm{H}, \mathrm{NBu}_{4}\right), \delta=2.32(\mathrm{~m}, 2 \mathrm{H}), \delta=1.81\left(\mathrm{~m}, 8 \mathrm{H}, \mathrm{NBu}_{4}\right), \delta=1.42(\mathrm{~m}, 8 \mathrm{H}), \delta=1.07$ $\left(\mathrm{m}, 6 \mathrm{H},{ }^{\mathrm{i}} \mathrm{Pr}\right), \delta=0.97\left(\mathrm{~m}, 12 \mathrm{H}, \mathrm{NBu}_{4}\right), \delta=0.91\left(\mathrm{~m}, 6 \mathrm{H},{ }^{\mathrm{i}} \mathrm{Pr}\right) .{ }^{31} \mathrm{P} \mathrm{NMR}(121.475 \mathrm{MHz}$, $\mathrm{d}_{6}$-acetone): $\delta=52.33$ (s). IR: $\left(\mathrm{KBr} / \mathrm{CH}_{2} \mathrm{Cl}_{2}\right): 2065,1925 \mathrm{~cm}^{-1} . \mathrm{ES} \mathrm{MS}^{-}(\mathrm{m} / \mathrm{z})=671$, 643,615 .

$\left.\left[\mathbf{N B u}_{4}\right]\left[\mathbf{M o}(\mathbf{C O})_{5}\left(\mathbf{P h}_{3} \mathbf{S i P}^{m-\mathrm{iPr} 2}\right)\right] \mathbf{( 2 0}\right)$. Solid $\mathrm{Mo}\left(\mathrm{CO}_{6}\right)(0.0078 \mathrm{~g}, 0.030 \mathrm{mmol})$ and solid $9(0.0204 \mathrm{~g}, 0.0301 \mathrm{mmol})$ were combined in THF and refluxed for 12 hours to provide a yellow solution. The reactions volatiles were removed in vacuo and the remaining solid product was recrystallized from THF/Et ${ }_{2} \mathrm{O} .{ }^{1} \mathrm{H}$ NMR $\left(300 \mathrm{MHz}, \mathrm{d}_{6}\right.$-benzene): $\delta=7.68$ $(\mathrm{m}, 1 \mathrm{H}), \delta=7.59(\mathrm{~m}, 6 \mathrm{H}), \delta=7.47(\mathrm{~m}, 1 \mathrm{H}), \delta=7.29(\mathrm{~m}, 1 \mathrm{H}), \delta=7.16(\mathrm{~m}, 9 \mathrm{H}), \delta=7.10$ $(\mathrm{m}, 1 \mathrm{H}), \delta=2.03(\mathrm{~m}, 2 \mathrm{H}), \delta=1.04(\mathrm{~m}, 6 \mathrm{H}), \delta=0.89(\mathrm{~m}, 6 \mathrm{H}) .{ }^{31} \mathrm{P}$ NMR $(121.475 \mathrm{MHz}$, $\mathrm{d}_{6}$-benzene): $\delta=51.86$ (s). IR: $\left(\mathrm{KBr} / \mathrm{CH}_{2} \mathrm{Cl}_{2}\right): 2070,1942 \mathrm{~cm}^{-1}$.

General Procedure for Suzuki coupling reactions. $\mathrm{Pd}_{2}(\mathrm{dba})_{3}(0.0046 \mathrm{~g}, 1.5 \mathrm{~mol} \%), 5$ (0.0135 g, $6.0 \mathrm{~mol} \%), \mathrm{PhB}(\mathrm{OH})_{2}(0.0446 \mathrm{~g}, 0.367 \mathrm{mmol})$, and $\mathrm{Cs}_{2} \mathrm{CO}_{3}(0.2606 \mathrm{~g}, 0.800$ mmol) were combined under air in a $5 \mathrm{~mL}$ screw-cap vial equipped with a stir bar. The vial was then sealed with a septum and flushed thoroughly with nitrogen, after which time one equivalent of the appropriate aryl halide was added as a THF solution (dry, 1.5 $\mathrm{mL}$ ). While under nitrogen, the septum was quickly replaced with a Teflon-lined cap. The reaction mixture was then refluxed with stirring for 24 hours. The mixture was then diluted with copious amounts of ether and filtered through a silica plug. Volatiles were removed in vacuo, and the product was purified by flash chromatography.

4-chlorobiphenyl. Aryl halide: 1,4-dichlorobenzene (0.0489 g, $0.333 \mathrm{mmol})$. Product was isolated via column chromatography (hexanes). ${ }^{1} \mathrm{H}$ NMR $\left(300 \mathrm{MHz}, \mathrm{CDCl}_{3}\right): \delta=$ 7.37-7.58 (m, 9H).

4-methylbiphenyl. Aryl halide: 4-chlorotoluene $(39.4 \mu \mathrm{L}, 0.333 \mathrm{mmol})$. Product was isolated via column chromatography (hexanes). ${ }^{1} \mathrm{H}\left(\mathrm{NMR}\left(300 \mathrm{MHz}, \mathrm{CDCl}_{3}\right): \delta=7.57\right.$ $(\mathrm{m}, 2 \mathrm{H}), \delta=7.49(\mathrm{~m}, 2 \mathrm{H}), \delta=7.42(\mathrm{~m}, 2 \mathrm{H}), \delta=7.32(\mathrm{~m}, 1 \mathrm{H}), \delta=7.35(\mathrm{~m}, 2 \mathrm{H}), \delta=2.39$ $(\mathrm{s}, 3 \mathrm{H})$.

4-phenylacetophenone. Aryl halide: 4-chloroacetophenone (43.2 $\mu \mathrm{L}, 0.333 \mathrm{mmol})$. Product was isolated via column chromatography (1\% EtOAc/hexanes). ${ }^{1} \mathrm{H}$ NMR (300 $\left.\mathrm{MHz}, \mathrm{CDCl}_{3}\right): \delta=8.03(\mathrm{~m}, 2 \mathrm{H}), \delta=7.69(\mathrm{~m}, 2 \mathrm{H}), \delta=7.63(\mathrm{~m}, 2 \mathrm{H}), \delta=7.47(\mathrm{~m}, 2 \mathrm{H})$, $7.40(\mathrm{~m}, 1 \mathrm{H}), \delta=2.64(\mathrm{~s}, 3 \mathrm{H})$. 
Note on Suzuki coupling reactions: A point of concern pertains to whether the tetraarylborate unit of ligand $\mathbf{5}$ is transferred during the cross-coupling reactions. While we cannot rule-out this possibility altogether, we note that (i) tolylboronic acids were also screened and found to give comparable yields of cross-coupled products. Also, the yield of cross-coupled product far exceeds the molar ratio of the (phosphino)borate ligand, which was used in catalytic quantity. Thus, aryl transfer from the ligand is not critical to cross-coupling of the chloride substrates shown in Table 1 in the main text.

Additional Suzuki Coupling Data using o-tolylboronic acid:

The following data were obtained as non-optimized yields by crude GC-MS analysis. 


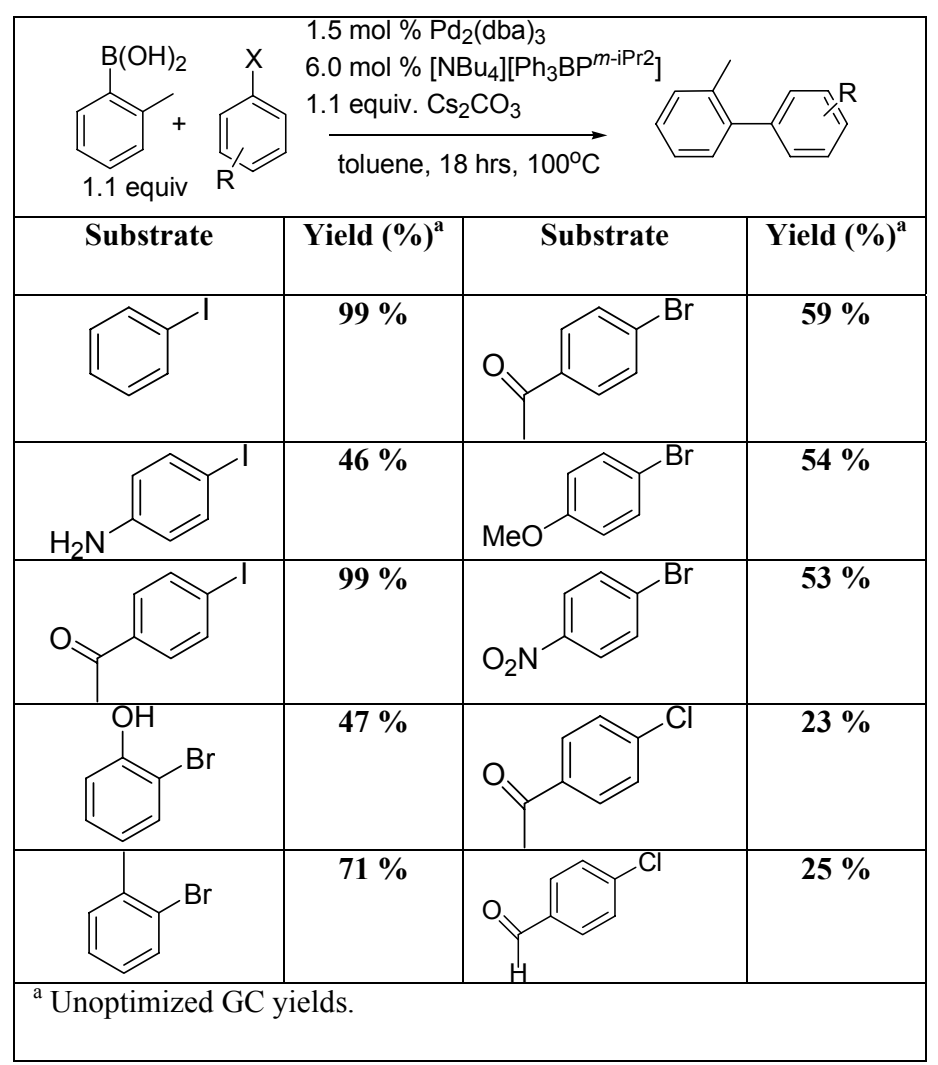

Figure 1: Fully labeled drawing of $\left\{\left[\mathrm{NEt}_{4}\right]\left[\mathrm{Ph}_{3} \mathrm{BP}^{m-\mathrm{Ph} 2}\right]\right\}_{2}$. Hydrogens have been omitted for clarity. One of the two cations in the unit cell was found to be disordered over two positions, contributing to the high GOF value for this structure. Only one of these positions is modeled in the following representation. 

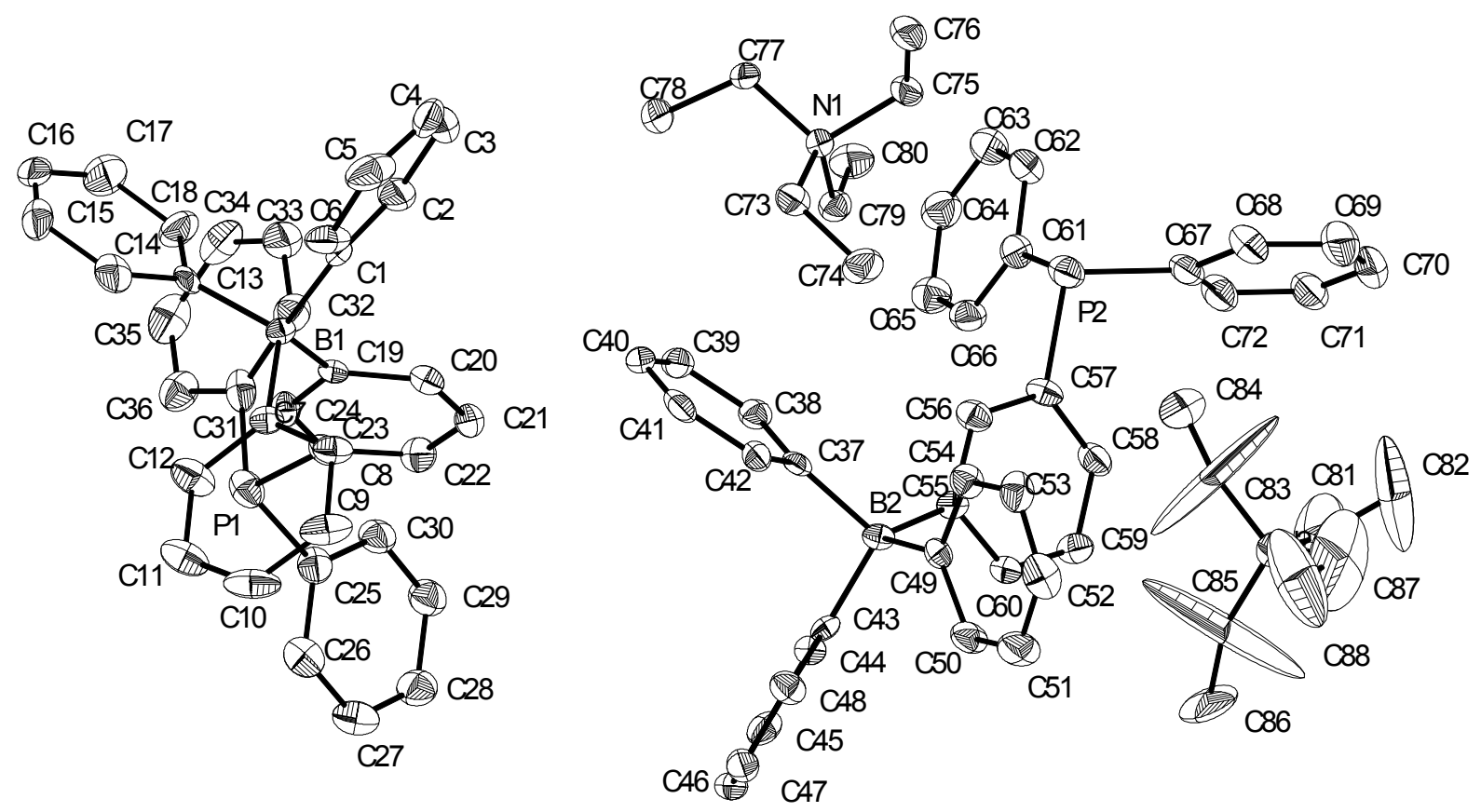

Figure 2: Fully labeled drawing of $\left\{\left[\mathrm{NEt}_{4}\right]\left[\mathrm{Ph}_{3} \mathrm{BP}^{m-\mathrm{iPr} 2}\right]\right\}_{2}$. Hydrogens have been omitted for clarity. 


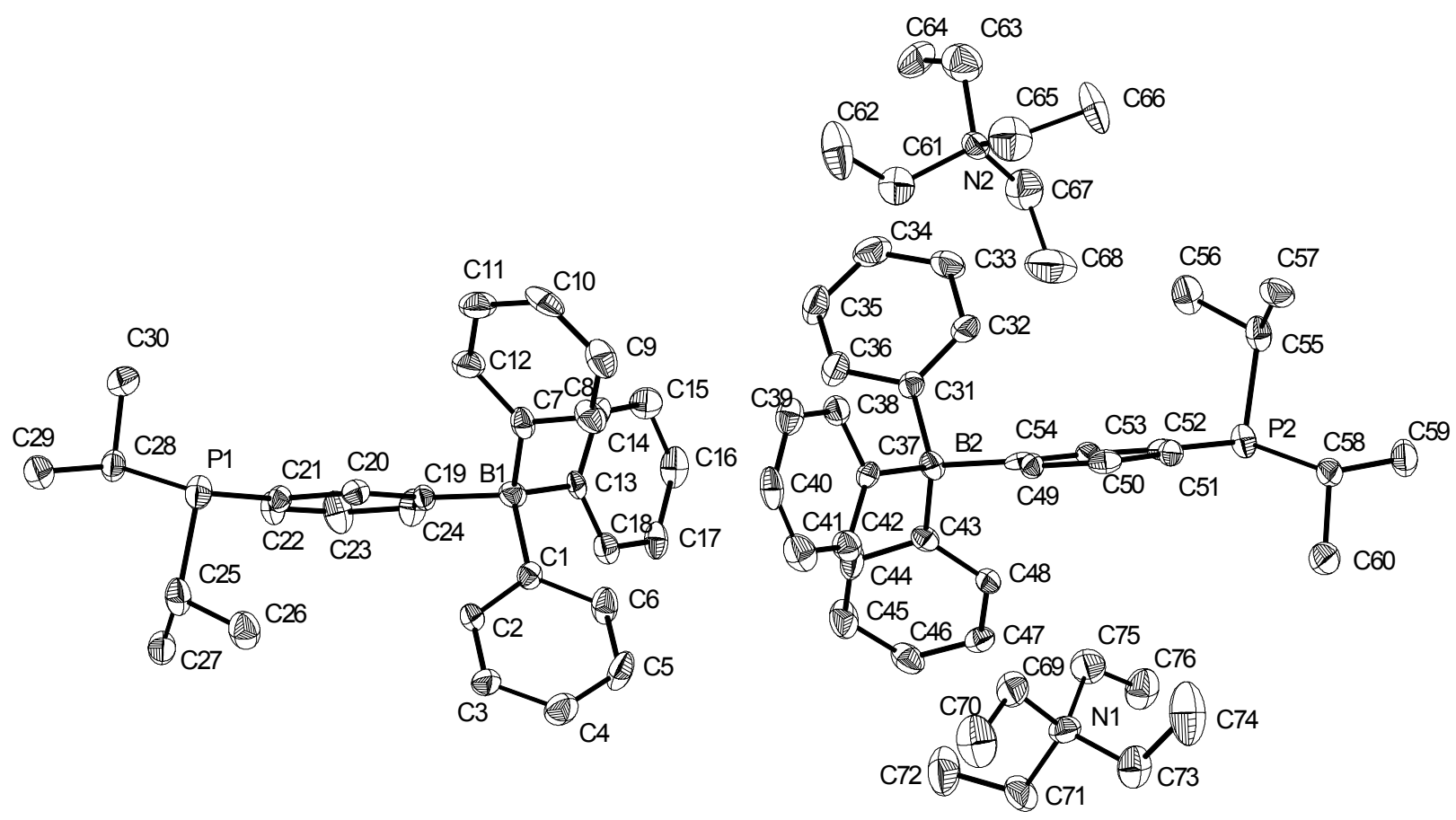

Figure 3: Fully labeled drawing of $\left\{\mathrm{NBu}_{4}\right\}\left\{\left[\mathrm{Ph}_{3} \mathrm{BP}^{p-\mathrm{PPr} 2}\right] \mathrm{RhX}(\mathrm{NBD})\right\}$ (X modeled as $\mathrm{Br}$ $(60 \%), \mathrm{Cl}(40 \%))$. Hydrogens have been omitted for clarity. Bromide contamination in this crystal likely resulted from the presence of $\left[\mathrm{NBu}_{4}\right][\mathrm{Br}]$ in the batch of ligand used to prepare the rhodium sample. This crystal structure was obtained prior to optimization of the ligand purifaction procedure, and serves to establish the proposed connectivity. Pure 
$\left\{\mathrm{NBu}_{4}\right\}\left\{\left[\mathrm{Ph}_{3} \mathrm{BP}^{p-\mathrm{iPr} 2}\right] \mathrm{RhCl}(\mathrm{NBD})\right\}$ can be obtained by the procedure outlined in the experimental section above using $\left[\mathrm{NBu}_{4}\right]\left[\mathrm{Ph}_{3} \mathrm{BP}^{p \text {-iPr2}}\right]$ free of $\left[\mathrm{NBu}_{4}\right][\mathrm{Br}]$ contaminant.

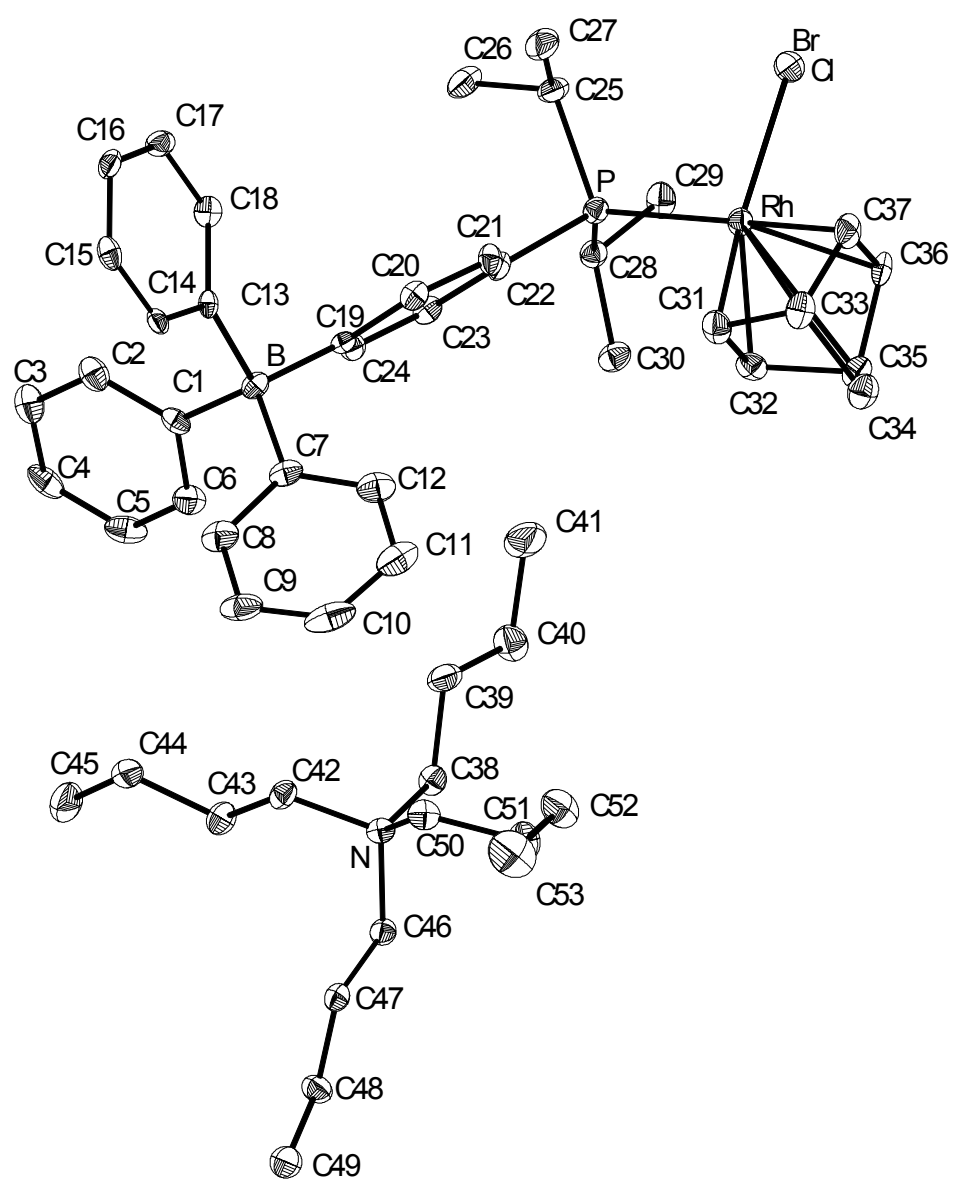

Figure 4: Fully labeled drawing of $\{\mathrm{ASN}\}_{2}\left\{\left[\mathrm{Ph}_{3} \mathrm{BP}^{m-\mathrm{Ph} 2}\right]_{2} \mathrm{PtMe}_{2}\right\}$. Hydrogens have been omitted for clarity. This crystal co-crystallized with one equiv of an [ASN][Br] salt, and one solvent molecule $\left(\mathrm{Et}_{2} \mathrm{O}\right)$. These have been omitted for clarity. The [ASN][Br] 
contamination arises from its presence in the sample of $[\mathrm{ASN}]\left[\mathrm{Ph}_{3} \mathrm{BP}^{m-\mathrm{Ph} 2}\right]$ used to prepare this sample. This sample that afforded this crystal structure was obtained prior to the optimization of the preparation of $[\mathrm{ASN}]\left[\mathrm{Ph}_{3} \mathrm{BP}^{m-\mathrm{Ph2}}\right]$, which frees the ligand from $[\mathrm{ASN}][\mathrm{Br}]$.

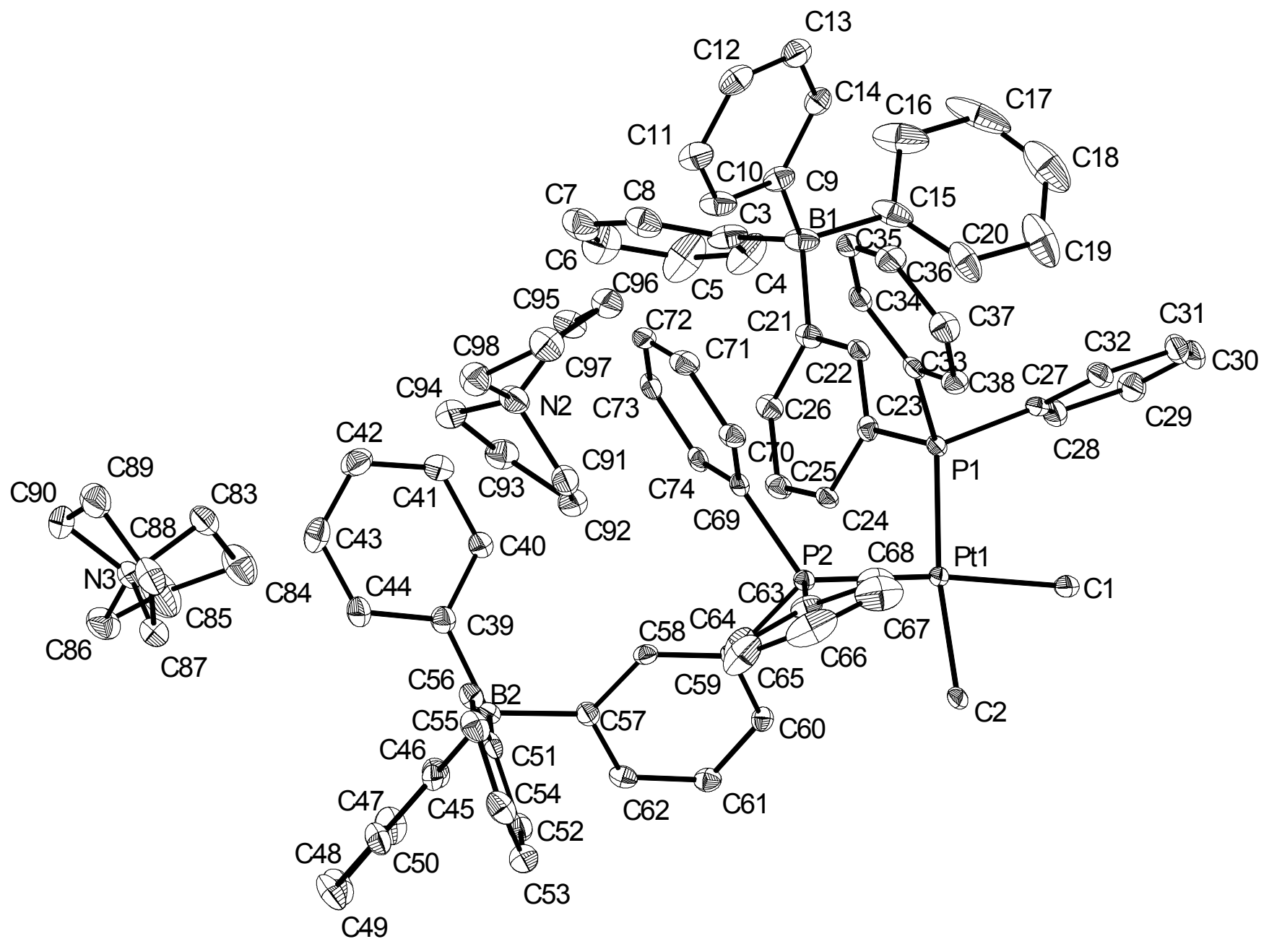


Table 1. Crystal data and structure refinement for $\left\{\left[\mathrm{NEt}_{4}\right]\left[\mathrm{Ph}_{3} \mathrm{BP}^{m-\mathrm{Ph}^{2}}\right]\right\}_{2}$

Empirical formula

Formula weight

Crystal Habit

Crystal Color

Crystal size

Type of diffractometer

Wavelength

Data collection temperature

Unit cell dimensions

Volume

Z

Crystal system

Space group

Density (calculated)

$\mathrm{F}(000)$

$\theta$ range for data collection

Completeness to $\theta=28.58^{\circ}$

Index ranges

Reflections collected

Independent reflections

Absorption coefficient

Absorption correction
$\mathrm{C}_{44} \mathrm{H}_{49} \mathrm{BNP}$

633.62

plate

colorless

$0.581 \times 0.24 \times 0.085 \mathrm{~mm}^{3}$

\section{Data Collection}

CCD area detector

$0.71073 \AA$

98(2) K
$\mathrm{a}=18.0519(13) \AA$
$\alpha=90^{\circ}$
$\mathrm{b}=10.4941(8) \AA$
$\mathrm{c}=38.060(3) \AA$
$\beta=90.4990(10)^{\circ}$
$\gamma=90^{\circ}$

7209.7(9) $\AA^{3}$

8

Monoclinic

$\mathrm{P} 2{ }_{1}$

$1.167 \mathrm{Mg} / \mathrm{m}^{3}$

2720

1.55 to $28.58^{\circ}$

$93.1 \%$

$-24 \leq \mathrm{h} \leq 23,-13 \leq \mathrm{k} \leq 14,-49 \leq 1 \leq 50$

122531

$17132[\mathrm{R}(\mathrm{int})=0.1101]$

$0.108 \mathrm{~mm}^{-1}$

None

\section{Structure solution and refinement}

Structure solution program

Primary solution method

Secondary solution method

Hydrogen placement

SHELXS-97 (Sheldrick (1990)

direct methods

Difference Fourier Map

calculated positions

Structure refinement program

SHELXL-97 (Sheldrick (1997) 
Refinement method

Data / restraints / parameters

Goodness-of-fit on $\mathrm{F}^{2}$

Final $\mathrm{R}$ indices $[\mathrm{I}>2 \operatorname{sigma}(\mathrm{I})]$

$\mathrm{R}$ indices (all data)

Largest diff. peak and hole
Full-matrix least-squares on $\mathrm{F}^{2}$

$17132 / 0 / 856$

2.159

$\mathrm{R} 1=0.0903, \mathrm{wR} 2=0.1242$

$\mathrm{R} 1=0.1742, \mathrm{wR} 2=0.1309$

1.538 and -1.535 e. $\AA^{-3}$

\section{Special Refinement Details:}

One of the cations in the unit cell is disordered. Attempts to model the disorder were unsuccessful. 
Table 2. Atomic coordinates $\left(\times 10^{4}\right)$ and equivalent isotropic displacement parameters $\left(\AA^{2} \times 10^{3}\right)$ for $\left\{\left[\mathrm{NEt}_{4}\right]\left[\mathrm{Ph}_{3} \mathrm{BP}^{m-\mathrm{Ph} 2}\right]\right\}_{2}$. U(eq) is defined as one third of the trace of the orthogonalized $\mathrm{Uij}^{\mathrm{ij}}$ tensor.

\begin{tabular}{|c|c|c|c|c|}
\hline & $\mathrm{x}$ & $y$ & $\mathrm{z}$ & $\mathrm{U}(\mathrm{eq})$ \\
\hline $\mathrm{B}(1)$ & 1098(2) & $8608(3)$ & $935(1)$ & $18(1)$ \\
\hline $\mathrm{P}(1)$ & 1611(1) & $12247(1)$ & 1886(1) & $30(1)$ \\
\hline$C(1)$ & $1144(2)$ & 7051(3) & $877(1)$ & $23(1)$ \\
\hline$C(2)$ & $1278(2)$ & 6206(3) & 1148(1) & $41(1)$ \\
\hline$C(3)$ & $1278(2)$ & 4853(4) & 1090(1) & $49(1)$ \\
\hline$C(4)$ & $1139(2)$ & 4418(4) & $756(1)$ & $49(1)$ \\
\hline$C(5)$ & $981(2)$ & $5205(4)$ & $484(1)$ & $49(1)$ \\
\hline $\mathrm{C}(6)$ & $995(2)$ & $6508(3)$ & $550(1)$ & $36(1)$ \\
\hline$C(7)$ & $1398(2)$ & $9370(3)$ & $587(1)$ & $20(1)$ \\
\hline$C(8)$ & 1982(2) & 8909(3) & $385(1)$ & $27(1)$ \\
\hline C(9) & $2290(2)$ & 9589(4) & 111(1) & $35(1)$ \\
\hline$C(10)$ & $2028(2)$ & $10781(4)$ & $31(1)$ & $43(1)$ \\
\hline$C(11)$ & 1451(2) & $11277(4)$ & $217(1)$ & $42(1)$ \\
\hline$C(12)$ & $1150(2)$ & $10577(3)$ & 492(1) & $36(1)$ \\
\hline$C(13)$ & $226(2)$ & $8857(3)$ & 1019(1) & $20(1)$ \\
\hline$C(14)$ & $-318(2)$ & $9025(3)$ & $758(1)$ & $33(1)$ \\
\hline$C(15)$ & $-1077(2)$ & 9079(3) & $833(1)$ & $38(1)$ \\
\hline$C(16)$ & $-1305(2)$ & 8973(3) & $1170(1)$ & $43(1)$ \\
\hline$C(17)$ & $-800(2)$ & 8807(4) & 1434(1) & $52(1)$ \\
\hline$C(18)$ & $-53(2)$ & $8760(3)$ & $1355(1)$ & $37(1)$ \\
\hline$C(19)$ & 1653(2) & $9127(3)$ & $1249(1)$ & 19(1) \\
\hline$C(20)$ & $2335(2)$ & 8574(3) & 1333(1) & $23(1)$ \\
\hline $\mathrm{C}(21)$ & 2819(2) & 9093(3) & 1581(1) & $26(1)$ \\
\hline$C(22)$ & $2630(2)$ & 10193(3) & $1752(1)$ & $27(1)$ \\
\hline$C(23)$ & 1963(2) & $10803(3)$ & $1676(1)$ & $23(1)$ \\
\hline$C(24)$ & 1501(2) & 10253(3) & 1424(1) & $23(1)$ \\
\hline$C(25)$ & 2431(2) & $12950(3)$ & 2098(1) & $29(1)$ \\
\hline$C(26)$ & $2769(2)$ & $13968(3)$ & 1930(1) & $33(1)$ \\
\hline$C(27)$ & $3404(2)$ & $14506(3)$ & $2070(1)$ & $38(1)$ \\
\hline$C(28)$ & 3696(2) & 14054(3) & $2382(1)$ & $36(1)$ \\
\hline$C(29)$ & $3362(2)$ & $13063(3)$ & 2553(1) & $35(1)$ \\
\hline $\mathrm{C}(30)$ & $2736(2)$ & $12507(3)$ & $2412(1)$ & $30(1)$ \\
\hline$C(31)$ & $1135(2)$ & $11585(3)$ & $2266(1)$ & $30(1)$ \\
\hline$C(32)$ & 1134(2) & 10302(3) & $2360(1)$ & $34(1)$ \\
\hline $\mathrm{C}(33)$ & 743(2) & 9858(4) & $2645(1)$ & $40(1)$ \\
\hline$C(34)$ & $342(2)$ & $10689(4)$ & $2852(1)$ & $43(1)$ \\
\hline$C(35)$ & $331(2)$ & $11968(4)$ & 2761(1) & $44(1)$ \\
\hline$C(36)$ & $718(2)$ & $12402(4)$ & 2474(1) & $36(1)$ \\
\hline $\mathrm{B}(2)$ & 6141(2) & $6265(3)$ & $959(1)$ & $17(1)$ \\
\hline $\mathrm{P}(2)$ & $6254(1)$ & $2867(1)$ & 2049(1) & $30(1)$ \\
\hline$C(37)$ & $5248(2)$ & $5948(3)$ & $910(1)$ & $18(1)$ \\
\hline$C(38)$ & 4744(2) & 6059(3) & 1186(1) & $24(1)$ \\
\hline$C(39)$ & $3972(2)$ & $5980(3)$ & $1133(1)$ & $27(1)$ \\
\hline$C(40)$ & $3694(2)$ & $5779(3)$ & 799(1) & $28(1)$ \\
\hline $\mathrm{C}(41)$ & $4168(2)$ & $5642(3)$ & $520(1)$ & $29(1)$ \\
\hline$C(42)$ & 4927(2) & $5720(3)$ & $577(1)$ & $23(1)$ \\
\hline
\end{tabular}




\begin{tabular}{|c|c|c|c|c|}
\hline C(43) & $6189(2)$ & 7822(3) & $926(1)$ & $18(1)$ \\
\hline $\mathrm{C}(44)$ & $6193(2)$ & $8638(3)$ & $1216(1)$ & $23(1)$ \\
\hline$C(45)$ & $6232(2)$ & 9962(3) & $1180(1)$ & $27(1)$ \\
\hline$C(46)$ & $6257(2)$ & $10508(3)$ & $851(1)$ & $29(1)$ \\
\hline $\mathrm{C}(47)$ & $6234(2)$ & 9737(3) & $560(1)$ & $31(1)$ \\
\hline $\mathrm{C}(48)$ & 6193(2) & 8432(3) & 601(1) & $27(1)$ \\
\hline$C(49)$ & $6617(2)$ & 5539(3) & $650(1)$ & $19(1)$ \\
\hline$C(50)$ & $7205(2)$ & 6095(3) & $470(1)$ & $30(1)$ \\
\hline $\mathrm{C}(51)$ & $7589(2)$ & 5458(4) & 211(1) & $39(1)$ \\
\hline$C(52)$ & $7415(2)$ & 4219(4) & $124(1)$ & $34(1)$ \\
\hline$C(53)$ & $6868(2)$ & 3619(3) & $310(1)$ & $31(1)$ \\
\hline$C(54)$ & $6483(2)$ & 4264(3) & $565(1)$ & $26(1)$ \\
\hline $\mathrm{C}(55)$ & 6513(2) & 5764(3) & 1328(1) & $18(1)$ \\
\hline$C(56)$ & $6264(2)$ & 4731(3) & 1526(1) & $25(1)$ \\
\hline$C(57)$ & $6646(2)$ & 4263(3) & $1823(1)$ & $23(1)$ \\
\hline $\mathrm{C}(58)$ & $7299(2)$ & 4846(3) & 1927(1) & $27(1)$ \\
\hline $\mathrm{C}(59)$ & $7564(2)$ & 5872(3) & 1738(1) & $27(1)$ \\
\hline$C(60)$ & $7178(2)$ & 6308(3) & 1447(1) & $22(1)$ \\
\hline$C(61)$ & $5830(2)$ & $3578(3)$ & $2439(1)$ & $28(1)$ \\
\hline$C(62)$ & $5517(2)$ & 2766(4) & $2687(1)$ & $33(1)$ \\
\hline $\mathrm{C}(63)$ & 5181(2) & 3239(4) & 2981(1) & $40(1)$ \\
\hline $\mathrm{C}(64)$ & $5145(2)$ & 4533(4) & $3039(1)$ & $37(1)$ \\
\hline$C(65)$ & $5456(2)$ & $5350(4)$ & 2799(1) & $33(1)$ \\
\hline$C(66)$ & $5789(2)$ & 4884(3) & 2501(1) & $30(1)$ \\
\hline $\mathrm{C}(67)$ & $7076(2)$ & 2135(3) & $2257(1)$ & $28(1)$ \\
\hline $\mathrm{C}(68)$ & $7382(2)$ & 1069(3) & $2100(1)$ & $36(1)$ \\
\hline$C(69)$ & $8009(2)$ & 494(4) & $2240(1)$ & $47(1)$ \\
\hline$C(70)$ & $8335(2)$ & $970(4)$ & $2545(1)$ & $47(1)$ \\
\hline$C(71)$ & $8027(2)$ & 2010(3) & $2705(1)$ & $40(1)$ \\
\hline$C(72)$ & $7414(2)$ & 2584(3) & $2564(1)$ & $33(1)$ \\
\hline $\mathrm{N}(1)$ & 3943(1) & 1514(2) & 792(1) & $21(1)$ \\
\hline$C(73)$ & $4004(2)$ & 1917(3) & $412(1)$ & $28(1)$ \\
\hline$C(74)$ & $4782(2)$ & 1974(3) & $268(1)$ & $35(1)$ \\
\hline$C(75)$ & $4305(2)$ & $222(3)$ & $861(1)$ & $28(1)$ \\
\hline$C(76)$ & $4047(2)$ & $-851(3)$ & $626(1)$ & $36(1)$ \\
\hline$C(77)$ & $3129(2)$ & 1413(3) & $884(1)$ & $24(1)$ \\
\hline$C(78)$ & $2704(2)$ & 2663(3) & $886(1)$ & $34(1)$ \\
\hline$C(79)$ & $4339(2)$ & $2500(3)$ & 1015(1) & $25(1)$ \\
\hline$C(80)$ & $4261(2)$ & 2347(3) & $1405(1)$ & $36(1)$ \\
\hline $\mathrm{N}(2)$ & $8900(2)$ & 3264(3) & 1049(1) & $41(1)$ \\
\hline $\mathrm{C}(81)$ & $9184(3)$ & $3230(6)$ & $1419(2)$ & $117(2)$ \\
\hline$C(82)$ & 9501(4) & 2307(6) & 1593(1) & $146(3)$ \\
\hline $\mathrm{C}(87)$ & $9393(3)$ & $2548(6)$ & $831(2)$ & $176(4)$ \\
\hline $\mathrm{C}(88)$ & 9187(4) & $2556(5)$ & $438(1)$ & $127(3)$ \\
\hline$C(84)$ & $7648(2)$ & 2473(5) & $1225(2)$ & $103(2)$ \\
\hline$C(86)$ & 9161(3) & $5576(5)$ & 919(2) & $173(4)$ \\
\hline$C(83)$ & $8172(3)$ & $2824(12)$ & $1062(2)$ & $333(9)$ \\
\hline$C(85)$ & $8925(7)$ & $4523(5)$ & $940(2)$ & $325(9)$ \\
\hline
\end{tabular}


Table 3. Bond lengths $[\AA]$ and angles $\left[^{\circ}\right]$ for $\left\{\left[\mathrm{NEt}_{4}\right]\left[\mathrm{Ph}_{3} \mathrm{BP}^{m-\mathrm{Ph} 2}\right]\right\}_{2}$.

\begin{tabular}{|c|c|c|c|}
\hline $\mathrm{B}(1)-\mathrm{C}(13)$ & $1.629(4)$ & $\mathrm{C}(26)-\mathrm{C}(27)$ & $1.382(5)$ \\
\hline $\mathrm{B}(1)-\mathrm{C}(7)$ & $1.643(5)$ & $\mathrm{C}(26)-\mathrm{H}(26)$ & 0.9500 \\
\hline $\mathrm{B}(1)-\mathrm{C}(19)$ & $1.644(5)$ & $\mathrm{C}(27)-\mathrm{C}(28)$ & $1.379(5)$ \\
\hline $\mathrm{B}(1)-\mathrm{C}(1)$ & $1.651(5)$ & $\mathrm{C}(27)-\mathrm{H}(27)$ & 0.9500 \\
\hline $\mathrm{P}(1)-\mathrm{C}(31)$ & $1.823(4)$ & $\mathrm{C}(28)-\mathrm{C}(29)$ & $1.370(4)$ \\
\hline$P(1)-C(23)$ & $1.829(3)$ & $\mathrm{C}(28)-\mathrm{H}(28)$ & 0.9500 \\
\hline $\mathrm{P}(1)-\mathrm{C}(25)$ & $1.834(3)$ & $\mathrm{C}(29)-\mathrm{C}(30)$ & $1.376(4)$ \\
\hline$C(1)-C(2)$ & $1.379(4)$ & $\mathrm{C}(29)-\mathrm{H}(29)$ & 0.9500 \\
\hline$C(1)-C(6)$ & $1.395(4)$ & $\mathrm{C}(30)-\mathrm{H}(30)$ & 0.9500 \\
\hline $\mathrm{C}(2)-\mathrm{C}(3)$ & $1.437(5)$ & $C(31)-C(36)$ & $1.393(4)$ \\
\hline $\mathrm{C}(2)-\mathrm{H}(2)$ & 0.9500 & $\mathrm{C}(31)-\mathrm{C}(32)$ & $1.394(5)$ \\
\hline$C(3)-C(4)$ & $1.372(5)$ & $\mathrm{C}(32)-\mathrm{C}(33)$ & $1.379(4)$ \\
\hline $\mathrm{C}(3)-\mathrm{H}(3)$ & 0.9500 & $\mathrm{C}(32)-\mathrm{H}(32)$ & 0.9500 \\
\hline$C(4)-C(5)$ & $1.351(5)$ & C(33)-C(34) & $1.383(5)$ \\
\hline $\mathrm{C}(4)-\mathrm{H}(4)$ & 0.9500 & $\mathrm{C}(33)-\mathrm{H}(33)$ & 0.9500 \\
\hline$C(5)-C(6)$ & $1.390(5)$ & $\mathrm{C}(34)-\mathrm{C}(35)$ & $1.386(5)$ \\
\hline $\mathrm{C}(5)-\mathrm{H}(5)$ & 0.9500 & $\mathrm{C}(34)-\mathrm{H}(34)$ & 0.9500 \\
\hline $\mathrm{C}(6)-\mathrm{H}(6)$ & 0.9500 & C(35)-C(36) & $1.381(5)$ \\
\hline$C(7)-C(12)$ & $1.391(4)$ & $\mathrm{C}(35)-\mathrm{H}(35)$ & 0.9500 \\
\hline $\mathrm{C}(7)-\mathrm{C}(8)$ & $1.397(4)$ & $\mathrm{C}(36)-\mathrm{H}(36)$ & 0.9500 \\
\hline $\mathrm{C}(8)-\mathrm{C}(9)$ & $1.386(4)$ & $B(2)-C(55)$ & $1.636(4)$ \\
\hline $\mathrm{C}(8)-\mathrm{H}(8)$ & 0.9500 & $B(2)-C(43)$ & $1.641(5)$ \\
\hline$C(9)-C(10)$ & $1.371(5)$ & $B(2)-C(49)$ & $1.651(5)$ \\
\hline $\mathrm{C}(9)-\mathrm{H}(9)$ & 0.9500 & $\mathrm{~B}(2)-\mathrm{C}(37)$ & $1.654(4)$ \\
\hline $\mathrm{C}(10)-\mathrm{C}(11)$ & $1.369(5)$ & $\mathrm{P}(2)-\mathrm{C}(61)$ & $1.834(4)$ \\
\hline $\mathrm{C}(10)-\mathrm{H}(10)$ & 0.9500 & $\mathrm{P}(2)-\mathrm{C}(67)$ & $1.843(3)$ \\
\hline $\mathrm{C}(11)-\mathrm{C}(12)$ & $1.393(4)$ & $\mathrm{P}(2)-\mathrm{C}(57)$ & $1.843(3)$ \\
\hline C(11)-H(11) & 0.9500 & C(37)-C(38) & $1.400(4)$ \\
\hline C(12)-H(12) & 0.9500 & $C(37)-C(42)$ & $1.409(4)$ \\
\hline $\mathrm{C}(13)-\mathrm{C}(18)$ & $1.381(4)$ & $\mathrm{C}(38)-\mathrm{C}(39)$ & $1.409(4)$ \\
\hline $\mathrm{C}(13)-\mathrm{C}(14)$ & $1.403(4)$ & $\mathrm{C}(38)-\mathrm{H}(38)$ & 0.9500 \\
\hline C(14)-C(15) & $1.405(4)$ & $C(39)-C(40)$ & $1.377(4)$ \\
\hline C(14)-H(14) & 0.9500 & $\mathrm{C}(39)-\mathrm{H}(39)$ & 0.9500 \\
\hline $\mathrm{C}(15)-\mathrm{C}(16)$ & $1.354(5)$ & $\mathrm{C}(40)-\mathrm{C}(41)$ & $1.376(4)$ \\
\hline $\mathrm{C}(15)-\mathrm{H}(15)$ & 0.9500 & $\mathrm{C}(40)-\mathrm{H}(40)$ & 0.9500 \\
\hline C(16)-C(17) & $1.363(5)$ & $\mathrm{C}(41)-\mathrm{C}(42)$ & $1.388(4)$ \\
\hline $\mathrm{C}(16)-\mathrm{H}(16)$ & 0.9500 & $\mathrm{C}(41)-\mathrm{H}(41)$ & 0.9500 \\
\hline C(17)-C(18) & $1.386(5)$ & $\mathrm{C}(42)-\mathrm{H}(42)$ & 0.9500 \\
\hline C(17)-H(17) & 0.9500 & $\mathrm{C}(43)-\mathrm{C}(48)$ & $1.394(4)$ \\
\hline C(18)-H(18) & 0.9500 & $\mathrm{C}(43)-\mathrm{C}(44)$ & $1.396(4)$ \\
\hline C(19)-C(24) & $1.384(4)$ & $\mathrm{C}(44)-\mathrm{C}(45)$ & $1.398(4)$ \\
\hline$C(19)-C(20)$ & $1.396(4)$ & $\mathrm{C}(44)-\mathrm{H}(44)$ & 0.9500 \\
\hline$C(20)-C(21)$ & $1.389(4)$ & $C(45)-C(46)$ & $1.379(4)$ \\
\hline C(20)-H(20) & 0.9500 & $\mathrm{C}(45)-\mathrm{H}(45)$ & 0.9500 \\
\hline $\mathrm{C}(21)-\mathrm{C}(22)$ & $1.370(4)$ & $\mathrm{C}(46)-\mathrm{C}(47)$ & $1.372(4)$ \\
\hline $\mathrm{C}(21)-\mathrm{H}(21)$ & 0.9500 & $\mathrm{C}(46)-\mathrm{H}(46)$ & 0.9500 \\
\hline$C(22)-C(23)$ & $1.392(4)$ & $\mathrm{C}(47)-\mathrm{C}(48)$ & $1.381(4)$ \\
\hline C(22)-H(22) & 0.9500 & $\mathrm{C}(47)-\mathrm{H}(47)$ & 0.9500 \\
\hline C(23)-C(24) & $1.392(4)$ & $\mathrm{C}(48)-\mathrm{H}(48)$ & 0.9500 \\
\hline $\mathrm{C}(24)-\mathrm{H}(24)$ & 0.9500 & $\mathrm{C}(49)-\mathrm{C}(50)$ & $1.395(4)$ \\
\hline$C(25)-C(26)$ & $1.390(4)$ & $\mathrm{C}(49)-\mathrm{C}(54)$ & $1.397(4)$ \\
\hline$C(25)-C(30)$ & $1.392(4)$ & $\mathrm{C}(50)-\mathrm{C}(51)$ & $1.384(4)$ \\
\hline
\end{tabular}




\begin{tabular}{|c|c|c|c|}
\hline $\mathrm{C}(50)-\mathrm{H}(50)$ & 0.9500 & $C(77)-C(78)$ & $1.519(4)$ \\
\hline C(51)-C(52) & $1.377(5)$ & $\mathrm{C}(77)-\mathrm{H}(77 \mathrm{~A})$ & 0.9900 \\
\hline $\mathrm{C}(51)-\mathrm{H}(51)$ & 0.9500 & $\mathrm{C}(77)-\mathrm{H}(77 \mathrm{~B})$ & 0.9900 \\
\hline C(52)-C(53) & $1.373(4)$ & $\mathrm{C}(78)-\mathrm{H}(78 \mathrm{~A})$ & 0.9800 \\
\hline C(52)-H(52) & 0.9500 & $\mathrm{C}(78)-\mathrm{H}(78 \mathrm{~B})$ & 0.9800 \\
\hline C(53)-C(54) & $1.376(4)$ & $\mathrm{C}(78)-\mathrm{H}(78 \mathrm{C})$ & 0.9800 \\
\hline C(53)-H(53) & 0.9500 & C(79)-C(80) & $1.502(4)$ \\
\hline C(54)-H(54) & 0.9500 & $\mathrm{C}(79)-\mathrm{H}(79 \mathrm{~A})$ & 0.9900 \\
\hline $\mathrm{C}(55)-\mathrm{C}(56)$ & $1.397(4)$ & $\mathrm{C}(79)-\mathrm{H}(79 \mathrm{~B})$ & 0.9900 \\
\hline C(55)-C(60) & $1.401(4)$ & $\mathrm{C}(80)-\mathrm{H}(80 \mathrm{~A})$ & 0.9800 \\
\hline C(56)-C(57) & $1.408(4)$ & $\mathrm{C}(80)-\mathrm{H}(80 \mathrm{~B})$ & 0.9800 \\
\hline C(56)-H(56) & 0.9500 & $\mathrm{C}(80)-\mathrm{H}(80 \mathrm{C})$ & 0.9800 \\
\hline C(57)-C(58) & $1.384(4)$ & $\mathrm{N}(2)-\mathrm{C}(85)$ & $1.387(6)$ \\
\hline C(58)-C(59) & $1.383(4)$ & $\mathrm{N}(2)-\mathrm{C}(83)$ & $1.393(6)$ \\
\hline $\mathrm{C}(58)-\mathrm{H}(58)$ & 0.9500 & $\mathrm{~N}(2)-\mathrm{C}(87)$ & $1.434(6)$ \\
\hline C(59)-C(60) & $1.382(4)$ & $\mathrm{N}(2)-\mathrm{C}(81)$ & $1.495(6)$ \\
\hline C(59)-H(59) & 0.9500 & $\mathrm{C}(81)-\mathrm{C}(82)$ & $1.303(6)$ \\
\hline $\mathrm{C}(60)-\mathrm{H}(60)$ & 0.9500 & $\mathrm{C}(81)-\mathrm{H}(81 \mathrm{~A})$ & 0.9900 \\
\hline C(61)-C(66) & $1.393(4)$ & C(81)-H(81B) & 0.9900 \\
\hline C(61)-C(62) & $1.394(4)$ & $\mathrm{C}(82)-\mathrm{H}(82 \mathrm{~A})$ & 0.9800 \\
\hline $\mathrm{C}(62)-\mathrm{C}(63)$ & $1.371(5)$ & $\mathrm{C}(82)-\mathrm{H}(82 \mathrm{~B})$ & 0.9800 \\
\hline $\mathrm{C}(62)-\mathrm{H}(62)$ & 0.9500 & $\mathrm{C}(82)-\mathrm{H}(82 \mathrm{C})$ & 0.9800 \\
\hline $\mathrm{C}(63)-\mathrm{C}(64)$ & $1.378(5)$ & $\mathrm{C}(87)-\mathrm{C}(88)$ & $1.539(8)$ \\
\hline $\mathrm{C}(63)-\mathrm{H}(63)$ & 0.9500 & $\mathrm{C}(87)-\mathrm{H}(87 \mathrm{~A})$ & 0.9900 \\
\hline $\mathrm{C}(64)-\mathrm{C}(65)$ & $1.374(4)$ & $\mathrm{C}(87)-\mathrm{H}(87 \mathrm{~B})$ & 0.9900 \\
\hline C(64)-H(64) & 0.9500 & $\mathrm{C}(88)-\mathrm{H}(88 \mathrm{~A})$ & 0.9800 \\
\hline C(65)-C(66) & $1.378(4)$ & $\mathrm{C}(88)-\mathrm{H}(88 \mathrm{~B})$ & 0.9800 \\
\hline C(65)-H(65) & 0.9500 & $\mathrm{C}(88)-\mathrm{H}(88 \mathrm{C})$ & 0.9800 \\
\hline C(66)-H(66) & 0.9500 & $\mathrm{C}(84)-\mathrm{C}(83)$ & $1.195(6)$ \\
\hline C(67)-C(68) & $1.386(4)$ & $\mathrm{C}(84)-\mathrm{H}(84 \mathrm{~A})$ & 0.9800 \\
\hline C(67)-C(72) & $1.394(4)$ & C(84)-H(84B) & 0.9800 \\
\hline $\mathrm{C}(68)-\mathrm{C}(69)$ & $1.384(5)$ & $\mathrm{C}(84)-\mathrm{H}(84 \mathrm{C})$ & 0.9800 \\
\hline $\mathrm{C}(68)-\mathrm{H}(68)$ & 0.9500 & $C(86)-C(85)$ & $1.187(8)$ \\
\hline C(69)-C(70) & $1.390(5)$ & $\mathrm{C}(86)-\mathrm{H}(86 \mathrm{~A})$ & 0.9800 \\
\hline C(69)-H(69) & 0.9500 & $\mathrm{C}(86)-\mathrm{H}(86 \mathrm{~B})$ & 0.9800 \\
\hline C(70)-C(71) & $1.371(5)$ & $\mathrm{C}(86)-\mathrm{H}(86 \mathrm{C})$ & 0.9800 \\
\hline $\mathrm{C}(70)-\mathrm{H}(70)$ & 0.9500 & $\mathrm{C}(83)-\mathrm{H}(83 \mathrm{~A})$ & 0.9900 \\
\hline C(71)-C(72) & $1.366(4)$ & $\mathrm{C}(83)-\mathrm{H}(83 \mathrm{~B})$ & 0.9900 \\
\hline $\mathrm{C}(71)-\mathrm{H}(71)$ & 0.9500 & $\mathrm{C}(85)-\mathrm{H}(85 \mathrm{~A})$ & 0.9900 \\
\hline $\mathrm{C}(72)-\mathrm{H}(72)$ & 0.9500 & $\mathrm{C}(85)-\mathrm{H}(85 \mathrm{~B})$ & 0.9900 \\
\hline $\mathrm{N}(1)-\mathrm{C}(79)$ & $1.513(4)$ & & \\
\hline $\mathrm{N}(1)-\mathrm{C}(73)$ & $1.514(4)$ & $\mathrm{C}(13)-\mathrm{B}(1)-\mathrm{C}(7)$ & $113.9(3)$ \\
\hline $\mathrm{N}(1)-\mathrm{C}(77)$ & $1.517(4)$ & $\mathrm{C}(13)-\mathrm{B}(1)-\mathrm{C}(19)$ & $112.8(3)$ \\
\hline $\mathrm{N}(1)-\mathrm{C}(75)$ & $1.526(4)$ & $\mathrm{C}(7)-\mathrm{B}(1)-\mathrm{C}(19)$ & $102.8(2)$ \\
\hline C(73)-C(74) & $1.514(4)$ & $\mathrm{C}(13)-\mathrm{B}(1)-\mathrm{C}(1)$ & $103.6(2)$ \\
\hline $\mathrm{C}(73)-\mathrm{H}(73 \mathrm{~A})$ & 0.9900 & $\mathrm{C}(7)-\mathrm{B}(1)-\mathrm{C}(1)$ & $110.9(3)$ \\
\hline $\mathrm{C}(73)-\mathrm{H}(73 \mathrm{~B})$ & 0.9900 & $\mathrm{C}(19)-\mathrm{B}(1)-\mathrm{C}(1)$ & $113.2(3)$ \\
\hline $\mathrm{C}(74)-\mathrm{H}(74 \mathrm{~A})$ & 0.9800 & $\mathrm{C}(31)-\mathrm{P}(1)-\mathrm{C}(23)$ & $101.44(15)$ \\
\hline C(74)-H(74B) & 0.9800 & $\mathrm{C}(31)-\mathrm{P}(1)-\mathrm{C}(25)$ & $100.88(16)$ \\
\hline $\mathrm{C}(74)-\mathrm{H}(74 \mathrm{C})$ & 0.9800 & $\mathrm{C}(23)-\mathrm{P}(1)-\mathrm{C}(25)$ & $104.07(15)$ \\
\hline$C(75)-C(76)$ & $1.508(4)$ & $C(2)-C(1)-C(6)$ & $115.9(3)$ \\
\hline $\mathrm{C}(75)-\mathrm{H}(75 \mathrm{~A})$ & 0.9900 & $\mathrm{C}(2)-\mathrm{C}(1)-\mathrm{B}(1)$ & $123.0(3)$ \\
\hline C(75)-H(75B) & 0.9900 & $\mathrm{C}(6)-\mathrm{C}(1)-\mathrm{B}(1)$ & $120.9(3)$ \\
\hline $\mathrm{C}(76)-\mathrm{H}(76 \mathrm{~A})$ & 0.9800 & $\mathrm{C}(1)-\mathrm{C}(2)-\mathrm{C}(3)$ & 121.3(4) \\
\hline $\mathrm{C}(76)-\mathrm{H}(76 \mathrm{~B})$ & 0.9800 & $\mathrm{C}(1)-\mathrm{C}(2)-\mathrm{H}(2)$ & 119.3 \\
\hline $\mathrm{C}(76)-\mathrm{H}(76 \mathrm{C})$ & 0.9800 & $\mathrm{C}(3)-\mathrm{C}(2)-\mathrm{H}(2)$ & 119.3 \\
\hline
\end{tabular}




\begin{tabular}{|c|c|}
\hline $\mathrm{C}(4)-\mathrm{C}(3)-\mathrm{C}(2)$ & $118.2(4)$ \\
\hline $\mathrm{C}(4)-\mathrm{C}(3)-\mathrm{H}(3)$ & 120.9 \\
\hline $\mathrm{C}(2)-\mathrm{C}(3)-\mathrm{H}(3)$ & 120.9 \\
\hline $\mathrm{C}(5)-\mathrm{C}(4)-\mathrm{C}(3)$ & $122.8(4)$ \\
\hline $\mathrm{C}(5)-\mathrm{C}(4)-\mathrm{H}(4)$ & 118.6 \\
\hline $\mathrm{C}(3)-\mathrm{C}(4)-\mathrm{H}(4)$ & 118.6 \\
\hline $\mathrm{C}(4)-\mathrm{C}(5)-\mathrm{C}(6)$ & $117.4(4)$ \\
\hline $\mathrm{C}(4)-\mathrm{C}(5)-\mathrm{H}(5)$ & 121.3 \\
\hline $\mathrm{C}(6)-\mathrm{C}(5)-\mathrm{H}(5)$ & 121.3 \\
\hline $\mathrm{C}(5)-\mathrm{C}(6)-\mathrm{C}(1)$ & $124.4(4)$ \\
\hline $\mathrm{C}(5)-\mathrm{C}(6)-\mathrm{H}(6)$ & 117.8 \\
\hline $\mathrm{C}(1)-\mathrm{C}(6)-\mathrm{H}(6)$ & 117.8 \\
\hline $\mathrm{C}(12)-\mathrm{C}(7)-\mathrm{C}(8)$ & $114.5(3)$ \\
\hline $\mathrm{C}(12)-\mathrm{C}(7)-\mathrm{B}(1)$ & 123.1(3) \\
\hline $\mathrm{C}(8)-\mathrm{C}(7)-\mathrm{B}(1)$ & 122.1(3) \\
\hline $\mathrm{C}(9)-\mathrm{C}(8)-\mathrm{C}(7)$ & $123.2(3)$ \\
\hline $\mathrm{C}(9)-\mathrm{C}(8)-\mathrm{H}(8)$ & 118.4 \\
\hline $\mathrm{C}(7)-\mathrm{C}(8)-\mathrm{H}(8)$ & 118.4 \\
\hline $\mathrm{C}(10)-\mathrm{C}(9)-\mathrm{C}(8)$ & $119.8(4)$ \\
\hline $\mathrm{C}(10)-\mathrm{C}(9)-\mathrm{H}(9)$ & 120.1 \\
\hline $\mathrm{C}(8)-\mathrm{C}(9)-\mathrm{H}(9)$ & 120.1 \\
\hline$C(11)-C(10)-C(9)$ & $119.6(4)$ \\
\hline $\mathrm{C}(11)-\mathrm{C}(10)-\mathrm{H}(10)$ & 120.2 \\
\hline $\mathrm{C}(9)-\mathrm{C}(10)-\mathrm{H}(10)$ & 120.2 \\
\hline $\mathrm{C}(10)-\mathrm{C}(11)-\mathrm{C}(12)$ & $119.6(4)$ \\
\hline $\mathrm{C}(10)-\mathrm{C}(11)-\mathrm{H}(11)$ & 120.2 \\
\hline $\mathrm{C}(12)-\mathrm{C}(11)-\mathrm{H}(11)$ & 120.2 \\
\hline $\mathrm{C}(7)-\mathrm{C}(12)-\mathrm{C}(11)$ & $123.3(4)$ \\
\hline $\mathrm{C}(7)-\mathrm{C}(12)-\mathrm{H}(12)$ & 118.3 \\
\hline $\mathrm{C}(11)-\mathrm{C}(12)-\mathrm{H}(12)$ & 118.3 \\
\hline $\mathrm{C}(18)-\mathrm{C}(13)-\mathrm{C}(14)$ & $114.0(3)$ \\
\hline $\mathrm{C}(18)-\mathrm{C}(13)-\mathrm{B}(1)$ & $122.0(3)$ \\
\hline $\mathrm{C}(14)-\mathrm{C}(13)-\mathrm{B}(1)$ & $123.6(3)$ \\
\hline$C(13)-C(14)-C(15)$ & $122.5(4)$ \\
\hline $\mathrm{C}(13)-\mathrm{C}(14)-\mathrm{H}(14)$ & 118.7 \\
\hline $\mathrm{C}(15)-\mathrm{C}(14)-\mathrm{H}(14)$ & 118.7 \\
\hline$C(16)-C(15)-C(14)$ & $119.7(4)$ \\
\hline $\mathrm{C}(16)-\mathrm{C}(15)-\mathrm{H}(15)$ & 120.1 \\
\hline $\mathrm{C}(14)-\mathrm{C}(15)-\mathrm{H}(15)$ & 120.1 \\
\hline$C(15)-C(16)-C(17)$ & $120.2(4)$ \\
\hline $\mathrm{C}(15)-\mathrm{C}(16)-\mathrm{H}(16)$ & 119.9 \\
\hline $\mathrm{C}(17)-\mathrm{C}(16)-\mathrm{H}(16)$ & 119.9 \\
\hline $\mathrm{C}(16)-\mathrm{C}(17)-\mathrm{C}(18)$ & $119.2(4)$ \\
\hline $\mathrm{C}(16)-\mathrm{C}(17)-\mathrm{H}(17)$ & 120.4 \\
\hline $\mathrm{C}(18)-\mathrm{C}(17)-\mathrm{H}(17)$ & 120.4 \\
\hline $\mathrm{C}(13)-\mathrm{C}(18)-\mathrm{C}(17)$ & $124.3(4)$ \\
\hline $\mathrm{C}(13)-\mathrm{C}(18)-\mathrm{H}(18)$ & 117.8 \\
\hline $\mathrm{C}(17)-\mathrm{C}(18)-\mathrm{H}(18)$ & 117.8 \\
\hline $\mathrm{C}(24)-\mathrm{C}(19)-\mathrm{C}(20)$ & $115.0(3)$ \\
\hline $\mathrm{C}(24)-\mathrm{C}(19)-\mathrm{B}(1)$ & $120.7(3)$ \\
\hline $\mathrm{C}(20)-\mathrm{C}(19)-\mathrm{B}(1)$ & $124.0(3)$ \\
\hline $\mathrm{C}(21)-\mathrm{C}(20)-\mathrm{C}(19)$ & $122.7(3)$ \\
\hline $\mathrm{C}(21)-\mathrm{C}(20)-\mathrm{H}(20)$ & 118.6 \\
\hline $\mathrm{C}(19)-\mathrm{C}(20)-\mathrm{H}(20)$ & 118.6 \\
\hline $\mathrm{C}(22)-\mathrm{C}(21)-\mathrm{C}(20)$ & $119.7(3)$ \\
\hline $\mathrm{C}(22)-\mathrm{C}(21)-\mathrm{H}(21)$ & 120.1 \\
\hline
\end{tabular}

\begin{tabular}{|c|c|}
\hline $\mathrm{C}(20)-\mathrm{C}(21)-\mathrm{H}(21)$ & 120.1 \\
\hline $\mathrm{C}(21)-\mathrm{C}(22)-\mathrm{C}(23)$ & $120.4(3)$ \\
\hline $\mathrm{C}(21)-\mathrm{C}(22)-\mathrm{H}(22)$ & 119.8 \\
\hline $\mathrm{C}(23)-\mathrm{C}(22)-\mathrm{H}(22)$ & 119.8 \\
\hline $\mathrm{C}(22)-\mathrm{C}(23)-\mathrm{C}(24)$ & $117.7(3)$ \\
\hline $\mathrm{C}(22)-\mathrm{C}(23)-\mathrm{P}(1)$ & $126.4(3)$ \\
\hline $\mathrm{C}(24)-\mathrm{C}(23)-\mathrm{P}(1)$ & $115.9(2)$ \\
\hline$C(19)-C(24)-C(23)$ & $124.5(3)$ \\
\hline $\mathrm{C}(19)-\mathrm{C}(24)-\mathrm{H}(24)$ & 117.8 \\
\hline $\mathrm{C}(23)-\mathrm{C}(24)-\mathrm{H}(24)$ & 117.8 \\
\hline $\mathrm{C}(26)-\mathrm{C}(25)-\mathrm{C}(30)$ & $118.7(3)$ \\
\hline $\mathrm{C}(26)-\mathrm{C}(25)-\mathrm{P}(1)$ & $117.5(3)$ \\
\hline $\mathrm{C}(30)-\mathrm{C}(25)-\mathrm{P}(1)$ & $123.7(3)$ \\
\hline $\mathrm{C}(27)-\mathrm{C}(26)-\mathrm{C}(25)$ & $120.1(3)$ \\
\hline $\mathrm{C}(27)-\mathrm{C}(26)-\mathrm{H}(26)$ & 119.9 \\
\hline $\mathrm{C}(25)-\mathrm{C}(26)-\mathrm{H}(26)$ & 119.9 \\
\hline $\mathrm{C}(28)-\mathrm{C}(27)-\mathrm{C}(26)$ & $120.1(4)$ \\
\hline $\mathrm{C}(28)-\mathrm{C}(27)-\mathrm{H}(27)$ & 119.9 \\
\hline $\mathrm{C}(26)-\mathrm{C}(27)-\mathrm{H}(27)$ & 119.9 \\
\hline $\mathrm{C}(29)-\mathrm{C}(28)-\mathrm{C}(27)$ & $120.3(4)$ \\
\hline $\mathrm{C}(29)-\mathrm{C}(28)-\mathrm{H}(28)$ & 119.9 \\
\hline $\mathrm{C}(27)-\mathrm{C}(28)-\mathrm{H}(28)$ & 119.9 \\
\hline $\mathrm{C}(28)-\mathrm{C}(29)-\mathrm{C}(30)$ & $120.0(4)$ \\
\hline $\mathrm{C}(28)-\mathrm{C}(29)-\mathrm{H}(29)$ & 120.0 \\
\hline $\mathrm{C}(30)-\mathrm{C}(29)-\mathrm{H}(29)$ & 120.0 \\
\hline $\mathrm{C}(29)-\mathrm{C}(30)-\mathrm{C}(25)$ & $120.7(3)$ \\
\hline $\mathrm{C}(29)-\mathrm{C}(30)-\mathrm{H}(30)$ & 119.6 \\
\hline $\mathrm{C}(25)-\mathrm{C}(30)-\mathrm{H}(30)$ & 119.6 \\
\hline $\mathrm{C}(36)-\mathrm{C}(31)-\mathrm{C}(32)$ & $116.4(4)$ \\
\hline $\mathrm{C}(36)-\mathrm{C}(31)-\mathrm{P}(1)$ & $118.5(3)$ \\
\hline $\mathrm{C}(32)-\mathrm{C}(31)-\mathrm{P}(1)$ & $125.1(3)$ \\
\hline $\mathrm{C}(33)-\mathrm{C}(32)-\mathrm{C}(31)$ & $122.1(4)$ \\
\hline $\mathrm{C}(33)-\mathrm{C}(32)-\mathrm{H}(32)$ & 118.9 \\
\hline $\mathrm{C}(31)-\mathrm{C}(32)-\mathrm{H}(32)$ & 118.9 \\
\hline $\mathrm{C}(32)-\mathrm{C}(33)-\mathrm{C}(34)$ & $120.5(4)$ \\
\hline $\mathrm{C}(32)-\mathrm{C}(33)-\mathrm{H}(33)$ & 119.7 \\
\hline $\mathrm{C}(34)-\mathrm{C}(33)-\mathrm{H}(33)$ & 119.7 \\
\hline $\mathrm{C}(33)-\mathrm{C}(34)-\mathrm{C}(35)$ & $118.4(4)$ \\
\hline $\mathrm{C}(33)-\mathrm{C}(34)-\mathrm{H}(34)$ & 120.8 \\
\hline $\mathrm{C}(35)-\mathrm{C}(34)-\mathrm{H}(34)$ & 120.8 \\
\hline $\mathrm{C}(36)-\mathrm{C}(35)-\mathrm{C}(34)$ & $120.6(4)$ \\
\hline $\mathrm{C}(36)-\mathrm{C}(35)-\mathrm{H}(35)$ & 119.7 \\
\hline $\mathrm{C}(34)-\mathrm{C}(35)-\mathrm{H}(35)$ & 119.7 \\
\hline $\mathrm{C}(35)-\mathrm{C}(36)-\mathrm{C}(31)$ & $121.9(4)$ \\
\hline $\mathrm{C}(35)-\mathrm{C}(36)-\mathrm{H}(36)$ & 119.1 \\
\hline $\mathrm{C}(31)-\mathrm{C}(36)-\mathrm{H}(36)$ & 119.1 \\
\hline$C(55)-B(2)-C(43)$ & 111.4(3) \\
\hline $\mathrm{C}(55)-\mathrm{B}(2)-\mathrm{C}(49)$ & $104.5(2)$ \\
\hline $\mathrm{C}(43)-\mathrm{B}(2)-\mathrm{C}(49)$ & $112.1(3)$ \\
\hline $\mathrm{C}(55)-\mathrm{B}(2)-\mathrm{C}(37)$ & $115.1(3)$ \\
\hline $\mathrm{C}(43)-\mathrm{B}(2)-\mathrm{C}(37)$ & 104.1(3) \\
\hline $\mathrm{C}(49)-\mathrm{B}(2)-\mathrm{C}(37)$ & $109.8(3)$ \\
\hline $\mathrm{C}(61)-\mathrm{P}(2)-\mathrm{C}(67)$ & $99.36(15)$ \\
\hline $\mathrm{C}(61)-\mathrm{P}(2)-\mathrm{C}(57)$ & $102.65(16)$ \\
\hline $\mathrm{C}(67)-\mathrm{P}(2)-\mathrm{C}(57)$ & $102.81(15)$ \\
\hline $\mathrm{C}(38)-\mathrm{C}(37)-\mathrm{C}(42)$ & $114.9(3)$ \\
\hline
\end{tabular}




$\begin{array}{ll}\mathrm{C}(38)-\mathrm{C}(37)-\mathrm{B}(2) & 122.6(3) \\ \mathrm{C}(42)-\mathrm{C}(37)-\mathrm{B}(2) & 121.9(3) \\ \mathrm{C}(37)-\mathrm{C}(38)-\mathrm{C}(39) & 122.6(3) \\ \mathrm{C}(37)-\mathrm{C}(38)-\mathrm{H}(38) & 118.7 \\ \mathrm{C}(39)-\mathrm{C}(38)-\mathrm{H}(38) & 118.7 \\ \mathrm{C}(40)-\mathrm{C}(39)-\mathrm{C}(38) & 119.4(3) \\ \mathrm{C}(40)-\mathrm{C}(39)-\mathrm{H}(39) & 120.3 \\ \mathrm{C}(38)-\mathrm{C}(39)-\mathrm{H}(39) & 120.3 \\ \mathrm{C}(41)-\mathrm{C}(40)-\mathrm{C}(39) & 120.3(3) \\ \mathrm{C}(41)-\mathrm{C}(40)-\mathrm{H}(40) & 119.9 \\ \mathrm{C}(39)-\mathrm{C}(40)-\mathrm{H}(40) & 119.9 \\ \mathrm{C}(40)-\mathrm{C}(41)-\mathrm{C}(42) & 119.5(3) \\ \mathrm{C}(40)-\mathrm{C}(41)-\mathrm{H}(41) & 120.3 \\ \mathrm{C}(42)-\mathrm{C}(41)-\mathrm{H}(41) & 120.3 \\ \mathrm{C}(41)-\mathrm{C}(42)-\mathrm{C}(37) & 123.3(3) \\ \mathrm{C}(41)-\mathrm{C}(42)-\mathrm{H}(42) & 118.4 \\ \mathrm{C}(37)-\mathrm{C}(42)-\mathrm{H}(42) & 118.4 \\ \mathrm{C}(48)-\mathrm{C}(43)-\mathrm{C}(44) & 114.8(3) \\ \mathrm{C}(48)-\mathrm{C}(43)-\mathrm{B}(2) & 121.7(3) \\ \mathrm{C}(44)-\mathrm{C}(43)-\mathrm{B}(2) & 123.4(3) \\ \mathrm{C}(43)-\mathrm{C}(44)-\mathrm{C}(45) & 122.3(3) \\ \mathrm{C}(43)-\mathrm{C}(44)-\mathrm{H}(44) & 118.9 \\ \mathrm{C}(45)-\mathrm{C}(44)-\mathrm{H}(44) & 118.9 \\ \mathrm{C}(46)-\mathrm{C}(45)-\mathrm{C}(44) & 120.2(3) \\ \mathrm{C}(46)-\mathrm{C}(45)-\mathrm{H}(45) & 119.9 \\ \mathrm{C}(44)-\mathrm{C}(45)-\mathrm{H}(45) & 119.9 \\ \mathrm{C}(47)-\mathrm{C}(46)-\mathrm{C}(45) & 119.1(3) \\ \mathrm{C}(47)-\mathrm{C}(46)-\mathrm{H}(46) & 120.4 \\ \mathrm{C}(45)-\mathrm{C}(46)-\mathrm{H}(46) & 120.4 \\ \mathrm{C}(46)-\mathrm{C}(47)-\mathrm{C}(48) & 119.8(3) \\ \mathrm{C}(46)-\mathrm{C}(47)-\mathrm{H}(47) & 120.1 \\ \mathrm{C}(48)-\mathrm{C}(47)-\mathrm{H}(47) & 120.1 \\ \mathrm{C}(47)-\mathrm{C}(48)-\mathrm{C}(43) & 123.7(3) \\ \mathrm{C}(47)-\mathrm{C}(48)-\mathrm{H}(48) & 118.1 \\ \mathrm{C}(43)-\mathrm{C}(48)-\mathrm{H}(48) & 118.1 \\ \mathrm{C}(50)-\mathrm{C}(49)-\mathrm{C}(54) & 114.7(3) \\ \mathrm{C}(50)-\mathrm{C}(49)-\mathrm{B}(2) & 124.0(3) \\ \mathrm{C}(54)-\mathrm{C}(49)-\mathrm{B}(2) & 121.1(3) \\ \mathrm{C}(51)-\mathrm{C}(50)-\mathrm{C}(49) & 122.4(3) \\ \mathrm{C}(51)-\mathrm{C}(50)-\mathrm{H}(50) & 118.8 \\ \mathrm{C}(49)-\mathrm{C}(50)-\mathrm{H}(50) & 118.8 \\ \mathrm{C}(52)-\mathrm{C}(51)-\mathrm{C}(50) & 120.8(3) \\ \mathrm{C}(52)-\mathrm{C}(51)-\mathrm{H}(51) & 119.6 \\ \mathrm{C}(50)-\mathrm{C}(51)-\mathrm{H}(51) & 119.6 \\ \mathrm{C}(53)-\mathrm{C}(52)-\mathrm{C}(51) & 118.3(3) \\ \mathrm{C}(53)-\mathrm{C}(52)-\mathrm{H}(52) & 120.9 \\ \mathrm{C}(51)-\mathrm{C}(52)-\mathrm{H}(52) & 120.9 \\ \mathrm{C}(52)-\mathrm{C}(53)-\mathrm{C}(54) & 120.5(3) \\ \mathrm{C}(52)-\mathrm{C}(53)-\mathrm{H}(53) & 119.8 \\ \mathrm{C}(54)-\mathrm{C}(53)-\mathrm{H}(53) & 119.8 \\ \mathrm{C}(53)-\mathrm{C}(54)-\mathrm{C}(49) & 123.2(3) \\ \mathrm{C}(53)-\mathrm{C}(54)-\mathrm{H}(54) & 118.4 \\ \mathrm{C}(49)-\mathrm{C}(54)-\mathrm{H}(54) & 118.4 \\ \mathrm{C}(56)-\mathrm{C}(55)-\mathrm{C}(60) & 114.9(3) \\ \mathrm{C}(56)-\mathrm{C}(55)-\mathrm{B}(2) & 125.4(3) \\ \mathrm{C}(60)-\mathrm{C}(55)-\mathrm{B}(2) & \\ & \end{array}$

$\begin{array}{ll} & \\ \mathrm{C}(55)-\mathrm{C}(56)-\mathrm{C}(57) & 123.1(3) \\ \mathrm{C}(55)-\mathrm{C}(56)-\mathrm{H}(56) & 118.5 \\ \mathrm{C}(57)-\mathrm{C}(56)-\mathrm{H}(56) & 118.5 \\ \mathrm{C}(58)-\mathrm{C}(57)-\mathrm{C}(56) & 119.2(3) \\ \mathrm{C}(58)-\mathrm{C}(57)-\mathrm{P}(2) & 123.1(3) \\ \mathrm{C}(56)-\mathrm{C}(57)-\mathrm{P}(2) & 117.7(3) \\ \mathrm{C}(59)-\mathrm{C}(58)-\mathrm{C}(57) & 119.5(3) \\ \mathrm{C}(59)-\mathrm{C}(58)-\mathrm{H}(58) & 120.3 \\ \mathrm{C}(57)-\mathrm{C}(58)-\mathrm{H}(58) & 120.3 \\ \mathrm{C}(60)-\mathrm{C}(59)-\mathrm{C}(58) & 120.1(3) \\ \mathrm{C}(60)-\mathrm{C}(59)-\mathrm{H}(59) & 119.9 \\ \mathrm{C}(58)-\mathrm{C}(59)-\mathrm{H}(59) & 119.9 \\ \mathrm{C}(59)-\mathrm{C}(60)-\mathrm{C}(55) & 123.3(3) \\ \mathrm{C}(59)-\mathrm{C}(60)-\mathrm{H}(60) & 118.4 \\ \mathrm{C}(55)-\mathrm{C}(60)-\mathrm{H}(60) & 118.4 \\ \mathrm{C}(66)-\mathrm{C}(61)-\mathrm{C}(62) & 117.7(3) \\ \mathrm{C}(66)-\mathrm{C}(61)-\mathrm{P}(2) & 124.1(3) \\ \mathrm{C}(62)-\mathrm{C}(61)-\mathrm{P}(2) & 118.2(3) \\ \mathrm{C}(63)-\mathrm{C}(62)-\mathrm{C}(61) & 121.0(4) \\ \mathrm{C}(63)-\mathrm{C}(62)-\mathrm{H}(62) & 119.5 \\ \mathrm{C}(61)-\mathrm{C}(62)-\mathrm{H}(62) & 119.5 \\ \mathrm{C}(62)-\mathrm{C}(63)-\mathrm{C}(64) & 120.6(4) \\ \mathrm{C}(62)-\mathrm{C}(63)-\mathrm{H}(63) & 119.7 \\ \mathrm{C}(64)-\mathrm{C}(63)-\mathrm{H}(63) & 119.7 \\ \mathrm{C}(65)-\mathrm{C}(64)-\mathrm{C}(63) & 119.3(4) \\ \mathrm{C}(65)-\mathrm{C}(64)-\mathrm{H}(64) & 120.3 \\ \mathrm{C}(63)-\mathrm{C}(64)-\mathrm{H}(64) & 120.3 \\ \mathrm{C}(64)-\mathrm{C}(65)-\mathrm{C}(66) & 120.5(4) \\ \mathrm{C}(64)-\mathrm{C}(65)-\mathrm{H}(65) & 119.7 \\ \mathrm{C}(66)-\mathrm{C}(65)-\mathrm{H}(65) & 119.7 \\ \mathrm{C}(65)-\mathrm{C}(66)-\mathrm{C}(61) & 120.9(3) \\ \mathrm{C}(65)-\mathrm{C}(66)-\mathrm{H}(66) & 119.6 \\ \mathrm{C}(61)-\mathrm{C}(66)-\mathrm{H}(66) & 119.6 \\ \mathrm{C}(68)-\mathrm{C}(67)-\mathrm{C}(72) & 117.4(3) \\ \mathrm{C}(68)-\mathrm{C}(67)-\mathrm{P}(2) & 118.3(3) \\ \mathrm{C}(72)-\mathrm{C}(67)-\mathrm{P}(2) & 124.3(3) \\ \mathrm{C}(69)-\mathrm{C}(68)-\mathrm{C}(67) & 120.9(4) \\ \mathrm{C}(69)-\mathrm{C}(68)-\mathrm{H}(68) & 119.5 \\ \mathrm{C}(67)-\mathrm{C}(68)-\mathrm{H}(68) & 119.5 \\ \mathrm{C}(68)-\mathrm{C}(69)-\mathrm{C}(70) & 120.2(4) \\ \mathrm{C}(68)-\mathrm{C}(69)-\mathrm{H}(69) & 119.9 \\ \mathrm{C}(70)-\mathrm{C}(69)-\mathrm{H}(69) & 119.9 \\ \mathrm{C}(71)-\mathrm{C}(70)-\mathrm{C}(69) & 119.2(4) \\ \mathrm{C}(71)-\mathrm{C}(70)-\mathrm{H}(70) & 120.4 \\ \mathrm{C}(69)-\mathrm{C}(70)-\mathrm{H}(70) & 120.4 \\ \mathrm{C}(72)-\mathrm{C}(71)-\mathrm{C}(70) & 120.4(4) \\ \mathrm{C}(72)-\mathrm{C}(71)-\mathrm{H}(71) & 119.8 \\ \mathrm{C}(70)-\mathrm{C}(71)-\mathrm{H}(71) & 119.8 \\ \mathrm{C}(71)-\mathrm{C}(72)-\mathrm{C}(67) & 121.9(4) \\ \mathrm{C}(71)-\mathrm{C}(72)-\mathrm{H}(72) & 119.1 \\ \mathrm{C}(67)-\mathrm{C}(72)-\mathrm{H}(72) & 119.1 \\ \mathrm{C}(79)-\mathrm{N}(1)-\mathrm{C}(73) & 107.9(2) \\ \mathrm{C}(79)-\mathrm{N}(1)-\mathrm{C}(77) & 111.9(2) \\ \mathrm{C}(73)-\mathrm{N}(1)-\mathrm{C}(77) & 108.6(2) \\ \mathrm{C}(79)-\mathrm{N}(1)-\mathrm{C}(75) & 108.2(2) \\ \mathrm{C}(73)-\mathrm{N}(1)-\mathrm{C}(75) & 112.2(2) \\ & \\ & \end{array}$




$\begin{array}{ll}\text { C(77)-N(1)-C(75) } & 108.2(2) \\ \text { N(1)-C(73)-C(74) } & 115.7(3) \\ \text { N(1)-C(73)-H(73A) } & 108.4 \\ \text { C(74)-C(73)-H(73A) } & 108.4 \\ \text { N(1)-C(73)-H(73B) } & 108.4 \\ \text { C(74)-C(73)-H(73B) } & 108.4 \\ \text { H(73A)-C(73)-H(73B) } & 107.4 \\ \text { C(73)-C(74)-H(74A) } & 109.5 \\ \text { C(73)-C(74)-H(74B) } & 109.5 \\ \text { H(74A)-C(74)-H(74B) } & 109.5 \\ \text { C(73)-C(74)-H(74C) } & 109.5 \\ \text { H(74A)-C(74)-H(74C) } & 109.5 \\ \text { H(74B)-C(74)-H(74C) } & 109.5 \\ \text { C(76)-C(75)-N(1) } & 115.7(3) \\ \text { C(76)-C(75)-H(75A) } & 108.4 \\ \text { N(1)-C(75)-H(75A) } & 108.4 \\ \text { C(76)-C(75)-H(75B) } & 108.4 \\ \text { N(1)-C(75)-H(75B) } & 108.4 \\ \text { H(75A)-C(75)-H(75B) } & 107.4 \\ \text { C(75)-C(76)-H(76A) } & 109.5 \\ \text { C(75)-C(76)-H(76B) } & 109.5 \\ \text { H(76A)-C(76)-H(76B) } & 109.5 \\ \text { C(75)-C(76)-H(76C) } & 109.5 \\ \text { H(76A)-C(76)-H(76C) } & 109.5 \\ \text { H(76B)-C(76)-H(76C) } & 109.5 \\ \text { N(1)-C(77)-C(78) } & 115.6(3) \\ \text { N(1)-C(77)-H(77A) } & 108.4 \\ \text { C(78)-C(77)-H(77A) } & 108.4 \\ \text { N(1)-C(77)-H(77B) } & 108.4 \\ \text { C(78)-C(77)-H(77B) } & 108.4 \\ \text { H(77A)-C(77)-H(77B) } & 107.5 \\ \text { C(77)-C(78)-H(78A) } & 109.5 \\ \text { C(77)-C(78)-H(78B) } & 109.5 \\ \text { H(78A)-C(78)-H(78B) } & 109.5 \\ \text { C(77)-C(78)-H(78C) } & 109.5 \\ \text { H(78A)-C(78)-H(78C) } & 109.5 \\ \text { H(78B)-C(78)-H(78C) } & 109.5 \\ \text { C(80)-C(79)-N(1) } & 115.7(3) \\ \text { C(80)-C(79)-H(79A) } & 108.4 \\ \text { N(1)-C(79)-H(79A) } & 108.4 \\ \text { C(80)-C(79)-H(79B) } & 108.4 \\ \text { N(1)-C(79)-H(79B) } & 108.4 \\ \text { H(79A)-C(79)-H(79B) } & 107.4 \\ \text { C(79)-C(80)-H(80A) } & 109.5 \\ \text { C(79)-C(80)-H(80B) } & 109.5 \\ \text { H(80A)-C(80)-H(80B) } & 109.5 \\ \text { C(79)-C(80)-H(80C) } & 109.5 \\ \text { H(80A)-C(80)-H(80C) } & 109.5 \\ \text { H(80B)-C(80)-H(80C) } & 109.5 \\ \text { C(85)-N(2)-C(83) } & 111.0(7) \\ \text { C(85)-N(2)-C(87) } & 107.7(5) \\ \text { C(83)-N(2)-C(87) } & 115.8(6) \\ & \\ & \end{array}$

$\begin{array}{lc} & \\ \mathrm{C}(85)-\mathrm{N}(2)-\mathrm{C}(81) & 107.1(5) \\ \mathrm{C}(83)-\mathrm{N}(2)-\mathrm{C}(81) & 106.0(4) \\ \mathrm{C}(87)-\mathrm{N}(2)-\mathrm{C}(81) & 108.9(5) \\ \mathrm{C}(82)-\mathrm{C}(81)-\mathrm{N}(2) & 129.9(5) \\ \mathrm{C}(82)-\mathrm{C}(81)-\mathrm{H}(81 \mathrm{~A}) & 104.8 \\ \mathrm{~N}(2)-\mathrm{C}(81)-\mathrm{H}(81 \mathrm{~A}) & 104.8 \\ \mathrm{C}(82)-\mathrm{C}(81)-\mathrm{H}(81 \mathrm{~B}) & 104.8 \\ \mathrm{~N}(2)-\mathrm{C}(81)-\mathrm{H}(81 \mathrm{~B}) & 104.8 \\ \mathrm{H}(81 \mathrm{~A})-\mathrm{C}(81)-\mathrm{H}(81 \mathrm{~B}) & 105.8 \\ \mathrm{C}(81)-\mathrm{C}(82)-\mathrm{H}(82 \mathrm{~A}) & 109.5 \\ \mathrm{C}(81)-\mathrm{C}(82)-\mathrm{H}(82 \mathrm{~B}) & 109.5 \\ \mathrm{H}(82 \mathrm{~A})-\mathrm{C}(82)-\mathrm{H}(82 \mathrm{~B}) & 109.5 \\ \mathrm{C}(81)-\mathrm{C}(82)-\mathrm{H}(82 \mathrm{C}) & 109.5 \\ \mathrm{H}(82 \mathrm{~A})-\mathrm{C}(82)-\mathrm{H}(82 \mathrm{C}) & 109.5 \\ \mathrm{H}(82 \mathrm{~B})-\mathrm{C}(82)-\mathrm{H}(82 \mathrm{C}) & 109.5 \\ \mathrm{~N}(2)-\mathrm{C}(87)-\mathrm{C}(88) & 114.4(5) \\ \mathrm{N}(2)-\mathrm{C}(87)-\mathrm{H}(87 \mathrm{~A}) & 108.6 \\ \mathrm{C}(88)-\mathrm{C}(87)-\mathrm{H}(87 \mathrm{~A}) & 108.6 \\ \mathrm{~N}(2)-\mathrm{C}(87)-\mathrm{H}(87 \mathrm{~B}) & 108.6 \\ \mathrm{C}(88)-\mathrm{C}(87)-\mathrm{H}(87 \mathrm{~B}) & 108.6 \\ \mathrm{H}(87 \mathrm{~A})-\mathrm{C}(87)-\mathrm{H}(87 \mathrm{~B}) & 107.6 \\ \mathrm{C}(87)-\mathrm{C}(88)-\mathrm{H}(88 \mathrm{~A}) & 109.5 \\ \mathrm{C}(87)-\mathrm{C}(88)-\mathrm{H}(88 \mathrm{~B}) & 109.5 \\ \mathrm{H}(88 \mathrm{~A})-\mathrm{C}(88)-\mathrm{H}(88 \mathrm{~B}) & 109.5 \\ \mathrm{C}(87)-\mathrm{C}(88)-\mathrm{H}(88 \mathrm{C}) & 109.5 \\ \mathrm{H}(88 \mathrm{~A})-\mathrm{C}(88)-\mathrm{H}(88 \mathrm{C}) & 109.5 \\ \mathrm{H}(88 \mathrm{~B})-\mathrm{C}(88)-\mathrm{H}(88 \mathrm{C}) & 109.5 \\ \mathrm{C}(83)-\mathrm{C}(84)-\mathrm{H}(84 \mathrm{~A}) & 109.5 \\ \mathrm{C}(83)-\mathrm{C}(84)-\mathrm{H}(84 \mathrm{~B}) & 109.5 \\ \mathrm{H}(84 \mathrm{~A})-\mathrm{C}(84)-\mathrm{H}(84 \mathrm{~B}) & 109.5 \\ \mathrm{C}(83)-\mathrm{C}(84)-\mathrm{H}(84 \mathrm{C}) & 109.5 \\ \mathrm{H}(84 \mathrm{~A})-\mathrm{C}(84)-\mathrm{H}(84 \mathrm{C}) & 109.5 \\ \mathrm{H}(84 \mathrm{~B})-\mathrm{C}(84)-\mathrm{H}(84 \mathrm{C}) & 109.5 \\ \mathrm{C}(85)-\mathrm{C}(86)-\mathrm{H}(86 \mathrm{~A}) & 109.5 \\ \mathrm{C}(85)-\mathrm{C}(86)-\mathrm{H}(86 \mathrm{~B}) & 109.5 \\ \mathrm{H}(86 \mathrm{~A})-\mathrm{C}(86)-\mathrm{H}(86 \mathrm{~B}) & 109.5 \\ \mathrm{C}(85)-\mathrm{C}(86)-\mathrm{H}(86 \mathrm{C}) & 109.5 \\ \mathrm{H}(86 \mathrm{~A})-\mathrm{C}(86)-\mathrm{H}(86 \mathrm{C}) & 109.5 \\ \mathrm{H}(86 \mathrm{~B})-\mathrm{C}(86)-\mathrm{H}(86 \mathrm{C}) & 109.5 \\ \mathrm{C}(84)-\mathrm{C}(83)-\mathrm{N}(2) & 150.6(6) \\ \mathrm{C}(84)-\mathrm{C}(83)-\mathrm{H}(83 \mathrm{~A}) & 99.0 \\ \mathrm{~N}(2)-\mathrm{C}(83)-\mathrm{H}(83 \mathrm{~A}) & 99.0 \\ \mathrm{C}(84)-\mathrm{C}(83)-\mathrm{H}(83 \mathrm{~B}) & 99.0 \\ \mathrm{~N}(2)-\mathrm{C}(83)-\mathrm{H}(83 \mathrm{~B}) & 99.0 \\ \mathrm{H}(83 \mathrm{~A})-\mathrm{C}(83)-\mathrm{H}(83 \mathrm{~B}) & 103.9 \\ \mathrm{C}(86)-\mathrm{C}(85)-\mathrm{N}(2) & 156.9(12) \\ \mathrm{C}(86)-\mathrm{C}(85)-\mathrm{H}(85 \mathrm{~A}) & 97.1 \\ \mathrm{~N}(2)-\mathrm{C}(85)-\mathrm{H}(85 \mathrm{~A}) & 97.1 \\ \mathrm{C}(86)-\mathrm{C}(85)-\mathrm{H}(85 \mathrm{~B}) & 97.1 \\ \mathrm{~N}(2)-\mathrm{C}(85)-\mathrm{H}(85 \mathrm{~B}) & 97.1 \\ \mathrm{H}(85 \mathrm{~A})-\mathrm{C}(85)-\mathrm{H}(85 \mathrm{~B}) & 103.5 \\ & \\ & \end{array}$


Table 4. Anisotropic displacement parameters $\left(\AA^{2} \times 10^{3}\right)$ for $\left\{\left[\mathrm{NEt}_{4}\right]\left[\mathrm{Ph}_{3} \mathrm{BP}^{m-\mathrm{Ph} 2}\right]\right\}_{2}$. The anisotropicdisplacement factor exponent takes the form: $-2 \pi^{2}\left[h^{2} a^{* 2} U^{11}+\ldots+2\right.$ $\mathrm{h} \mathrm{k} \mathrm{a*b*} \mathrm{U}^{12}$ ]

\begin{tabular}{|c|c|c|c|c|c|c|}
\hline & $\mathrm{U}^{11}$ & $\mathrm{U}^{22}$ & $\mathrm{U}^{33}$ & $\mathrm{U}^{23}$ & $\mathrm{U}^{13}$ & $\mathrm{U}^{12}$ \\
\hline $\mathrm{B}(1)$ & $18(2)$ & $18(2)$ & $17(2)$ & $-2(2)$ & $2(2)$ & $-2(2)$ \\
\hline $\mathrm{P}(1)$ & $37(1)$ & $24(1)$ & $29(1)$ & $-2(1)$ & $-7(1)$ & $3(1)$ \\
\hline $\mathrm{C}(1)$ & $11(2)$ & $20(2)$ & $37(2)$ & $4(2)$ & $2(2)$ & $-2(2)$ \\
\hline$C(2)$ & $32(2)$ & $29(2)$ & $62(3)$ & $10(2)$ & $-17(2)$ & $-10(2)$ \\
\hline$C(3)$ & $31(2)$ & $32(3)$ & $83(4)$ & $20(2)$ & $-15(2)$ & $-1(2)$ \\
\hline C(4) & $18(2)$ & $29(3)$ & $98(4)$ & $-3(3)$ & $2(2)$ & $2(2)$ \\
\hline$C(5)$ & $39(3)$ & $35(3)$ & $72(3)$ & $-19(2)$ & $25(2)$ & $-10(2)$ \\
\hline$C(6)$ & $40(2)$ & $22(2)$ & $46(3)$ & $-6(2)$ & $20(2)$ & $-9(2)$ \\
\hline$C(7)$ & $24(2)$ & $17(2)$ & $19(2)$ & $-6(2)$ & $-2(2)$ & $-5(2)$ \\
\hline$C(8)$ & $33(2)$ & $20(2)$ & $29(2)$ & $-2(2)$ & $4(2)$ & $-8(2)$ \\
\hline$C(9)$ & $46(2)$ & $35(2)$ & $25(2)$ & $-11(2)$ & $14(2)$ & $-18(2)$ \\
\hline$C(10)$ & $62(3)$ & $41(3)$ & $26(2)$ & $6(2)$ & $7(2)$ & $-25(2)$ \\
\hline $\mathrm{C}(11)$ & $61(3)$ & $31(2)$ & $35(2)$ & $12(2)$ & $2(2)$ & $-4(2)$ \\
\hline$C(12)$ & $54(3)$ & $26(2)$ & $29(2)$ & $2(2)$ & $9(2)$ & $3(2)$ \\
\hline$C(13)$ & $23(2)$ & $8(2)$ & $28(2)$ & $-2(2)$ & $-3(2)$ & 2(1) \\
\hline$C(14)$ & $38(2)$ & $19(2)$ & $41(2)$ & $-10(2)$ & $-7(2)$ & $1(2)$ \\
\hline$C(15)$ & $25(2)$ & $23(2)$ & $66(3)$ & $-11(2)$ & $-17(2)$ & $1(2)$ \\
\hline$C(16)$ & $22(2)$ & $19(2)$ & $87(4)$ & $4(2)$ & $5(2)$ & $-4(2)$ \\
\hline$C(17)$ & $37(3)$ & $60(3)$ & $60(3)$ & $19(3)$ & $19(2)$ & $2(2)$ \\
\hline$C(18)$ & $24(2)$ & $51(3)$ & $36(2)$ & $11(2)$ & $7(2)$ & $-4(2)$ \\
\hline$C(19)$ & $20(2)$ & $17(2)$ & $20(2)$ & $6(2)$ & $3(2)$ & $-3(2)$ \\
\hline$C(20)$ & $30(2)$ & $18(2)$ & $22(2)$ & $1(2)$ & $-1(2)$ & $-2(2)$ \\
\hline$C(21)$ & $23(2)$ & $27(2)$ & $29(2)$ & $7(2)$ & $-5(2)$ & $1(2)$ \\
\hline$C(22)$ & $28(2)$ & $32(2)$ & $22(2)$ & $2(2)$ & $-7(2)$ & $-4(2)$ \\
\hline$C(23)$ & $29(2)$ & $16(2)$ & $22(2)$ & $-1(2)$ & $-3(2)$ & $0(2)$ \\
\hline$C(24)$ & $24(2)$ & $27(2)$ & $19(2)$ & $2(2)$ & $-5(2)$ & $4(2)$ \\
\hline$C(25)$ & $30(2)$ & $26(2)$ & $31(2)$ & $-4(2)$ & $-4(2)$ & $1(2)$ \\
\hline$C(26)$ & $44(2)$ & $29(2)$ & $27(2)$ & $-4(2)$ & $-3(2)$ & $0(2)$ \\
\hline$C(27)$ & $48(3)$ & $29(2)$ & $38(2)$ & $0(2)$ & $3(2)$ & $-9(2)$ \\
\hline$C(28)$ & $35(2)$ & $28(2)$ & $44(3)$ & $-5(2)$ & 1(2) & $-4(2)$ \\
\hline$C(29)$ & $36(2)$ & $27(2)$ & $40(2)$ & $1(2)$ & $-6(2)$ & $-2(2)$ \\
\hline $\mathrm{C}(30)$ & $33(2)$ & $23(2)$ & $35(2)$ & $-1(2)$ & $-6(2)$ & $-4(2)$ \\
\hline$C(31)$ & $29(2)$ & $26(2)$ & $35(2)$ & $-12(2)$ & $-14(2)$ & $7(2)$ \\
\hline$C(32)$ & $29(2)$ & $35(2)$ & $38(2)$ & $-3(2)$ & $-2(2)$ & $-1(2)$ \\
\hline$C(33)$ & $35(2)$ & $35(2)$ & $50(3)$ & $6(2)$ & $-6(2)$ & $-5(2)$ \\
\hline$C(34)$ & $27(2)$ & $67(3)$ & $33(2)$ & $2(2)$ & $0(2)$ & $-6(2)$ \\
\hline$C(35)$ & $35(3)$ & $49(3)$ & $48(3)$ & $-22(2)$ & $-1(2)$ & $2(2)$ \\
\hline$C(36)$ & $31(2)$ & $39(3)$ & $38(2)$ & $-7(2)$ & $2(2)$ & $-1(2)$ \\
\hline $\mathrm{B}(2)$ & $23(2)$ & $18(2)$ & $11(2)$ & $0(2)$ & $4(2)$ & $-2(2)$ \\
\hline $\mathrm{P}(2)$ & $33(1)$ & $23(1)$ & $32(1)$ & $4(1)$ & $-9(1)$ & $-5(1)$ \\
\hline$C(37)$ & $23(2)$ & $10(2)$ & $22(2)$ & $3(2)$ & $2(2)$ & $0(1)$ \\
\hline$C(38)$ & $26(2)$ & $18(2)$ & $28(2)$ & $-2(2)$ & $2(2)$ & $-1(2)$ \\
\hline$C(39)$ & $24(2)$ & $20(2)$ & $38(2)$ & $1(2)$ & $12(2)$ & $3(2)$ \\
\hline $\mathrm{C}(40)$ & $18(2)$ & $18(2)$ & $49(3)$ & $6(2)$ & $-3(2)$ & $-4(2)$ \\
\hline $\mathrm{C}(41)$ & $36(2)$ & $15(2)$ & $35(2)$ & $6(2)$ & $-9(2)$ & $2(2)$ \\
\hline$C(42)$ & $23(2)$ & $21(2)$ & $24(2)$ & $4(2)$ & $2(2)$ & $3(2)$ \\
\hline
\end{tabular}




\begin{tabular}{|c|c|c|c|c|c|c|}
\hline$C(43)$ & $13(2)$ & $22(2)$ & $18(2)$ & $-2(2)$ & $5(1)$ & $-3(2)$ \\
\hline$C(44)$ & $22(2)$ & $21(2)$ & $24(2)$ & $2(2)$ & 1(2) & $-1(2)$ \\
\hline$C(45)$ & $20(2)$ & $25(2)$ & $36(2)$ & $-11(2)$ & $4(2)$ & $-3(2)$ \\
\hline C(46) & $24(2)$ & $20(2)$ & $44(2)$ & $6(2)$ & $9(2)$ & $-1(2)$ \\
\hline$C(47)$ & $26(2)$ & $34(2)$ & $34(2)$ & $15(2)$ & $14(2)$ & $7(2)$ \\
\hline C(48) & $33(2)$ & $24(2)$ & $24(2)$ & $2(2)$ & $6(2)$ & $2(2)$ \\
\hline C(49) & $23(2)$ & $20(2)$ & $14(2)$ & $4(2)$ & $-3(2)$ & $2(2)$ \\
\hline $\mathrm{C}(50)$ & $35(2)$ & $17(2)$ & $38(2)$ & $0(2)$ & $13(2)$ & $3(2)$ \\
\hline $\mathrm{C}(51)$ & 43(3) & $33(2)$ & $43(3)$ & $9(2)$ & $26(2)$ & $4(2)$ \\
\hline$C(52)$ & $47(3)$ & $38(2)$ & $18(2)$ & $-3(2)$ & $7(2)$ & $13(2)$ \\
\hline $\mathrm{C}(53)$ & $31(2)$ & $27(2)$ & $36(2)$ & $-11(2)$ & $-4(2)$ & $5(2)$ \\
\hline $\mathrm{C}(54)$ & $29(2)$ & $24(2)$ & $26(2)$ & $-2(2)$ & $3(2)$ & $3(2)$ \\
\hline$C(55)$ & $22(2)$ & $17(2)$ & $16(2)$ & $-5(2)$ & $8(2)$ & $0(2)$ \\
\hline $\mathrm{C}(56)$ & $32(2)$ & $20(2)$ & $24(2)$ & $-1(2)$ & $-2(2)$ & $-3(2)$ \\
\hline $\mathrm{C}(57)$ & $33(2)$ & $19(2)$ & $19(2)$ & $3(2)$ & $1(2)$ & $-4(2)$ \\
\hline $\mathrm{C}(58)$ & $33(2)$ & $21(2)$ & $28(2)$ & $5(2)$ & $-6(2)$ & $1(2)$ \\
\hline C(59) & $26(2)$ & $27(2)$ & $27(2)$ & 1(2) & $0(2)$ & $-6(2)$ \\
\hline $\mathrm{C}(60)$ & $23(2)$ & $19(2)$ & $24(2)$ & $3(2)$ & $2(2)$ & $-2(2)$ \\
\hline $\mathrm{C}(61)$ & $26(2)$ & $30(2)$ & $27(2)$ & $7(2)$ & $-3(2)$ & $0(2)$ \\
\hline $\mathrm{C}(62)$ & $33(2)$ & $24(2)$ & $43(2)$ & $7(2)$ & $-4(2)$ & $1(2)$ \\
\hline$C(63)$ & $36(2)$ & $43(3)$ & $41(3)$ & $20(2)$ & $2(2)$ & $1(2)$ \\
\hline $\mathrm{C}(64)$ & $33(2)$ & 44(3) & $35(2)$ & $5(2)$ & $1(2)$ & $-3(2)$ \\
\hline $\mathrm{C}(65)$ & $35(2)$ & $33(2)$ & $31(2)$ & $0(2)$ & $-3(2)$ & $-5(2)$ \\
\hline$C(66)$ & $29(2)$ & $27(2)$ & $32(2)$ & $5(2)$ & $-6(2)$ & $-7(2)$ \\
\hline$C(67)$ & $34(2)$ & $19(2)$ & $32(2)$ & $6(2)$ & $-4(2)$ & $-2(2)$ \\
\hline $\mathrm{C}(68)$ & $45(2)$ & $24(2)$ & $37(2)$ & $3(2)$ & $-2(2)$ & $4(2)$ \\
\hline $\mathrm{C}(69)$ & $45(3)$ & $33(3)$ & $62(3)$ & $-1(2)$ & $9(2)$ & $12(2)$ \\
\hline$C(70)$ & $37(3)$ & $36(3)$ & $69(3)$ & $16(2)$ & $-14(2)$ & $5(2)$ \\
\hline $\mathrm{C}(71)$ & $40(3)$ & $26(2)$ & $53(3)$ & $9(2)$ & $-16(2)$ & $-3(2)$ \\
\hline $\mathrm{C}(72)$ & $39(2)$ & $22(2)$ & $39(2)$ & $2(2)$ & $-11(2)$ & $-1(2)$ \\
\hline $\mathrm{N}(1)$ & $18(2)$ & $18(2)$ & $26(2)$ & $2(1)$ & $0(1)$ & $1(1)$ \\
\hline$C(73)$ & $30(2)$ & $24(2)$ & $30(2)$ & $4(2)$ & $-1(2)$ & $-1(2)$ \\
\hline$C(74)$ & $32(2)$ & $43(2)$ & $29(2)$ & $8(2)$ & $6(2)$ & $-4(2)$ \\
\hline$C(75)$ & $27(2)$ & $24(2)$ & $33(2)$ & $10(2)$ & $8(2)$ & $3(2)$ \\
\hline$C(76)$ & $36(2)$ & $21(2)$ & $51(3)$ & $3(2)$ & $9(2)$ & $5(2)$ \\
\hline$C(77)$ & $18(2)$ & $17(2)$ & $37(2)$ & 1(2) & $4(2)$ & $-2(2)$ \\
\hline $\mathrm{C}(78)$ & $26(2)$ & $23(2)$ & $53(3)$ & $2(2)$ & $3(2)$ & $3(2)$ \\
\hline $\mathrm{C}(79)$ & $23(2)$ & $23(2)$ & $28(2)$ & $-2(2)$ & $3(2)$ & $-1(2)$ \\
\hline $\mathrm{C}(80)$ & $39(2)$ & $39(2)$ & $31(2)$ & $2(2)$ & $4(2)$ & $-8(2)$ \\
\hline $\mathrm{N}(2)$ & $23(2)$ & $26(2)$ & $74(3)$ & $5(2)$ & $-2(2)$ & $0(1)$ \\
\hline $\mathrm{C}(81)$ & $83(4)$ & $137(6)$ & $130(6)$ & $-35(5)$ & $-62(4)$ & 21(4) \\
\hline $\mathrm{C}(82)$ & $163(6)$ & 193(7) & $82(4)$ & $-7(5)$ & $-14(4)$ & $152(6)$ \\
\hline $\mathrm{C}(87)$ & $102(5)$ & $101(6)$ & $325(12)$ & $-135(7)$ & $-21(6)$ & $51(4)$ \\
\hline $\mathrm{C}(88)$ & 203(7) & $95(5)$ & $81(4)$ & $-20(4)$ & $-84(5)$ & $71(5)$ \\
\hline $\mathrm{C}(84)$ & $37(3)$ & $83(4)$ & $188(6)$ & $70(4)$ & $-4(4)$ & $-11(3)$ \\
\hline$C(86)$ & 41(3) & $45(4)$ & 432(13) & $37(6)$ & $26(5)$ & $-25(3)$ \\
\hline$C(83)$ & $51(4)$ & $860(30)$ & $85(5)$ & $147(9)$ & $-13(4)$ & $-172(9)$ \\
\hline$C(85)$ & $830(30)$ & $15(4)$ & $134(7)$ & $14(4)$ & $217(11)$ & $45(8)$ \\
\hline
\end{tabular}


Table 5. Hydrogen coordinates $\left(\mathrm{x} 10^{4}\right)$ and isotropic displacement parameters $\left(\AA^{2} \times 10\right.$ 3 ) for $\left\{\left[\mathrm{NEt}_{4}\right]\left[\mathrm{Ph}_{3} \mathrm{BP}^{m-\mathrm{Ph} 2}\right]\right\}_{2}$.

\begin{tabular}{|c|c|c|c|c|}
\hline & $\mathrm{x}$ & $\mathrm{y}$ & $\mathrm{z}$ & $\mathrm{U}(\mathrm{eq})$ \\
\hline $\mathrm{H}(2)$ & 1371 & 6524 & 1378 & 49 \\
\hline $\mathrm{H}(3)$ & 1372 & 4276 & 1277 & 58 \\
\hline $\mathrm{H}(4)$ & 1155 & 3527 & 713 & 58 \\
\hline $\mathrm{H}(5)$ & 865 & 4880 & 257 & 58 \\
\hline $\mathrm{H}(6)$ & 896 & 7067 & 358 & 43 \\
\hline $\mathrm{H}(8)$ & 2178 & 8091 & 439 & 33 \\
\hline $\mathrm{H}(9)$ & 2682 & 9230 & -22 & 42 \\
\hline $\mathrm{H}(10)$ & 2245 & 11260 & -154 & 51 \\
\hline $\mathrm{H}(11)$ & 1258 & 12094 & 160 & 51 \\
\hline $\mathrm{H}(12)$ & 754 & 10942 & 621 & 44 \\
\hline $\mathrm{H}(14)$ & -166 & 9106 & 520 & 40 \\
\hline $\mathrm{H}(15)$ & -1430 & 9188 & 649 & 46 \\
\hline $\mathrm{H}(16)$ & -1818 & 9015 & 1222 & 52 \\
\hline $\mathrm{H}(17)$ & -958 & 8723 & 1671 & 63 \\
\hline $\mathrm{H}(18)$ & 290 & 8655 & 1543 & 44 \\
\hline $\mathrm{H}(20)$ & 2474 & 7812 & 1217 & 28 \\
\hline $\mathrm{H}(21)$ & 3278 & 8688 & 1631 & 32 \\
\hline $\mathrm{H}(22)$ & 2957 & 10541 & 1924 & 32 \\
\hline $\mathrm{H}(24)$ & 1051 & 10679 & 1368 & 28 \\
\hline $\mathrm{H}(26)$ & 2563 & 14294 & 1717 & 40 \\
\hline $\mathrm{H}(27)$ & 3640 & 15189 & 1951 & 46 \\
\hline $\mathrm{H}(28)$ & 4131 & 14431 & 2478 & 43 \\
\hline H(29) & 3562 & 12760 & 2769 & 41 \\
\hline $\mathrm{H}(30)$ & 2510 & 11814 & 2531 & 36 \\
\hline $\mathrm{H}(32)$ & 1411 & 9715 & 2224 & 40 \\
\hline $\mathrm{H}(33)$ & 748 & 8974 & 2699 & 48 \\
\hline $\mathrm{H}(34)$ & 81 & 10389 & 3051 & 51 \\
\hline $\mathrm{H}(35)$ & 54 & 12552 & 2899 & 52 \\
\hline $\mathrm{H}(36)$ & 698 & 13282 & 2416 & 43 \\
\hline $\mathrm{H}(38)$ & 4929 & 6193 & 1417 & 29 \\
\hline H(39) & 3645 & 6065 & 1326 & 33 \\
\hline $\mathrm{H}(40)$ & 3174 & 5734 & 762 & 34 \\
\hline $\mathrm{H}(41)$ & 3976 & 5496 & 290 & 35 \\
\hline $\mathrm{H}(42)$ & 5246 & 5613 & 382 & 27 \\
\hline $\mathrm{H}(44)$ & 6168 & 8282 & 1445 & 27 \\
\hline $\mathrm{H}(45)$ & 6242 & 10487 & 1384 & 32 \\
\hline $\mathrm{H}(46)$ & 6289 & 11407 & 826 & 35 \\
\hline $\mathrm{H}(47)$ & 6247 & 10100 & 332 & 37 \\
\hline $\mathrm{H}(48)$ & 6165 & 7920 & 395 & 33 \\
\hline $\mathrm{H}(50)$ & 7346 & 6942 & 528 & 36 \\
\hline $\mathrm{H}(51)$ & 7978 & 5880 & 91 & 47 \\
\hline $\mathrm{H}(52)$ & 7666 & 3791 & -60 & 41 \\
\hline $\mathrm{H}(53)$ & 6755 & 2751 & 262 & 38 \\
\hline $\mathrm{H}(54)$ & 6107 & 3822 & 688 & 31 \\
\hline $\mathrm{H}(56)$ & 5815 & 4324 & 1457 & 30 \\
\hline $\mathrm{H}(58)$ & 7564 & 4543 & 2127 & 33 \\
\hline $\mathrm{H}(59)$ & 8011 & 6277 & 1808 & 32 \\
\hline $\mathrm{H}(60)$ & 7373 & 7012 & 1321 & 27 \\
\hline
\end{tabular}




\begin{tabular}{|c|c|c|c|c|}
\hline $\mathrm{H}(62)$ & 5538 & 1871 & 2651 & 40 \\
\hline $\mathrm{H}(63)$ & 4970 & 2669 & 3146 & 48 \\
\hline $\mathrm{H}(64)$ & 4908 & 4858 & 3242 & 45 \\
\hline $\mathrm{H}(65)$ & 5442 & 6243 & 2840 & 40 \\
\hline $\mathrm{H}(66)$ & 5993 & 5462 & 2336 & 35 \\
\hline $\mathrm{H}(68)$ & 7159 & 728 & 1894 & 43 \\
\hline $\mathrm{H}(69)$ & 8217 & -228 & 2127 & 56 \\
\hline $\mathrm{H}(70)$ & 8766 & 580 & 2641 & 57 \\
\hline $\mathrm{H}(71)$ & 8241 & 2333 & 2916 & 48 \\
\hline $\mathrm{H}(72)$ & 7213 & 3311 & 2677 & 40 \\
\hline $\mathrm{H}(73 \mathrm{~A})$ & 3775 & 2770 & 386 & 33 \\
\hline $\mathrm{H}(73 \mathrm{~B})$ & 3711 & 1316 & 266 & 33 \\
\hline $\mathrm{H}(74 \mathrm{~A})$ & 5072 & 2605 & 400 & 52 \\
\hline $\mathrm{H}(74 \mathrm{~B})$ & 4762 & 2219 & 19 & 52 \\
\hline $\mathrm{H}(74 \mathrm{C})$ & 5016 & 1135 & 290 & 52 \\
\hline $\mathrm{H}(75 \mathrm{~A})$ & 4847 & 317 & 834 & 34 \\
\hline $\mathrm{H}(75 \mathrm{~B})$ & 4211 & -21 & 1108 & 34 \\
\hline $\mathrm{H}(76 \mathrm{~A})$ & 3511 & -959 & 649 & 54 \\
\hline $\mathrm{H}(76 \mathrm{~B})$ & 4299 & -1641 & 695 & 54 \\
\hline $\mathrm{H}(76 \mathrm{C})$ & 4165 & -650 & 382 & 54 \\
\hline $\mathrm{H}(77 \mathrm{~A})$ & 3089 & 1018 & 1119 & 29 \\
\hline $\mathrm{H}(77 \mathrm{~B})$ & 2886 & 831 & 714 & 29 \\
\hline $\mathrm{H}(78 \mathrm{~A})$ & 2931 & 3245 & 1057 & 51 \\
\hline $\mathrm{H}(78 \mathrm{~B})$ & 2188 & 2501 & 951 & 51 \\
\hline $\mathrm{H}(78 \mathrm{C})$ & 2719 & 3048 & 652 & 51 \\
\hline $\mathrm{H}(79 \mathrm{~A})$ & 4872 & 2477 & 958 & 29 \\
\hline $\mathrm{H}(79 \mathrm{~B})$ & 4150 & 3353 & 948 & 29 \\
\hline $\mathrm{H}(80 \mathrm{~A})$ & 3736 & 2386 & 1467 & 54 \\
\hline $\mathrm{H}(80 \mathrm{~B})$ & 4530 & 3033 & 1526 & 54 \\
\hline $\mathrm{H}(80 \mathrm{C})$ & 4466 & 1522 & 1477 & 54 \\
\hline $\mathrm{H}(81 \mathrm{~A})$ & 9537 & 3950 & 1436 & 140 \\
\hline $\mathrm{H}(81 \mathrm{~B})$ & 8754 & 3477 & 1564 & 140 \\
\hline $\mathrm{H}(82 \mathrm{~A})$ & 9132 & 1868 & 1737 & 219 \\
\hline $\mathrm{H}(82 \mathrm{~B})$ & 9891 & 2657 & 1746 & 219 \\
\hline $\mathrm{H}(82 \mathrm{C})$ & 9720 & 1702 & 1428 & 219 \\
\hline $\mathrm{H}(87 \mathrm{~A})$ & 9402 & 1654 & 914 & 211 \\
\hline $\mathrm{H}(87 \mathrm{~B})$ & 9900 & 2897 & 860 & 211 \\
\hline $\mathrm{H}(88 \mathrm{~A})$ & 8967 & 1734 & 373 & 190 \\
\hline $\mathrm{H}(88 \mathrm{~B})$ & 9634 & 2700 & 299 & 190 \\
\hline $\mathrm{H}(88 \mathrm{C})$ & 8830 & 3239 & 391 & 190 \\
\hline $\mathrm{H}(84 \mathrm{~A})$ & 7814 & 1947 & 1423 & 154 \\
\hline $\mathrm{H}(84 \mathrm{~B})$ & 7320 & 1971 & 1071 & 154 \\
\hline $\mathrm{H}(84 \mathrm{C})$ & 7377 & 3215 & 1313 & 154 \\
\hline $\mathrm{H}(86 \mathrm{~A})$ & 9198 & 5950 & 1155 & 259 \\
\hline $\mathrm{H}(86 \mathrm{~B})$ & 8827 & 6093 & 773 & 259 \\
\hline $\mathrm{H}(86 \mathrm{C})$ & 9653 & 5554 & 812 & 259 \\
\hline $\mathrm{H}(83 \mathrm{~A})$ & 7931 & 3498 & 919 & 400 \\
\hline $\mathrm{H}(83 \mathrm{~B})$ & 8215 & 2097 & 897 & 400 \\
\hline $\mathrm{H}(85 \mathrm{~A})$ & 8881 & 4357 & 685 & 391 \\
\hline $\mathrm{H}(85 \mathrm{~B})$ & 8414 & 4756 & 1008 & 391 \\
\hline
\end{tabular}


Table 6. Crystal data and structure refinement for $\left\{\left[\mathrm{NEt}_{4}\right]\left[\mathrm{Ph}_{3} \mathrm{BP}^{m-\mathrm{iPr} 2}\right]\right\}_{2}$.

Empirical formula

Formula weight

Crystal Habit

Crystal Color

Crystal size

Type of diffractometer

Wavelength

Data collection temperature

Unit cell dimensions

Volume

Z

Crystal system

Space group

Density (calculated)

$\mathrm{F}(000)$

$\theta$ range for data collection

Completeness to $\theta=28.60^{\circ}$

Index ranges

Reflections collected

Independent reflections

Absorption coefficient

Absorption correction
$\mathrm{C}_{38} \mathrm{H}_{53} \mathrm{BNP}$

565.59

acicular

colorless

$0.474 \times 0.18 \times 0.081 \mathrm{~mm}^{3}$

\section{Data Collection}

CCD area detector

$0.71073 \AA$

98(2) K

$\mathrm{a}=9.8900(8) \AA$

$\alpha=90^{\circ}$

$\mathrm{b}=20.1253(16) \AA$

$\mathrm{c}=16.7970(14) \AA$

$\beta=92.835(2)^{\circ}$

$\gamma=90^{\circ}$

3339.2(5) $\AA^{3}$

4

Monoclinic

P2(1)

$1.125 \mathrm{Mg} / \mathrm{m}^{3}$

1232

1.58 to $28.60^{\circ}$

$94.5 \%$

$-13 \leq \mathrm{h} \leq 13,-27 \leq \mathrm{k} \leq 26,-21 \leq 1 \leq 22$

50901

$15665[\mathrm{R}(\mathrm{int})=0.0696]$

$0.109 \mathrm{~mm}^{-1}$

None

\section{Structure solution and refinement}

Structure solution program

Primary solution method

Secondary solution method

Hydrogen placement

Structure refinement program
SHELXS-97 (Sheldrick, 1990)

direct methods

Difference Fourier Map

calculated positions

SHELXL-97 (Sheldrick, 1997) 
Refinement method

Data / restraints / parameters

Goodness-of-fit on $\mathrm{F}^{2}$

Final $\mathrm{R}$ indices $[\mathrm{I}>2 \operatorname{sigma}(\mathrm{I})]$

$\mathrm{R}$ indices (all data)

Absolute structure parameter

Largest diff. peak and hole
Full-matrix least-squares on $\mathrm{F}^{2}$

$15665 / 1 / 755$

1.614

$\mathrm{R} 1=0.0659, \mathrm{wR} 2=0.0917$

$\mathrm{R} 1=0.1066, \mathrm{wR} 2=0.0973$

$0.48(8)$

0.725 and -0.468 e. $\AA^{-3}$ 
Table 7. Atomic coordinates $\left(\times 10^{4}\right)$ and equivalent isotropic displacement parameters $\left(\AA^{2} \times 10^{3}\right)$ for $\left\{\left[\mathrm{NEt}_{4}\right]\left[\mathrm{Ph}_{3} \mathrm{BP}^{m-\mathrm{Pr} 2}\right]\right\}_{2}$. U(eq) is defined as one third of the trace of the orthogonalized $\mathrm{Uij}^{\mathrm{ij}}$ tensor.

\begin{tabular}{|c|c|c|c|c|}
\hline & $\mathrm{x}$ & $\mathrm{y}$ & $\mathrm{z}$ & $\mathrm{U}(\mathrm{eq})$ \\
\hline$\overline{\mathrm{B}(1)}$ & 174(3) & $7420(2)$ & $2274(2)$ & $23(1)$ \\
\hline $\mathrm{P}(1)$ & $524(1)$ & $10077(1)$ & 2851(1) & $29(1)$ \\
\hline$C(1)$ & $278(3)$ & $7186(2)$ & $3221(2)$ & $21(1)$ \\
\hline$C(2)$ & $453(3)$ & $7627(2)$ & $3852(2)$ & $24(1)$ \\
\hline$C(3)$ & $635(3)$ & $7437(2)$ & $4646(2)$ & $28(1)$ \\
\hline C(4) & $640(3)$ & 6774(2) & $4844(2)$ & $36(1)$ \\
\hline $\mathrm{C}(5)$ & $457(3)$ & $6315(2)$ & $4247(2)$ & $37(1)$ \\
\hline$C(6)$ & 289(3) & $6520(2)$ & $3457(2)$ & $31(1)$ \\
\hline$C(7)$ & 1708(3) & $7415(2)$ & $1963(2)$ & $24(1)$ \\
\hline$C(8)$ & 2529(3) & 6854(2) & 2079(2) & $32(1)$ \\
\hline $\mathrm{C}(9)$ & $3843(3)$ & $6827(2)$ & $1853(2)$ & $37(1)$ \\
\hline$C(10)$ & $4420(3)$ & $7369(2)$ & $1482(2)$ & $37(1)$ \\
\hline$C(11)$ & $3639(3)$ & $7929(2)$ & $1350(2)$ & $37(1)$ \\
\hline$C(12)$ & 2317(3) & 7940(2) & 1582(2) & $31(1)$ \\
\hline $\mathrm{C}(13)$ & $-759(3)$ & $6888(2)$ & $1739(2)$ & 19(1) \\
\hline$C(14)$ & $-565(3)$ & 6793(2) & $936(2)$ & $29(1)$ \\
\hline$C(15)$ & $-1442(3)$ & 6393(2) & $441(2)$ & $33(1)$ \\
\hline$C(16)$ & $-2503(3)$ & $6080(2)$ & $768(2)$ & $33(1)$ \\
\hline $\mathrm{C}(17)$ & $-2741(3)$ & $6153(2)$ & $1563(2)$ & $27(1)$ \\
\hline$C(18)$ & $-1882(3)$ & $6565(2)$ & $2029(2)$ & $26(1)$ \\
\hline$C(19)$ & $-537(3)$ & 8163(1) & 2194(2) & $18(1)$ \\
\hline $\mathrm{C}(20)$ & $95(3)$ & $8748(2)$ & $2460(2)$ & 21(1) \\
\hline$C(21)$ & $-493(3)$ & $9383(2)$ & $2445(2)$ & $20(1)$ \\
\hline$C(22)$ & $-1797(3)$ & $9427(2)$ & $2102(2)$ & $28(1)$ \\
\hline$C(23)$ & $-2458(3)$ & $8872(2)$ & 1819(2) & $35(1)$ \\
\hline$C(24)$ & $-1838(3)$ & $8249(2)$ & $1870(2)$ & $31(1)$ \\
\hline$C(25)$ & $-55(3)$ & $10134(2)$ & $3885(2)$ & $31(1)$ \\
\hline$C(26)$ & $487(3)$ & $9537(2)$ & $4361(2)$ & $38(1)$ \\
\hline$C(27)$ & $-1556(3)$ & 10212(2) & $3972(2)$ & $33(1)$ \\
\hline $\mathrm{C}(28)$ & $-283(3)$ & $10825(2)$ & $2385(2)$ & $29(1)$ \\
\hline$C(29)$ & $292(3)$ & $11447(2)$ & $2777(2)$ & $37(1)$ \\
\hline$C(30)$ & 17(3) & $10835(2)$ & $1497(2)$ & $38(1)$ \\
\hline $\mathrm{B}(2)$ & 4396(3) & $3909(2)$ & $2780(2)$ & $22(1)$ \\
\hline $\mathrm{P}(2)$ & 4289(1) & 1304(1) & 1983(1) & $26(1)$ \\
\hline$C(31)$ & 4283(3) & 4174(2) & $1837(2)$ & $21(1)$ \\
\hline$C(32)$ & 4483(3) & $3779(2)$ & $1176(2)$ & $31(1)$ \\
\hline$C(33)$ & 4305(3) & $4000(2)$ & $400(2)$ & $38(1)$ \\
\hline$C(34)$ & $3966(3)$ & $4652(2)$ & $245(2)$ & $40(1)$ \\
\hline$C(35)$ & 3792(3) & $5068(2)$ & $888(2)$ & $37(1)$ \\
\hline$C(36)$ & 3953(3) & 4833(2) & $1652(2)$ & $32(1)$ \\
\hline$C(37)$ & $5303(3)$ & $4442(2)$ & $3305(2)$ & $21(1)$ \\
\hline $\mathrm{C}(38)$ & $6405(3)$ & $4780(2)$ & 2993(2) & $25(1)$ \\
\hline$C(39)$ & $7286(3)$ & $5173(2)$ & $3444(2)$ & $32(1)$ \\
\hline $\mathrm{C}(40)$ & 7114(3) & $5249(2)$ & $4242(2)$ & $33(1)$ \\
\hline $\mathrm{C}(41)$ & 6051(3) & 4932(2) & $4574(2)$ & $39(1)$ \\
\hline$C(42)$ & $5172(3)$ & $4540(2)$ & $4115(2)$ & $28(1)$ \\
\hline$C(43)$ & $2838(3)$ & $3887(2)$ & $3091(2)$ & 21(1) \\
\hline
\end{tabular}




\begin{tabular}{|c|c|c|c|c|}
\hline$C(44)$ & 2121(3) & $4477(2)$ & $3211(2)$ & $34(1)$ \\
\hline$C(45)$ & $790(3)$ & $4480(2)$ & $3424(2)$ & $39(1)$ \\
\hline$C(46)$ & 101(3) & $3897(2)$ & $3539(2)$ & $32(1)$ \\
\hline$C(47)$ & $762(3)$ & $3310(2)$ & $3445(2)$ & $24(1)$ \\
\hline$C(48)$ & 2106(3) & $3305(2)$ & $3228(2)$ & $22(1)$ \\
\hline C(49) & 5133(3) & $3180(2)$ & 2853(2) & $19(1)$ \\
\hline $\mathrm{C}(50)$ & 4614(3) & $2608(1)$ & $2479(2)$ & $18(1)$ \\
\hline$C(51)$ & 5197(3) & $1983(2)$ & $2523(2)$ & $22(1)$ \\
\hline$C(52)$ & 6401(3) & 1912(2) & $2974(2)$ & $21(1)$ \\
\hline $\mathrm{C}(53)$ & 6955(3) & $2458(2)$ & $3372(2)$ & $24(1)$ \\
\hline$C(54)$ & 6331(3) & $3066(2)$ & $3314(2)$ & $20(1)$ \\
\hline$C(55)$ & $5129(3)$ & $1298(2)$ & $1013(2)$ & $28(1)$ \\
\hline$C(56)$ & $4776(3)$ & 1941(2) & $549(2)$ & $38(1)$ \\
\hline $\mathrm{C}(57)$ & 6644(3) & $1190(2)$ & $1046(2)$ & $34(1)$ \\
\hline $\mathrm{C}(58)$ & 4984(3) & $545(2)$ & $2461(2)$ & $28(1)$ \\
\hline$C(59)$ & 4453(3) & $-70(2)$ & 1993(2) & $39(1)$ \\
\hline$C(60)$ & 4603(3) & 492(2) & $3332(2)$ & $34(1)$ \\
\hline $\mathrm{N}(1)$ & $4428(2)$ & 2497(1) & $5642(2)$ & $29(1)$ \\
\hline$C(61)$ & $9200(4)$ & $4527(2)$ & $1290(2)$ & $50(1)$ \\
\hline$C(62)$ & $10218(3)$ & $5068(2)$ & $1328(2)$ & $60(1)$ \\
\hline$C(63)$ & 9668(4) & $4109(2)$ & $-68(2)$ & $49(1)$ \\
\hline $\mathrm{C}(64)$ & 8533(4) & $4497(2)$ & $-434(2)$ & $54(1)$ \\
\hline $\mathrm{C}(65)$ & $10804(4)$ & $3619(2)$ & $1137(2)$ & $54(1)$ \\
\hline$C(66)$ & $11180(3)$ & $2946(2)$ & 783(2) & $50(1)$ \\
\hline $\mathrm{C}(67)$ & $8351(4)$ & $3441(2)$ & $838(2)$ & $48(1)$ \\
\hline $\mathrm{C}(68)$ & $8177(4)$ & $3133(2)$ & $1623(2)$ & $66(1)$ \\
\hline $\mathrm{N}(2)$ & $9502(2)$ & 3903(1) & $809(2)$ & $25(1)$ \\
\hline$C(69)$ & $5598(3)$ & $2986(2)$ & $5667(2)$ & $38(1)$ \\
\hline$C(70)$ & 6036(4) & $3231(2)$ & $6479(2)$ & $57(1)$ \\
\hline$C(71)$ & $3234(3)$ & $2796(2)$ & $6028(2)$ & $43(1)$ \\
\hline$C(72)$ & 2731(3) & $3470(2)$ & $5711(2)$ & $58(1)$ \\
\hline$C(73)$ & $4759(3)$ & $1868(2)$ & $6085(2)$ & $40(1)$ \\
\hline$C(74)$ & $5921(3)$ & $1480(2)$ & $5795(2)$ & $67(1)$ \\
\hline$C(75)$ & $4095(3)$ & $2369(2)$ & $4761(2)$ & $38(1)$ \\
\hline$C(76)$ & $3003(3)$ & $1888(2)$ & $4554(2)$ & $42(1)$ \\
\hline
\end{tabular}


Table 8. Bond lengths $[\AA]$ and angles $\left[{ }^{\circ}\right]$ for $\left\{\left[\mathrm{NEt}_{4}\right]\left[\mathrm{Ph}_{3} \mathrm{BP}^{m-\mathrm{Pr} 2}\right]\right\}_{2}$.

\begin{tabular}{|c|c|c|c|}
\hline $\mathrm{B}(1)-\mathrm{C}(7)$ & $1.628(4)$ & $\mathrm{C}(25)-\mathrm{H}(25)$ & 1.0000 \\
\hline $\mathrm{B}(1)-\mathrm{C}(13)$ & $1.650(5)$ & $\mathrm{C}(26)-\mathrm{H}(26 \mathrm{~A})$ & 0.9800 \\
\hline B(1)-C(19) & $1.654(4)$ & $\mathrm{C}(26)-\mathrm{H}(26 \mathrm{~B})$ & 0.9800 \\
\hline $\mathrm{B}(1)-\mathrm{C}(1)$ & $1.657(4)$ & $\mathrm{C}(26)-\mathrm{H}(26 \mathrm{C})$ & 0.9800 \\
\hline$P(1)-C(21)$ & $1.833(3)$ & $\mathrm{C}(27)-\mathrm{H}(27 \mathrm{~A})$ & 0.9800 \\
\hline $\mathrm{P}(1)-\mathrm{C}(25)$ & $1.859(3)$ & $\mathrm{C}(27)-\mathrm{H}(27 \mathrm{~B})$ & 0.9800 \\
\hline$P(1)-C(28)$ & $1.859(3)$ & $\mathrm{C}(27)-\mathrm{H}(27 \mathrm{C})$ & 0.9800 \\
\hline $\mathrm{C}(1)-\mathrm{C}(2)$ & $1.387(4)$ & $\mathrm{C}(28)-\mathrm{C}(29)$ & $1.512(4)$ \\
\hline $\mathrm{C}(1)-\mathrm{C}(6)$ & $1.397(4)$ & $\mathrm{C}(28)-\mathrm{C}(30)$ & $1.534(4)$ \\
\hline $\mathrm{C}(2)-\mathrm{C}(3)$ & $1.390(4)$ & $\mathrm{C}(28)-\mathrm{H}(28)$ & 1.0000 \\
\hline $\mathrm{C}(2)-\mathrm{H}(2)$ & 0.9500 & $\mathrm{C}(29)-\mathrm{H}(29 \mathrm{~A})$ & 0.9800 \\
\hline $\mathrm{C}(3)-\mathrm{C}(4)$ & $1.376(4)$ & $\mathrm{C}(29)-\mathrm{H}(29 \mathrm{~B})$ & 0.9800 \\
\hline $\mathrm{C}(3)-\mathrm{H}(3)$ & 0.9500 & $\mathrm{C}(29)-\mathrm{H}(29 \mathrm{C})$ & 0.9800 \\
\hline$C(4)-C(5)$ & $1.368(4)$ & $\mathrm{C}(30)-\mathrm{H}(30 \mathrm{~A})$ & 0.9800 \\
\hline $\mathrm{C}(4)-\mathrm{H}(4)$ & 0.9500 & $\mathrm{C}(30)-\mathrm{H}(30 \mathrm{~B})$ & 0.9800 \\
\hline$C(5)-C(6)$ & $1.392(4)$ & $\mathrm{C}(30)-\mathrm{H}(30 \mathrm{C})$ & 0.9800 \\
\hline $\mathrm{C}(5)-\mathrm{H}(5)$ & 0.9500 & $\mathrm{~B}(2)-\mathrm{C}(37)$ & $1.629(4)$ \\
\hline $\mathrm{C}(6)-\mathrm{H}(6)$ & 0.9500 & $\mathrm{~B}(2)-\mathrm{C}(49)$ & $1.640(5)$ \\
\hline$C(7)-C(12)$ & $1.388(4)$ & $B(2)-C(43)$ & $1.653(4)$ \\
\hline $\mathrm{C}(7)-\mathrm{C}(8)$ & $1.400(4)$ & $\mathrm{B}(2)-\mathrm{C}(31)$ & $1.669(5)$ \\
\hline $\mathrm{C}(8)-\mathrm{C}(9)$ & $1.373(4)$ & $\mathrm{P}(2)-\mathrm{C}(58)$ & $1.842(3)$ \\
\hline $\mathrm{C}(8)-\mathrm{H}(8)$ & 0.9500 & $\mathrm{P}(2)-\mathrm{C}(51)$ & $1.849(3)$ \\
\hline$C(9)-C(10)$ & $1.392(5)$ & $\mathrm{P}(2)-\mathrm{C}(55)$ & $1.865(3)$ \\
\hline $\mathrm{C}(9)-\mathrm{H}(9)$ & 0.9500 & $\mathrm{C}(31)-\mathrm{C}(32)$ & $1.388(4)$ \\
\hline $\mathrm{C}(10)-\mathrm{C}(11)$ & $1.379(4)$ & $C(31)-C(36)$ & $1.397(4)$ \\
\hline C(10)-H(10) & 0.9500 & $C(32)-C(33)$ & $1.380(4)$ \\
\hline C(11)-C(12) & $1.383(4)$ & $\mathrm{C}(32)-\mathrm{H}(32)$ & 0.9500 \\
\hline C(11)-H(11) & 0.9500 & $\mathrm{C}(33)-\mathrm{C}(34)$ & $1.376(5)$ \\
\hline C(12)-H(12) & 0.9500 & $\mathrm{C}(33)-\mathrm{H}(33)$ & 0.9500 \\
\hline C(13)-C(14) & $1.385(4)$ & $C(34)-C(35)$ & $1.384(4)$ \\
\hline C(13)-C(18) & $1.395(4)$ & $\mathrm{C}(34)-\mathrm{H}(34)$ & 0.9500 \\
\hline$C(14)-C(15)$ & $1.421(4)$ & $C(35)-C(36)$ & $1.370(4)$ \\
\hline $\mathrm{C}(14)-\mathrm{H}(14)$ & 0.9500 & $\mathrm{C}(35)-\mathrm{H}(35)$ & 0.9500 \\
\hline C(15)-C(16) & $1.363(4)$ & $\mathrm{C}(36)-\mathrm{H}(36)$ & 0.9500 \\
\hline C(15)-H(15) & 0.9500 & $C(37)-C(42)$ & $1.388(4)$ \\
\hline C(16)-C(17) & $1.374(4)$ & $\mathrm{C}(37)-\mathrm{C}(38)$ & $1.408(4)$ \\
\hline C(16)-H(16) & 0.9500 & $\mathrm{C}(38)-\mathrm{C}(39)$ & $1.375(4)$ \\
\hline C(17)-C(18) & $1.400(4)$ & $\mathrm{C}(38)-\mathrm{H}(38)$ & 0.9500 \\
\hline $\mathrm{C}(17)-\mathrm{H}(17)$ & 0.9500 & $\mathrm{C}(39)-\mathrm{C}(40)$ & $1.367(4)$ \\
\hline C(18)-H(18) & 0.9500 & $\mathrm{C}(39)-\mathrm{H}(39)$ & 0.9500 \\
\hline C(19)-C(24) & $1.384(4)$ & $\mathrm{C}(40)-\mathrm{C}(41)$ & $1.372(4)$ \\
\hline C(19)-C(20) & $1.396(4)$ & $\mathrm{C}(40)-\mathrm{H}(40)$ & 0.9500 \\
\hline$C(20)-C(21)$ & $1.403(4)$ & $\mathrm{C}(41)-\mathrm{C}(42)$ & $1.380(4)$ \\
\hline $\mathrm{C}(20)-\mathrm{H}(20)$ & 0.9500 & $\mathrm{C}(41)-\mathrm{H}(41)$ & 0.9500 \\
\hline C(21)-C(22) & $1.389(4)$ & $\mathrm{C}(42)-\mathrm{H}(42)$ & 0.9500 \\
\hline $\mathrm{C}(22)-\mathrm{C}(23)$ & $1.368(4)$ & $C(43)-C(48)$ & $1.401(4)$ \\
\hline C(22)-H(22) & 0.9500 & C(43)-C(44) & $1.403(4)$ \\
\hline $\mathrm{C}(23)-\mathrm{C}(24)$ & $1.396(4)$ & $\mathrm{C}(44)-\mathrm{C}(45)$ & $1.380(4)$ \\
\hline $\mathrm{C}(23)-\mathrm{H}(23)$ & 0.9500 & $\mathrm{C}(44)-\mathrm{H}(44)$ & 0.9500 \\
\hline C(24)-H(24) & 0.9500 & $C(45)-C(46)$ & $1.375(4)$ \\
\hline$C(25)-C(27)$ & $1.507(4)$ & $\mathrm{C}(45)-\mathrm{H}(45)$ & 0.9500 \\
\hline$C(25)-C(26)$ & $1.525(4)$ & $C(46)-C(47)$ & $1.364(4)$ \\
\hline
\end{tabular}




\begin{tabular}{|c|c|c|c|}
\hline $\mathrm{C}(46)-\mathrm{H}(46)$ & 0.9500 & $\mathrm{C}(66)-\mathrm{H}(66 \mathrm{C})$ & 0.9800 \\
\hline $\mathrm{C}(47)-\mathrm{C}(48)$ & $1.396(4)$ & $\mathrm{C}(67)-\mathrm{N}(2)$ & $1.473(4)$ \\
\hline $\mathrm{C}(47)-\mathrm{H}(47)$ & 0.9500 & $C(67)-C(68)$ & $1.474(5)$ \\
\hline $\mathrm{C}(48)-\mathrm{H}(48)$ & 0.9500 & $\mathrm{C}(67)-\mathrm{H}(67 \mathrm{~A})$ & 0.9900 \\
\hline $\mathrm{C}(49)-\mathrm{C}(50)$ & $1.397(4)$ & $\mathrm{C}(67)-\mathrm{H}(67 \mathrm{~B})$ & 0.9900 \\
\hline$C(49)-C(54)$ & $1.401(4)$ & $\mathrm{C}(68)-\mathrm{H}(68 \mathrm{~A})$ & 0.9800 \\
\hline$C(50)-C(51)$ & $1.383(4)$ & $\mathrm{C}(68)-\mathrm{H}(68 \mathrm{~B})$ & 0.9800 \\
\hline $\mathrm{C}(50)-\mathrm{H}(50)$ & 0.9500 & $\mathrm{C}(68)-\mathrm{H}(68 \mathrm{C})$ & 0.9800 \\
\hline $\mathrm{C}(51)-\mathrm{C}(52)$ & $1.387(4)$ & $\mathrm{C}(69)-\mathrm{C}(70)$ & $1.493(4)$ \\
\hline$C(52)-C(53)$ & $1.385(4)$ & $\mathrm{C}(69)-\mathrm{H}(69 \mathrm{~A})$ & 0.9900 \\
\hline $\mathrm{C}(52)-\mathrm{H}(52)$ & 0.9500 & $\mathrm{C}(69)-\mathrm{H}(69 \mathrm{~B})$ & 0.9900 \\
\hline $\mathrm{C}(53)-\mathrm{C}(54)$ & $1.372(4)$ & $\mathrm{C}(70)-\mathrm{H}(70 \mathrm{~A})$ & 0.9800 \\
\hline $\mathrm{C}(53)-\mathrm{H}(53)$ & 0.9500 & $\mathrm{C}(70)-\mathrm{H}(70 \mathrm{~B})$ & 0.9800 \\
\hline $\mathrm{C}(54)-\mathrm{H}(54)$ & 0.9500 & $\mathrm{C}(70)-\mathrm{H}(70 \mathrm{C})$ & 0.9800 \\
\hline $\mathrm{C}(55)-\mathrm{C}(57)$ & $1.512(4)$ & $\mathrm{C}(71)-\mathrm{C}(72)$ & $1.530(4)$ \\
\hline $\mathrm{C}(55)-\mathrm{C}(56)$ & $1.542(4)$ & $\mathrm{C}(71)-\mathrm{H}(71 \mathrm{~A})$ & 0.9900 \\
\hline $\mathrm{C}(55)-\mathrm{H}(55)$ & 1.0000 & $\mathrm{C}(71)-\mathrm{H}(71 \mathrm{~B})$ & 0.9900 \\
\hline $\mathrm{C}(56)-\mathrm{H}(56 \mathrm{~A})$ & 0.9800 & $\mathrm{C}(72)-\mathrm{H}(72 \mathrm{~A})$ & 0.9800 \\
\hline $\mathrm{C}(56)-\mathrm{H}(56 \mathrm{~B})$ & 0.9800 & $\mathrm{C}(72)-\mathrm{H}(72 \mathrm{~B})$ & 0.9800 \\
\hline $\mathrm{C}(56)-\mathrm{H}(56 \mathrm{C})$ & 0.9800 & $\mathrm{C}(72)-\mathrm{H}(72 \mathrm{C})$ & 0.9800 \\
\hline $\mathrm{C}(57)-\mathrm{H}(57 \mathrm{~A})$ & 0.9800 & $\mathrm{C}(73)-\mathrm{C}(74)$ & $1.491(4)$ \\
\hline $\mathrm{C}(57)-\mathrm{H}(57 \mathrm{~B})$ & 0.9800 & $\mathrm{C}(73)-\mathrm{H}(73 \mathrm{~A})$ & 0.9900 \\
\hline $\mathrm{C}(57)-\mathrm{H}(57 \mathrm{C})$ & 0.9800 & $\mathrm{C}(73)-\mathrm{H}(73 \mathrm{~B})$ & 0.9900 \\
\hline $\mathrm{C}(58)-\mathrm{C}(60)$ & $1.533(4)$ & $\mathrm{C}(74)-\mathrm{H}(74 \mathrm{~A})$ & 0.9800 \\
\hline $\mathrm{C}(58)-\mathrm{C}(59)$ & $1.546(4)$ & $\mathrm{C}(74)-\mathrm{H}(74 \mathrm{~B})$ & 0.9800 \\
\hline $\mathrm{C}(58)-\mathrm{H}(58)$ & 1.0000 & $\mathrm{C}(74)-\mathrm{H}(74 \mathrm{C})$ & 0.9800 \\
\hline $\mathrm{C}(59)-\mathrm{H}(59 \mathrm{~A})$ & 0.9800 & $C(75)-C(76)$ & $1.480(4)$ \\
\hline $\mathrm{C}(59)-\mathrm{H}(59 \mathrm{~B})$ & 0.9800 & $\mathrm{C}(75)-\mathrm{H}(75 \mathrm{~A})$ & 0.9900 \\
\hline $\mathrm{C}(59)-\mathrm{H}(59 \mathrm{C})$ & 0.9800 & $\mathrm{C}(75)-\mathrm{H}(75 \mathrm{~B})$ & 0.9900 \\
\hline $\mathrm{C}(60)-\mathrm{H}(60 \mathrm{~A})$ & 0.9800 & $\mathrm{C}(76)-\mathrm{H}(76 \mathrm{~A})$ & 0.9800 \\
\hline $\mathrm{C}(60)-\mathrm{H}(60 \mathrm{~B})$ & 0.9800 & $\mathrm{C}(76)-\mathrm{H}(76 \mathrm{~B})$ & 0.9800 \\
\hline $\mathrm{C}(60)-\mathrm{H}(60 \mathrm{C})$ & 0.9800 & $\mathrm{C}(76)-\mathrm{H}(76 \mathrm{C})$ & 0.9800 \\
\hline $\mathrm{N}(1)-\mathrm{C}(73)$ & $1.496(4)$ & & \\
\hline $\mathrm{N}(1)-\mathrm{C}(71)$ & $1.501(4)$ & $\mathrm{C}(7)-\mathrm{B}(1)-\mathrm{C}(13)$ & 109.0(3) \\
\hline $\mathrm{N}(1)-\mathrm{C}(69)$ & $1.517(4)$ & $\mathrm{C}(7)-\mathrm{B}(1)-\mathrm{C}(19)$ & $112.3(3)$ \\
\hline $\mathrm{N}(1)-\mathrm{C}(75)$ & $1.521(4)$ & $\mathrm{C}(13)-\mathrm{B}(1)-\mathrm{C}(19)$ & $108.5(2)$ \\
\hline$C(61)-C(62)$ & $1.483(5)$ & $\mathrm{C}(7)-\mathrm{B}(1)-\mathrm{C}(1)$ & $106.9(2)$ \\
\hline $\mathrm{C}(61)-\mathrm{N}(2)$ & $1.532(4)$ & $\mathrm{C}(13)-\mathrm{B}(1)-\mathrm{C}(1)$ & 110.1(3) \\
\hline $\mathrm{C}(61)-\mathrm{H}(61 \mathrm{~A})$ & 0.9900 & $\mathrm{C}(19)-\mathrm{B}(1)-\mathrm{C}(1)$ & $109.9(3)$ \\
\hline $\mathrm{C}(61)-\mathrm{H}(61 \mathrm{~B})$ & 0.9900 & $\mathrm{C}(21)-\mathrm{P}(1)-\mathrm{C}(25)$ & $101.83(15)$ \\
\hline $\mathrm{C}(62)-\mathrm{H}(62 \mathrm{~A})$ & 0.9800 & $\mathrm{C}(21)-\mathrm{P}(1)-\mathrm{C}(28)$ & $104.15(14)$ \\
\hline $\mathrm{C}(62)-\mathrm{H}(62 \mathrm{~B})$ & 0.9800 & $\mathrm{C}(25)-\mathrm{P}(1)-\mathrm{C}(28)$ & $101.42(15)$ \\
\hline $\mathrm{C}(62)-\mathrm{H}(62 \mathrm{C})$ & 0.9800 & $\mathrm{C}(2)-\mathrm{C}(1)-\mathrm{C}(6)$ & $113.4(3)$ \\
\hline $\mathrm{C}(63)-\mathrm{C}(64)$ & $1.476(4)$ & $\mathrm{C}(2)-\mathrm{C}(1)-\mathrm{B}(1)$ & $123.4(3)$ \\
\hline $\mathrm{C}(63)-\mathrm{N}(2)$ & $1.547(4)$ & $\mathrm{C}(6)-\mathrm{C}(1)-\mathrm{B}(1)$ & $123.0(3)$ \\
\hline $\mathrm{C}(63)-\mathrm{H}(63 \mathrm{~A})$ & 0.9900 & $\mathrm{C}(1)-\mathrm{C}(2)-\mathrm{C}(3)$ & $124.3(3)$ \\
\hline $\mathrm{C}(63)-\mathrm{H}(63 \mathrm{~B})$ & 0.9900 & $\mathrm{C}(1)-\mathrm{C}(2)-\mathrm{H}(2)$ & 117.9 \\
\hline $\mathrm{C}(64)-\mathrm{H}(64 \mathrm{~A})$ & 0.9800 & $\mathrm{C}(3)-\mathrm{C}(2)-\mathrm{H}(2)$ & 117.9 \\
\hline $\mathrm{C}(64)-\mathrm{H}(64 \mathrm{~B})$ & 0.9800 & $C(4)-C(3)-C(2)$ & 119.8(3) \\
\hline $\mathrm{C}(64)-\mathrm{H}(64 \mathrm{C})$ & 0.9800 & $\mathrm{C}(4)-\mathrm{C}(3)-\mathrm{H}(3)$ & 120.1 \\
\hline $\mathrm{C}(65)-\mathrm{N}(2)$ & $1.489(4)$ & $\mathrm{C}(2)-\mathrm{C}(3)-\mathrm{H}(3)$ & 120.1 \\
\hline $\mathrm{C}(65)-\mathrm{C}(66)$ & $1.534(4)$ & $C(5)-C(4)-C(3)$ & $118.7(3)$ \\
\hline $\mathrm{C}(65)-\mathrm{H}(65 \mathrm{~A})$ & 0.9900 & $\mathrm{C}(5)-\mathrm{C}(4)-\mathrm{H}(4)$ & 120.7 \\
\hline $\mathrm{C}(65)-\mathrm{H}(65 \mathrm{~B})$ & 0.9900 & $\mathrm{C}(3)-\mathrm{C}(4)-\mathrm{H}(4)$ & 120.7 \\
\hline $\mathrm{C}(66)-\mathrm{H}(66 \mathrm{~A})$ & 0.9800 & $\mathrm{C}(4)-\mathrm{C}(5)-\mathrm{C}(6)$ & $120.2(3)$ \\
\hline $\mathrm{C}(66)-\mathrm{H}(66 \mathrm{~B})$ & 0.9800 & $\mathrm{C}(4)-\mathrm{C}(5)-\mathrm{H}(5)$ & 119.9 \\
\hline
\end{tabular}




\begin{tabular}{|c|c|}
\hline $\mathrm{C}(6)-\mathrm{C}(5)-\mathrm{H}(5)$ & 119.9 \\
\hline $\mathrm{C}(5)-\mathrm{C}(6)-\mathrm{C}(1)$ & $123.7(3)$ \\
\hline $\mathrm{C}(5)-\mathrm{C}(6)-\mathrm{H}(6)$ & 118.2 \\
\hline $\mathrm{C}(1)-\mathrm{C}(6)-\mathrm{H}(6)$ & 118.2 \\
\hline$C(12)-C(7)-C(8)$ & $114.6(3)$ \\
\hline $\mathrm{C}(12)-\mathrm{C}(7)-\mathrm{B}(1)$ & 125.1(3) \\
\hline $\mathrm{C}(8)-\mathrm{C}(7)-\mathrm{B}(1)$ & $120.3(3)$ \\
\hline $\mathrm{C}(9)-\mathrm{C}(8)-\mathrm{C}(7)$ & $123.0(3)$ \\
\hline $\mathrm{C}(9)-\mathrm{C}(8)-\mathrm{H}(8)$ & 118.5 \\
\hline $\mathrm{C}(7)-\mathrm{C}(8)-\mathrm{H}(8)$ & 118.5 \\
\hline $\mathrm{C}(8)-\mathrm{C}(9)-\mathrm{C}(10)$ & $120.5(3)$ \\
\hline $\mathrm{C}(8)-\mathrm{C}(9)-\mathrm{H}(9)$ & 119.8 \\
\hline $\mathrm{C}(10)-\mathrm{C}(9)-\mathrm{H}(9)$ & 119.8 \\
\hline $\mathrm{C}(11)-\mathrm{C}(10)-\mathrm{C}(9)$ & 118.3(3) \\
\hline $\mathrm{C}(11)-\mathrm{C}(10)-\mathrm{H}(10)$ & 120.8 \\
\hline $\mathrm{C}(9)-\mathrm{C}(10)-\mathrm{H}(10)$ & 120.8 \\
\hline $\mathrm{C}(10)-\mathrm{C}(11)-\mathrm{C}(12)$ & $119.8(3)$ \\
\hline $\mathrm{C}(10)-\mathrm{C}(11)-\mathrm{H}(11)$ & 120.1 \\
\hline $\mathrm{C}(12)-\mathrm{C}(11)-\mathrm{H}(11)$ & 120.1 \\
\hline $\mathrm{C}(11)-\mathrm{C}(12)-\mathrm{C}(7)$ & $123.8(3)$ \\
\hline $\mathrm{C}(11)-\mathrm{C}(12)-\mathrm{H}(12)$ & 118.1 \\
\hline $\mathrm{C}(7)-\mathrm{C}(12)-\mathrm{H}(12)$ & 118.1 \\
\hline $\mathrm{C}(14)-\mathrm{C}(13)-\mathrm{C}(18)$ & $115.3(3)$ \\
\hline $\mathrm{C}(14)-\mathrm{C}(13)-\mathrm{B}(1)$ & 121.3(3) \\
\hline $\mathrm{C}(18)-\mathrm{C}(13)-\mathrm{B}(1)$ & $123.0(3)$ \\
\hline$C(13)-C(14)-C(15)$ & $122.5(3)$ \\
\hline $\mathrm{C}(13)-\mathrm{C}(14)-\mathrm{H}(14)$ & 118.7 \\
\hline $\mathrm{C}(15)-\mathrm{C}(14)-\mathrm{H}(14)$ & 118.7 \\
\hline$C(16)-C(15)-C(14)$ & 119.1(3) \\
\hline $\mathrm{C}(16)-\mathrm{C}(15)-\mathrm{H}(15)$ & 120.5 \\
\hline $\mathrm{C}(14)-\mathrm{C}(15)-\mathrm{H}(15)$ & 120.5 \\
\hline $\mathrm{C}(15)-\mathrm{C}(16)-\mathrm{C}(17)$ & $120.9(3)$ \\
\hline $\mathrm{C}(15)-\mathrm{C}(16)-\mathrm{H}(16)$ & 119.5 \\
\hline $\mathrm{C}(17)-\mathrm{C}(16)-\mathrm{H}(16)$ & 119.5 \\
\hline$C(16)-C(17)-C(18)$ & $118.6(3)$ \\
\hline $\mathrm{C}(16)-\mathrm{C}(17)-\mathrm{H}(17)$ & 120.7 \\
\hline $\mathrm{C}(18)-\mathrm{C}(17)-\mathrm{H}(17)$ & 120.7 \\
\hline $\mathrm{C}(13)-\mathrm{C}(18)-\mathrm{C}(17)$ & $123.5(3)$ \\
\hline $\mathrm{C}(13)-\mathrm{C}(18)-\mathrm{H}(18)$ & 118.3 \\
\hline $\mathrm{C}(17)-\mathrm{C}(18)-\mathrm{H}(18)$ & 118.3 \\
\hline$C(24)-C(19)-C(20)$ & $114.4(3)$ \\
\hline $\mathrm{C}(24)-\mathrm{C}(19)-\mathrm{B}(1)$ & $121.9(3)$ \\
\hline $\mathrm{C}(20)-\mathrm{C}(19)-\mathrm{B}(1)$ & $123.7(3)$ \\
\hline$C(19)-C(20)-C(21)$ & $125.7(3)$ \\
\hline $\mathrm{C}(19)-\mathrm{C}(20)-\mathrm{H}(20)$ & 117.2 \\
\hline $\mathrm{C}(21)-\mathrm{C}(20)-\mathrm{H}(20)$ & 117.2 \\
\hline $\mathrm{C}(22)-\mathrm{C}(21)-\mathrm{C}(20)$ & $116.2(3)$ \\
\hline $\mathrm{C}(22)-\mathrm{C}(21)-\mathrm{P}(1)$ & $125.9(2)$ \\
\hline $\mathrm{C}(20)-\mathrm{C}(21)-\mathrm{P}(1)$ & $117.9(2)$ \\
\hline $\mathrm{C}(23)-\mathrm{C}(22)-\mathrm{C}(21)$ & $120.6(3)$ \\
\hline $\mathrm{C}(23)-\mathrm{C}(22)-\mathrm{H}(22)$ & 119.7 \\
\hline $\mathrm{C}(21)-\mathrm{C}(22)-\mathrm{H}(22)$ & 119.7 \\
\hline $\mathrm{C}(22)-\mathrm{C}(23)-\mathrm{C}(24)$ & $120.8(3)$ \\
\hline $\mathrm{C}(22)-\mathrm{C}(23)-\mathrm{H}(23)$ & 119.6 \\
\hline $\mathrm{C}(24)-\mathrm{C}(23)-\mathrm{H}(23)$ & 119.6 \\
\hline$C(19)-C(24)-C(23)$ & $122.2(3)$ \\
\hline
\end{tabular}

\begin{tabular}{|c|c|}
\hline $\mathrm{C}(19)-\mathrm{C}(24)-\mathrm{H}(24)$ & 118.9 \\
\hline $\mathrm{C}(23)-\mathrm{C}(24)-\mathrm{H}(24)$ & 118.9 \\
\hline $\mathrm{C}(27)-\mathrm{C}(25)-\mathrm{C}(26)$ & $110.7(3)$ \\
\hline $\mathrm{C}(27)-\mathrm{C}(25)-\mathrm{P}(1)$ & $116.6(2)$ \\
\hline $\mathrm{C}(26)-\mathrm{C}(25)-\mathrm{P}(1)$ & $109.0(2)$ \\
\hline $\mathrm{C}(27)-\mathrm{C}(25)-\mathrm{H}(25)$ & 106.7 \\
\hline $\mathrm{C}(26)-\mathrm{C}(25)-\mathrm{H}(25)$ & 106.7 \\
\hline $\mathrm{P}(1)-\mathrm{C}(25)-\mathrm{H}(25)$ & 106.7 \\
\hline $\mathrm{C}(25)-\mathrm{C}(26)-\mathrm{H}(26 \mathrm{~A})$ & 109.5 \\
\hline $\mathrm{C}(25)-\mathrm{C}(26)-\mathrm{H}(26 \mathrm{~B})$ & 109.5 \\
\hline $\mathrm{H}(26 \mathrm{~A})-\mathrm{C}(26)-\mathrm{H}(26 \mathrm{~B})$ & 109.5 \\
\hline $\mathrm{C}(25)-\mathrm{C}(26)-\mathrm{H}(26 \mathrm{C})$ & 109.5 \\
\hline $\mathrm{H}(26 \mathrm{~A})-\mathrm{C}(26)-\mathrm{H}(26 \mathrm{C})$ & 109.5 \\
\hline $\mathrm{H}(26 \mathrm{~B})-\mathrm{C}(26)-\mathrm{H}(26 \mathrm{C})$ & 109.5 \\
\hline $\mathrm{C}(25)-\mathrm{C}(27)-\mathrm{H}(27 \mathrm{~A})$ & 109.5 \\
\hline $\mathrm{C}(25)-\mathrm{C}(27)-\mathrm{H}(27 \mathrm{~B})$ & 109.5 \\
\hline $\mathrm{H}(27 \mathrm{~A})-\mathrm{C}(27)-\mathrm{H}(27 \mathrm{~B})$ & 109.5 \\
\hline $\mathrm{C}(25)-\mathrm{C}(27)-\mathrm{H}(27 \mathrm{C})$ & 109.5 \\
\hline $\mathrm{H}(27 \mathrm{~A})-\mathrm{C}(27)-\mathrm{H}(27 \mathrm{C})$ & 109.5 \\
\hline $\mathrm{H}(27 \mathrm{~B})-\mathrm{C}(27)-\mathrm{H}(27 \mathrm{C})$ & 109.5 \\
\hline C(29)-C(28)-C(30) & $109.0(3)$ \\
\hline $\mathrm{C}(29)-\mathrm{C}(28)-\mathrm{P}(1)$ & $110.0(2)$ \\
\hline $\mathrm{C}(30)-\mathrm{C}(28)-\mathrm{P}(1)$ & $108.7(2)$ \\
\hline $\mathrm{C}(29)-\mathrm{C}(28)-\mathrm{H}(28)$ & 109.7 \\
\hline $\mathrm{C}(30)-\mathrm{C}(28)-\mathrm{H}(28)$ & 109.7 \\
\hline $\mathrm{P}(1)-\mathrm{C}(28)-\mathrm{H}(28)$ & 109.7 \\
\hline $\mathrm{C}(28)-\mathrm{C}(29)-\mathrm{H}(29 \mathrm{~A})$ & 109.5 \\
\hline $\mathrm{C}(28)-\mathrm{C}(29)-\mathrm{H}(29 \mathrm{~B})$ & 109.5 \\
\hline $\mathrm{H}(29 \mathrm{~A})-\mathrm{C}(29)-\mathrm{H}(29 \mathrm{~B})$ & 109.5 \\
\hline $\mathrm{C}(28)-\mathrm{C}(29)-\mathrm{H}(29 \mathrm{C})$ & 109.5 \\
\hline $\mathrm{H}(29 \mathrm{~A})-\mathrm{C}(29)-\mathrm{H}(29 \mathrm{C})$ & 109.5 \\
\hline $\mathrm{H}(29 \mathrm{~B})-\mathrm{C}(29)-\mathrm{H}(29 \mathrm{C})$ & 109.5 \\
\hline $\mathrm{C}(28)-\mathrm{C}(30)-\mathrm{H}(30 \mathrm{~A})$ & 109.5 \\
\hline $\mathrm{C}(28)-\mathrm{C}(30)-\mathrm{H}(30 \mathrm{~B})$ & 109.5 \\
\hline $\mathrm{H}(30 \mathrm{~A})-\mathrm{C}(30)-\mathrm{H}(30 \mathrm{~B})$ & 109.5 \\
\hline $\mathrm{C}(28)-\mathrm{C}(30)-\mathrm{H}(30 \mathrm{C})$ & 109.5 \\
\hline $\mathrm{H}(30 \mathrm{~A})-\mathrm{C}(30)-\mathrm{H}(30 \mathrm{C})$ & 109.5 \\
\hline $\mathrm{H}(30 \mathrm{~B})-\mathrm{C}(30)-\mathrm{H}(30 \mathrm{C})$ & 109.5 \\
\hline $\mathrm{C}(37)-\mathrm{B}(2)-\mathrm{C}(49)$ & $108.5(2)$ \\
\hline $\mathrm{C}(37)-\mathrm{B}(2)-\mathrm{C}(43)$ & 110.1(3) \\
\hline $\mathrm{C}(49)-\mathrm{B}(2)-\mathrm{C}(43)$ & $111.7(3)$ \\
\hline $\mathrm{C}(37)-\mathrm{B}(2)-\mathrm{C}(31)$ & $108.2(3)$ \\
\hline $\mathrm{C}(49)-\mathrm{B}(2)-\mathrm{C}(31)$ & $111.5(3)$ \\
\hline $\mathrm{C}(43)-\mathrm{B}(2)-\mathrm{C}(31)$ & $106.8(2)$ \\
\hline $\mathrm{C}(58)-\mathrm{P}(2)-\mathrm{C}(51)$ & $103.74(14)$ \\
\hline $\mathrm{C}(58)-\mathrm{P}(2)-\mathrm{C}(55)$ & $101.74(15)$ \\
\hline $\mathrm{C}(51)-\mathrm{P}(2)-\mathrm{C}(55)$ & $101.93(14)$ \\
\hline $\mathrm{C}(32)-\mathrm{C}(31)-\mathrm{C}(36)$ & $114.0(3)$ \\
\hline $\mathrm{C}(32)-\mathrm{C}(31)-\mathrm{B}(2)$ & $124.8(3)$ \\
\hline $\mathrm{C}(36)-\mathrm{C}(31)-\mathrm{B}(2)$ & $121.2(3)$ \\
\hline $\mathrm{C}(33)-\mathrm{C}(32)-\mathrm{C}(31)$ & $123.7(3)$ \\
\hline $\mathrm{C}(33)-\mathrm{C}(32)-\mathrm{H}(32)$ & 118.2 \\
\hline $\mathrm{C}(31)-\mathrm{C}(32)-\mathrm{H}(32)$ & 118.2 \\
\hline $\mathrm{C}(34)-\mathrm{C}(33)-\mathrm{C}(32)$ & $120.3(3)$ \\
\hline $\mathrm{C}(34)-\mathrm{C}(33)-\mathrm{H}(33)$ & 119.9 \\
\hline $\mathrm{C}(32)-\mathrm{C}(33)-\mathrm{H}(33)$ & 119.9 \\
\hline
\end{tabular}




\begin{tabular}{|c|c|}
\hline$C(33)-C(34)-C(35)$ & $117.9(3)$ \\
\hline $\mathrm{C}(33)-\mathrm{C}(34)-\mathrm{H}(34)$ & 121.0 \\
\hline $\mathrm{C}(35)-\mathrm{C}(34)-\mathrm{H}(34)$ & 121.0 \\
\hline $\mathrm{C}(36)-\mathrm{C}(35)-\mathrm{C}(34)$ & $120.6(3)$ \\
\hline $\mathrm{C}(36)-\mathrm{C}(35)-\mathrm{H}(35)$ & 119.7 \\
\hline $\mathrm{C}(34)-\mathrm{C}(35)-\mathrm{H}(35)$ & 119.7 \\
\hline $\mathrm{C}(35)-\mathrm{C}(36)-\mathrm{C}(31)$ & $123.5(3)$ \\
\hline $\mathrm{C}(35)-\mathrm{C}(36)-\mathrm{H}(36)$ & 118.3 \\
\hline $\mathrm{C}(31)-\mathrm{C}(36)-\mathrm{H}(36)$ & 118.3 \\
\hline $\mathrm{C}(42)-\mathrm{C}(37)-\mathrm{C}(38)$ & $114.0(3)$ \\
\hline $\mathrm{C}(42)-\mathrm{C}(37)-\mathrm{B}(2)$ & $123.3(3)$ \\
\hline $\mathrm{C}(38)-\mathrm{C}(37)-\mathrm{B}(2)$ & $122.2(3)$ \\
\hline $\mathrm{C}(39)-\mathrm{C}(38)-\mathrm{C}(37)$ & $123.6(3)$ \\
\hline $\mathrm{C}(39)-\mathrm{C}(38)-\mathrm{H}(38)$ & 118.2 \\
\hline $\mathrm{C}(37)-\mathrm{C}(38)-\mathrm{H}(38)$ & 118.2 \\
\hline $\mathrm{C}(40)-\mathrm{C}(39)-\mathrm{C}(38)$ & $119.9(3)$ \\
\hline $\mathrm{C}(40)-\mathrm{C}(39)-\mathrm{H}(39)$ & 120.1 \\
\hline $\mathrm{C}(38)-\mathrm{C}(39)-\mathrm{H}(39)$ & 120.1 \\
\hline $\mathrm{C}(39)-\mathrm{C}(40)-\mathrm{C}(41)$ & $118.8(3)$ \\
\hline $\mathrm{C}(39)-\mathrm{C}(40)-\mathrm{H}(40)$ & 120.6 \\
\hline $\mathrm{C}(41)-\mathrm{C}(40)-\mathrm{H}(40)$ & 120.6 \\
\hline$C(40)-C(41)-C(42)$ & $120.9(3)$ \\
\hline $\mathrm{C}(40)-\mathrm{C}(41)-\mathrm{H}(41)$ & 119.6 \\
\hline $\mathrm{C}(42)-\mathrm{C}(41)-\mathrm{H}(41)$ & 119.6 \\
\hline $\mathrm{C}(41)-\mathrm{C}(42)-\mathrm{C}(37)$ & $122.8(3)$ \\
\hline $\mathrm{C}(41)-\mathrm{C}(42)-\mathrm{H}(42)$ & 118.6 \\
\hline $\mathrm{C}(37)-\mathrm{C}(42)-\mathrm{H}(42)$ & 118.6 \\
\hline $\mathrm{C}(48)-\mathrm{C}(43)-\mathrm{C}(44)$ & $114.5(3)$ \\
\hline $\mathrm{C}(48)-\mathrm{C}(43)-\mathrm{B}(2)$ & $124.9(3)$ \\
\hline $\mathrm{C}(44)-\mathrm{C}(43)-\mathrm{B}(2)$ & $120.6(3)$ \\
\hline$C(45)-C(44)-C(43)$ & $122.5(3)$ \\
\hline $\mathrm{C}(45)-\mathrm{C}(44)-\mathrm{H}(44)$ & 118.8 \\
\hline $\mathrm{C}(43)-\mathrm{C}(44)-\mathrm{H}(44)$ & 118.8 \\
\hline$C(46)-C(45)-C(44)$ & 121.2(3) \\
\hline $\mathrm{C}(46)-\mathrm{C}(45)-\mathrm{H}(45)$ & 119.4 \\
\hline $\mathrm{C}(44)-\mathrm{C}(45)-\mathrm{H}(45)$ & 119.4 \\
\hline$C(47)-C(46)-C(45)$ & $118.7(3)$ \\
\hline $\mathrm{C}(47)-\mathrm{C}(46)-\mathrm{H}(46)$ & 120.7 \\
\hline $\mathrm{C}(45)-\mathrm{C}(46)-\mathrm{H}(46)$ & 120.7 \\
\hline$C(46)-C(47)-C(48)$ & 120.3(3) \\
\hline $\mathrm{C}(46)-\mathrm{C}(47)-\mathrm{H}(47)$ & 119.9 \\
\hline $\mathrm{C}(48)-\mathrm{C}(47)-\mathrm{H}(47)$ & 119.9 \\
\hline $\mathrm{C}(47)-\mathrm{C}(48)-\mathrm{C}(43)$ & $122.9(3)$ \\
\hline $\mathrm{C}(47)-\mathrm{C}(48)-\mathrm{H}(48)$ & 118.6 \\
\hline $\mathrm{C}(43)-\mathrm{C}(48)-\mathrm{H}(48)$ & 118.6 \\
\hline$C(50)-C(49)-C(54)$ & $113.3(3)$ \\
\hline $\mathrm{C}(50)-\mathrm{C}(49)-\mathrm{B}(2)$ & $123.5(3)$ \\
\hline C(54)-C(49)-B(2) & $123.2(3)$ \\
\hline$C(51)-C(50)-C(49)$ & $125.6(3)$ \\
\hline $\mathrm{C}(51)-\mathrm{C}(50)-\mathrm{H}(50)$ & 117.2 \\
\hline $\mathrm{C}(49)-\mathrm{C}(50)-\mathrm{H}(50)$ & 117.2 \\
\hline$C(50)-C(51)-C(52)$ & $117.9(3)$ \\
\hline $\mathrm{C}(50)-\mathrm{C}(51)-\mathrm{P}(2)$ & 117.1(2) \\
\hline $\mathrm{C}(52)-\mathrm{C}(51)-\mathrm{P}(2)$ & $125.1(2)$ \\
\hline $\mathrm{C}(53)-\mathrm{C}(52)-\mathrm{C}(51)$ & 119.3(3) \\
\hline $\mathrm{C}(53)-\mathrm{C}(52)-\mathrm{H}(52)$ & 120.3 \\
\hline
\end{tabular}

\begin{tabular}{|c|c|}
\hline $\mathrm{C}(51)-\mathrm{C}(52)-\mathrm{H}(52)$ & 120.3 \\
\hline $\mathrm{C}(54)-\mathrm{C}(53)-\mathrm{C}(52)$ & $120.5(3)$ \\
\hline $\mathrm{C}(54)-\mathrm{C}(53)-\mathrm{H}(53)$ & 119.7 \\
\hline $\mathrm{C}(52)-\mathrm{C}(53)-\mathrm{H}(53)$ & 119.7 \\
\hline $\mathrm{C}(53)-\mathrm{C}(54)-\mathrm{C}(49)$ & $123.4(3)$ \\
\hline $\mathrm{C}(53)-\mathrm{C}(54)-\mathrm{H}(54)$ & 118.3 \\
\hline $\mathrm{C}(49)-\mathrm{C}(54)-\mathrm{H}(54)$ & 118.3 \\
\hline$C(57)-C(55)-C(56)$ & $109.8(3)$ \\
\hline $\mathrm{C}(57)-\mathrm{C}(55)-\mathrm{P}(2)$ & $116.9(2)$ \\
\hline $\mathrm{C}(56)-\mathrm{C}(55)-\mathrm{P}(2)$ & $109.7(2)$ \\
\hline $\mathrm{C}(57)-\mathrm{C}(55)-\mathrm{H}(55)$ & 106.6 \\
\hline $\mathrm{C}(56)-\mathrm{C}(55)-\mathrm{H}(55)$ & 106.6 \\
\hline $\mathrm{P}(2)-\mathrm{C}(55)-\mathrm{H}(55)$ & 106.6 \\
\hline $\mathrm{C}(55)-\mathrm{C}(56)-\mathrm{H}(56 \mathrm{~A})$ & 109.5 \\
\hline $\mathrm{C}(55)-\mathrm{C}(56)-\mathrm{H}(56 \mathrm{~B})$ & 109.5 \\
\hline $\mathrm{H}(56 \mathrm{~A})-\mathrm{C}(56)-\mathrm{H}(56 \mathrm{~B})$ & 109.5 \\
\hline $\mathrm{C}(55)-\mathrm{C}(56)-\mathrm{H}(56 \mathrm{C})$ & 109.5 \\
\hline $\mathrm{H}(56 \mathrm{~A})-\mathrm{C}(56)-\mathrm{H}(56 \mathrm{C})$ & 109.5 \\
\hline $\mathrm{H}(56 \mathrm{~B})-\mathrm{C}(56)-\mathrm{H}(56 \mathrm{C})$ & 109.5 \\
\hline $\mathrm{C}(55)-\mathrm{C}(57)-\mathrm{H}(57 \mathrm{~A})$ & 109.5 \\
\hline $\mathrm{C}(55)-\mathrm{C}(57)-\mathrm{H}(57 \mathrm{~B})$ & 109.5 \\
\hline $\mathrm{H}(57 \mathrm{~A})-\mathrm{C}(57)-\mathrm{H}(57 \mathrm{~B})$ & 109.5 \\
\hline $\mathrm{C}(55)-\mathrm{C}(57)-\mathrm{H}(57 \mathrm{C})$ & 109.5 \\
\hline $\mathrm{H}(57 \mathrm{~A})-\mathrm{C}(57)-\mathrm{H}(57 \mathrm{C})$ & 109.5 \\
\hline $\mathrm{H}(57 \mathrm{~B})-\mathrm{C}(57)-\mathrm{H}(57 \mathrm{C})$ & 109.5 \\
\hline $\mathrm{C}(60)-\mathrm{C}(58)-\mathrm{C}(59)$ & 109.6(3) \\
\hline $\mathrm{C}(60)-\mathrm{C}(58)-\mathrm{P}(2)$ & $111.8(2)$ \\
\hline $\mathrm{C}(59)-\mathrm{C}(58)-\mathrm{P}(2)$ & $109.4(2)$ \\
\hline $\mathrm{C}(60)-\mathrm{C}(58)-\mathrm{H}(58)$ & 108.7 \\
\hline $\mathrm{C}(59)-\mathrm{C}(58)-\mathrm{H}(58)$ & 108.7 \\
\hline $\mathrm{P}(2)-\mathrm{C}(58)-\mathrm{H}(58)$ & 108.7 \\
\hline $\mathrm{C}(58)-\mathrm{C}(59)-\mathrm{H}(59 \mathrm{~A})$ & 109.5 \\
\hline $\mathrm{C}(58)-\mathrm{C}(59)-\mathrm{H}(59 \mathrm{~B})$ & 109.5 \\
\hline $\mathrm{H}(59 \mathrm{~A})-\mathrm{C}(59)-\mathrm{H}(59 \mathrm{~B})$ & 109.5 \\
\hline $\mathrm{C}(58)-\mathrm{C}(59)-\mathrm{H}(59 \mathrm{C})$ & 109.5 \\
\hline $\mathrm{H}(59 \mathrm{~A})-\mathrm{C}(59)-\mathrm{H}(59 \mathrm{C})$ & 109.5 \\
\hline $\mathrm{H}(59 \mathrm{~B})-\mathrm{C}(59)-\mathrm{H}(59 \mathrm{C})$ & 109.5 \\
\hline $\mathrm{C}(58)-\mathrm{C}(60)-\mathrm{H}(60 \mathrm{~A})$ & 109.5 \\
\hline $\mathrm{C}(58)-\mathrm{C}(60)-\mathrm{H}(60 \mathrm{~B})$ & 109.5 \\
\hline $\mathrm{H}(60 \mathrm{~A})-\mathrm{C}(60)-\mathrm{H}(60 \mathrm{~B})$ & 109.5 \\
\hline $\mathrm{C}(58)-\mathrm{C}(60)-\mathrm{H}(60 \mathrm{C})$ & 109.5 \\
\hline $\mathrm{H}(60 \mathrm{~A})-\mathrm{C}(60)-\mathrm{H}(60 \mathrm{C})$ & 109.5 \\
\hline $\mathrm{H}(60 \mathrm{~B})-\mathrm{C}(60)-\mathrm{H}(60 \mathrm{C})$ & 109.5 \\
\hline $\mathrm{C}(73)-\mathrm{N}(1)-\mathrm{C}(71)$ & $106.3(3)$ \\
\hline $\mathrm{C}(73)-\mathrm{N}(1)-\mathrm{C}(69)$ & $112.7(2)$ \\
\hline $\mathrm{C}(71)-\mathrm{N}(1)-\mathrm{C}(69)$ & $110.1(3)$ \\
\hline $\mathrm{C}(73)-\mathrm{N}(1)-\mathrm{C}(75)$ & $111.8(3)$ \\
\hline $\mathrm{C}(71)-\mathrm{N}(1)-\mathrm{C}(75)$ & $110.5(3)$ \\
\hline $\mathrm{C}(69)-\mathrm{N}(1)-\mathrm{C}(75)$ & $105.4(2)$ \\
\hline $\mathrm{C}(62)-\mathrm{C}(61)-\mathrm{N}(2)$ & $118.4(3)$ \\
\hline $\mathrm{C}(62)-\mathrm{C}(61)-\mathrm{H}(61 \mathrm{~A})$ & 107.7 \\
\hline $\mathrm{N}(2)-\mathrm{C}(61)-\mathrm{H}(61 \mathrm{~A})$ & 107.7 \\
\hline $\mathrm{C}(62)-\mathrm{C}(61)-\mathrm{H}(61 \mathrm{~B})$ & 107.7 \\
\hline N(2)-C(61)-H(61B) & 107.7 \\
\hline $\mathrm{H}(61 \mathrm{~A})-\mathrm{C}(61)-\mathrm{H}(61 \mathrm{~B})$ & 107.1 \\
\hline $\mathrm{C}(61)-\mathrm{C}(62)-\mathrm{H}(62 \mathrm{~A})$ & 109.5 \\
\hline
\end{tabular}




\begin{tabular}{|c|c|c|c|}
\hline $\mathrm{C}(61)-\mathrm{C}(62)-\mathrm{H}(62 \mathrm{~B})$ & 109.5 & $\mathrm{C}(70)-\mathrm{C}(69)-\mathrm{H}(69 \mathrm{~A})$ & 108.5 \\
\hline $\mathrm{H}(62 \mathrm{~A})-\mathrm{C}(62)-\mathrm{H}(62 \mathrm{~B})$ & 109.5 & $\mathrm{~N}(1)-\mathrm{C}(69)-\mathrm{H}(69 \mathrm{~A})$ & 108.5 \\
\hline $\mathrm{C}(61)-\mathrm{C}(62)-\mathrm{H}(62 \mathrm{C})$ & 109.5 & $\mathrm{C}(70)-\mathrm{C}(69)-\mathrm{H}(69 \mathrm{~B})$ & 108.5 \\
\hline $\mathrm{H}(62 \mathrm{~A})-\mathrm{C}(62)-\mathrm{H}(62 \mathrm{C})$ & 109.5 & $\mathrm{~N}(1)-\mathrm{C}(69)-\mathrm{H}(69 \mathrm{~B})$ & 108.5 \\
\hline $\mathrm{H}(62 \mathrm{~B})-\mathrm{C}(62)-\mathrm{H}(62 \mathrm{C})$ & 109.5 & $\mathrm{H}(69 \mathrm{~A})-\mathrm{C}(69)-\mathrm{H}(69 \mathrm{~B})$ & 107.5 \\
\hline $\mathrm{C}(64)-\mathrm{C}(63)-\mathrm{N}(2)$ & $115.1(3)$ & $\mathrm{C}(69)-\mathrm{C}(70)-\mathrm{H}(70 \mathrm{~A})$ & 109.5 \\
\hline $\mathrm{C}(64)-\mathrm{C}(63)-\mathrm{H}(63 \mathrm{~A})$ & 108.5 & $\mathrm{C}(69)-\mathrm{C}(70)-\mathrm{H}(70 \mathrm{~B})$ & 109.5 \\
\hline $\mathrm{N}(2)-\mathrm{C}(63)-\mathrm{H}(63 \mathrm{~A})$ & 108.5 & $\mathrm{H}(70 \mathrm{~A})-\mathrm{C}(70)-\mathrm{H}(70 \mathrm{~B})$ & 109.5 \\
\hline $\mathrm{C}(64)-\mathrm{C}(63)-\mathrm{H}(63 \mathrm{~B})$ & 108.5 & $\mathrm{C}(69)-\mathrm{C}(70)-\mathrm{H}(70 \mathrm{C})$ & 109.5 \\
\hline $\mathrm{N}(2)-\mathrm{C}(63)-\mathrm{H}(63 \mathrm{~B})$ & 108.5 & $\mathrm{H}(70 \mathrm{~A})-\mathrm{C}(70)-\mathrm{H}(70 \mathrm{C})$ & 109.5 \\
\hline $\mathrm{H}(63 \mathrm{~A})-\mathrm{C}(63)-\mathrm{H}(63 \mathrm{~B})$ & 107.5 & $\mathrm{H}(70 \mathrm{~B})-\mathrm{C}(70)-\mathrm{H}(70 \mathrm{C})$ & 109.5 \\
\hline $\mathrm{C}(63)-\mathrm{C}(64)-\mathrm{H}(64 \mathrm{~A})$ & 109.5 & $\mathrm{~N}(1)-\mathrm{C}(71)-\mathrm{C}(72)$ & $117.0(3)$ \\
\hline $\mathrm{C}(63)-\mathrm{C}(64)-\mathrm{H}(64 \mathrm{~B})$ & 109.5 & $\mathrm{~N}(1)-\mathrm{C}(71)-\mathrm{H}(71 \mathrm{~A})$ & 108.1 \\
\hline $\mathrm{H}(64 \mathrm{~A})-\mathrm{C}(64)-\mathrm{H}(64 \mathrm{~B})$ & 109.5 & $\mathrm{C}(72)-\mathrm{C}(71)-\mathrm{H}(71 \mathrm{~A})$ & 108.1 \\
\hline $\mathrm{C}(63)-\mathrm{C}(64)-\mathrm{H}(64 \mathrm{C})$ & 109.5 & $\mathrm{~N}(1)-\mathrm{C}(71)-\mathrm{H}(71 \mathrm{~B})$ & 108.1 \\
\hline $\mathrm{H}(64 \mathrm{~A})-\mathrm{C}(64)-\mathrm{H}(64 \mathrm{C})$ & 109.5 & $\mathrm{C}(72)-\mathrm{C}(71)-\mathrm{H}(71 \mathrm{~B})$ & 108.1 \\
\hline $\mathrm{H}(64 \mathrm{~B})-\mathrm{C}(64)-\mathrm{H}(64 \mathrm{C})$ & 109.5 & $\mathrm{H}(71 \mathrm{~A})-\mathrm{C}(71)-\mathrm{H}(71 \mathrm{~B})$ & 107.3 \\
\hline$N(2)-C(65)-C(66)$ & $114.6(3)$ & $\mathrm{C}(71)-\mathrm{C}(72)-\mathrm{H}(72 \mathrm{~A})$ & 109.5 \\
\hline $\mathrm{N}(2)-\mathrm{C}(65)-\mathrm{H}(65 \mathrm{~A})$ & 108.6 & $\mathrm{C}(71)-\mathrm{C}(72)-\mathrm{H}(72 \mathrm{~B})$ & 109.5 \\
\hline $\mathrm{C}(66)-\mathrm{C}(65)-\mathrm{H}(65 \mathrm{~A})$ & 108.6 & $\mathrm{H}(72 \mathrm{~A})-\mathrm{C}(72)-\mathrm{H}(72 \mathrm{~B})$ & 109.5 \\
\hline $\mathrm{N}(2)-\mathrm{C}(65)-\mathrm{H}(65 \mathrm{~B})$ & 108.6 & $\mathrm{C}(71)-\mathrm{C}(72)-\mathrm{H}(72 \mathrm{C})$ & 109.5 \\
\hline $\mathrm{C}(66)-\mathrm{C}(65)-\mathrm{H}(65 \mathrm{~B})$ & 108.6 & $\mathrm{H}(72 \mathrm{~A})-\mathrm{C}(72)-\mathrm{H}(72 \mathrm{C})$ & 109.5 \\
\hline $\mathrm{H}(65 \mathrm{~A})-\mathrm{C}(65)-\mathrm{H}(65 \mathrm{~B})$ & 107.6 & $\mathrm{H}(72 \mathrm{~B})-\mathrm{C}(72)-\mathrm{H}(72 \mathrm{C})$ & 109.5 \\
\hline $\mathrm{C}(65)-\mathrm{C}(66)-\mathrm{H}(66 \mathrm{~A})$ & 109.5 & $\mathrm{C}(74)-\mathrm{C}(73)-\mathrm{N}(1)$ & $115.7(3)$ \\
\hline $\mathrm{C}(65)-\mathrm{C}(66)-\mathrm{H}(66 \mathrm{~B})$ & 109.5 & $\mathrm{C}(74)-\mathrm{C}(73)-\mathrm{H}(73 \mathrm{~A})$ & 108.4 \\
\hline $\mathrm{H}(66 \mathrm{~A})-\mathrm{C}(66)-\mathrm{H}(66 \mathrm{~B})$ & 109.5 & $\mathrm{~N}(1)-\mathrm{C}(73)-\mathrm{H}(73 \mathrm{~A})$ & 108.4 \\
\hline $\mathrm{C}(65)-\mathrm{C}(66)-\mathrm{H}(66 \mathrm{C})$ & 109.5 & $\mathrm{C}(74)-\mathrm{C}(73)-\mathrm{H}(73 \mathrm{~B})$ & 108.4 \\
\hline $\mathrm{H}(66 \mathrm{~A})-\mathrm{C}(66)-\mathrm{H}(66 \mathrm{C})$ & 109.5 & $\mathrm{~N}(1)-\mathrm{C}(73)-\mathrm{H}(73 \mathrm{~B})$ & 108.4 \\
\hline $\mathrm{H}(66 \mathrm{~B})-\mathrm{C}(66)-\mathrm{H}(66 \mathrm{C})$ & 109.5 & $\mathrm{H}(73 \mathrm{~A})-\mathrm{C}(73)-\mathrm{H}(73 \mathrm{~B})$ & 107.4 \\
\hline$N(2)-C(67)-C(68)$ & $114.9(3)$ & $\mathrm{C}(73)-\mathrm{C}(74)-\mathrm{H}(74 \mathrm{~A})$ & 109.5 \\
\hline $\mathrm{N}(2)-\mathrm{C}(67)-\mathrm{H}(67 \mathrm{~A})$ & 108.6 & $\mathrm{C}(73)-\mathrm{C}(74)-\mathrm{H}(74 \mathrm{~B})$ & 109.5 \\
\hline $\mathrm{C}(68)-\mathrm{C}(67)-\mathrm{H}(67 \mathrm{~A})$ & 108.6 & $\mathrm{H}(74 \mathrm{~A})-\mathrm{C}(74)-\mathrm{H}(74 \mathrm{~B})$ & 109.5 \\
\hline $\mathrm{N}(2)-\mathrm{C}(67)-\mathrm{H}(67 \mathrm{~B})$ & 108.6 & $\mathrm{C}(73)-\mathrm{C}(74)-\mathrm{H}(74 \mathrm{C})$ & 109.5 \\
\hline $\mathrm{C}(68)-\mathrm{C}(67)-\mathrm{H}(67 \mathrm{~B})$ & 108.6 & $\mathrm{H}(74 \mathrm{~A})-\mathrm{C}(74)-\mathrm{H}(74 \mathrm{C})$ & 109.5 \\
\hline $\mathrm{H}(67 \mathrm{~A})-\mathrm{C}(67)-\mathrm{H}(67 \mathrm{~B})$ & 107.5 & $\mathrm{H}(74 \mathrm{~B})-\mathrm{C}(74)-\mathrm{H}(74 \mathrm{C})$ & 109.5 \\
\hline $\mathrm{C}(67)-\mathrm{C}(68)-\mathrm{H}(68 \mathrm{~A})$ & 109.5 & $\mathrm{C}(76)-\mathrm{C}(75)-\mathrm{N}(1)$ & $117.4(3)$ \\
\hline $\mathrm{C}(67)-\mathrm{C}(68)-\mathrm{H}(68 \mathrm{~B})$ & 109.5 & $\mathrm{C}(76)-\mathrm{C}(75)-\mathrm{H}(75 \mathrm{~A})$ & 108.0 \\
\hline $\mathrm{H}(68 \mathrm{~A})-\mathrm{C}(68)-\mathrm{H}(68 \mathrm{~B})$ & 109.5 & $\mathrm{~N}(1)-\mathrm{C}(75)-\mathrm{H}(75 \mathrm{~A})$ & 108.0 \\
\hline $\mathrm{C}(67)-\mathrm{C}(68)-\mathrm{H}(68 \mathrm{C})$ & 109.5 & $\mathrm{C}(76)-\mathrm{C}(75)-\mathrm{H}(75 \mathrm{~B})$ & 108.0 \\
\hline $\mathrm{H}(68 \mathrm{~A})-\mathrm{C}(68)-\mathrm{H}(68 \mathrm{C})$ & 109.5 & $\mathrm{~N}(1)-\mathrm{C}(75)-\mathrm{H}(75 \mathrm{~B})$ & 108.0 \\
\hline $\mathrm{H}(68 \mathrm{~B})-\mathrm{C}(68)-\mathrm{H}(68 \mathrm{C})$ & 109.5 & $\mathrm{H}(75 \mathrm{~A})-\mathrm{C}(75)-\mathrm{H}(75 \mathrm{~B})$ & 107.2 \\
\hline $\mathrm{C}(67)-\mathrm{N}(2)-\mathrm{C}(65)$ & $113.6(3)$ & $\mathrm{C}(75)-\mathrm{C}(76)-\mathrm{H}(76 \mathrm{~A})$ & 109.5 \\
\hline $\mathrm{C}(67)-\mathrm{N}(2)-\mathrm{C}(61)$ & $109.2(3)$ & $\mathrm{C}(75)-\mathrm{C}(76)-\mathrm{H}(76 \mathrm{~B})$ & 109.5 \\
\hline $\mathrm{C}(65)-\mathrm{N}(2)-\mathrm{C}(61)$ & $107.9(3)$ & $\mathrm{H}(76 \mathrm{~A})-\mathrm{C}(76)-\mathrm{H}(76 \mathrm{~B})$ & 109.5 \\
\hline $\mathrm{C}(67)-\mathrm{N}(2)-\mathrm{C}(63)$ & $108.7(2)$ & $\mathrm{C}(75)-\mathrm{C}(76)-\mathrm{H}(76 \mathrm{C})$ & 109.5 \\
\hline $\mathrm{C}(65)-\mathrm{N}(2)-\mathrm{C}(63)$ & $109.0(3)$ & $\mathrm{H}(76 \mathrm{~A})-\mathrm{C}(76)-\mathrm{H}(76 \mathrm{C})$ & 109.5 \\
\hline $\mathrm{C}(61)-\mathrm{N}(2)-\mathrm{C}(63)$ & $108.4(3)$ & $\mathrm{H}(76 \mathrm{~B})-\mathrm{C}(76)-\mathrm{H}(76 \mathrm{C})$ & 109.5 \\
\hline $\mathrm{C}(70)-\mathrm{C}(69)-\mathrm{N}(1)$ & $115.2(3)$ & & \\
\hline
\end{tabular}


Table 9. Anisotropic displacement parameters $\left(\AA^{2} \times 10^{3}\right)$ for $\left\{\left[\mathrm{NEt}_{4}\right]\left[\mathrm{Ph}_{3} \mathrm{BP}^{m-\mathrm{Pr} 2}\right]\right\}_{2}$. The anisotropic displacement factor exponent takes the form: $-2 \pi^{2}\left[\mathrm{~h}^{2} \mathrm{a}^{* 2} \mathrm{U}^{11}+\ldots+2 \mathrm{~h} \mathrm{k} \mathrm{a}^{*} \mathrm{~b}^{*}\right.$ $\left.\mathrm{U}^{12}\right]$.

\begin{tabular}{|c|c|c|c|c|c|c|}
\hline & $\mathrm{U}^{11}$ & $\mathrm{U}^{22}$ & $\mathrm{U}^{33}$ & $\mathrm{U}^{23}$ & $\mathrm{U}^{13}$ & $\mathrm{U}^{12}$ \\
\hline$\overline{B(1)}$ & $24(2)$ & $24(2)$ & $21(2)$ & 1(2) & $3(2)$ & $6(2)$ \\
\hline $\mathrm{P}(1)$ & $26(1)$ & $22(1)$ & $38(1)$ & $1(1)$ & $1(1)$ & 1(1) \\
\hline $\mathrm{C}(1)$ & $19(2)$ & $19(2)$ & $23(2)$ & $3(2)$ & $0(1)$ & $3(1)$ \\
\hline$C(2)$ & $26(2)$ & $19(2)$ & $27(2)$ & $-2(2)$ & $6(2)$ & $3(1)$ \\
\hline $\mathrm{C}(3)$ & $39(2)$ & $26(2)$ & $20(2)$ & $1(2)$ & $5(2)$ & $-4(2)$ \\
\hline$C(4)$ & $48(2)$ & $34(2)$ & $26(2)$ & $5(2)$ & $1(2)$ & $-4(2)$ \\
\hline$C(5)$ & $58(2)$ & $20(2)$ & $31(2)$ & $8(2)$ & $-12(2)$ & $-6(2)$ \\
\hline$C(6)$ & $44(2)$ & $22(2)$ & $27(2)$ & $-3(2)$ & $-10(2)$ & $-1(2)$ \\
\hline$C(7)$ & $29(2)$ & $19(2)$ & $22(2)$ & $-5(2)$ & $-6(2)$ & $2(2)$ \\
\hline $\mathrm{C}(8)$ & $24(2)$ & $33(2)$ & $39(2)$ & $-8(2)$ & $5(2)$ & $3(2)$ \\
\hline $\mathrm{C}(9)$ & $30(2)$ & $34(2)$ & $48(2)$ & $-9(2)$ & $1(2)$ & $7(2)$ \\
\hline$C(10)$ & $23(2)$ & $52(3)$ & $36(2)$ & $-15(2)$ & $12(2)$ & $6(2)$ \\
\hline$C(11)$ & $32(2)$ & $44(2)$ & $34(2)$ & $6(2)$ & $14(2)$ & $3(2)$ \\
\hline$C(12)$ & $30(2)$ & $34(2)$ & $29(2)$ & $3(2)$ & $9(2)$ & $5(2)$ \\
\hline$C(13)$ & $22(2)$ & $13(2)$ & $23(2)$ & $-3(1)$ & $0(1)$ & $7(1)$ \\
\hline$C(14)$ & $36(2)$ & $21(2)$ & $29(2)$ & $6(2)$ & $2(2)$ & $1(2)$ \\
\hline$C(15)$ & $48(2)$ & $33(2)$ & $17(2)$ & $1(2)$ & $-5(2)$ & $1(2)$ \\
\hline$C(16)$ & $34(2)$ & $24(2)$ & $37(2)$ & $-1(2)$ & $-18(2)$ & $3(2)$ \\
\hline$C(17)$ & $21(2)$ & $18(2)$ & $43(2)$ & $-2(2)$ & $-5(2)$ & $5(1)$ \\
\hline $\mathrm{C}(18)$ & $29(2)$ & $21(2)$ & $27(2)$ & $-2(2)$ & $-1(2)$ & $5(2)$ \\
\hline$C(19)$ & $20(2)$ & $15(2)$ & $19(2)$ & $-1(1)$ & 1(1) & $3(1)$ \\
\hline$C(20)$ & $15(2)$ & $24(2)$ & $22(2)$ & $3(2)$ & $2(1)$ & $3(1)$ \\
\hline$C(21)$ & $22(2)$ & $17(2)$ & $20(2)$ & $2(1)$ & $0(1)$ & $3(1)$ \\
\hline$C(22)$ & $31(2)$ & $19(2)$ & $33(2)$ & $-2(2)$ & $0(2)$ & $5(2)$ \\
\hline $\mathrm{C}(23)$ & $28(2)$ & $30(2)$ & $44(2)$ & $-6(2)$ & $-13(2)$ & $12(2)$ \\
\hline$C(24)$ & $33(2)$ & $23(2)$ & $37(2)$ & $-1(2)$ & $-6(2)$ & $-1(2)$ \\
\hline$C(25)$ & $39(2)$ & $21(2)$ & $33(2)$ & $-5(2)$ & $-7(2)$ & $1(2)$ \\
\hline$C(26)$ & $48(2)$ & $32(2)$ & $32(2)$ & $-4(2)$ & $-6(2)$ & $3(2)$ \\
\hline$C(27)$ & $46(2)$ & $23(2)$ & $30(2)$ & $-1(2)$ & $2(2)$ & $0(2)$ \\
\hline$C(28)$ & $30(2)$ & $18(2)$ & $39(2)$ & $1(2)$ & $6(2)$ & $2(2)$ \\
\hline$C(29)$ & $39(2)$ & $29(2)$ & $43(2)$ & $-3(2)$ & $9(2)$ & $2(2)$ \\
\hline$C(30)$ & $43(2)$ & $31(2)$ & $42(2)$ & $5(2)$ & $17(2)$ & $-1(2)$ \\
\hline $\mathrm{B}(2)$ & $23(2)$ & $21(2)$ & $22(2)$ & $-4(2)$ & $-3(2)$ & $2(2)$ \\
\hline $\mathrm{P}(2)$ & $24(1)$ & 21(1) & $34(1)$ & $-5(1)$ & $-1(1)$ & $3(1)$ \\
\hline$C(31)$ & $18(2)$ & $20(2)$ & $25(2)$ & $0(2)$ & $2(1)$ & $-3(1)$ \\
\hline$C(32)$ & $37(2)$ & $25(2)$ & $30(2)$ & $9(2)$ & $9(2)$ & $6(2)$ \\
\hline$C(33)$ & $54(2)$ & $39(3)$ & $21(2)$ & $1(2)$ & $9(2)$ & $9(2)$ \\
\hline$C(34)$ & $47(2)$ & 43(3) & $29(2)$ & $13(2)$ & $5(2)$ & $1(2)$ \\
\hline$C(35)$ & $55(2)$ & $21(2)$ & $34(2)$ & $6(2)$ & $-9(2)$ & $0(2)$ \\
\hline$C(36)$ & $49(2)$ & $22(2)$ & $26(2)$ & $2(2)$ & $-5(2)$ & $-5(2)$ \\
\hline$C(37)$ & $24(2)$ & $16(2)$ & $21(2)$ & $6(1)$ & $0(1)$ & $5(1)$ \\
\hline$C(38)$ & $30(2)$ & $22(2)$ & $24(2)$ & $-2(2)$ & $0(2)$ & 1(2) \\
\hline$C(39)$ & $26(2)$ & $25(2)$ & $44(2)$ & $2(2)$ & $-2(2)$ & $0(2)$ \\
\hline$C(40)$ & $37(2)$ & $19(2)$ & $40(2)$ & $-11(2)$ & $-17(2)$ & 1(2) \\
\hline$C(41)$ & $49(2)$ & $39(3)$ & $26(2)$ & $-4(2)$ & $-8(2)$ & $11(2)$ \\
\hline$C(42)$ & $30(2)$ & $27(2)$ & $26(2)$ & $-3(2)$ & $-1(2)$ & $1(2)$ \\
\hline
\end{tabular}




$\begin{array}{llllccc}\mathrm{C}(43) & 23(2) & 25(2) & 16(2) & -3(2) & -1(1) & 1(2) \\ \mathrm{C}(44) & 29(2) & 17(2) & 55(2) & -2(2) & 7(2) & -4(2) \\ \mathrm{C}(45) & 29(2) & 27(2) & 59(3) & -2(2) & 8(2) & 9(2) \\ \mathrm{C}(46) & 24(2) & 37(2) & 36(2) & -11(2) & 8(2) & -2(2) \\ \mathrm{C}(47) & 24(2) & 27(2) & 22(2) & 2(2) & 2(1) & -5(2) \\ \mathrm{C}(48) & 28(2) & 20(2) & 18(2) & -2(1) & 3(1) & 2(1) \\ \mathrm{C}(49) & 21(2) & 26(2) & 12(2) & 6(1) & 3(1) & -3(1) \\ \mathrm{C}(50) & 21(2) & 19(2) & 14(2) & 3(1) & 1(1) & 4(1) \\ \mathrm{C}(51) & 21(2) & 28(2) & 17(2) & 4(2) & 5(1) & 2(2) \\ \mathrm{C}(52) & 23(2) & 20(2) & 22(2) & 2(2) & 3(1) & 4(1) \\ \mathrm{C}(53) & 19(2) & 34(2) & 18(2) & 4(2) & 0(1) & -1(2) \\ \mathrm{C}(54) & 28(2) & 17(2) & 17(2) & -1(1) & 0(1) & -1(1) \\ \mathrm{C}(55) & 35(2) & 22(2) & 25(2) & -4(2) & -10(1) & 5(2) \\ \mathrm{C}(56) & 51(2) & 31(2) & 29(2) & -2(2) & -11(2) & 10(2) \\ \mathrm{C}(57) & 45(2) & 39(2) & 19(2) & -2(2) & 2(2) & 5(2) \\ \mathrm{C}(58) & 27(2) & 22(2) & 36(2) & -2(2) & 11(2) & 1(2) \\ \mathrm{C}(59) & 42(2) & 22(2) & 55(3) & -6(2) & 11(2) & 0(2) \\ \mathrm{C}(60) & 32(2) & 30(2) & 40(2) & 2(2) & 8(2) & 5(2) \\ \mathrm{N}(1) & 28(2) & 31(2) & 30(2) & 5(1) & 8(1) & 0(1) \\ \mathrm{C}(61) & 64(3) & 40(3) & 47(3) & 1(2) & 11(2) & 3(2) \\ \mathrm{C}(62) & 46(2) & 36(2) & 94(3) & -25(2) & -17(2) & 4(2) \\ \mathrm{C}(63) & 49(2) & 53(3) & 45(3) & -10(2) & 5(2) & -8(2) \\ \mathrm{C}(64) & 86(3) & 39(3) & 36(2) & 17(2) & 7(2) & 1(2) \\ \mathrm{C}(65) & 37(2) & 60(3) & 63(3) & 1(2) & -2(2) & 7(2) \\ \mathrm{C}(66) & 39(2) & 35(2) & 75(3) & -31(2) & -6(2) & 8(2) \\ \mathrm{C}(67) & 50(2) & 44(3) & 51(3) & 0(2) & 2(2) & 3(2) \\ \mathrm{C}(68) & 71(3) & 85(3) & 45(3) & 22(2) & 22(2) & 37(3) \\ \mathrm{N}(2) & 26(2) & 25(2) & 25(2) & -6(1) & 5(1) & -3(1) \\ \mathrm{C}(69) & 30(2) & 31(2) & 53(2) & -5(2) & 11(2) & -9(2) \\ \mathrm{C}(70) & 55(3) & 52(3) & 61(3) & -12(2) & -23(2) & 2(2) \\ \mathrm{C}(71) & 36(2) & 36(2) & 59(3) & -9(2) & 20(2) & 4(2) \\ \mathrm{C}(72) & 43(2) & 32(2) & 100(4) & -4(2) & 15(2) & 4(2) \\ \mathrm{C}(73) & 42(2) & 35(2) & 41(2) & 5(2) & -14(2) & 9(2) \\ \mathrm{C}(74) & 47(2) & 42(3) & 107(4) & -9(2) & -31(2) & 15(2) \\ \mathrm{C}(75) & 47(2) & 38(2) & 31(2) & 1(2) & 0(2) & 4(2) \\ \mathrm{C}(76) & 56(2) & 34(2) & 35(2) & 6(2) & -12(2) & 2(2) \\ & & & & & & \end{array}$

Table 10. Hydrogen coordinates $\left(\times 10^{4}\right)$ and isotropic displacement parameters $\left(\AA^{2} \times 10^{3}\right)$ for $\left\{\left[\mathrm{NEt}_{4}\right]\left[\mathrm{Ph}_{3} \mathrm{BP}^{m-\mathrm{Pr} 2}\right]\right\}_{2}$. 


\begin{tabular}{|c|c|c|c|c|}
\hline & $\mathrm{x}$ & $\mathrm{y}$ & $\mathrm{z}$ & $\mathrm{U}(\mathrm{eq})$ \\
\hline $\mathrm{H}(2)$ & 449 & 8088 & 3734 & 29 \\
\hline $\mathrm{H}(3)$ & 755 & 7765 & 5051 & 34 \\
\hline $\mathrm{H}(4)$ & 768 & 6637 & 5383 & 43 \\
\hline $\mathrm{H}(5)$ & 446 & 5855 & 4373 & 44 \\
\hline $\mathrm{H}(6)$ & 174 & 6189 & 3056 & 38 \\
\hline $\mathrm{H}(8)$ & 2161 & 6474 & 2325 & 38 \\
\hline $\mathrm{H}(9)$ & 4363 & 6435 & 1950 & 45 \\
\hline $\mathrm{H}(10)$ & 5329 & 7353 & 1324 & 44 \\
\hline $\mathrm{H}(11)$ & 4008 & 8307 & 1100 & 44 \\
\hline $\mathrm{H}(12)$ & 1796 & 8329 & 1474 & 37 \\
\hline $\mathrm{H}(14)$ & 184 & 7003 & 707 & 34 \\
\hline $\mathrm{H}(15)$ & -1291 & 6346 & -110 & 39 \\
\hline $\mathrm{H}(16)$ & -3086 & 5806 & 442 & 39 \\
\hline $\mathrm{H}(17)$ & -3473 & 5928 & 1790 & 33 \\
\hline $\mathrm{H}(18)$ & -2074 & 6629 & 2573 & 31 \\
\hline $\mathrm{H}(20)$ & 1003 & 8714 & 2668 & 25 \\
\hline $\mathrm{H}(22)$ & -2234 & 9847 & 2064 & 33 \\
\hline $\mathrm{H}(23)$ & -3349 & 8911 & 1586 & 41 \\
\hline $\mathrm{H}(24)$ & -2327 & 7872 & 1674 & 37 \\
\hline $\mathrm{H}(25)$ & 387 & 10536 & 4130 & 38 \\
\hline $\mathrm{H}(26 \mathrm{~A})$ & 299 & 9595 & 4924 & 56 \\
\hline $\mathrm{H}(26 \mathrm{~B})$ & 1467 & 9501 & 4307 & 56 \\
\hline $\mathrm{H}(26 \mathrm{C})$ & 44 & 9131 & 4157 & 56 \\
\hline $\mathrm{H}(27 \mathrm{~A})$ & -2041 & 9863 & 3668 & 50 \\
\hline $\mathrm{H}(27 \mathrm{~B})$ & -1846 & 10649 & 3769 & 50 \\
\hline $\mathrm{H}(27 \mathrm{C})$ & -1756 & 10176 & 4536 & 50 \\
\hline $\mathrm{H}(28)$ & -1284 & 10808 & 2444 & 34 \\
\hline $\mathrm{H}(29 \mathrm{~A})$ & 1279 & 11448 & 2745 & 55 \\
\hline H(29B) & 60 & 11457 & 3337 & 55 \\
\hline $\mathrm{H}(29 \mathrm{C})$ & -89 & 11839 & 2502 & 55 \\
\hline $\mathrm{H}(30 \mathrm{~A})$ & -424 & 11221 & 1241 & 57 \\
\hline $\mathrm{H}(30 \mathrm{~B})$ & -331 & 10427 & 1242 & 57 \\
\hline $\mathrm{H}(30 \mathrm{C})$ & 997 & 10863 & 1441 & 57 \\
\hline $\mathrm{H}(32)$ & 4757 & 3331 & 1262 & 37 \\
\hline $\mathrm{H}(33)$ & 4417 & 3700 & -29 & 45 \\
\hline $\mathrm{H}(34)$ & 3855 & 4812 & -286 & 47 \\
\hline $\mathrm{H}(35)$ & 3558 & 5521 & 798 & 44 \\
\hline $\mathrm{H}(36)$ & 3835 & 5134 & 2079 & 39 \\
\hline $\mathrm{H}(38)$ & 6548 & 4735 & 2441 & 31 \\
\hline H(39) & 8012 & 5391 & 3202 & 38 \\
\hline $\mathrm{H}(40)$ & 7720 & 5516 & 4560 & 39 \\
\hline $\mathrm{H}(41)$ & 5919 & 4982 & 5127 & 46 \\
\hline $\mathrm{H}(42)$ & 4446 & 4329 & 4365 & 34 \\
\hline $\mathrm{H}(44)$ & 2567 & 4889 & 3144 & 40 \\
\hline $\mathrm{H}(45)$ & 343 & 4892 & 3492 & 46 \\
\hline $\mathrm{H}(46)$ & -817 & 3903 & 3680 & 39 \\
\hline $\mathrm{H}(47)$ & 305 & 2902 & 3527 & 29 \\
\hline $\mathrm{H}(48)$ & 2545 & 2889 & 3170 & 26 \\
\hline $\mathrm{H}(50)$ & 3790 & 2651 & 2168 & 22 \\
\hline $\mathrm{H}(52)$ & 6842 & 1493 & 3010 & 26 \\
\hline $\mathrm{H}(53)$ & 7774 & 2411 & 3687 & 28 \\
\hline $\mathrm{H}(54)$ & 6732 & 3429 & 3600 & 24 \\
\hline $\mathrm{H}(55)$ & 4719 & 923 & 694 & 33 \\
\hline
\end{tabular}




\begin{tabular}{|c|c|c|c|c|}
\hline $\mathrm{H}(56 \mathrm{~A})$ & 5219 & 2320 & 820 & 56 \\
\hline $\mathrm{H}(56 \mathrm{~B})$ & 3793 & 2007 & 524 & 56 \\
\hline $\mathrm{H}(56 \mathrm{C})$ & 5093 & 1905 & 6 & 56 \\
\hline $\mathrm{H}(57 \mathrm{~A})$ & 6982 & 1240 & 511 & 51 \\
\hline $\mathrm{H}(57 \mathrm{~B})$ & 6849 & 741 & 1245 & 51 \\
\hline $\mathrm{H}(57 \mathrm{C})$ & 7084 & 1517 & 1404 & 51 \\
\hline $\mathrm{H}(58)$ & 5993 & 557 & 2445 & 34 \\
\hline $\mathrm{H}(59 \mathrm{~A})$ & 4852 & -473 & 2233 & 59 \\
\hline $\mathrm{H}(59 \mathrm{~B})$ & 4705 & -37 & 1437 & 59 \\
\hline $\mathrm{H}(59 \mathrm{C})$ & 3465 & -91 & 2011 & 59 \\
\hline $\mathrm{H}(60 \mathrm{~A})$ & 3615 & 488 & 3357 & 50 \\
\hline $\mathrm{H}(60 \mathrm{~B})$ & 4975 & 873 & 3633 & 50 \\
\hline $\mathrm{H}(60 \mathrm{C})$ & 4976 & 80 & 3564 & 50 \\
\hline $\mathrm{H}(61 \mathrm{~A})$ & 8337 & 4716 & 1068 & 60 \\
\hline $\mathrm{H}(61 \mathrm{~B})$ & 9046 & 4387 & 1843 & 60 \\
\hline $\mathrm{H}(62 \mathrm{~A})$ & 11096 & 4889 & 1522 & 89 \\
\hline $\mathrm{H}(62 \mathrm{~B})$ & 9931 & 5415 & 1693 & 89 \\
\hline $\mathrm{H}(62 \mathrm{C})$ & 10300 & 5258 & 795 & 89 \\
\hline $\mathrm{H}(63 \mathrm{~A})$ & 10507 & 4374 & -96 & 59 \\
\hline $\mathrm{H}(63 \mathrm{~B})$ & 9784 & 3702 & -389 & 59 \\
\hline $\mathrm{H}(64 \mathrm{~A})$ & 7688 & 4248 & -392 & 81 \\
\hline $\mathrm{H}(64 \mathrm{~B})$ & 8693 & 4577 & -997 & 81 \\
\hline $\mathrm{H}(64 \mathrm{C})$ & 8465 & 4923 & -156 & 81 \\
\hline $\mathrm{H}(65 \mathrm{~A})$ & 10750 & 3569 & 1721 & 64 \\
\hline $\mathrm{H}(65 \mathrm{~B})$ & 11539 & 3940 & 1042 & 64 \\
\hline $\mathrm{H}(66 \mathrm{~A})$ & 10562 & 2603 & 965 & 75 \\
\hline $\mathrm{H}(66 \mathrm{~B})$ & 12111 & 2832 & 958 & 75 \\
\hline $\mathrm{H}(66 \mathrm{C})$ & 11107 & 2972 & 200 & 75 \\
\hline $\mathrm{H}(67 \mathrm{~A})$ & 8470 & 3083 & 443 & 58 \\
\hline $\mathrm{H}(67 \mathrm{~B})$ & 7512 & 3684 & 677 & 58 \\
\hline $\mathrm{H}(68 \mathrm{~A})$ & 9037 & 2939 & 1821 & 100 \\
\hline $\mathrm{H}(68 \mathrm{~B})$ & 7486 & 2785 & 1572 & 100 \\
\hline $\mathrm{H}(68 \mathrm{C})$ & 7891 & 3472 & 1998 & 100 \\
\hline $\mathrm{H}(69 \mathrm{~A})$ & 6382 & 2770 & 5428 & 45 \\
\hline $\mathrm{H}(69 \mathrm{~B})$ & 5337 & 3373 & 5331 & 45 \\
\hline $\mathrm{H}(70 \mathrm{~A})$ & 5272 & 3449 & 6722 & 85 \\
\hline $\mathrm{H}(70 \mathrm{~B})$ & 6779 & 3549 & 6436 & 85 \\
\hline $\mathrm{H}(70 \mathrm{C})$ & 6345 & 2855 & 6811 & 85 \\
\hline $\mathrm{H}(71 \mathrm{~A})$ & 3469 & 2844 & 6605 & 52 \\
\hline $\mathrm{H}(71 \mathrm{~B})$ & 2473 & 2477 & 5972 & 52 \\
\hline $\mathrm{H}(72 \mathrm{~A})$ & 3464 & 3796 & 5771 & 87 \\
\hline $\mathrm{H}(72 \mathrm{~B})$ & 1962 & 3616 & 6013 & 87 \\
\hline $\mathrm{H}(72 \mathrm{C})$ & 2446 & 3427 & 5146 & 87 \\
\hline $\mathrm{H}(73 \mathrm{~A})$ & 4956 & 1980 & 6653 & 48 \\
\hline $\mathrm{H}(73 \mathrm{~B})$ & 3946 & 1580 & 6057 & 48 \\
\hline $\mathrm{H}(74 \mathrm{~A})$ & 5754 & 1376 & 5229 & 100 \\
\hline $\mathrm{H}(74 \mathrm{~B})$ & 6020 & 1066 & 6100 & 100 \\
\hline $\mathrm{H}(74 \mathrm{C})$ & 6752 & 1743 & 5866 & 100 \\
\hline $\mathrm{H}(75 \mathrm{~A})$ & 3847 & 2799 & 4507 & 46 \\
\hline $\mathrm{H}(75 \mathrm{~B})$ & 4930 & 2212 & 4520 & 46 \\
\hline $\mathrm{H}(76 \mathrm{~A})$ & 3260 & 1448 & 4764 & 63 \\
\hline $\mathrm{H}(76 \mathrm{~B})$ & 2862 & 1863 & 3974 & 63 \\
\hline $\mathrm{H}(76 \mathrm{C})$ & 2164 & 2032 & 4789 & 63 \\
\hline
\end{tabular}


Table 11. Crystal data and structure refinement for $\left\{\mathrm{NBu}_{4}\right\}\left\{\left[\mathrm{Ph}_{3} \mathrm{BP}^{p-\mathrm{Pr} 2}\right] \mathrm{RhX}(\mathrm{NBD})\right\}$.

Empirical formula

Formula weight
$\mathrm{C}_{53} \mathrm{H}_{77} \mathrm{BBr}_{0.6} \mathrm{Cl}_{0.4} \mathrm{NPRh}$

988.21 
Crystal Habit

Crystal Color

Crystal size

Type of diffractometer

Wavelength

Data collection temperature

Unit cell dimensions

Volume

Z

Crystal system

Space group

Density (calculated)

$\mathrm{F}(000)$

$\theta$ range for data collection

Completeness to $\theta=28.59^{\circ}$

Index ranges

Reflections collected

Independent reflections

Absorption coefficient

Absorption correction

Structure solution program

Primary solution method

Secondary solution method

Hydrogen placement

Structure refinement program

Refinement method

Data / restraints / parameters

Goodness-of-fit on $\mathrm{F}^{2}$

Final $R$ indices $[\mathrm{I}>2 \sigma(\mathrm{I})]$

$\mathrm{R}$ indices (all data)

Type of weighting scheme used

Weighting scheme used rounded blade

orange

$0.37 \times 0.11 \times 0.22 \mathrm{~mm}^{3}$

\section{Data Collection}

CCD area detector

$0.71073 \AA$

98(2) K

$\mathrm{a}=15.8487(10) \AA$

$\alpha=90^{\circ}$

$\mathrm{b}=15.6527(10) \AA$

$\beta=102.6390(10)^{\circ}$

$\mathrm{c}=20.3787(13) \AA$

$\gamma=90^{\circ}$

4

Monoclinic

$\mathrm{P} 2{ }_{1}$

$1.331 \mathrm{Mg} / \mathrm{m}^{3}$

2076

1.66 to $28.59^{\circ}$

$94.5 \%$

$-21 \leq \mathrm{h} \leq 21,-20 \leq \mathrm{k} \leq 20,-27 \leq 1 \leq 27$

91118

$11928[\mathrm{R}(\mathrm{int})=0.0884]$

$1.278 \mathrm{~mm}^{-1}$

None

\section{Structure solution and refinement}

SHELXS-97 (Sheldrick, 1990)

direct methods

difference Fourier map

calculated positions

SHELXL-97 (Sheldrick, 1997)

Full-matrix least-squares on $\mathrm{F}^{2}$

11928 / 0 / 532

1.551

$\mathrm{R} 1=0.0432, \mathrm{wR} 2=0.0646$

$\mathrm{R} 1=0.0945, \mathrm{wR} 2=0.0705$

calc

calc $\mathrm{w}=1 /\left[{ }^{\wedge} 2^{\wedge}\left(\mathrm{Fo}^{\wedge} 2^{\wedge}\right)+(0.0000 \mathrm{P})^{\wedge} 2^{\wedge}+0.0000 \mathrm{P}\right]$ where 
$\mathrm{P}=\left(\mathrm{Fo}^{\wedge} 2^{\wedge}+2 \mathrm{Fc}^{\wedge} 2^{\wedge}\right) / 3$

Max shift/error $\quad 0.035$

Average shift/error $\quad 0.001$

Largest diff. peak and hole $\quad 0.980$ and $-0.670 \mathrm{e} \cdot \AA^{-3}$

Special Refinement Details:

Halide occupancy was modeled reasonably as $60 \%$ bromide and $40 \%$ chloride. The structure was otherwise unremarkable.

Table 12. Atomic coordinates ( $\left.\mathrm{x} 10^{4}\right)$ and equivalent isotropic displacement parameters $\left(\AA^{2} \mathrm{X}\right.$ $\left.10^{3}\right)$ for $\left\{\mathrm{NBu}_{4}\right\}\left\{\left[\mathrm{Ph}_{3} \mathrm{BP}^{p-\mathrm{Pr} 2}\right] \mathrm{RhX}(\mathrm{NBD})\right\}$. U(eq) is defined as one third of the trace of the orthogonalized Uij tensor. 


\begin{tabular}{|c|c|c|c|c|}
\hline & $\mathrm{x}$ & $\mathrm{y}$ & $\mathrm{z}$ & $\mathrm{U}(\mathrm{eq})$ \\
\hline$\overline{\mathrm{Rh}}$ & $55(1)$ & $6868(1)$ & $3120(1)$ & $16(1)$ \\
\hline $\mathrm{Br}$ & $-1523(1)$ & $6703(1)$ & $2738(1)$ & $23(1)$ \\
\hline $\mathrm{Cl}$ & $-1523(1)$ & $6703(1)$ & $2738(1)$ & $23(1)$ \\
\hline $\mathrm{P}$ & 211(1) & $7589(1)$ & $2152(1)$ & $15(1)$ \\
\hline B & $3530(2)$ & $6758(2)$ & $1001(2)$ & $15(1)$ \\
\hline $\mathrm{C}(1)$ & $3827(2)$ & $7591(2)$ & $613(1)$ & $15(1)$ \\
\hline $\mathrm{C}(2)$ & $3675(2)$ & $7666(2)$ & $-83(2)$ & $22(1)$ \\
\hline $\mathrm{C}(3)$ & $3906(2)$ & $8393(2)$ & $-398(2)$ & $26(1)$ \\
\hline $\mathrm{C}(4)$ & $4288(2)$ & $9076(2)$ & $-24(2)$ & $26(1)$ \\
\hline$C(5)$ & $4450(2)$ & $9024(2)$ & $666(2)$ & $26(1)$ \\
\hline $\mathrm{C}(6)$ & $4228(2)$ & $8294(2)$ & $975(2)$ & $22(1)$ \\
\hline$C(7)$ & $4362(2)$ & $6453(2)$ & $1600(2)$ & $17(1)$ \\
\hline $\mathrm{C}(8)$ & $5203(2)$ & $6479(2)$ & 1491(2) & $23(1)$ \\
\hline $\mathrm{C}(9)$ & $5914(2)$ & $6164(2)$ & $1959(2)$ & $28(1)$ \\
\hline $\mathrm{C}(10)$ & $5806(2)$ & $5822(2)$ & $2556(2)$ & $33(1)$ \\
\hline $\mathrm{C}(11)$ & $4996(2)$ & $5818(2)$ & $2694(2)$ & $31(1)$ \\
\hline$C(12)$ & $4283(2)$ & $6127(2)$ & $2225(2)$ & $24(1)$ \\
\hline$C(13)$ & $3271(2)$ & $5986(2)$ & $439(1)$ & $12(1)$ \\
\hline$C(14)$ & $3866(2)$ & $5416(2)$ & $272(1)$ & $14(1)$ \\
\hline$C(15)$ & $3657(2)$ & $4839(2)$ & $-257(1)$ & $18(1)$ \\
\hline$C(16)$ & $2831(2)$ & $4813(2)$ & $-647(1)$ & $18(1)$ \\
\hline$C(17)$ & $2214(2)$ & $5362(2)$ & $-495(1)$ & $18(1)$ \\
\hline $\mathrm{C}(18)$ & $2431(2)$ & $5926(2)$ & $34(1)$ & $16(1)$ \\
\hline$C(20)$ & $2219(2)$ & $6386(2)$ & $1576(1)$ & $17(1)$ \\
\hline $\mathrm{C}(21)$ & $1511(2)$ & $6564(2)$ & $1847(1)$ & $17(1)$ \\
\hline$C(22)$ & $1192(2)$ & $7396(2)$ & $1848(1)$ & $16(1)$ \\
\hline $\mathrm{C}(23)$ & $1624(2)$ & $8028(2)$ & 1561(1) & $16(1)$ \\
\hline $\mathrm{C}(24)$ & $2344(2)$ & $7832(2)$ & $1302(1)$ & $16(1)$ \\
\hline$C(25)$ & $-649(2)$ & $7341(2)$ & 1392(1) & $20(1)$ \\
\hline$C(26)$ & $-606(2)$ & $7898(2)$ & $784(1)$ & $27(1)$ \\
\hline $\mathrm{C}(27)$ & $-644(2)$ & $6404(2)$ & $1215(2)$ & $28(1)$ \\
\hline $\mathrm{C}(29)$ & $-741(2)$ & $9036(2)$ & $2352(2)$ & $22(1)$ \\
\hline$C(30)$ & $868(2)$ & $9137(2)$ & 2763(1) & $20(1)$ \\
\hline $\mathrm{C}(32)$ & $1226(2)$ & $7202(2)$ & $3794(1)$ & $18(1)$ \\
\hline $\mathrm{C}(33)$ & $1123(2)$ & $5726(2)$ & 3972(1) & $21(1)$ \\
\hline $\mathrm{C}(34)$ & $1461(2)$ & $6153(2)$ & $4658(2)$ & $23(1)$ \\
\hline$C(35)$ & $953(2)$ & $6992(2)$ & $4456(1)$ & $19(1)$ \\
\hline$C(36)$ & $39(2)$ & $6638(2)$ & $4182(1)$ & $19(1)$ \\
\hline$C(37)$ & $137(2)$ & $5864(2)$ & $3887(1)$ & $22(1)$ \\
\hline $\mathrm{N}$ & $6588(1)$ & $8278(1)$ & $3565(1)$ & $15(1)$ \\
\hline $\mathrm{C}(38)$ & $5737(2)$ & $8697(2)$ & $3610(2)$ & $17(1)$ \\
\hline C(39) & $4917(2)$ & $8245(2)$ & $3271(2)$ & $27(1)$ \\
\hline $\mathrm{C}(40)$ & $4149(2)$ & $8685(2)$ & $3464(2)$ & $27(1)$ \\
\hline $\mathrm{C}(41)$ & $3291(2)$ & $8288(3)$ & $3130(2)$ & $47(1)$ \\
\hline$C(42)$ & $6663(2)$ & $8204(2)$ & 2839(1) & $18(1)$ \\
\hline$C(43)$ & $6776(2)$ & $9044(2)$ & 2501(1) & $21(1)$ \\
\hline $\mathrm{C}(44)$ & $6774(2)$ & $8920(2)$ & $1763(1)$ & $25(1)$ \\
\hline$C(45)$ & $6927(2)$ & $9773(2)$ & $1450(2)$ & $35(1)$ \\
\hline $\mathrm{C}(46)$ & $7297(2)$ & $8831(2)$ & $3974(1)$ & $16(1)$ \\
\hline$C(47)$ & $8221(2)$ & $8518(2)$ & $4022(1)$ & $16(1)$ \\
\hline $\mathrm{C}(48)$ & $8858(2)$ & $9242(2)$ & $4241(2)$ & $20(1)$ \\
\hline
\end{tabular}




\begin{tabular}{lrrrr}
$\mathrm{C}(49)$ & $9788(2)$ & $8945(2)$ & $4410(2)$ & $23(1)$ \\
$\mathrm{C}(50)$ & $6644(2)$ & $7375(2)$ & $3836(1)$ & $19(1)$ \\
$\mathrm{C}(51)$ & $6545(2)$ & $7257(2)$ & $4555(2)$ & $27(1)$ \\
$\mathrm{C}(52)$ & $6498(2)$ & $6306(2)$ & $4710(2)$ & $31(1)$ \\
$\mathrm{C}(53)$ & $7317(2)$ & $5806(2)$ & $4677(2)$ & $43(1)$ \\
$\mathrm{C}(19)$ & $2678(2)$ & $7013(2)$ & $1304(1)$ & $13(1)$ \\
$\mathrm{C}(31)$ & $1319(2)$ & $6420(2)$ & $3489(2)$ & $22(1)$ \\
$\mathrm{C}(28)$ & $138(2)$ & $8768(2)$ & $2219(1)$ & $17(1)$ \\
\hline
\end{tabular}

Table 13. Bond lengths $[\AA]$ and angles $\left[^{\circ}\right]$ for $\left\{\mathrm{NBu}_{4}\right\}\left\{\left[\mathrm{Ph}_{3} \mathrm{BP}^{p-\mathrm{iPr} 2}\right] \mathrm{RhX}(\mathrm{NBD})\right\}$.

\begin{tabular}{llll}
\hline Rh-C(31) & $2.101(3)$ & $\mathrm{Rh}-\mathrm{C}(37)$ & $2.200(3)$ \\
$\mathrm{Rh}-\mathrm{C}(32)$ & $2.119(3)$ & $\mathrm{Rh}-\mathrm{P}$ & $2.3321(8)$ \\
$\mathrm{Rh}-\mathrm{C}(36)$ & $2.199(3)$ & $\mathrm{Rh}-\mathrm{Br}$ & $2.4653(5)$
\end{tabular}




\begin{tabular}{|c|c|c|c|}
\hline $\mathrm{P}-\mathrm{C}(22)$ & $1.819(3)$ & $\mathrm{C}(27)-\mathrm{H}(27 \mathrm{~A})$ & 0.9800 \\
\hline P-C(28) & $1.857(3)$ & $\mathrm{C}(27)-\mathrm{H}(27 \mathrm{~B})$ & 0.9800 \\
\hline $\mathrm{P}-\mathrm{C}(25)$ & $1.866(3)$ & $\mathrm{C}(27)-\mathrm{H}(27 \mathrm{C})$ & 0.9800 \\
\hline $\mathrm{B}-\mathrm{C}(1)$ & $1.647(4)$ & $C(29)-C(28)$ & $1.535(4)$ \\
\hline B-C(19) & $1.652(4)$ & $\mathrm{C}(29)-\mathrm{H}(29 \mathrm{~A})$ & 0.9800 \\
\hline B-C(13) & $1.652(4)$ & C(29)-H(29B) & 0.9800 \\
\hline $\mathrm{B}-\mathrm{C}(7)$ & $1.659(4)$ & $\mathrm{C}(29)-\mathrm{H}(29 \mathrm{C})$ & 0.9800 \\
\hline $\mathrm{C}(1)-\mathrm{C}(2)$ & $1.390(4)$ & $\mathrm{C}(30)-\mathrm{C}(28)$ & $1.529(4)$ \\
\hline$C(1)-C(6)$ & $1.397(4)$ & $\mathrm{C}(30)-\mathrm{H}(30 \mathrm{~A})$ & 0.9800 \\
\hline$C(2)-C(3)$ & $1.394(4)$ & $\mathrm{C}(30)-\mathrm{H}(30 \mathrm{~B})$ & 0.9800 \\
\hline $\mathrm{C}(2)-\mathrm{H}(2)$ & 0.9500 & $\mathrm{C}(30)-\mathrm{H}(30 \mathrm{C})$ & 0.9800 \\
\hline $\mathrm{C}(3)-\mathrm{C}(4)$ & $1.376(4)$ & $\mathrm{C}(32)-\mathrm{C}(31)$ & $1.394(4)$ \\
\hline $\mathrm{C}(3)-\mathrm{H}(3)$ & 0.9500 & $C(32)-C(35)$ & $1.540(4)$ \\
\hline$C(4)-C(5)$ & $1.374(4)$ & $\mathrm{C}(32)-\mathrm{H}(32)$ & 1.0000 \\
\hline $\mathrm{C}(4)-\mathrm{H}(4)$ & 0.9500 & $\mathrm{C}(33)-\mathrm{C}(34)$ & $1.536(4)$ \\
\hline$C(5)-C(6)$ & $1.387(4)$ & $\mathrm{C}(33)-\mathrm{C}(31)$ & $1.543(4)$ \\
\hline $\mathrm{C}(5)-\mathrm{H}(5)$ & 0.9500 & $C(33)-C(37)$ & $1.548(4)$ \\
\hline $\mathrm{C}(6)-\mathrm{H}(6)$ & 0.9500 & $\mathrm{C}(33)-\mathrm{H}(33)$ & 1.0000 \\
\hline $\mathrm{C}(7)-\mathrm{C}(8)$ & $1.399(4)$ & $\mathrm{C}(34)-\mathrm{C}(35)$ & $1.547(4)$ \\
\hline $\mathrm{C}(7)-\mathrm{C}(12)$ & $1.402(4)$ & $\mathrm{C}(34)-\mathrm{H}(34 \mathrm{~A})$ & 0.9900 \\
\hline $\mathrm{C}(8)-\mathrm{C}(9)$ & $1.397(4)$ & $\mathrm{C}(34)-\mathrm{H}(34 \mathrm{~B})$ & 0.9900 \\
\hline $\mathrm{C}(8)-\mathrm{H}(8)$ & 0.9500 & $C(35)-C(36)$ & $1.538(4)$ \\
\hline$C(9)-C(10)$ & $1.372(4)$ & $\mathrm{C}(35)-\mathrm{H}(35)$ & 1.0000 \\
\hline $\mathrm{C}(9)-\mathrm{H}(9)$ & 0.9500 & $C(36)-C(37)$ & $1.376(4)$ \\
\hline $\mathrm{C}(10)-\mathrm{C}(11)$ & $1.373(4)$ & $\mathrm{C}(36)-\mathrm{H}(36)$ & 1.0000 \\
\hline C(10)-H(10) & 0.9500 & $\mathrm{C}(37)-\mathrm{H}(37)$ & 1.0000 \\
\hline $\mathrm{C}(11)-\mathrm{C}(12)$ & $1.399(4)$ & $\mathrm{N}-\mathrm{C}(50)$ & $1.513(3)$ \\
\hline C(11)-H(11) & 0.9500 & $\mathrm{~N}-\mathrm{C}(42)$ & $1.514(3)$ \\
\hline $\mathrm{C}(12)-\mathrm{H}(12)$ & 0.9500 & $\mathrm{~N}-\mathrm{C}(46)$ & $1.515(3)$ \\
\hline $\mathrm{C}(13)-\mathrm{C}(14)$ & $1.394(4)$ & $\mathrm{N}-\mathrm{C}(38)$ & $1.521(3)$ \\
\hline $\mathrm{C}(13)-\mathrm{C}(18)$ & $1.408(4)$ & $C(38)-C(39)$ & $1.508(4)$ \\
\hline$C(14)-C(15)$ & $1.390(4)$ & $\mathrm{C}(38)-\mathrm{H}(38 \mathrm{~A})$ & 0.9900 \\
\hline C(14)-H(14) & 0.9500 & $\mathrm{C}(38)-\mathrm{H}(38 \mathrm{~B})$ & 0.9900 \\
\hline$C(15)-C(16)$ & $1.376(4)$ & $\mathrm{C}(39)-\mathrm{C}(40)$ & $1.522(4)$ \\
\hline $\mathrm{C}(15)-\mathrm{H}(15)$ & 0.9500 & $\mathrm{C}(39)-\mathrm{H}(39 \mathrm{~A})$ & 0.9900 \\
\hline$C(16)-C(17)$ & $1.387(4)$ & $\mathrm{C}(39)-\mathrm{H}(39 \mathrm{~B})$ & 0.9900 \\
\hline $\mathrm{C}(16)-\mathrm{H}(16)$ & 0.9500 & $\mathrm{C}(40)-\mathrm{C}(41)$ & $1.514(4)$ \\
\hline $\mathrm{C}(17)-\mathrm{C}(18)$ & $1.377(4)$ & $\mathrm{C}(40)-\mathrm{H}(40 \mathrm{~A})$ & 0.9900 \\
\hline $\mathrm{C}(17)-\mathrm{H}(17)$ & 0.9500 & $\mathrm{C}(40)-\mathrm{H}(40 \mathrm{~B})$ & 0.9900 \\
\hline C(18)-H(18) & 0.9500 & $\mathrm{C}(41)-\mathrm{H}(41 \mathrm{~A})$ & 0.9800 \\
\hline$C(20)-C(21)$ & $1.384(4)$ & $\mathrm{C}(41)-\mathrm{H}(41 \mathrm{~B})$ & 0.9800 \\
\hline$C(20)-C(19)$ & $1.406(4)$ & $\mathrm{C}(41)-\mathrm{H}(41 \mathrm{C})$ & 0.9800 \\
\hline C(20)-H(20) & 0.9500 & $C(42)-C(43)$ & $1.513(4)$ \\
\hline C(21)-C(22) & $1.398(4)$ & $\mathrm{C}(42)-\mathrm{H}(42 \mathrm{~A})$ & 0.9900 \\
\hline $\mathrm{C}(21)-\mathrm{H}(21)$ & 0.9500 & $\mathrm{C}(42)-\mathrm{H}(42 \mathrm{~B})$ & 0.9900 \\
\hline$C(22)-C(23)$ & $1.402(4)$ & $\mathrm{C}(43)-\mathrm{C}(44)$ & $1.516(4)$ \\
\hline$C(23)-C(24)$ & $1.391(4)$ & $\mathrm{C}(43)-\mathrm{H}(43 \mathrm{~A})$ & 0.9900 \\
\hline $\mathrm{C}(23)-\mathrm{H}(23)$ & 0.9500 & $\mathrm{C}(43)-\mathrm{H}(43 \mathrm{~B})$ & 0.9900 \\
\hline$C(24)-C(19)$ & $1.387(4)$ & $\mathrm{C}(44)-\mathrm{C}(45)$ & $1.523(4)$ \\
\hline $\mathrm{C}(24)-\mathrm{H}(24)$ & 0.9500 & $\mathrm{C}(44)-\mathrm{H}(44 \mathrm{~A})$ & 0.9900 \\
\hline$C(25)-C(27)$ & $1.512(4)$ & $\mathrm{C}(44)-\mathrm{H}(44 \mathrm{~B})$ & 0.9900 \\
\hline C(25)-C(26) & $1.529(4)$ & $\mathrm{C}(45)-\mathrm{H}(45 \mathrm{~A})$ & 0.9800 \\
\hline $\mathrm{C}(25)-\mathrm{H}(25)$ & 1.0000 & $\mathrm{C}(45)-\mathrm{H}(45 \mathrm{~B})$ & 0.9800 \\
\hline C(26)-H(26A) & 0.9800 & $\mathrm{C}(45)-\mathrm{H}(45 \mathrm{C})$ & 0.9800 \\
\hline $\mathrm{C}(26)-\mathrm{H}(26 \mathrm{~B})$ & 0.9800 & $\mathrm{C}(46)-\mathrm{C}(47)$ & $1.527(4)$ \\
\hline $\mathrm{C}(26)-\mathrm{H}(26 \mathrm{C})$ & 0.9800 & $\mathrm{C}(46)-\mathrm{H}(46 \mathrm{~A})$ & 0.9900 \\
\hline
\end{tabular}




\begin{tabular}{|c|c|c|c|}
\hline $\mathrm{C}(46)-\mathrm{H}(46 \mathrm{~B})$ & 0.9900 & $\mathrm{C}(1)-\mathrm{C}(2)-\mathrm{H}(2)$ & 118.8 \\
\hline $\mathrm{C}(47)-\mathrm{C}(48)$ & $1.519(4)$ & $\mathrm{C}(3)-\mathrm{C}(2)-\mathrm{H}(2)$ & 118.8 \\
\hline $\mathrm{C}(47)-\mathrm{H}(47 \mathrm{~A})$ & 0.9900 & $C(4)-C(3)-C(2)$ & $120.4(3)$ \\
\hline $\mathrm{C}(47)-\mathrm{H}(47 \mathrm{~B})$ & 0.9900 & $\mathrm{C}(4)-\mathrm{C}(3)-\mathrm{H}(3)$ & 119.8 \\
\hline $\mathrm{C}(48)-\mathrm{C}(49)$ & $1.512(4)$ & $\mathrm{C}(2)-\mathrm{C}(3)-\mathrm{H}(3)$ & 119.8 \\
\hline $\mathrm{C}(48)-\mathrm{H}(48 \mathrm{~A})$ & 0.9900 & $\mathrm{C}(5)-\mathrm{C}(4)-\mathrm{C}(3)$ & $118.8(3)$ \\
\hline $\mathrm{C}(48)-\mathrm{H}(48 \mathrm{~B})$ & 0.9900 & $\mathrm{C}(5)-\mathrm{C}(4)-\mathrm{H}(4)$ & 120.6 \\
\hline $\mathrm{C}(49)-\mathrm{H}(49 \mathrm{~A})$ & 0.9800 & $\mathrm{C}(3)-\mathrm{C}(4)-\mathrm{H}(4)$ & 120.6 \\
\hline $\mathrm{C}(49)-\mathrm{H}(49 \mathrm{~B})$ & 0.9800 & $\mathrm{C}(4)-\mathrm{C}(5)-\mathrm{C}(6)$ & $120.3(3)$ \\
\hline $\mathrm{C}(49)-\mathrm{H}(49 \mathrm{C})$ & 0.9800 & $\mathrm{C}(4)-\mathrm{C}(5)-\mathrm{H}(5)$ & 119.8 \\
\hline$C(50)-C(51)$ & $1.519(4)$ & $\mathrm{C}(6)-\mathrm{C}(5)-\mathrm{H}(5)$ & 119.8 \\
\hline $\mathrm{C}(50)-\mathrm{H}(50 \mathrm{~A})$ & 0.9900 & $\mathrm{C}(5)-\mathrm{C}(6)-\mathrm{C}(1)$ & $122.6(3)$ \\
\hline $\mathrm{C}(50)-\mathrm{H}(50 \mathrm{~B})$ & 0.9900 & $\mathrm{C}(5)-\mathrm{C}(6)-\mathrm{H}(6)$ & 118.7 \\
\hline $\mathrm{C}(51)-\mathrm{C}(52)$ & $1.526(4)$ & $\mathrm{C}(1)-\mathrm{C}(6)-\mathrm{H}(6)$ & 118.7 \\
\hline $\mathrm{C}(51)-\mathrm{H}(51 \mathrm{~A})$ & 0.9900 & $\mathrm{C}(8)-\mathrm{C}(7)-\mathrm{C}(12)$ & $115.6(3)$ \\
\hline $\mathrm{C}(51)-\mathrm{H}(51 \mathrm{~B})$ & 0.9900 & $\mathrm{C}(8)-\mathrm{C}(7)-\mathrm{B}$ & $120.4(3)$ \\
\hline $\mathrm{C}(52)-\mathrm{C}(53)$ & $1.529(4)$ & $\mathrm{C}(12)-\mathrm{C}(7)-\mathrm{B}$ & $123.9(3)$ \\
\hline $\mathrm{C}(52)-\mathrm{H}(52 \mathrm{~A})$ & 0.9900 & $C(9)-C(8)-C(7)$ & $122.4(3)$ \\
\hline $\mathrm{C}(52)-\mathrm{H}(52 \mathrm{~B})$ & 0.9900 & $\mathrm{C}(9)-\mathrm{C}(8)-\mathrm{H}(8)$ & 118.8 \\
\hline $\mathrm{C}(53)-\mathrm{H}(53 \mathrm{~A})$ & 0.9800 & $\mathrm{C}(7)-\mathrm{C}(8)-\mathrm{H}(8)$ & 118.8 \\
\hline $\mathrm{C}(53)-\mathrm{H}(53 \mathrm{~B})$ & 0.9800 & $\mathrm{C}(10)-\mathrm{C}(9)-\mathrm{C}(8)$ & $120.3(3)$ \\
\hline $\mathrm{C}(53)-\mathrm{H}(53 \mathrm{C})$ & 0.9800 & $\mathrm{C}(10)-\mathrm{C}(9)-\mathrm{H}(9)$ & 119.9 \\
\hline $\mathrm{C}(31)-\mathrm{H}(31)$ & 1.0000 & $\mathrm{C}(8)-\mathrm{C}(9)-\mathrm{H}(9)$ & 119.9 \\
\hline \multirow{2}{*}{$\mathrm{C}(28)-\mathrm{H}(28)$} & 1.0000 & $\mathrm{C}(9)-\mathrm{C}(10)-\mathrm{C}(11)$ & 119.1(3) \\
\hline & & $\mathrm{C}(9)-\mathrm{C}(10)-\mathrm{H}(10)$ & 120.4 \\
\hline $\mathrm{C}(31)-\mathrm{Rh}-\mathrm{C}(32)$ & $38.59(10)$ & $\mathrm{C}(11)-\mathrm{C}(10)-\mathrm{H}(10)$ & 120.4 \\
\hline $\mathrm{C}(31)-\mathrm{Rh}-\mathrm{C}(36)$ & $78.97(11)$ & $\mathrm{C}(10)-\mathrm{C}(11)-\mathrm{C}(12)$ & $120.7(3)$ \\
\hline $\mathrm{C}(32)-\mathrm{Rh}-\mathrm{C}(36)$ & $66.40(11)$ & $\mathrm{C}(10)-\mathrm{C}(11)-\mathrm{H}(11)$ & 119.6 \\
\hline $\mathrm{C}(31)-\mathrm{Rh}-\mathrm{C}(37)$ & $66.66(12)$ & $\mathrm{C}(12)-\mathrm{C}(11)-\mathrm{H}(11)$ & 119.6 \\
\hline $\mathrm{C}(32)-\mathrm{Rh}-\mathrm{C}(37)$ & $78.94(11)$ & $\mathrm{C}(11)-\mathrm{C}(12)-\mathrm{C}(7)$ & $121.8(3)$ \\
\hline $\mathrm{C}(36)-\mathrm{Rh}-\mathrm{C}(37)$ & $36.45(10)$ & $\mathrm{C}(11)-\mathrm{C}(12)-\mathrm{H}(12)$ & 119.1 \\
\hline $\mathrm{C}(31)-\mathrm{Rh}-\mathrm{P}$ & $101.24(8)$ & $\mathrm{C}(7)-\mathrm{C}(12)-\mathrm{H}(12)$ & 119.1 \\
\hline $\mathrm{C}(32)-\mathrm{Rh}-\mathrm{P}$ & $100.90(8)$ & $\mathrm{C}(14)-\mathrm{C}(13)-\mathrm{C}(18)$ & $114.6(3)$ \\
\hline $\mathrm{C}(36)-\mathrm{Rh}-\mathrm{P}$ & $159.66(8)$ & $\mathrm{C}(14)-\mathrm{C}(13)-\mathrm{B}$ & $124.0(3)$ \\
\hline $\mathrm{C}(37)-\mathrm{Rh}-\mathrm{P}$ & $161.04(8)$ & $\mathrm{C}(18)-\mathrm{C}(13)-\mathrm{B}$ & $121.0(2)$ \\
\hline $\mathrm{C}(31)-\mathrm{Rh}-\mathrm{Br}$ & $154.23(9)$ & $\mathrm{C}(15)-\mathrm{C}(14)-\mathrm{C}(13)$ & $123.1(3)$ \\
\hline $\mathrm{C}(32)-\mathrm{Rh}-\mathrm{Br}$ & $156.22(8)$ & $\mathrm{C}(15)-\mathrm{C}(14)-\mathrm{H}(14)$ & 118.4 \\
\hline $\mathrm{C}(36)-\mathrm{Rh}-\mathrm{Br}$ & $93.69(8)$ & $\mathrm{C}(13)-\mathrm{C}(14)-\mathrm{H}(14)$ & 118.4 \\
\hline $\mathrm{C}(37)-\mathrm{Rh}-\mathrm{Br}$ & $92.75(8)$ & $\mathrm{C}(16)-\mathrm{C}(15)-\mathrm{C}(14)$ & $120.2(3)$ \\
\hline $\mathrm{P}-\mathrm{Rh}-\mathrm{Br}$ & $94.10(2)$ & $\mathrm{C}(16)-\mathrm{C}(15)-\mathrm{H}(15)$ & 119.9 \\
\hline $\mathrm{C}(22)-\mathrm{P}-\mathrm{C}(28)$ & $105.14(14)$ & $\mathrm{C}(14)-\mathrm{C}(15)-\mathrm{H}(15)$ & 119.9 \\
\hline $\mathrm{C}(22)-\mathrm{P}-\mathrm{C}(25)$ & $101.92(13)$ & $\mathrm{C}(15)-\mathrm{C}(16)-\mathrm{C}(17)$ & $118.7(3)$ \\
\hline C(28)-P-C(25) & $102.78(13)$ & $\mathrm{C}(15)-\mathrm{C}(16)-\mathrm{H}(16)$ & 120.6 \\
\hline$C(22)-P-R h$ & $117.69(10)$ & $\mathrm{C}(17)-\mathrm{C}(16)-\mathrm{H}(16)$ & 120.6 \\
\hline $\mathrm{C}(28)-\mathrm{P}-\mathrm{Rh}$ & $113.52(10)$ & $\mathrm{C}(18)-\mathrm{C}(17)-\mathrm{C}(16)$ & $120.3(3)$ \\
\hline $\mathrm{C}(25)-\mathrm{P}-\mathrm{Rh}$ & $114.07(10)$ & $\mathrm{C}(18)-\mathrm{C}(17)-\mathrm{H}(17)$ & 119.8 \\
\hline $\mathrm{C}(1)-\mathrm{B}-\mathrm{C}(19)$ & $109.3(2)$ & $\mathrm{C}(16)-\mathrm{C}(17)-\mathrm{H}(17)$ & 119.8 \\
\hline C(1)-B-C(13) & $107.5(2)$ & $\mathrm{C}(17)-\mathrm{C}(18)-\mathrm{C}(13)$ & $123.0(3)$ \\
\hline $\mathrm{C}(19)-\mathrm{B}-\mathrm{C}(13)$ & $109.7(2)$ & $\mathrm{C}(17)-\mathrm{C}(18)-\mathrm{H}(18)$ & 118.5 \\
\hline C(1)-B-C(7) & $108.5(2)$ & $\mathrm{C}(13)-\mathrm{C}(18)-\mathrm{H}(18)$ & 118.5 \\
\hline $\mathrm{C}(19)-\mathrm{B}-\mathrm{C}(7)$ & $112.2(2)$ & $\mathrm{C}(21)-\mathrm{C}(20)-\mathrm{C}(19)$ & $123.5(3)$ \\
\hline $\mathrm{C}(13)-\mathrm{B}-\mathrm{C}(7)$ & $109.7(2)$ & $\mathrm{C}(21)-\mathrm{C}(20)-\mathrm{H}(20)$ & 118.3 \\
\hline$C(2)-C(1)-C(6)$ & $115.5(3)$ & $\mathrm{C}(19)-\mathrm{C}(20)-\mathrm{H}(20)$ & 118.3 \\
\hline $\mathrm{C}(2)-\mathrm{C}(1)-\mathrm{B}$ & $123.5(3)$ & $\mathrm{C}(20)-\mathrm{C}(21)-\mathrm{C}(22)$ & $120.9(3)$ \\
\hline$C(6)-C(1)-B$ & $121.0(3)$ & $\mathrm{C}(20)-\mathrm{C}(21)-\mathrm{H}(21)$ & 119.5 \\
\hline $\mathrm{C}(1)-\mathrm{C}(2)-\mathrm{C}(3)$ & $122.3(3)$ & $\mathrm{C}(22)-\mathrm{C}(21)-\mathrm{H}(21)$ & 119.5 \\
\hline
\end{tabular}




\begin{tabular}{|c|c|c|c|}
\hline$C(21)-C(22)-C(23)$ & $116.5(3)$ & $\mathrm{H}(34 \mathrm{~A})-\mathrm{C}(34)-\mathrm{H}(34 \mathrm{~B})$ & 110.3 \\
\hline $\mathrm{C}(21)-\mathrm{C}(22)-\mathrm{P}$ & $119.4(2)$ & $\mathrm{C}(36)-\mathrm{C}(35)-\mathrm{C}(32)$ & $100.4(2)$ \\
\hline$C(23)-C(22)-P$ & $123.9(2)$ & $C(36)-C(35)-C(34)$ & $100.7(2)$ \\
\hline$C(24)-C(23)-C(22)$ & 121.3(3) & $\mathrm{C}(32)-\mathrm{C}(35)-\mathrm{C}(34)$ & $100.6(2)$ \\
\hline $\mathrm{C}(24)-\mathrm{C}(23)-\mathrm{H}(23)$ & 119.4 & $\mathrm{C}(36)-\mathrm{C}(35)-\mathrm{H}(35)$ & 117.3 \\
\hline $\mathrm{C}(22)-\mathrm{C}(23)-\mathrm{H}(23)$ & 119.4 & $\mathrm{C}(32)-\mathrm{C}(35)-\mathrm{H}(35)$ & 117.3 \\
\hline $\mathrm{C}(19)-\mathrm{C}(24)-\mathrm{C}(23)$ & 123.1(3) & $\mathrm{C}(34)-\mathrm{C}(35)-\mathrm{H}(35)$ & 117.3 \\
\hline $\mathrm{C}(19)-\mathrm{C}(24)-\mathrm{H}(24)$ & 118.4 & $\mathrm{C}(37)-\mathrm{C}(36)-\mathrm{C}(35)$ & 106.7(3) \\
\hline $\mathrm{C}(23)-\mathrm{C}(24)-\mathrm{H}(24)$ & 118.4 & $\mathrm{C}(37)-\mathrm{C}(36)-\mathrm{Rh}$ & $71.82(16)$ \\
\hline$C(27)-C(25)-C(26)$ & $110.8(2)$ & $C(35)-C(36)-R h$ & $94.93(17)$ \\
\hline$C(27)-C(25)-P$ & $111.0(2)$ & $\mathrm{C}(37)-\mathrm{C}(36)-\mathrm{H}(36)$ & 123.3 \\
\hline$C(26)-C(25)-P$ & $113.6(2)$ & $\mathrm{C}(35)-\mathrm{C}(36)-\mathrm{H}(36)$ & 123.3 \\
\hline $\mathrm{C}(27)-\mathrm{C}(25)-\mathrm{H}(25)$ & 107.0 & Rh-C(36)-H(36) & 123.3 \\
\hline $\mathrm{C}(26)-\mathrm{C}(25)-\mathrm{H}(25)$ & 107.0 & $\mathrm{C}(36)-\mathrm{C}(37)-\mathrm{C}(33)$ & $106.4(3)$ \\
\hline $\mathrm{P}-\mathrm{C}(25)-\mathrm{H}(25)$ & 107.0 & $C(36)-C(37)-R h$ & $71.72(17)$ \\
\hline $\mathrm{C}(25)-\mathrm{C}(26)-\mathrm{H}(26 \mathrm{~A})$ & 109.5 & $C(33)-C(37)-R h$ & $94.60(17)$ \\
\hline $\mathrm{C}(25)-\mathrm{C}(26)-\mathrm{H}(26 \mathrm{~B})$ & 109.5 & $\mathrm{C}(36)-\mathrm{C}(37)-\mathrm{H}(37)$ & 123.5 \\
\hline $\mathrm{H}(26 \mathrm{~A})-\mathrm{C}(26)-\mathrm{H}(26 \mathrm{~B})$ & 109.5 & $\mathrm{C}(33)-\mathrm{C}(37)-\mathrm{H}(37)$ & 123.5 \\
\hline $\mathrm{C}(25)-\mathrm{C}(26)-\mathrm{H}(26 \mathrm{C})$ & 109.5 & Rh-C(37)-H(37) & 123.5 \\
\hline $\mathrm{H}(26 \mathrm{~A})-\mathrm{C}(26)-\mathrm{H}(26 \mathrm{C})$ & 109.5 & $\mathrm{C}(50)-\mathrm{N}-\mathrm{C}(42)$ & $105.9(2)$ \\
\hline $\mathrm{H}(26 \mathrm{~B})-\mathrm{C}(26)-\mathrm{H}(26 \mathrm{C})$ & 109.5 & $\mathrm{C}(50)-\mathrm{N}-\mathrm{C}(46)$ & $110.7(2)$ \\
\hline $\mathrm{C}(25)-\mathrm{C}(27)-\mathrm{H}(27 \mathrm{~A})$ & 109.5 & $\mathrm{C}(42)-\mathrm{N}-\mathrm{C}(46)$ & $112.0(2)$ \\
\hline $\mathrm{C}(25)-\mathrm{C}(27)-\mathrm{H}(27 \mathrm{~B})$ & 109.5 & $\mathrm{C}(50)-\mathrm{N}-\mathrm{C}(38)$ & 111.2(2) \\
\hline $\mathrm{H}(27 \mathrm{~A})-\mathrm{C}(27)-\mathrm{H}(27 \mathrm{~B})$ & 109.5 & $\mathrm{C}(42)-\mathrm{N}-\mathrm{C}(38)$ & $110.7(2)$ \\
\hline $\mathrm{C}(25)-\mathrm{C}(27)-\mathrm{H}(27 \mathrm{C})$ & 109.5 & $\mathrm{C}(46)-\mathrm{N}-\mathrm{C}(38)$ & $106.3(2)$ \\
\hline $\mathrm{H}(27 \mathrm{~A})-\mathrm{C}(27)-\mathrm{H}(27 \mathrm{C})$ & 109.5 & $\mathrm{C}(39)-\mathrm{C}(38)-\mathrm{N}$ & $117.2(2)$ \\
\hline $\mathrm{H}(27 \mathrm{~B})-\mathrm{C}(27)-\mathrm{H}(27 \mathrm{C})$ & 109.5 & $\mathrm{C}(39)-\mathrm{C}(38)-\mathrm{H}(38 \mathrm{~A})$ & 108.0 \\
\hline $\mathrm{C}(28)-\mathrm{C}(29)-\mathrm{H}(29 \mathrm{~A})$ & 109.5 & $\mathrm{~N}-\mathrm{C}(38)-\mathrm{H}(38 \mathrm{~A})$ & 108.0 \\
\hline $\mathrm{C}(28)-\mathrm{C}(29)-\mathrm{H}(29 \mathrm{~B})$ & 109.5 & $\mathrm{C}(39)-\mathrm{C}(38)-\mathrm{H}(38 \mathrm{~B})$ & 108.0 \\
\hline $\mathrm{H}(29 \mathrm{~A})-\mathrm{C}(29)-\mathrm{H}(29 \mathrm{~B})$ & 109.5 & $\mathrm{~N}-\mathrm{C}(38)-\mathrm{H}(38 \mathrm{~B})$ & 108.0 \\
\hline $\mathrm{C}(28)-\mathrm{C}(29)-\mathrm{H}(29 \mathrm{C})$ & 109.5 & H(38A)-C(38)-H(38B) & 107.2 \\
\hline $\mathrm{H}(29 \mathrm{~A})-\mathrm{C}(29)-\mathrm{H}(29 \mathrm{C})$ & 109.5 & $\mathrm{C}(38)-\mathrm{C}(39)-\mathrm{C}(40)$ & 109.1(3) \\
\hline $\mathrm{H}(29 \mathrm{~B})-\mathrm{C}(29)-\mathrm{H}(29 \mathrm{C})$ & 109.5 & $\mathrm{C}(38)-\mathrm{C}(39)-\mathrm{H}(39 \mathrm{~A})$ & 109.9 \\
\hline $\mathrm{C}(28)-\mathrm{C}(30)-\mathrm{H}(30 \mathrm{~A})$ & 109.5 & $\mathrm{C}(40)-\mathrm{C}(39)-\mathrm{H}(39 \mathrm{~A})$ & 109.9 \\
\hline $\mathrm{C}(28)-\mathrm{C}(30)-\mathrm{H}(30 \mathrm{~B})$ & 109.5 & C(38)-C(39)-H(39B) & 109.9 \\
\hline $\mathrm{H}(30 \mathrm{~A})-\mathrm{C}(30)-\mathrm{H}(30 \mathrm{~B})$ & 109.5 & $\mathrm{C}(40)-\mathrm{C}(39)-\mathrm{H}(39 \mathrm{~B})$ & 109.9 \\
\hline C(28)-C(30)-H(30C) & 109.5 & H(39A)-C(39)-H(39B) & 108.3 \\
\hline $\mathrm{H}(30 \mathrm{~A})-\mathrm{C}(30)-\mathrm{H}(30 \mathrm{C})$ & 109.5 & $\mathrm{C}(41)-\mathrm{C}(40)-\mathrm{C}(39)$ & $112.9(3)$ \\
\hline $\mathrm{H}(30 \mathrm{~B})-\mathrm{C}(30)-\mathrm{H}(30 \mathrm{C})$ & 109.5 & $\mathrm{C}(41)-\mathrm{C}(40)-\mathrm{H}(40 \mathrm{~A})$ & 109.0 \\
\hline$C(31)-C(32)-C(35)$ & $106.3(2)$ & $\mathrm{C}(39)-\mathrm{C}(40)-\mathrm{H}(40 \mathrm{~A})$ & 109.0 \\
\hline$C(31)-C(32)-R h$ & $70.00(17)$ & $\mathrm{C}(41)-\mathrm{C}(40)-\mathrm{H}(40 \mathrm{~B})$ & 109.0 \\
\hline$C(35)-C(32)-R h$ & $98.11(18)$ & $\mathrm{C}(39)-\mathrm{C}(40)-\mathrm{H}(40 \mathrm{~B})$ & 109.0 \\
\hline $\mathrm{C}(31)-\mathrm{C}(32)-\mathrm{H}(32)$ & 123.0 & $\mathrm{H}(40 \mathrm{~A})-\mathrm{C}(40)-\mathrm{H}(40 \mathrm{~B})$ & 107.8 \\
\hline $\mathrm{C}(35)-\mathrm{C}(32)-\mathrm{H}(32)$ & 123.0 & $\mathrm{C}(40)-\mathrm{C}(41)-\mathrm{H}(41 \mathrm{~A})$ & 109.5 \\
\hline $\mathrm{Rh}-\mathrm{C}(32)-\mathrm{H}(32)$ & 123.0 & $\mathrm{C}(40)-\mathrm{C}(41)-\mathrm{H}(41 \mathrm{~B})$ & 109.5 \\
\hline$C(34)-C(33)-C(31)$ & 101.2(2) & H(41A)-C(41)-H(41B) & 109.5 \\
\hline $\mathrm{C}(34)-\mathrm{C}(33)-\mathrm{C}(37)$ & $100.6(2)$ & $\mathrm{C}(40)-\mathrm{C}(41)-\mathrm{H}(41 \mathrm{C})$ & 109.5 \\
\hline $\mathrm{C}(31)-\mathrm{C}(33)-\mathrm{C}(37)$ & $99.8(2)$ & $\mathrm{H}(41 \mathrm{~A})-\mathrm{C}(41)-\mathrm{H}(41 \mathrm{C})$ & 109.5 \\
\hline $\mathrm{C}(34)-\mathrm{C}(33)-\mathrm{H}(33)$ & 117.4 & H(41B)-C(41)-H(41C) & 109.5 \\
\hline $\mathrm{C}(31)-\mathrm{C}(33)-\mathrm{H}(33)$ & 117.4 & $\mathrm{C}(43)-\mathrm{C}(42)-\mathrm{N}$ & $114.8(2)$ \\
\hline $\mathrm{C}(37)-\mathrm{C}(33)-\mathrm{H}(33)$ & 117.4 & $\mathrm{C}(43)-\mathrm{C}(42)-\mathrm{H}(42 \mathrm{~A})$ & 108.6 \\
\hline $\mathrm{C}(33)-\mathrm{C}(34)-\mathrm{C}(35)$ & $94.0(2)$ & $\mathrm{N}-\mathrm{C}(42)-\mathrm{H}(42 \mathrm{~A})$ & 108.6 \\
\hline $\mathrm{C}(33)-\mathrm{C}(34)-\mathrm{H}(34 \mathrm{~A})$ & 112.9 & $\mathrm{C}(43)-\mathrm{C}(42)-\mathrm{H}(42 \mathrm{~B})$ & 108.6 \\
\hline $\mathrm{C}(35)-\mathrm{C}(34)-\mathrm{H}(34 \mathrm{~A})$ & 112.9 & $\mathrm{~N}-\mathrm{C}(42)-\mathrm{H}(42 \mathrm{~B})$ & 108.6 \\
\hline $\mathrm{C}(33)-\mathrm{C}(34)-\mathrm{H}(34 \mathrm{~B})$ & 112.9 & $\mathrm{H}(42 \mathrm{~A})-\mathrm{C}(42)-\mathrm{H}(42 \mathrm{~B})$ & 107.5 \\
\hline $\mathrm{C}(35)-\mathrm{C}(34)-\mathrm{H}(34 \mathrm{~B})$ & 112.9 & $C(42)-C(43)-C(44)$ & $111.4(2)$ \\
\hline
\end{tabular}




\begin{tabular}{|c|c|c|c|}
\hline $\mathrm{C}(42)-\mathrm{C}(43)-\mathrm{H}(43 \mathrm{~A})$ & 109.4 & H(49B)-C(49)-H(49C) & 109.5 \\
\hline $\mathrm{C}(44)-\mathrm{C}(43)-\mathrm{H}(43 \mathrm{~A})$ & 109.4 & $\mathrm{~N}-\mathrm{C}(50)-\mathrm{C}(51)$ & $117.2(2)$ \\
\hline $\mathrm{C}(42)-\mathrm{C}(43)-\mathrm{H}(43 \mathrm{~B})$ & 109.4 & $\mathrm{~N}-\mathrm{C}(50)-\mathrm{H}(50 \mathrm{~A})$ & 108.0 \\
\hline $\mathrm{C}(44)-\mathrm{C}(43)-\mathrm{H}(43 \mathrm{~B})$ & 109.4 & $\mathrm{C}(51)-\mathrm{C}(50)-\mathrm{H}(50 \mathrm{~A})$ & 108.0 \\
\hline $\mathrm{H}(43 \mathrm{~A})-\mathrm{C}(43)-\mathrm{H}(43 \mathrm{~B})$ & 108.0 & $\mathrm{~N}-\mathrm{C}(50)-\mathrm{H}(50 \mathrm{~B})$ & 108.0 \\
\hline $\mathrm{C}(43)-\mathrm{C}(44)-\mathrm{C}(45)$ & 109.7(3) & $\mathrm{C}(51)-\mathrm{C}(50)-\mathrm{H}(50 \mathrm{~B})$ & 108.0 \\
\hline $\mathrm{C}(43)-\mathrm{C}(44)-\mathrm{H}(44 \mathrm{~A})$ & 109.7 & $\mathrm{H}(50 \mathrm{~A})-\mathrm{C}(50)-\mathrm{H}(50 \mathrm{~B})$ & 107.3 \\
\hline $\mathrm{C}(45)-\mathrm{C}(44)-\mathrm{H}(44 \mathrm{~A})$ & 109.7 & $\mathrm{C}(50)-\mathrm{C}(51)-\mathrm{C}(52)$ & $109.8(3)$ \\
\hline $\mathrm{C}(43)-\mathrm{C}(44)-\mathrm{H}(44 \mathrm{~B})$ & 109.7 & $\mathrm{C}(50)-\mathrm{C}(51)-\mathrm{H}(51 \mathrm{~A})$ & 109.7 \\
\hline $\mathrm{C}(45)-\mathrm{C}(44)-\mathrm{H}(44 \mathrm{~B})$ & 109.7 & $\mathrm{C}(52)-\mathrm{C}(51)-\mathrm{H}(51 \mathrm{~A})$ & 109.7 \\
\hline $\mathrm{H}(44 \mathrm{~A})-\mathrm{C}(44)-\mathrm{H}(44 \mathrm{~B})$ & 108.2 & $\mathrm{C}(50)-\mathrm{C}(51)-\mathrm{H}(51 \mathrm{~B})$ & 109.7 \\
\hline $\mathrm{C}(44)-\mathrm{C}(45)-\mathrm{H}(45 \mathrm{~A})$ & 109.5 & $\mathrm{C}(52)-\mathrm{C}(51)-\mathrm{H}(51 \mathrm{~B})$ & 109.7 \\
\hline $\mathrm{C}(44)-\mathrm{C}(45)-\mathrm{H}(45 \mathrm{~B})$ & 109.5 & $\mathrm{H}(51 \mathrm{~A})-\mathrm{C}(51)-\mathrm{H}(51 \mathrm{~B})$ & 108.2 \\
\hline $\mathrm{H}(45 \mathrm{~A})-\mathrm{C}(45)-\mathrm{H}(45 \mathrm{~B})$ & 109.5 & $\mathrm{C}(51)-\mathrm{C}(52)-\mathrm{C}(53)$ & 114.2(3) \\
\hline $\mathrm{C}(44)-\mathrm{C}(45)-\mathrm{H}(45 \mathrm{C})$ & 109.5 & $\mathrm{C}(51)-\mathrm{C}(52)-\mathrm{H}(52 \mathrm{~A})$ & 108.7 \\
\hline $\mathrm{H}(45 \mathrm{~A})-\mathrm{C}(45)-\mathrm{H}(45 \mathrm{C})$ & 109.5 & $\mathrm{C}(53)-\mathrm{C}(52)-\mathrm{H}(52 \mathrm{~A})$ & 108.7 \\
\hline $\mathrm{H}(45 \mathrm{~B})-\mathrm{C}(45)-\mathrm{H}(45 \mathrm{C})$ & 109.5 & $\mathrm{C}(51)-\mathrm{C}(52)-\mathrm{H}(52 \mathrm{~B})$ & 108.7 \\
\hline $\mathrm{N}-\mathrm{C}(46)-\mathrm{C}(47)$ & $115.9(2)$ & $\mathrm{C}(53)-\mathrm{C}(52)-\mathrm{H}(52 \mathrm{~B})$ & 108.7 \\
\hline $\mathrm{N}-\mathrm{C}(46)-\mathrm{H}(46 \mathrm{~A})$ & 108.3 & $\mathrm{H}(52 \mathrm{~A})-\mathrm{C}(52)-\mathrm{H}(52 \mathrm{~B})$ & 107.6 \\
\hline $\mathrm{C}(47)-\mathrm{C}(46)-\mathrm{H}(46 \mathrm{~A})$ & 108.3 & $\mathrm{C}(52)-\mathrm{C}(53)-\mathrm{H}(53 \mathrm{~A})$ & 109.5 \\
\hline $\mathrm{N}-\mathrm{C}(46)-\mathrm{H}(46 \mathrm{~B})$ & 108.3 & $\mathrm{C}(52)-\mathrm{C}(53)-\mathrm{H}(53 \mathrm{~B})$ & 109.5 \\
\hline $\mathrm{C}(47)-\mathrm{C}(46)-\mathrm{H}(46 \mathrm{~B})$ & 108.3 & H(53A)-C(53)-H(53B) & 109.5 \\
\hline $\mathrm{H}(46 \mathrm{~A})-\mathrm{C}(46)-\mathrm{H}(46 \mathrm{~B})$ & 107.4 & $\mathrm{C}(52)-\mathrm{C}(53)-\mathrm{H}(53 \mathrm{C})$ & 109.5 \\
\hline $\mathrm{C}(48)-\mathrm{C}(47)-\mathrm{C}(46)$ & $110.2(2)$ & $\mathrm{H}(53 \mathrm{~A})-\mathrm{C}(53)-\mathrm{H}(53 \mathrm{C})$ & 109.5 \\
\hline $\mathrm{C}(48)-\mathrm{C}(47)-\mathrm{H}(47 \mathrm{~A})$ & 109.6 & $\mathrm{H}(53 \mathrm{~B})-\mathrm{C}(53)-\mathrm{H}(53 \mathrm{C})$ & 109.5 \\
\hline $\mathrm{C}(46)-\mathrm{C}(47)-\mathrm{H}(47 \mathrm{~A})$ & 109.6 & $\mathrm{C}(24)-\mathrm{C}(19)-\mathrm{C}(20)$ & $114.6(3)$ \\
\hline $\mathrm{C}(48)-\mathrm{C}(47)-\mathrm{H}(47 \mathrm{~B})$ & 109.6 & $\mathrm{C}(24)-\mathrm{C}(19)-\mathrm{B}$ & $124.5(3)$ \\
\hline $\mathrm{C}(46)-\mathrm{C}(47)-\mathrm{H}(47 \mathrm{~B})$ & 109.6 & $\mathrm{C}(20)-\mathrm{C}(19)-\mathrm{B}$ & $120.9(3)$ \\
\hline $\mathrm{H}(47 \mathrm{~A})-\mathrm{C}(47)-\mathrm{H}(47 \mathrm{~B})$ & 108.1 & $\mathrm{C}(32)-\mathrm{C}(31)-\mathrm{C}(33)$ & 106.1(3) \\
\hline$C(49)-C(48)-C(47)$ & $112.9(2)$ & $C(32)-C(31)-R h$ & $71.41(17)$ \\
\hline $\mathrm{C}(49)-\mathrm{C}(48)-\mathrm{H}(48 \mathrm{~A})$ & 109.0 & $\mathrm{C}(33)-\mathrm{C}(31)-\mathrm{Rh}$ & $98.82(19)$ \\
\hline $\mathrm{C}(47)-\mathrm{C}(48)-\mathrm{H}(48 \mathrm{~A})$ & 109.0 & $\mathrm{C}(32)-\mathrm{C}(31)-\mathrm{H}(31)$ & 122.7 \\
\hline $\mathrm{C}(49)-\mathrm{C}(48)-\mathrm{H}(48 \mathrm{~B})$ & 109.0 & $\mathrm{C}(33)-\mathrm{C}(31)-\mathrm{H}(31)$ & 122.7 \\
\hline $\mathrm{C}(47)-\mathrm{C}(48)-\mathrm{H}(48 \mathrm{~B})$ & 109.0 & $\mathrm{Rh}-\mathrm{C}(31)-\mathrm{H}(31)$ & 122.7 \\
\hline $\mathrm{H}(48 \mathrm{~A})-\mathrm{C}(48)-\mathrm{H}(48 \mathrm{~B})$ & 107.8 & $\mathrm{C}(30)-\mathrm{C}(28)-\mathrm{C}(29)$ & $109.9(2)$ \\
\hline $\mathrm{C}(48)-\mathrm{C}(49)-\mathrm{H}(49 \mathrm{~A})$ & 109.5 & $\mathrm{C}(30)-\mathrm{C}(28)-\mathrm{P}$ & $112.3(2)$ \\
\hline $\mathrm{C}(48)-\mathrm{C}(49)-\mathrm{H}(49 \mathrm{~B})$ & 109.5 & $\mathrm{C}(29)-\mathrm{C}(28)-\mathrm{P}$ & $111.0(2)$ \\
\hline $\mathrm{H}(49 \mathrm{~A})-\mathrm{C}(49)-\mathrm{H}(49 \mathrm{~B})$ & 109.5 & $\mathrm{C}(30)-\mathrm{C}(28)-\mathrm{H}(28)$ & 107.8 \\
\hline $\mathrm{C}(48)-\mathrm{C}(49)-\mathrm{H}(49 \mathrm{C})$ & 109.5 & $\mathrm{C}(29)-\mathrm{C}(28)-\mathrm{H}(28)$ & 107.8 \\
\hline $\mathrm{H}(49 \mathrm{~A})-\mathrm{C}(49)-\mathrm{H}(49 \mathrm{C})$ & 109.5 & $\mathrm{P}-\mathrm{C}(28)-\mathrm{H}(28)$ & 107.8 \\
\hline
\end{tabular}

Table 14. Anisotropic displacement parameters $\left(\AA^{2} \times 10^{3}\right)$ for $\left\{\mathrm{NBu}_{4}\right\}\left\{\mathrm{Ph}_{3} \mathrm{BP}^{p-}\right.$ $\left.\left.{ }^{\mathrm{iPr} 2}\right] \mathrm{RhX}(\mathrm{NBD})\right\}$. The anisotropic displacement factor exponent takes the form: $-2 \pi^{2}\left[\mathrm{~h}^{2}\right.$ $\left.a^{* 2} U^{11}+\ldots+2 h_{k} a^{*} b^{*} U^{12}\right]$. 


\begin{tabular}{|c|c|c|c|c|c|c|}
\hline & $\mathrm{U}^{11}$ & $\mathrm{U}^{22}$ & $\mathrm{U}^{33}$ & $\mathrm{U}^{23}$ & $\mathrm{U}^{13}$ & $\mathrm{U}^{12}$ \\
\hline$\overline{\mathrm{Rh}}$ & $16(1)$ & $18(1)$ & $15(1)$ & $0(1)$ & $5(1)$ & $1(1)$ \\
\hline $\mathrm{Br}$ & $16(1)$ & $32(1)$ & $22(1)$ & $-3(1)$ & $6(1)$ & 2(1) \\
\hline $\mathrm{Cl}$ & $16(1)$ & $32(1)$ & $22(1)$ & $-3(1)$ & $6(1)$ & 2(1) \\
\hline $\mathrm{P}$ & $16(1)$ & $16(1)$ & $14(1)$ & $-1(1)$ & $3(1)$ & 1(1) \\
\hline B & $19(2)$ & $12(2)$ & $14(2)$ & $-3(2)$ & $3(1)$ & 1(2) \\
\hline $\mathrm{C}(1)$ & $10(2)$ & $14(2)$ & $23(2)$ & $-1(1)$ & $6(1)$ & $3(1)$ \\
\hline$C(2)$ & $23(2)$ & $17(2)$ & $28(2)$ & $1(1)$ & $11(2)$ & $-1(1)$ \\
\hline$C(3)$ & $30(2)$ & $24(2)$ & $27(2)$ & $5(2)$ & $13(2)$ & $0(2)$ \\
\hline$C(4)$ & $21(2)$ & $18(2)$ & $40(2)$ & $9(2)$ & $10(2)$ & $-5(2)$ \\
\hline$C(5)$ & $21(2)$ & $18(2)$ & $37(2)$ & $-3(2)$ & $0(2)$ & $-5(1)$ \\
\hline $\mathrm{C}(6)$ & $20(2)$ & $21(2)$ & $23(2)$ & $1(1)$ & 2(1) & $-1(1)$ \\
\hline$C(7)$ & $21(2)$ & $10(2)$ & $19(2)$ & $-8(1)$ & 1(1) & 2(1) \\
\hline$C(8)$ & $24(2)$ & $18(2)$ & $26(2)$ & $-11(1)$ & $4(2)$ & $3(1)$ \\
\hline$C(9)$ & $19(2)$ & $30(2)$ & $32(2)$ & $-18(2)$ & $-1(2)$ & $3(2)$ \\
\hline $\mathrm{C}(10)$ & $32(2)$ & $23(2)$ & $33(2)$ & $-9(2)$ & $-13(2)$ & $9(2)$ \\
\hline $\mathrm{C}(11)$ & $42(2)$ & $21(2)$ & $23(2)$ & $1(2)$ & $-5(2)$ & $-4(2)$ \\
\hline $\mathrm{C}(12)$ & $26(2)$ & $15(2)$ & $27(2)$ & $-1(1)$ & $0(2)$ & $-2(1)$ \\
\hline$C(13)$ & $16(2)$ & $9(2)$ & 11(2) & $4(1)$ & $6(1)$ & $0(1)$ \\
\hline $\mathrm{C}(14)$ & $12(2)$ & $17(2)$ & $15(2)$ & $4(1)$ & $6(1)$ & $-3(1)$ \\
\hline$C(15)$ & $19(2)$ & $16(2)$ & $22(2)$ & $0(1)$ & $13(2)$ & $2(1)$ \\
\hline $\mathrm{C}(16)$ & $27(2)$ & $18(2)$ & $11(2)$ & $-3(1)$ & $6(1)$ & $-6(1)$ \\
\hline$C(17)$ & $18(2)$ & $19(2)$ & $15(2)$ & $3(1)$ & $-1(1)$ & $-2(1)$ \\
\hline$C(18)$ & $19(2)$ & $11(2)$ & $17(2)$ & $4(1)$ & $4(1)$ & $4(1)$ \\
\hline $\mathrm{C}(20)$ & $22(2)$ & $12(2)$ & $16(2)$ & $-1(1)$ & $3(1)$ & $2(1)$ \\
\hline $\mathrm{C}(21)$ & $22(2)$ & $14(2)$ & $16(2)$ & $2(1)$ & $8(1)$ & $-1(1)$ \\
\hline $\mathrm{C}(22)$ & $20(2)$ & $18(2)$ & 11(2) & $-1(1)$ & $5(1)$ & $0(1)$ \\
\hline $\mathrm{C}(23)$ & $19(2)$ & $12(2)$ & $15(2)$ & $-2(1)$ & $2(1)$ & $3(1)$ \\
\hline$C(24)$ & $19(2)$ & $16(2)$ & $14(2)$ & $2(1)$ & $3(1)$ & $-3(1)$ \\
\hline $\mathrm{C}(25)$ & $18(2)$ & $24(2)$ & $16(2)$ & $-5(1)$ & $0(1)$ & $-2(1)$ \\
\hline$C(26)$ & $31(2)$ & $30(2)$ & $16(2)$ & $-1(1)$ & $-1(2)$ & $-1(2)$ \\
\hline $\mathrm{C}(27)$ & $35(2)$ & $27(2)$ & $20(2)$ & $-7(2)$ & $5(2)$ & $-11(2)$ \\
\hline $\mathrm{C}(29)$ & $21(2)$ & $24(2)$ & $21(2)$ & $5(1)$ & $7(2)$ & $8(2)$ \\
\hline $\mathrm{C}(30)$ & $22(2)$ & $19(2)$ & $19(2)$ & $-5(1)$ & $3(1)$ & $2(1)$ \\
\hline $\mathrm{C}(32)$ & $12(2)$ & $20(2)$ & $22(2)$ & $4(1)$ & 1(1) & $3(1)$ \\
\hline $\mathrm{C}(33)$ & $28(2)$ & $17(2)$ & $20(2)$ & $4(1)$ & $7(2)$ & $6(1)$ \\
\hline $\mathrm{C}(34)$ & $25(2)$ & $23(2)$ & $20(2)$ & $4(1)$ & $4(2)$ & $5(2)$ \\
\hline $\mathrm{C}(35)$ & $23(2)$ & $19(2)$ & $16(2)$ & $-1(1)$ & $3(1)$ & $3(1)$ \\
\hline$C(36)$ & $26(2)$ & $23(2)$ & $11(2)$ & $0(1)$ & $9(1)$ & 1(1) \\
\hline $\mathrm{C}(37)$ & $30(2)$ & $18(2)$ & $17(2)$ & $7(1)$ & $7(2)$ & $-3(2)$ \\
\hline $\mathrm{N}$ & $14(1)$ & $14(1)$ & $16(1)$ & $-2(1)$ & $2(1)$ & 1(1) \\
\hline $\mathrm{C}(38)$ & $18(2)$ & $16(2)$ & $17(2)$ & $-2(1)$ & $5(1)$ & $5(1)$ \\
\hline $\mathrm{C}(39)$ & $18(2)$ & $41(2)$ & $21(2)$ & $-9(2)$ & $0(1)$ & $1(2)$ \\
\hline $\mathrm{C}(40)$ & $22(2)$ & $31(2)$ & $29(2)$ & $7(2)$ & $6(2)$ & $5(2)$ \\
\hline $\mathrm{C}(41)$ & $19(2)$ & $90(3)$ & $30(2)$ & $-10(2)$ & $-1(2)$ & $7(2)$ \\
\hline$C(42)$ & $20(2)$ & $20(2)$ & $14(2)$ & $-3(1)$ & $4(1)$ & $2(2)$ \\
\hline$C(43)$ & $25(2)$ & $20(2)$ & $17(2)$ & $3(1)$ & $3(1)$ & $3(1)$ \\
\hline $\mathrm{C}(44)$ & $23(2)$ & $31(2)$ & $19(2)$ & $2(2)$ & $1(2)$ & $-6(2)$ \\
\hline $\mathrm{C}(45)$ & $44(2)$ & $41(2)$ & $21(2)$ & $5(2)$ & $7(2)$ & $-8(2)$ \\
\hline $\mathrm{C}(46)$ & $19(2)$ & $14(2)$ & $15(2)$ & $-1(1)$ & $4(1)$ & $-3(1)$ \\
\hline$C(47)$ & $18(2)$ & $18(2)$ & $13(2)$ & 1(1) & $3(1)$ & $0(1)$ \\
\hline $\mathrm{C}(48)$ & $15(2)$ & $21(2)$ & $24(2)$ & $-4(1)$ & $6(1)$ & $-2(1)$ \\
\hline C(49) & $23(2)$ & $25(2)$ & $24(2)$ & $-6(2)$ & $7(2)$ & $-1(2)$ \\
\hline $\mathrm{C}(50)$ & $21(2)$ & $11(2)$ & $23(2)$ & 2(1) & $3(1)$ & 1(1) \\
\hline
\end{tabular}




\begin{tabular}{lllllll}
$\mathrm{C}(51)$ & $31(2)$ & $26(2)$ & $27(2)$ & $5(2)$ & $10(2)$ & $0(2)$ \\
$\mathrm{C}(52)$ & $27(2)$ & $30(2)$ & $35(2)$ & $15(2)$ & $5(2)$ & $1(2)$ \\
$\mathrm{C}(53)$ & $40(2)$ & $36(2)$ & $53(3)$ & $18(2)$ & $7(2)$ & $6(2)$ \\
$\mathrm{C}(19)$ & $14(2)$ & $15(2)$ & $10(1)$ & $-2(1)$ & $1(1)$ & $-2(1)$ \\
$\mathrm{C}(31)$ & $15(2)$ & $31(2)$ & $21(2)$ & $6(2)$ & $5(1)$ & $8(1)$ \\
$\mathrm{C}(28)$ & $16(2)$ & $18(2)$ & $15(2)$ & $1(1)$ & $3(1)$ & $2(1)$ \\
\hline
\end{tabular}

Table 15. Hydrogen coordinates (x 104) and isotropic displacement parameters $\left(\AA^{2} \times 10^{3}\right)$ for $\left\{\mathrm{NBu}_{4}\right\}\left\{\left[\mathrm{Ph}_{3} \mathrm{BP}^{p-\mathrm{Pr} 2}\right] \mathrm{RhX}(\mathrm{NBD})\right\}$.

\begin{tabular}{lllll}
\hline$x$ & $y$ & $z$ & $U(e q)$ \\
\hline
\end{tabular}




\begin{tabular}{|c|c|c|c|c|}
\hline $\mathrm{H}(2)$ & 3405 & 7206 & -353 & 26 \\
\hline $\mathrm{H}(3)$ & 3799 & 8416 & -875 & 31 \\
\hline $\mathrm{H}(4)$ & 4437 & 9576 & -237 & 31 \\
\hline $\mathrm{H}(5)$ & 4715 & 9489 & 932 & 32 \\
\hline $\mathrm{H}(6)$ & 4353 & 8270 & 1453 & 26 \\
\hline $\mathrm{H}(8)$ & 5294 & 6720 & 1084 & 28 \\
\hline $\mathrm{H}(9)$ & 6474 & 6186 & 1864 & 34 \\
\hline $\mathrm{H}(10)$ & 6286 & 5592 & 2869 & 39 \\
\hline $\mathrm{H}(11)$ & 4920 & 5602 & 3113 & 37 \\
\hline $\mathrm{H}(12)$ & 3729 & 6117 & 2332 & 28 \\
\hline $\mathrm{H}(14)$ & 4442 & 5422 & 533 & 17 \\
\hline $\mathrm{H}(15)$ & 4086 & 4461 & -349 & 21 \\
\hline $\mathrm{H}(16)$ & 2686 & 4426 & -1014 & 22 \\
\hline $\mathrm{H}(17)$ & 1639 & 5348 & -756 & 22 \\
\hline $\mathrm{H}(18)$ & 1994 & 6292 & 128 & 19 \\
\hline $\mathrm{H}(20)$ & 2405 & 5809 & 1573 & 20 \\
\hline $\mathrm{H}(21)$ & 1237 & 6114 & 2035 & 20 \\
\hline $\mathrm{H}(23)$ & 1421 & 8600 & 1542 & 19 \\
\hline $\mathrm{H}(24)$ & 2620 & 8282 & 1116 & 19 \\
\hline $\mathrm{H}(25)$ & -1217 & 7461 & 1512 & 24 \\
\hline $\mathrm{H}(26 \mathrm{~A})$ & -30 & 7851 & 685 & 40 \\
\hline $\mathrm{H}(26 \mathrm{~B})$ & -719 & 8494 & 883 & 40 \\
\hline $\mathrm{H}(26 \mathrm{C})$ & -1042 & 7705 & 394 & 40 \\
\hline $\mathrm{H}(27 \mathrm{~A})$ & -1151 & 6274 & 857 & 42 \\
\hline $\mathrm{H}(27 \mathrm{~B})$ & -664 & 6059 & 1613 & 42 \\
\hline $\mathrm{H}(27 \mathrm{C})$ & -116 & 6270 & 1060 & 42 \\
\hline $\mathrm{H}(29 \mathrm{~A})$ & -836 & 8746 & 2756 & 33 \\
\hline $\mathrm{H}(29 \mathrm{~B})$ & -1201 & 8877 & 1966 & 33 \\
\hline $\mathrm{H}(29 \mathrm{C})$ & -748 & 9656 & 2418 & 33 \\
\hline $\mathrm{H}(30 \mathrm{~A})$ & 848 & 9762 & 2742 & 31 \\
\hline $\mathrm{H}(30 \mathrm{~B})$ & 1426 & 8937 & 2690 & 31 \\
\hline $\mathrm{H}(30 \mathrm{C})$ & 797 & 8948 & 3206 & 31 \\
\hline $\mathrm{H}(32)$ & 1570 & 7720 & 3735 & 22 \\
\hline $\mathrm{H}(33)$ & 1325 & 5132 & 3911 & 25 \\
\hline $\mathrm{H}(34 \mathrm{~A})$ & 2095 & 6237 & 4763 & 27 \\
\hline $\mathrm{H}(34 \mathrm{~B})$ & 1280 & 5851 & 5031 & 27 \\
\hline $\mathrm{H}(35)$ & 1018 & 7454 & 4803 & 23 \\
\hline $\mathrm{H}(36)$ & -467 & 6779 & 4382 & 23 \\
\hline $\mathrm{H}(37)$ & -288 & 5386 & 3853 & 26 \\
\hline $\mathrm{H}(38 \mathrm{~A})$ & 5730 & 8764 & 4092 & 20 \\
\hline $\mathrm{H}(38 \mathrm{~B})$ & 5726 & 9277 & 3416 & 20 \\
\hline $\mathrm{H}(39 \mathrm{~A})$ & 4942 & 7639 & 3414 & 33 \\
\hline $\mathrm{H}(39 \mathrm{~B})$ & 4852 & 8262 & 2777 & 33 \\
\hline $\mathrm{H}(40 \mathrm{~A})$ & 4217 & 8655 & 3958 & 33 \\
\hline $\mathrm{H}(40 \mathrm{~B})$ & 4147 & 9295 & 3336 & 33 \\
\hline $\mathrm{H}(41 \mathrm{~A})$ & 3188 & 8374 & 2642 & 71 \\
\hline $\mathrm{H}(41 \mathrm{~B})$ & 2826 & 8559 & 3303 & 71 \\
\hline $\mathrm{H}(41 \mathrm{C})$ & 3303 & 7675 & 3228 & 71 \\
\hline $\mathrm{H}(42 \mathrm{~A})$ & 7161 & 7831 & 2819 & 21 \\
\hline $\mathrm{H}(42 \mathrm{~B})$ & 6136 & 7919 & 2580 & 21 \\
\hline $\mathrm{H}(43 \mathrm{~A})$ & 7329 & 9310 & 2730 & 25 \\
\hline $\mathrm{H}(43 \mathrm{~B})$ & 6301 & 9437 & 2544 & 25 \\
\hline $\mathrm{H}(44 \mathrm{~A})$ & 6211 & 8680 & 1527 & 30 \\
\hline $\mathrm{H}(44 \mathrm{~B})$ & 7234 & 8512 & 1716 & 30 \\
\hline
\end{tabular}




\begin{tabular}{lrrrl}
$\mathrm{H}(45 \mathrm{~A})$ & 6474 & 10178 & 1502 & 53 \\
$\mathrm{H}(45 \mathrm{~B})$ & 6912 & 9692 & 970 & 53 \\
$\mathrm{H}(45 \mathrm{C})$ & 7493 & 9999 & 1675 & 53 \\
$\mathrm{H}(46 \mathrm{~A})$ & 7197 & 8879 & 4436 & 19 \\
$\mathrm{H}(46 \mathrm{~B})$ & 7245 & 9411 & 3776 & 19 \\
$\mathrm{H}(47 \mathrm{~A})$ & 8349 & 8044 & 4351 & 20 \\
$\mathrm{H}(47 \mathrm{~B})$ & 8281 & 8298 & 3579 & 20 \\
$\mathrm{H}(48 \mathrm{~A})$ & 8786 & 9670 & 3876 & 24 \\
$\mathrm{H}(48 \mathrm{~B})$ & 8723 & 9524 & 4641 & 24 \\
$\mathrm{H}(49 \mathrm{~A})$ & 9878 & 8562 & 4800 & 35 \\
$\mathrm{H}(49 \mathrm{~B})$ & 10171 & 9440 & 4515 & 35 \\
$\mathrm{H}(49 \mathrm{C})$ & 9918 & 8639 & 4024 & 35 \\
$\mathrm{H}(50 \mathrm{~A})$ & 7212 & 7136 & 3802 & 22 \\
$\mathrm{H}(50 \mathrm{~B})$ & 6193 & 7030 & 3539 & 22 \\
$\mathrm{H}(51 \mathrm{~A})$ & 6011 & 7545 & 4616 & 33 \\
$\mathrm{H}(51 \mathrm{~B})$ & 7043 & 7518 & 4869 & 33 \\
$\mathrm{H}(52 \mathrm{~A})$ & 6004 & 6052 & 4386 & 37 \\
$\mathrm{H}(52 \mathrm{~B})$ & 6382 & 6241 & 5165 & 37 \\
$\mathrm{H}(53 \mathrm{~A})$ & 7424 & 5846 & 4223 & 65 \\
$\mathrm{H}(53 \mathrm{~B})$ & 7241 & 5205 & 4788 & 65 \\
$\mathrm{H}(53 \mathrm{C})$ & 7809 & 6046 & 5001 & 65 \\
$\mathrm{H}(31)$ & 1739 & 6331 & 3194 & 27 \\
$\mathrm{H}(28)$ & 189 & 9019 & 1778 & 20 \\
& & & & \\
\hline
\end{tabular}


Table 16. Crystal data and structure refinement for $\{\mathrm{ASN}\}_{2}\left\{\left[\mathrm{Ph}_{3} \mathrm{BP}^{m-\mathrm{Ph} 2}\right]_{2} \mathrm{PtMe}_{2}\right\}$.

Empirical formula

Formula weight

Crystal Habit

Crystal Color

Crystal size

Type of diffractometer

Wavelength

Data collection temperature

Unit cell dimensions

Volume

Z

Crystal system

Space group

Density (calculated)

$\mathrm{F}(000)$

$\theta$ range for data collection

Completeness to $\theta=28.59^{\circ}$

Index ranges

Reflections collected

Independent reflections

Absorption coefficient

Absorption correction

Structure solution program

Primary solution method

Secondary solution method

Hydrogen placement

Structure refinement program

Refinement method

Data / restraints / parameters

Goodness-of-fit on $\mathrm{F}^{2}$

Final $\mathrm{R}$ indices $[\mathrm{I}>2 \sigma(\mathrm{I})]$
$\mathrm{C}_{102} \mathrm{H}_{122} \mathrm{~B}_{2} \mathrm{BrN}_{3} \mathrm{OP}_{2} \mathrm{Pt}$

1764.59

plate

colorless

$0.27 \times 0.26 \times 0.13 \mathrm{~mm}^{3}$

\section{Data Collection}

CCD area detector

$0.71073 \AA$

98(2) K

$\mathrm{a}=10.1511(18) \AA$

$\alpha=76.949(3)^{\circ}$

$\mathrm{b}=17.534(3) \AA$

$\beta=85.774(3)^{\circ}$

$\mathrm{c}=24.912(4) \AA$

$\gamma=83.253(3)^{\circ}$

4284.4(13) $\AA^{3}$

2

Triclinic

P-1

$1.368 \mathrm{Mg} / \mathrm{m}^{3}$

1832

1.30 to $28.59^{\circ}$

$91.7 \%$

$-13 \leq \mathrm{h} \leq 13,-23 \leq \mathrm{k} \leq 23,-33 \leq 1 \leq 32$

89517

$20077[\mathrm{R}(\mathrm{int})=0.0702]$

$2.193 \mathrm{~mm}^{-1}$

None

\section{Structure solution and refinement}

SHELXS-97 (Sheldrick, 1990)

direct methods

differential Fourier Map

calculated positions

SHELXL-97 (Sheldrick, 1997)

Full-matrix least-squares on $\mathrm{F}^{2}$

20077 / 0 / 1013

1.103

$\mathrm{R} 1=0.0413, \mathrm{wR} 2=0.0947$ 
$\mathrm{R}$ indices (all data)

Type of weighting scheme used

Weighting scheme used

$\mathrm{P}=\left(\mathrm{Fo}^{\wedge} 2^{\wedge}+2 \mathrm{Fc}^{\wedge} 2^{\wedge}\right) / 3$

Max shift/error

Average shift/error

Largest diff. peak and hole

Special Refinement Details:

The complex co-crystallized with [ASN]Br and one highly disordered molecule of $\mathrm{Et}_{2} \mathrm{O}$.
$\mathrm{R} 1=0.0535, \mathrm{wR} 2=0.0992$

calc calc $\mathrm{w}=1 /\left[{ }^{\wedge} 2^{\wedge}\left(\mathrm{Fo}^{\wedge} 2^{\wedge}\right)+(0.0341 \mathrm{P})^{\wedge} 2^{\wedge}+11.1403 \mathrm{P}\right]$ where

2.395

0.045

2.339 and $-1.763 \mathrm{e} \cdot \AA^{-3}$ 
Table 17. Atomic coordinates (x $\left.10^{4}\right)$ and equivalent isotropic displacement parameters $\left(\AA^{2} \mathrm{x}\right.$ $\left.10^{3}\right)$ for $\left\{\mathrm{ASN}_{2}\left\{\left[\mathrm{Ph}_{3} \mathrm{BP}^{m-\mathrm{Ph} 2}\right]_{2} \mathrm{PtMe}_{2}\right\}\right.$. U(eq) is defined as one third of the trace of the orthogonalized Uij tensor.

\begin{tabular}{|c|c|c|c|c|}
\hline & $\mathrm{x}$ & $\mathrm{y}$ & $\mathrm{z}$ & $\mathrm{U}(\mathrm{eq})$ \\
\hline$\overline{\mathrm{Pt}(1)}$ & $10128(1)$ & $9497(1)$ & $3235(1)$ & $13(1)$ \\
\hline $\mathrm{B}(1)$ & $6405(4)$ & $11748(3)$ & $795(2)$ & $22(1)$ \\
\hline $\mathrm{C}(1)$ & $11053(4)$ & $10408(2)$ & $3431(2)$ & $24(1)$ \\
\hline$C(2)$ & 11622(4) & $8741(2)$ & $3719(2)$ & 19(1) \\
\hline $\mathrm{P}(1)$ & $8598(1)$ & $10426(1)$ & $2768(1)$ & $14(1)$ \\
\hline$C(3)$ & $6407(4)$ & $11635(3)$ & $157(2)$ & $26(1)$ \\
\hline$C(4)$ & $7209(5)$ & $12067(3)$ & $-263(2)$ & $36(1)$ \\
\hline$C(5)$ & $7317(5)$ & 11946(3) & $-798(2)$ & $44(1)$ \\
\hline$C(6)$ & $6609(5)$ & 11397(3) & $-939(2)$ & $36(1)$ \\
\hline$C(7)$ & $5789(5)$ & $10980(3)$ & $-546(2)$ & $33(1)$ \\
\hline$C(8)$ & $5706(4)$ & $11095(2)$ & $-4(2)$ & $28(1)$ \\
\hline $\mathrm{C}(9)$ & 4985(4) & $11535(2)$ & $1137(2)$ & $21(1)$ \\
\hline $\mathrm{C}(10)$ & $4719(4)$ & $10747(2)$ & $1322(2)$ & $25(1)$ \\
\hline $\mathrm{C}(11)$ & $3560(4)$ & $10522(2)$ & $1614(2)$ & $25(1)$ \\
\hline $\mathrm{C}(12)$ & 2597(4) & $11090(3)$ & $1748(2)$ & $25(1)$ \\
\hline $\mathrm{C}(13)$ & 2812(4) & $11869(3)$ & $1579(2)$ & $24(1)$ \\
\hline $\mathrm{C}(14)$ & 3979(4) & $12091(2)$ & $1282(2)$ & 19(1) \\
\hline$C(15)$ & $6696(4)$ & $12659(2)$ & $790(2)$ & $28(1)$ \\
\hline$C(16)$ & $5932(5)$ & $13306(3)$ & 481(3) & $47(2)$ \\
\hline $\mathrm{C}(17)$ & $6189(6)$ & 14093(3) & $465(3)$ & $58(2)$ \\
\hline $\mathrm{C}(18)$ & $7220(7)$ & $14251(3)$ & $730(3)$ & $54(2)$ \\
\hline$C(19)$ & $7990(7)$ & $13636(3)$ & 1031(2) & $47(2)$ \\
\hline $\mathrm{C}(20)$ & 7713(5) & $12860(3)$ & $1060(2)$ & $32(1)$ \\
\hline $\mathrm{C}(21)$ & 7570(4) & $11123(2)$ & $1130(2)$ & $17(1)$ \\
\hline$C(22)$ & $7605(3)$ & $11024(2)$ & $1706(2)$ & $15(1)$ \\
\hline$C(23)$ & $8530(4)$ & $10501(2)$ & $2030(2)$ & $14(1)$ \\
\hline $\mathrm{C}(24)$ & 9513(3) & $10060(2)$ & $1769(2)$ & $15(1)$ \\
\hline$C(25)$ & $9527(4)$ & $10148(2)$ & $1201(2)$ & $18(1)$ \\
\hline$C(26)$ & $8571(4)$ & $10667(2)$ & $890(2)$ & $18(1)$ \\
\hline $\mathrm{C}(27)$ & $8757(4)$ & $11460(2)$ & 2784(2) & $18(1)$ \\
\hline $\mathrm{C}(28)$ & $9405(4)$ & 11941(2) & $2345(2)$ & $21(1)$ \\
\hline$C(29)$ & $9584(4)$ & $12711(2)$ & $2361(2)$ & $25(1)$ \\
\hline $\mathrm{C}(30)$ & $9125(4)$ & $13012(2)$ & $2821(2)$ & $26(1)$ \\
\hline $\mathrm{C}(31)$ & $8495(4)$ & $12537(2)$ & $3260(2)$ & $25(1)$ \\
\hline $\mathrm{C}(32)$ & $8316(4)$ & $11768(2)$ & $3243(2)$ & $21(1)$ \\
\hline $\mathrm{C}(33)$ & $6946(3)$ & $10294(2)$ & $3098(2)$ & $15(1)$ \\
\hline$C(34)$ & $5764(4)$ & $10343(2)$ & $2832(2)$ & $18(1)$ \\
\hline$C(35)$ & $4577(4)$ & $10220(2)$ & $3142(2)$ & $21(1)$ \\
\hline$C(36)$ & $4551(4)$ & $10063(2)$ & $3712(2)$ & $23(1)$ \\
\hline $\mathrm{C}(37)$ & $5719(4)$ & $10017(2)$ & $3982(2)$ & $23(1)$ \\
\hline $\mathrm{C}(38)$ & $6908(4)$ & $10120(2)$ & $3677(2)$ & 19(1) \\
\hline $\mathrm{B}(2)$ & $9769(4)$ & $5835(2)$ & 2191(2) & $16(1)$ \\
\hline $\mathrm{P}(2)$ & $9247(1)$ & $8376(1)$ & $3151(1)$ & $12(1)$ \\
\hline $\mathrm{C}(39)$ & $8274(4)$ & $6046(2)$ & $1937(2)$ & $18(1)$ \\
\hline $\mathrm{C}(40)$ & $7556(4)$ & $6796(2)$ & $1815(2)$ & $18(1)$ \\
\hline $\mathrm{C}(41)$ & $6268(4)$ & $6924(2)$ & 1621(2) & $23(1)$ \\
\hline
\end{tabular}




\begin{tabular}{|c|c|c|c|c|}
\hline$C(42)$ & $5652(4)$ & $6305(3)$ & 1531(2) & $26(1)$ \\
\hline$C(43)$ & $6344(4)$ & $5558(3)$ & $1636(2)$ & $26(1)$ \\
\hline$C(44)$ & 7618(4) & $5431(2)$ & $1833(2)$ & $21(1)$ \\
\hline C(45) & $10736(4)$ & $5424(2)$ & 1751(2) & $17(1)$ \\
\hline $\mathrm{C}(46)$ & $11176(4)$ & $5884(2)$ & $1246(2)$ & $23(1)$ \\
\hline$C(47)$ & $11994(5)$ & $5574(3)$ & $860(2)$ & $28(1)$ \\
\hline C(48) & $12412(5)$ & $4767(3)$ & $962(2)$ & $31(1)$ \\
\hline C(49) & $11973(5)$ & 4293(3) & $1446(2)$ & $30(1)$ \\
\hline $\mathrm{C}(50)$ & $11157(4)$ & $4616(2)$ & $1833(2)$ & $23(1)$ \\
\hline $\mathrm{C}(51)$ & $9692(4)$ & $5228(2)$ & 2803(2) & $16(1)$ \\
\hline$C(52)$ & $10870(4)$ & 4897(2) & $3067(2)$ & $20(1)$ \\
\hline$C(53)$ & $10876(4)$ & $4377(2)$ & $3578(2)$ & $23(1)$ \\
\hline $\mathrm{C}(54)$ & $9677(4)$ & $4176(2)$ & $3855(2)$ & $23(1)$ \\
\hline$C(55)$ & $8500(4)$ & $4496(2)$ & $3613(2)$ & $24(1)$ \\
\hline$C(56)$ & $8514(4)$ & $5016(2)$ & 3098(2) & $20(1)$ \\
\hline $\mathrm{C}(57)$ & $10424(4)$ & $6602(2)$ & $2302(2)$ & $15(1)$ \\
\hline $\mathrm{C}(58)$ & $9690(3)$ & $7124(2)$ & $2596(2)$ & $14(1)$ \\
\hline C(59) & $10223(4)$ & $7745(2)$ & 2744(1) & $13(1)$ \\
\hline $\mathrm{C}(60)$ & 11549(4) & $7870(2)$ & 2589(2) & $15(1)$ \\
\hline $\mathrm{C}(61)$ & $12313(4)$ & $7360(2)$ & $2307(2)$ & $17(1)$ \\
\hline $\mathrm{C}(62)$ & $11759(4)$ & $6746(2)$ & $2170(2)$ & $17(1)$ \\
\hline$C(63)$ & $9005(4)$ & $7722(2)$ & $3837(2)$ & $18(1)$ \\
\hline$C(64)$ & 8989(4) & $6909(3)$ & 3917(2) & $26(1)$ \\
\hline $\mathrm{C}(65)$ & $8661(5)$ & $6466(3)$ & $4437(2)$ & $35(1)$ \\
\hline$C(66)$ & $8357(5)$ & $6825(3)$ & $4880(2)$ & $40(1)$ \\
\hline $\mathrm{C}(67)$ & $8417(5)$ & $7620(3)$ & $4807(2)$ & $39(1)$ \\
\hline$C(68)$ & $8740(4)$ & $8068(3)$ & $4287(2)$ & $28(1)$ \\
\hline $\mathrm{C}(69)$ & $7587(4)$ & $8443(2)$ & $2883(2)$ & $14(1)$ \\
\hline$C(70)$ & $6458(4)$ & $8311(2)$ & $3232(2)$ & $17(1)$ \\
\hline$C(71)$ & $5214(4)$ & $8386(2)$ & $3017(2)$ & $21(1)$ \\
\hline$C(72)$ & 5077(4) & $8594(2)$ & $2454(2)$ & $21(1)$ \\
\hline$C(73)$ & $6195(4)$ & $8737(2)$ & 2096(2) & $18(1)$ \\
\hline$C(74)$ & 7439(4) & $8660(2)$ & $2312(2)$ & $15(1)$ \\
\hline $\mathrm{N}(1)$ & $1621(4)$ & $2218(2)$ & 9990(1) & $24(1)$ \\
\hline$C(75)$ & $764(5)$ & 2718(3) & $10318(2)$ & $42(1)$ \\
\hline$C(76)$ & $956(6)$ & $3565(3)$ & $10040(2)$ & $45(1)$ \\
\hline$C(77)$ & $2344(6)$ & $3514(3)$ & $9748(2)$ & $38(1)$ \\
\hline$C(78)$ & 2862(4) & $2644(2)$ & 9871(2) & $26(1)$ \\
\hline$C(79)$ & $1025(5)$ & 2181(3) & $9459(2)$ & $28(1)$ \\
\hline $\mathrm{C}(80)$ & $1768(5)$ & $1451(3)$ & $9312(2)$ & $32(1)$ \\
\hline $\mathrm{C}(81)$ & $1858(5)$ & $869(3)$ & $9868(2)$ & $38(1)$ \\
\hline $\mathrm{C}(82)$ & $1896(5)$ & $1360(3)$ & $10297(2)$ & $31(1)$ \\
\hline $\mathrm{N}(2)$ & $3507(3)$ & $5920(2)$ & $4021(2)$ & $24(1)$ \\
\hline$C(83)$ & 4001(4) & $3560(2)$ & 2959(2) & $24(1)$ \\
\hline $\mathrm{C}(84)$ & $5217(5)$ & 3915(3) & 2664(2) & $37(1)$ \\
\hline $\mathrm{C}(85)$ & $5494(6)$ & $3482(3)$ & 2193(2) & $49(1)$ \\
\hline$C(86)$ & $5261(5)$ & $2645(3)$ & $2457(2)$ & $30(1)$ \\
\hline C(87) & $4785(4)$ & $2212(2)$ & $3487(2)$ & $24(1)$ \\
\hline C(88) & $3547(5)$ & 2102(3) & $3876(2)$ & $30(1)$ \\
\hline C(89) & 2361(5) & $2258(3)$ & $3499(2)$ & $36(1)$ \\
\hline $\mathrm{C}(90)$ & 2987(5) & 2341(3) & $2919(2)$ & $33(1)$ \\
\hline $\mathrm{N}(3)$ & $4264(3)$ & $2688(2)$ & 2948(1) & $22(1)$ \\
\hline$C(91)$ & $4887(4)$ & $5831(2)$ & $4246(2)$ & $25(1)$ \\
\hline $\mathrm{C}(92)$ & 5755(4) & $6202(2)$ & $3751(2)$ & $25(1)$ \\
\hline$C(93)$ & $4985(4)$ & $6216(3)$ & $3236(2)$ & $26(1)$ \\
\hline$C(94)$ & 3794(4) & $5770(3)$ & $3454(2)$ & $26(1)$ \\
\hline
\end{tabular}




$\begin{array}{lcccc}\mathrm{C}(95) & 2797(4) & 6728(2) & 4023(2) & 28(1) \\ \mathrm{C}(96) & 2201(4) & 6664(3) & 4613(2) & 27(1) \\ \mathrm{C}(97) & 2134(5) & 5770(3) & 4845(2) & 30(1) \\ \mathrm{C}(98) & 2570(4) & 5385(3) & 4372(2) & 27(1) \\ \mathrm{Br}(1) & 5129(1) & 3704(1) & 4293(1) & 24(1) \\ \mathrm{C}(102) & 4678(9) & 8179(5) & 4712(4) & 87(3) \\ \mathrm{C}(101) & 3609(13) & 8487(5) & 5058(4) & 93(4) \\ \mathrm{C}(100) & 1190(40) & 10366(8) & 4943(7) & 560(50) \\ \mathrm{O}(1) & 2969(14) & 8974(14) & 5167(9) & 406(16) \\ \mathrm{C}(99) & 1710(30) & 9540(30) & 5086(9) & 910(60)\end{array}$


Table 18. Bond lengths $[\AA]$ and angles $\left[{ }^{\circ}\right]$ for $\left\{\mathrm{ASN}_{2}\left\{\left[\mathrm{Ph}_{3} \mathrm{BP}^{m-\mathrm{Ph} 2}\right]_{2} \mathrm{PtMe}_{2}\right\}\right.$.

\begin{tabular}{|c|c|c|c|}
\hline $\mathrm{Pt}(1)-\mathrm{C}(1)$ & $2.107(4)$ & $\mathrm{C}(22)-\mathrm{H}(22)$ & 0.9500 \\
\hline $\operatorname{Pt}(1)-C(2)$ & $2.141(4)$ & $C(23)-C(24)$ & $1.402(5)$ \\
\hline $\mathrm{Pt}(1)-\mathrm{P}(1)$ & $2.2885(10)$ & $\mathrm{C}(24)-\mathrm{C}(25)$ & $1.387(5)$ \\
\hline $\operatorname{Pt}(1)-\mathrm{P}(2)$ & $2.3096(10)$ & $\mathrm{C}(24)-\mathrm{H}(24)$ & 0.9500 \\
\hline $\mathrm{B}(1)-\mathrm{C}(21)$ & $1.651(6)$ & $C(25)-C(26)$ & $1.399(5)$ \\
\hline $\mathrm{B}(1)-\mathrm{C}(9)$ & $1.656(6)$ & $\mathrm{C}(25)-\mathrm{H}(25)$ & 0.9500 \\
\hline $\mathrm{B}(1)-\mathrm{C}(3)$ & $1.645(7)$ & $\mathrm{C}(26)-\mathrm{H}(26)$ & 0.9500 \\
\hline $\mathrm{B}(1)-\mathrm{C}(15)$ & $1.655(6)$ & $\mathrm{C}(27)-\mathrm{C}(28)$ & $1.394(6)$ \\
\hline $\mathrm{C}(1)-\mathrm{H}(1 \mathrm{~A})$ & 0.9800 & $C(27)-C(32)$ & $1.395(6)$ \\
\hline $\mathrm{C}(1)-\mathrm{H}(1 \mathrm{~B})$ & 0.9800 & $C(28)-C(29)$ & $1.393(5)$ \\
\hline $\mathrm{C}(1)-\mathrm{H}(1 \mathrm{C})$ & 0.9800 & $\mathrm{C}(28)-\mathrm{H}(28)$ & 0.9500 \\
\hline $\mathrm{C}(2)-\mathrm{H}(2 \mathrm{~A})$ & 0.9800 & $C(29)-C(30)$ & $1.396(6)$ \\
\hline $\mathrm{C}(2)-\mathrm{H}(2 \mathrm{~B})$ & 0.9800 & $\mathrm{C}(29)-\mathrm{H}(29)$ & 0.9500 \\
\hline $\mathrm{C}(2)-\mathrm{H}(2 \mathrm{C})$ & 0.9800 & $\mathrm{C}(30)-\mathrm{C}(31)$ & $1.381(6)$ \\
\hline $\mathrm{P}(1)-\mathrm{C}(23)$ & $1.819(4)$ & $\mathrm{C}(30)-\mathrm{H}(30)$ & 0.9500 \\
\hline $\mathrm{P}(1)-\mathrm{C}(33)$ & $1.828(4)$ & $\mathrm{C}(31)-\mathrm{C}(32)$ & $1.393(5)$ \\
\hline $\mathrm{P}(1)-\mathrm{C}(27)$ & $1.848(4)$ & $\mathrm{C}(31)-\mathrm{H}(31)$ & 0.9500 \\
\hline $\mathrm{C}(3)-\mathrm{C}(8)$ & $1.392(6)$ & $\mathrm{C}(32)-\mathrm{H}(32)$ & 0.9500 \\
\hline $\mathrm{C}(3)-\mathrm{C}(4)$ & $1.411(6)$ & $\mathrm{C}(33)-\mathrm{C}(34)$ & $1.397(5)$ \\
\hline$C(4)-C(5)$ & $1.391(7)$ & $\mathrm{C}(33)-\mathrm{C}(38)$ & $1.403(5)$ \\
\hline $\mathrm{C}(4)-\mathrm{H}(4)$ & 0.9500 & $C(34)-C(35)$ & $1.395(5)$ \\
\hline$C(5)-C(6)$ & $1.385(7)$ & $\mathrm{C}(34)-\mathrm{H}(34)$ & 0.9500 \\
\hline $\mathrm{C}(5)-\mathrm{H}(5)$ & 0.9500 & $C(35)-C(36)$ & $1.382(6)$ \\
\hline$C(6)-C(7)$ & $1.375(7)$ & $\mathrm{C}(35)-\mathrm{H}(35)$ & 0.9500 \\
\hline $\mathrm{C}(6)-\mathrm{H}(6)$ & 0.9500 & $C(36)-C(37)$ & $1.391(6)$ \\
\hline $\mathrm{C}(7)-\mathrm{C}(8)$ & $1.404(7)$ & $\mathrm{C}(36)-\mathrm{H}(36)$ & 0.9500 \\
\hline $\mathrm{C}(7)-\mathrm{H}(7)$ & 0.9500 & $\mathrm{C}(37)-\mathrm{C}(38)$ & $1.387(5)$ \\
\hline $\mathrm{C}(8)-\mathrm{H}(8)$ & 0.9500 & $\mathrm{C}(37)-\mathrm{H}(37)$ & 0.9500 \\
\hline$C(9)-C(14)$ & $1.413(6)$ & $\mathrm{C}(38)-\mathrm{H}(38)$ & 0.9500 \\
\hline$C(9)-C(10)$ & $1.407(6)$ & $\mathrm{B}(2)-\mathrm{C}(45)$ & $1.650(6)$ \\
\hline $\mathrm{C}(10)-\mathrm{C}(11)$ & $1.386(6)$ & $\mathrm{B}(2)-\mathrm{C}(39)$ & $1.656(6)$ \\
\hline $\mathrm{C}(10)-\mathrm{H}(10)$ & 0.9500 & $\mathrm{~B}(2)-\mathrm{C}(51)$ & $1.653(6)$ \\
\hline $\mathrm{C}(11)-\mathrm{C}(12)$ & $1.391(6)$ & $\mathrm{B}(2)-\mathrm{C}(57)$ & $1.653(5)$ \\
\hline $\mathrm{C}(11)-\mathrm{H}(11)$ & 0.9500 & $\mathrm{P}(2)-\mathrm{C}(59)$ & $1.825(4)$ \\
\hline$C(12)-C(13)$ & $1.373(6)$ & $\mathrm{P}(2)-\mathrm{C}(69)$ & $1.840(4)$ \\
\hline $\mathrm{C}(12)-\mathrm{H}(12)$ & 0.9500 & $P(2)-C(63)$ & $1.848(4)$ \\
\hline $\mathrm{C}(13)-\mathrm{C}(14)$ & $1.396(6)$ & $\mathrm{C}(39)-\mathrm{C}(40)$ & $1.407(5)$ \\
\hline $\mathrm{C}(13)-\mathrm{H}(13)$ & 0.9500 & $C(39)-C(44)$ & $1.413(5)$ \\
\hline $\mathrm{C}(14)-\mathrm{H}(14)$ & 0.9500 & $\mathrm{C}(40)-\mathrm{C}(41)$ & $1.403(5)$ \\
\hline$C(15)-C(20)$ & $1.393(7)$ & $\mathrm{C}(40)-\mathrm{H}(40)$ & 0.9500 \\
\hline$C(15)-C(16)$ & $1.403(6)$ & $\mathrm{C}(41)-\mathrm{C}(42)$ & $1.383(6)$ \\
\hline$C(16)-C(17)$ & $1.425(8)$ & $\mathrm{C}(41)-\mathrm{H}(41)$ & 0.9500 \\
\hline $\mathrm{C}(16)-\mathrm{H}(16)$ & 0.9500 & $C(42)-C(43)$ & $1.390(6)$ \\
\hline $\mathrm{C}(17)-\mathrm{C}(18)$ & $1.363(10)$ & $\mathrm{C}(42)-\mathrm{H}(42)$ & 0.9500 \\
\hline $\mathrm{C}(17)-\mathrm{H}(17)$ & 0.9500 & $\mathrm{C}(43)-\mathrm{C}(44)$ & $1.392(6)$ \\
\hline $\mathrm{C}(18)-\mathrm{C}(19)$ & $1.364(9)$ & $\mathrm{C}(43)-\mathrm{H}(43)$ & 0.9500 \\
\hline $\mathrm{C}(18)-\mathrm{H}(18)$ & 0.9500 & $\mathrm{C}(44)-\mathrm{H}(44)$ & 0.9500 \\
\hline$C(19)-C(20)$ & $1.406(6)$ & $\mathrm{C}(45)-\mathrm{C}(50)$ & $1.404(5)$ \\
\hline $\mathrm{C}(19)-\mathrm{H}(19)$ & 0.9500 & $\mathrm{C}(45)-\mathrm{C}(46)$ & $1.404(6)$ \\
\hline $\mathrm{C}(20)-\mathrm{H}(20)$ & 0.9500 & $\mathrm{C}(46)-\mathrm{C}(47)$ & $1.387(6)$ \\
\hline$C(21)-C(26)$ & $1.402(5)$ & $\mathrm{C}(46)-\mathrm{H}(46)$ & 0.9500 \\
\hline$C(21)-C(22)$ & $1.407(5)$ & $\mathrm{C}(47)-\mathrm{C}(48)$ & $1.399(6)$ \\
\hline $\mathrm{C}(22)-\mathrm{C}(23)$ & $1.397(5)$ & $\mathrm{C}(47)-\mathrm{H}(47)$ & 0.9500 \\
\hline
\end{tabular}




\begin{tabular}{|c|c|c|c|}
\hline $\mathrm{C}(48)-\mathrm{C}(49)$ & $1.378(6)$ & $\mathrm{C}(76)-\mathrm{H}(76 \mathrm{~A})$ & 0.9900 \\
\hline $\mathrm{C}(48)-\mathrm{H}(48)$ & 0.9500 & $\mathrm{C}(76)-\mathrm{H}(76 \mathrm{~B})$ & 0.9900 \\
\hline$C(49)-C(50)$ & $1.397(6)$ & $C(77)-C(78)$ & $1.524(6)$ \\
\hline $\mathrm{C}(49)-\mathrm{H}(49)$ & 0.9500 & $\mathrm{C}(77)-\mathrm{H}(77 \mathrm{~A})$ & 0.9900 \\
\hline $\mathrm{C}(50)-\mathrm{H}(50)$ & 0.9500 & $\mathrm{C}(77)-\mathrm{H}(77 \mathrm{~B})$ & 0.9900 \\
\hline$C(51)-C(56)$ & $1.400(5)$ & $\mathrm{C}(78)-\mathrm{H}(78 \mathrm{~A})$ & 0.9900 \\
\hline $\mathrm{C}(51)-\mathrm{C}(52)$ & $1.408(5)$ & $\mathrm{C}(78)-\mathrm{H}(78 \mathrm{~B})$ & 0.9900 \\
\hline $\mathrm{C}(52)-\mathrm{C}(53)$ & $1.388(6)$ & $\mathrm{C}(79)-\mathrm{C}(80)$ & $1.511(6)$ \\
\hline $\mathrm{C}(52)-\mathrm{H}(52)$ & 0.9500 & $\mathrm{C}(79)-\mathrm{H}(79 \mathrm{~A})$ & 0.9900 \\
\hline$C(53)-C(54)$ & $1.396(6)$ & $\mathrm{C}(79)-\mathrm{H}(79 \mathrm{~B})$ & 0.9900 \\
\hline $\mathrm{C}(53)-\mathrm{H}(53)$ & 0.9500 & $\mathrm{C}(80)-\mathrm{C}(81)$ & $1.526(7)$ \\
\hline$C(54)-C(55)$ & $1.379(6)$ & $\mathrm{C}(80)-\mathrm{H}(80 \mathrm{~A})$ & 0.9900 \\
\hline $\mathrm{C}(54)-\mathrm{H}(54)$ & 0.9500 & $\mathrm{C}(80)-\mathrm{H}(80 \mathrm{~B})$ & 0.9900 \\
\hline$C(55)-C(56)$ & $1.397(6)$ & $\mathrm{C}(81)-\mathrm{C}(82)$ & $1.522(7)$ \\
\hline $\mathrm{C}(55)-\mathrm{H}(55)$ & 0.9500 & $\mathrm{C}(81)-\mathrm{H}(81 \mathrm{~A})$ & 0.9900 \\
\hline $\mathrm{C}(56)-\mathrm{H}(56)$ & 0.9500 & $\mathrm{C}(81)-\mathrm{H}(81 \mathrm{~B})$ & 0.9900 \\
\hline$C(57)-C(58)$ & $1.411(5)$ & $\mathrm{C}(82)-\mathrm{H}(82 \mathrm{~A})$ & 0.9900 \\
\hline$C(57)-C(62)$ & $1.409(5)$ & $\mathrm{C}(82)-\mathrm{H}(82 \mathrm{~B})$ & 0.9900 \\
\hline $\mathrm{C}(58)-\mathrm{C}(59)$ & $1.402(5)$ & $\mathrm{N}(2)-\mathrm{C}(94)$ & $1.496(6)$ \\
\hline $\mathrm{C}(58)-\mathrm{H}(58)$ & 0.9500 & $\mathrm{~N}(2)-\mathrm{C}(98)$ & $1.505(5)$ \\
\hline$C(59)-C(60)$ & $1.402(5)$ & $\mathrm{N}(2)-\mathrm{C}(95)$ & $1.513(5)$ \\
\hline$C(60)-C(61)$ & $1.390(5)$ & $\mathrm{N}(2)-\mathrm{C}(91)$ & $1.525(5)$ \\
\hline $\mathrm{C}(60)-\mathrm{H}(60)$ & 0.9500 & $\mathrm{C}(83)-\mathrm{C}(84)$ & $1.513(6)$ \\
\hline$C(61)-C(62)$ & $1.389(5)$ & $\mathrm{C}(83)-\mathrm{N}(3)$ & $1.526(5)$ \\
\hline $\mathrm{C}(61)-\mathrm{H}(61)$ & 0.9500 & $\mathrm{C}(83)-\mathrm{H}(83 \mathrm{~A})$ & 0.9900 \\
\hline $\mathrm{C}(62)-\mathrm{H}(62)$ & 0.9500 & $\mathrm{C}(83)-\mathrm{H}(83 \mathrm{~B})$ & 0.9900 \\
\hline $\mathrm{C}(63)-\mathrm{C}(68)$ & $1.387(6)$ & $\mathrm{C}(84)-\mathrm{C}(85)$ & $1.529(7)$ \\
\hline$C(63)-C(64)$ & $1.395(6)$ & $\mathrm{C}(84)-\mathrm{H}(84 \mathrm{~A})$ & 0.9900 \\
\hline$C(64)-C(65)$ & $1.391(6)$ & $\mathrm{C}(84)-\mathrm{H}(84 \mathrm{~B})$ & 0.9900 \\
\hline $\mathrm{C}(64)-\mathrm{H}(64)$ & 0.9500 & $\mathrm{C}(85)-\mathrm{C}(86)$ & $1.505(7)$ \\
\hline$C(65)-C(66)$ & $1.388(8)$ & $\mathrm{C}(85)-\mathrm{H}(85 \mathrm{~A})$ & 0.9900 \\
\hline $\mathrm{C}(65)-\mathrm{H}(65)$ & 0.9500 & $\mathrm{C}(85)-\mathrm{H}(85 \mathrm{~B})$ & 0.9900 \\
\hline$C(66)-C(67)$ & $1.372(8)$ & $\mathrm{C}(86)-\mathrm{N}(3)$ & $1.537(5)$ \\
\hline $\mathrm{C}(66)-\mathrm{H}(66)$ & 0.9500 & $\mathrm{C}(86)-\mathrm{H}(86 \mathrm{~A})$ & 0.9900 \\
\hline $\mathrm{C}(67)-\mathrm{C}(68)$ & $1.393(6)$ & $\mathrm{C}(86)-\mathrm{H}(86 \mathrm{~B})$ & 0.9900 \\
\hline $\mathrm{C}(67)-\mathrm{H}(67)$ & 0.9500 & C(87)-N(3) & $1.508(5)$ \\
\hline $\mathrm{C}(68)-\mathrm{H}(68)$ & 0.9500 & $\mathrm{C}(87)-\mathrm{C}(88)$ & $1.532(6)$ \\
\hline $\mathrm{C}(69)-\mathrm{C}(70)$ & $1.396(5)$ & $\mathrm{C}(87)-\mathrm{H}(87 \mathrm{~A})$ & 0.9900 \\
\hline$C(69)-C(74)$ & $1.400(5)$ & $\mathrm{C}(87)-\mathrm{H}(87 \mathrm{~B})$ & 0.9900 \\
\hline$C(70)-C(71)$ & $1.390(5)$ & $\mathrm{C}(88)-\mathrm{C}(89)$ & $1.543(7)$ \\
\hline $\mathrm{C}(70)-\mathrm{H}(70)$ & 0.9500 & $\mathrm{C}(88)-\mathrm{H}(88 \mathrm{~A})$ & 0.9900 \\
\hline$C(71)-C(72)$ & $1.380(6)$ & $\mathrm{C}(88)-\mathrm{H}(88 \mathrm{~B})$ & 0.9900 \\
\hline $\mathrm{C}(71)-\mathrm{H}(71)$ & 0.9500 & C(89)-C(90) & $1.518(7)$ \\
\hline$C(72)-C(73)$ & $1.401(6)$ & $\mathrm{C}(89)-\mathrm{H}(89 \mathrm{~A})$ & 0.9900 \\
\hline $\mathrm{C}(72)-\mathrm{H}(72)$ & 0.9500 & $\mathrm{C}(89)-\mathrm{H}(89 \mathrm{~B})$ & 0.9900 \\
\hline$C(73)-C(74)$ & $1.388(5)$ & $\mathrm{C}(90)-\mathrm{N}(3)$ & $1.509(5)$ \\
\hline $\mathrm{C}(73)-\mathrm{H}(73)$ & 0.9500 & $\mathrm{C}(90)-\mathrm{H}(90 \mathrm{~A})$ & 0.9900 \\
\hline $\mathrm{C}(74)-\mathrm{H}(74)$ & 0.9500 & $\mathrm{C}(90)-\mathrm{H}(90 \mathrm{~B})$ & 0.9900 \\
\hline $\mathrm{N}(1)-\mathrm{C}(75)$ & $1.493(6)$ & $\mathrm{C}(91)-\mathrm{C}(92)$ & $1.527(6)$ \\
\hline $\mathrm{N}(1)-\mathrm{C}(78)$ & $1.515(6)$ & $\mathrm{C}(91)-\mathrm{H}(91 \mathrm{~A})$ & 0.9900 \\
\hline $\mathrm{N}(1)-\mathrm{C}(79)$ & $1.514(5)$ & $\mathrm{C}(91)-\mathrm{H}(91 \mathrm{~B})$ & 0.9900 \\
\hline $\mathrm{N}(1)-\mathrm{C}(82)$ & $1.529(5)$ & $\mathrm{C}(92)-\mathrm{C}(93)$ & $1.544(6)$ \\
\hline$C(75)-C(76)$ & $1.519(8)$ & $\mathrm{C}(92)-\mathrm{H}(92 \mathrm{~A})$ & 0.9900 \\
\hline $\mathrm{C}(75)-\mathrm{H}(75 \mathrm{~A})$ & 0.9900 & $\mathrm{C}(92)-\mathrm{H}(92 \mathrm{~B})$ & 0.9900 \\
\hline $\mathrm{C}(75)-\mathrm{H}(75 \mathrm{~B})$ & 0.9900 & $\mathrm{C}(93)-\mathrm{C}(94)$ & $1.516(6)$ \\
\hline$C(76)-C(77)$ & $1.540(8)$ & $\mathrm{C}(93)-\mathrm{H}(93 \mathrm{~A})$ & 0.9900 \\
\hline
\end{tabular}




\begin{tabular}{|c|c|c|c|}
\hline C(93)-H(93B) & 0.9900 & $\mathrm{C}(23)-\mathrm{P}(1)-\mathrm{Pt}(1)$ & $117.59(12)$ \\
\hline $\mathrm{C}(94)-\mathrm{H}(94 \mathrm{~A})$ & 0.9900 & C(33)-P(1)-Pt(1) & $109.60(13)$ \\
\hline C(94)-H(94B) & 0.9900 & C(27)-P(1)-Pt(1) & $116.34(12)$ \\
\hline $\mathrm{C}(95)-\mathrm{C}(96)$ & $1.534(6)$ & $\mathrm{C}(8)-\mathrm{C}(3)-\mathrm{C}(4)$ & $115.4(4)$ \\
\hline $\mathrm{C}(95)-\mathrm{H}(95 \mathrm{~A})$ & 0.9900 & $\mathrm{C}(8)-\mathrm{C}(3)-\mathrm{B}(1)$ & $124.0(4)$ \\
\hline $\mathrm{C}(95)-\mathrm{H}(95 \mathrm{~B})$ & 0.9900 & $\mathrm{C}(4)-\mathrm{C}(3)-\mathrm{B}(1)$ & $120.4(4)$ \\
\hline $\mathrm{C}(96)-\mathrm{C}(97)$ & $1.550(6)$ & $C(5)-C(4)-C(3)$ & $122.2(5)$ \\
\hline $\mathrm{C}(96)-\mathrm{H}(96 \mathrm{~A})$ & 0.9900 & $\mathrm{C}(5)-\mathrm{C}(4)-\mathrm{H}(4)$ & 118.9 \\
\hline $\mathrm{C}(96)-\mathrm{H}(96 \mathrm{~B})$ & 0.9900 & $\mathrm{C}(3)-\mathrm{C}(4)-\mathrm{H}(4)$ & 118.9 \\
\hline $\mathrm{C}(97)-\mathrm{C}(98)$ & $1.501(6)$ & $C(6)-C(5)-C(4)$ & $120.4(4)$ \\
\hline C(97)-H(97A) & 0.9900 & $\mathrm{C}(6)-\mathrm{C}(5)-\mathrm{H}(5)$ & 119.8 \\
\hline C(97)-H(97B) & 0.9900 & $\mathrm{C}(4)-\mathrm{C}(5)-\mathrm{H}(5)$ & 119.8 \\
\hline $\mathrm{C}(98)-\mathrm{H}(98 \mathrm{~A})$ & 0.9900 & $\mathrm{C}(5)-\mathrm{C}(6)-\mathrm{C}(7)$ & $119.2(5)$ \\
\hline C(98)-H(98B) & 0.9900 & $\mathrm{C}(5)-\mathrm{C}(6)-\mathrm{H}(6)$ & 120.4 \\
\hline$C(102)-C(101)$ & $1.466(13)$ & $\mathrm{C}(7)-\mathrm{C}(6)-\mathrm{H}(6)$ & 120.4 \\
\hline $\mathrm{C}(102)-\mathrm{H}(10 \mathrm{~A})$ & 0.9800 & $\mathrm{C}(6)-\mathrm{C}(7)-\mathrm{C}(8)$ & $120.0(5)$ \\
\hline $\mathrm{C}(102)-\mathrm{H}(10 \mathrm{~B})$ & 0.9800 & $\mathrm{C}(6)-\mathrm{C}(7)-\mathrm{H}(7)$ & 120.0 \\
\hline $\mathrm{C}(102)-\mathrm{H}(10 \mathrm{C})$ & 0.9800 & $\mathrm{C}(8)-\mathrm{C}(7)-\mathrm{H}(7)$ & 120.0 \\
\hline $\mathrm{C}(101)-\mathrm{O}(1)$ & $1.082(15)$ & $\mathrm{C}(3)-\mathrm{C}(8)-\mathrm{C}(7)$ & $122.7(4)$ \\
\hline C(101)-H(10D) & 0.9900 & $\mathrm{C}(3)-\mathrm{C}(8)-\mathrm{H}(8)$ & 118.6 \\
\hline $\mathrm{C}(101)-\mathrm{H}(10 \mathrm{E})$ & 0.9900 & $\mathrm{C}(7)-\mathrm{C}(8)-\mathrm{H}(8)$ & 118.6 \\
\hline$C(100)-C(99)$ & $1.45(4)$ & $C(14)-C(9)-C(10)$ & $114.4(4)$ \\
\hline $\mathrm{C}(100)-\mathrm{H}(10 \mathrm{~F})$ & 0.9911 & $\mathrm{C}(14)-\mathrm{C}(9)-\mathrm{B}(1)$ & $125.3(3)$ \\
\hline $\mathrm{C}(100)-\mathrm{H}(10 \mathrm{G})$ & 0.9929 & $\mathrm{C}(10)-\mathrm{C}(9)-\mathrm{B}(1)$ & $120.2(4)$ \\
\hline $\mathrm{C}(100)-\mathrm{H}(10 \mathrm{H})$ & 0.9930 & $\mathrm{C}(11)-\mathrm{C}(10)-\mathrm{C}(9)$ & $123.6(4)$ \\
\hline $\mathrm{O}(1)-\mathrm{C}(99)$ & $1.52(3)$ & $\mathrm{C}(11)-\mathrm{C}(10)-\mathrm{H}(10)$ & 118.2 \\
\hline C(99)-H(99A) & 0.9900 & $\mathrm{C}(9)-\mathrm{C}(10)-\mathrm{H}(10)$ & 118.2 \\
\hline \multirow[t]{2}{*}{ C(99)-H(99B) } & 0.9900 & $\mathrm{C}(10)-\mathrm{C}(11)-\mathrm{C}(12)$ & $119.9(4)$ \\
\hline & & $\mathrm{C}(10)-\mathrm{C}(11)-\mathrm{H}(11)$ & 120.0 \\
\hline$C(1)-P t(1)-C(2)$ & $84.19(15)$ & $\mathrm{C}(12)-\mathrm{C}(11)-\mathrm{H}(11)$ & 120.0 \\
\hline $\mathrm{C}(1)-\mathrm{Pt}(1)-\mathrm{P}(1)$ & $88.94(11)$ & $\mathrm{C}(13)-\mathrm{C}(12)-\mathrm{C}(11)$ & $118.7(4)$ \\
\hline $\mathrm{C}(2)-\mathrm{Pt}(1)-\mathrm{P}(1)$ & $172.86(10)$ & $\mathrm{C}(13)-\mathrm{C}(12)-\mathrm{H}(12)$ & 120.6 \\
\hline $\mathrm{C}(1)-\mathrm{Pt}(1)-\mathrm{P}(2)$ & $170.80(12)$ & $\mathrm{C}(11)-\mathrm{C}(12)-\mathrm{H}(12)$ & 120.6 \\
\hline $\mathrm{C}(2)-\mathrm{Pt}(1)-\mathrm{P}(2)$ & $87.54(10)$ & $C(12)-C(13)-C(14)$ & $121.0(4)$ \\
\hline $\mathrm{P}(1)-\mathrm{Pt}(1)-\mathrm{P}(2)$ & $99.17(3)$ & $\mathrm{C}(12)-\mathrm{C}(13)-\mathrm{H}(13)$ & 119.5 \\
\hline $\mathrm{C}(21)-\mathrm{B}(1)-\mathrm{C}(9)$ & $105.8(3)$ & $\mathrm{C}(14)-\mathrm{C}(13)-\mathrm{H}(13)$ & 119.5 \\
\hline $\mathrm{C}(21)-\mathrm{B}(1)-\mathrm{C}(3)$ & $109.8(3)$ & $C(13)-C(14)-C(9)$ & $122.3(4)$ \\
\hline $\mathrm{C}(9)-\mathrm{B}(1)-\mathrm{C}(3)$ & $111.2(3)$ & $\mathrm{C}(13)-\mathrm{C}(14)-\mathrm{H}(14)$ & 118.9 \\
\hline $\mathrm{C}(21)-\mathrm{B}(1)-\mathrm{C}(15)$ & $109.4(3)$ & $\mathrm{C}(9)-\mathrm{C}(14)-\mathrm{H}(14)$ & 118.9 \\
\hline $\mathrm{C}(9)-\mathrm{B}(1)-\mathrm{C}(15)$ & 111.1(3) & $\mathrm{C}(20)-\mathrm{C}(15)-\mathrm{C}(16)$ & $114.1(4)$ \\
\hline $\mathrm{C}(3)-\mathrm{B}(1)-\mathrm{C}(15)$ & $109.5(3)$ & $\mathrm{C}(20)-\mathrm{C}(15)-\mathrm{B}(1)$ & $125.0(4)$ \\
\hline $\mathrm{Pt}(1)-\mathrm{C}(1)-\mathrm{H}(1 \mathrm{~A})$ & 109.5 & $\mathrm{C}(16)-\mathrm{C}(15)-\mathrm{B}(1)$ & $120.9(5)$ \\
\hline $\mathrm{Pt}(1)-\mathrm{C}(1)-\mathrm{H}(1 \mathrm{~B})$ & 109.5 & $\mathrm{C}(15)-\mathrm{C}(16)-\mathrm{C}(17)$ & $121.4(6)$ \\
\hline $\mathrm{H}(1 \mathrm{~A})-\mathrm{C}(1)-\mathrm{H}(1 \mathrm{~B})$ & 109.5 & $\mathrm{C}(15)-\mathrm{C}(16)-\mathrm{H}(16)$ & 119.3 \\
\hline $\mathrm{Pt}(1)-\mathrm{C}(1)-\mathrm{H}(1 \mathrm{C})$ & 109.5 & $\mathrm{C}(17)-\mathrm{C}(16)-\mathrm{H}(16)$ & 119.3 \\
\hline $\mathrm{H}(1 \mathrm{~A})-\mathrm{C}(1)-\mathrm{H}(1 \mathrm{C})$ & 109.5 & $\mathrm{C}(18)-\mathrm{C}(17)-\mathrm{C}(16)$ & $121.7(5)$ \\
\hline $\mathrm{H}(1 \mathrm{~B})-\mathrm{C}(1)-\mathrm{H}(1 \mathrm{C})$ & 109.5 & $\mathrm{C}(18)-\mathrm{C}(17)-\mathrm{H}(17)$ & 119.2 \\
\hline $\mathrm{Pt}(1)-\mathrm{C}(2)-\mathrm{H}(2 \mathrm{~A})$ & 109.5 & $\mathrm{C}(16)-\mathrm{C}(17)-\mathrm{H}(17)$ & 119.2 \\
\hline $\mathrm{Pt}(1)-\mathrm{C}(2)-\mathrm{H}(2 \mathrm{~B})$ & 109.5 & $\mathrm{C}(17)-\mathrm{C}(18)-\mathrm{C}(19)$ & $118.6(5)$ \\
\hline $\mathrm{H}(2 \mathrm{~A})-\mathrm{C}(2)-\mathrm{H}(2 \mathrm{~B})$ & 109.5 & $\mathrm{C}(17)-\mathrm{C}(18)-\mathrm{H}(18)$ & 120.7 \\
\hline $\mathrm{Pt}(1)-\mathrm{C}(2)-\mathrm{H}(2 \mathrm{C})$ & 109.5 & $\mathrm{C}(19)-\mathrm{C}(18)-\mathrm{H}(18)$ & 120.7 \\
\hline $\mathrm{H}(2 \mathrm{~A})-\mathrm{C}(2)-\mathrm{H}(2 \mathrm{C})$ & 109.5 & $\mathrm{C}(18)-\mathrm{C}(19)-\mathrm{C}(20)$ & $119.8(6)$ \\
\hline $\mathrm{H}(2 \mathrm{~B})-\mathrm{C}(2)-\mathrm{H}(2 \mathrm{C})$ & 109.5 & $\mathrm{C}(18)-\mathrm{C}(19)-\mathrm{H}(19)$ & 120.1 \\
\hline $\mathrm{C}(23)-\mathrm{P}(1)-\mathrm{C}(33)$ & $109.43(17)$ & $\mathrm{C}(20)-\mathrm{C}(19)-\mathrm{H}(19)$ & 120.1 \\
\hline $\mathrm{C}(23)-\mathrm{P}(1)-\mathrm{C}(27)$ & $100.84(17)$ & $C(15)-C(20)-C(19)$ & $124.5(5)$ \\
\hline $\mathrm{C}(33)-\mathrm{P}(1)-\mathrm{C}(27)$ & $101.64(16)$ & $\mathrm{C}(15)-\mathrm{C}(20)-\mathrm{H}(20)$ & 117.8 \\
\hline
\end{tabular}




\begin{tabular}{|c|c|c|c|}
\hline $\mathrm{C}(19)-\mathrm{C}(20)-\mathrm{H}(20)$ & 117.8 & $\mathrm{C}(45)-\mathrm{B}(2)-\mathrm{C}(51)$ & $110.8(3)$ \\
\hline $\mathrm{C}(26)-\mathrm{C}(21)-\mathrm{C}(22)$ & 115.1(3) & $\mathrm{C}(39)-\mathrm{B}(2)-\mathrm{C}(51)$ & $110.1(3)$ \\
\hline $\mathrm{C}(26)-\mathrm{C}(21)-\mathrm{B}(1)$ & $125.5(4)$ & $\mathrm{C}(45)-\mathrm{B}(2)-\mathrm{C}(57)$ & $110.0(3)$ \\
\hline $\mathrm{C}(22)-\mathrm{C}(21)-\mathrm{B}(1)$ & 119.4(3) & $\mathrm{C}(39)-\mathrm{B}(2)-\mathrm{C}(57)$ & $114.4(3)$ \\
\hline $\mathrm{C}(23)-\mathrm{C}(22)-\mathrm{C}(21)$ & $124.0(3)$ & $\mathrm{C}(51)-\mathrm{B}(2)-\mathrm{C}(57)$ & 104.6(3) \\
\hline $\mathrm{C}(23)-\mathrm{C}(22)-\mathrm{H}(22)$ & 118.0 & $\mathrm{C}(59)-\mathrm{P}(2)-\mathrm{C}(69)$ & $101.54(17)$ \\
\hline $\mathrm{C}(21)-\mathrm{C}(22)-\mathrm{H}(22)$ & 118.0 & $\mathrm{C}(59)-\mathrm{P}(2)-\mathrm{C}(63)$ & $104.23(17)$ \\
\hline $\mathrm{C}(24)-\mathrm{C}(23)-\mathrm{C}(22)$ & $118.5(3)$ & $\mathrm{C}(69)-\mathrm{P}(2)-\mathrm{C}(63)$ & $100.53(17)$ \\
\hline $\mathrm{C}(24)-\mathrm{C}(23)-\mathrm{P}(1)$ & $118.5(3)$ & $\mathrm{C}(59)-\mathrm{P}(2)-\mathrm{Pt}(1)$ & $117.42(12)$ \\
\hline $\mathrm{C}(22)-\mathrm{C}(23)-\mathrm{P}(1)$ & $122.7(3)$ & $\mathrm{C}(69)-\mathrm{P}(2)-\mathrm{Pt}(1)$ & $120.45(11)$ \\
\hline $\mathrm{C}(25)-\mathrm{C}(24)-\mathrm{C}(23)$ & 119.3(3) & $\mathrm{C}(63)-\mathrm{P}(2)-\mathrm{Pt}(1)$ & $110.31(13)$ \\
\hline $\mathrm{C}(25)-\mathrm{C}(24)-\mathrm{H}(24)$ & 120.3 & $\mathrm{C}(40)-\mathrm{C}(39)-\mathrm{C}(44)$ & $115.0(4)$ \\
\hline $\mathrm{C}(23)-\mathrm{C}(24)-\mathrm{H}(24)$ & 120.3 & $\mathrm{C}(40)-\mathrm{C}(39)-\mathrm{B}(2)$ & 126.1(3) \\
\hline $\mathrm{C}(24)-\mathrm{C}(25)-\mathrm{C}(26)$ & $120.6(3)$ & $\mathrm{C}(44)-\mathrm{C}(39)-\mathrm{B}(2)$ & $118.9(3)$ \\
\hline $\mathrm{C}(24)-\mathrm{C}(25)-\mathrm{H}(25)$ & 119.7 & $\mathrm{C}(41)-\mathrm{C}(40)-\mathrm{C}(39)$ & $122.8(4)$ \\
\hline $\mathrm{C}(26)-\mathrm{C}(25)-\mathrm{H}(25)$ & 119.7 & $\mathrm{C}(41)-\mathrm{C}(40)-\mathrm{H}(40)$ & 118.6 \\
\hline $\mathrm{C}(25)-\mathrm{C}(26)-\mathrm{C}(21)$ & $122.4(4)$ & $\mathrm{C}(39)-\mathrm{C}(40)-\mathrm{H}(40)$ & 118.6 \\
\hline $\mathrm{C}(25)-\mathrm{C}(26)-\mathrm{H}(26)$ & 118.8 & $\mathrm{C}(42)-\mathrm{C}(41)-\mathrm{C}(40)$ & $120.5(4)$ \\
\hline $\mathrm{C}(21)-\mathrm{C}(26)-\mathrm{H}(26)$ & 118.8 & $\mathrm{C}(42)-\mathrm{C}(41)-\mathrm{H}(41)$ & 119.8 \\
\hline $\mathrm{C}(28)-\mathrm{C}(27)-\mathrm{C}(32)$ & 118.1(3) & $\mathrm{C}(40)-\mathrm{C}(41)-\mathrm{H}(41)$ & 119.8 \\
\hline $\mathrm{C}(28)-\mathrm{C}(27)-\mathrm{P}(1)$ & $120.2(3)$ & $\mathrm{C}(41)-\mathrm{C}(42)-\mathrm{C}(43)$ & $118.2(4)$ \\
\hline $\mathrm{C}(32)-\mathrm{C}(27)-\mathrm{P}(1)$ & $121.6(3)$ & $\mathrm{C}(41)-\mathrm{C}(42)-\mathrm{H}(42)$ & 120.9 \\
\hline $\mathrm{C}(27)-\mathrm{C}(28)-\mathrm{C}(29)$ & $121.0(4)$ & $\mathrm{C}(43)-\mathrm{C}(42)-\mathrm{H}(42)$ & 120.9 \\
\hline $\mathrm{C}(27)-\mathrm{C}(28)-\mathrm{H}(28)$ & 119.5 & $\mathrm{C}(44)-\mathrm{C}(43)-\mathrm{C}(42)$ & $121.2(4)$ \\
\hline $\mathrm{C}(29)-\mathrm{C}(28)-\mathrm{H}(28)$ & 119.5 & $\mathrm{C}(44)-\mathrm{C}(43)-\mathrm{H}(43)$ & 119.4 \\
\hline $\mathrm{C}(30)-\mathrm{C}(29)-\mathrm{C}(28)$ & $120.2(4)$ & $\mathrm{C}(42)-\mathrm{C}(43)-\mathrm{H}(43)$ & 119.4 \\
\hline $\mathrm{C}(30)-\mathrm{C}(29)-\mathrm{H}(29)$ & 119.9 & $\mathrm{C}(43)-\mathrm{C}(44)-\mathrm{C}(39)$ & $122.3(4)$ \\
\hline $\mathrm{C}(28)-\mathrm{C}(29)-\mathrm{H}(29)$ & 119.9 & $\mathrm{C}(43)-\mathrm{C}(44)-\mathrm{H}(44)$ & 118.9 \\
\hline $\mathrm{C}(31)-\mathrm{C}(30)-\mathrm{C}(29)$ & $119.2(4)$ & $\mathrm{C}(39)-\mathrm{C}(44)-\mathrm{H}(44)$ & 118.9 \\
\hline $\mathrm{C}(31)-\mathrm{C}(30)-\mathrm{H}(30)$ & 120.4 & $\mathrm{C}(50)-\mathrm{C}(45)-\mathrm{C}(46)$ & $115.1(4)$ \\
\hline $\mathrm{C}(29)-\mathrm{C}(30)-\mathrm{H}(30)$ & 120.4 & $\mathrm{C}(50)-\mathrm{C}(45)-\mathrm{B}(2)$ & $124.6(3)$ \\
\hline $\mathrm{C}(30)-\mathrm{C}(31)-\mathrm{C}(32)$ & $120.5(4)$ & $\mathrm{C}(46)-\mathrm{C}(45)-\mathrm{B}(2)$ & $120.3(3)$ \\
\hline $\mathrm{C}(30)-\mathrm{C}(31)-\mathrm{H}(31)$ & 119.8 & $\mathrm{C}(47)-\mathrm{C}(46)-\mathrm{C}(45)$ & $123.0(4)$ \\
\hline $\mathrm{C}(32)-\mathrm{C}(31)-\mathrm{H}(31)$ & 119.8 & $\mathrm{C}(47)-\mathrm{C}(46)-\mathrm{H}(46)$ & 118.5 \\
\hline $\mathrm{C}(31)-\mathrm{C}(32)-\mathrm{C}(27)$ & 121.1(4) & $\mathrm{C}(45)-\mathrm{C}(46)-\mathrm{H}(46)$ & 118.5 \\
\hline $\mathrm{C}(31)-\mathrm{C}(32)-\mathrm{H}(32)$ & 119.5 & $\mathrm{C}(46)-\mathrm{C}(47)-\mathrm{C}(48)$ & $120.1(4)$ \\
\hline $\mathrm{C}(27)-\mathrm{C}(32)-\mathrm{H}(32)$ & 119.5 & $\mathrm{C}(46)-\mathrm{C}(47)-\mathrm{H}(47)$ & 120.0 \\
\hline $\mathrm{C}(34)-\mathrm{C}(33)-\mathrm{C}(38)$ & $118.8(3)$ & $\mathrm{C}(48)-\mathrm{C}(47)-\mathrm{H}(47)$ & 120.0 \\
\hline $\mathrm{C}(34)-\mathrm{C}(33)-\mathrm{P}(1)$ & $126.6(3)$ & $\mathrm{C}(49)-\mathrm{C}(48)-\mathrm{C}(47)$ & $118.6(4)$ \\
\hline $\mathrm{C}(38)-\mathrm{C}(33)-\mathrm{P}(1)$ & $114.6(3)$ & $\mathrm{C}(49)-\mathrm{C}(48)-\mathrm{H}(48)$ & 120.7 \\
\hline $\mathrm{C}(33)-\mathrm{C}(34)-\mathrm{C}(35)$ & $119.8(4)$ & $\mathrm{C}(47)-\mathrm{C}(48)-\mathrm{H}(48)$ & 120.7 \\
\hline $\mathrm{C}(33)-\mathrm{C}(34)-\mathrm{H}(34)$ & 120.1 & $\mathrm{C}(48)-\mathrm{C}(49)-\mathrm{C}(50)$ & $120.5(4)$ \\
\hline $\mathrm{C}(35)-\mathrm{C}(34)-\mathrm{H}(34)$ & 120.1 & $\mathrm{C}(48)-\mathrm{C}(49)-\mathrm{H}(49)$ & 119.7 \\
\hline$C(36)-C(35)-C(34)$ & $120.7(4)$ & $\mathrm{C}(50)-\mathrm{C}(49)-\mathrm{H}(49)$ & 119.7 \\
\hline $\mathrm{C}(36)-\mathrm{C}(35)-\mathrm{H}(35)$ & 119.7 & $C(49)-C(50)-C(45)$ & $122.6(4)$ \\
\hline $\mathrm{C}(34)-\mathrm{C}(35)-\mathrm{H}(35)$ & 119.7 & $\mathrm{C}(49)-\mathrm{C}(50)-\mathrm{H}(50)$ & 118.7 \\
\hline $\mathrm{C}(35)-\mathrm{C}(36)-\mathrm{C}(37)$ & $120.1(4)$ & $\mathrm{C}(45)-\mathrm{C}(50)-\mathrm{H}(50)$ & 118.7 \\
\hline $\mathrm{C}(35)-\mathrm{C}(36)-\mathrm{H}(36)$ & 119.9 & $\mathrm{C}(56)-\mathrm{C}(51)-\mathrm{C}(52)$ & $115.4(4)$ \\
\hline $\mathrm{C}(37)-\mathrm{C}(36)-\mathrm{H}(36)$ & 119.9 & $\mathrm{C}(56)-\mathrm{C}(51)-\mathrm{B}(2)$ & $124.8(3)$ \\
\hline $\mathrm{C}(38)-\mathrm{C}(37)-\mathrm{C}(36)$ & $119.5(4)$ & $\mathrm{C}(52)-\mathrm{C}(51)-\mathrm{B}(2)$ & $119.8(3)$ \\
\hline $\mathrm{C}(38)-\mathrm{C}(37)-\mathrm{H}(37)$ & 120.2 & $C(53)-C(52)-C(51)$ & $122.7(4)$ \\
\hline $\mathrm{C}(36)-\mathrm{C}(37)-\mathrm{H}(37)$ & 120.2 & $\mathrm{C}(53)-\mathrm{C}(52)-\mathrm{H}(52)$ & 118.6 \\
\hline $\mathrm{C}(37)-\mathrm{C}(38)-\mathrm{C}(33)$ & $121.0(4)$ & $\mathrm{C}(51)-\mathrm{C}(52)-\mathrm{H}(52)$ & 118.6 \\
\hline $\mathrm{C}(37)-\mathrm{C}(38)-\mathrm{H}(38)$ & 119.5 & $\mathrm{C}(52)-\mathrm{C}(53)-\mathrm{C}(54)$ & $119.9(4)$ \\
\hline $\mathrm{C}(33)-\mathrm{C}(38)-\mathrm{H}(38)$ & 119.5 & $\mathrm{C}(52)-\mathrm{C}(53)-\mathrm{H}(53)$ & 120.1 \\
\hline $\mathrm{C}(45)-\mathrm{B}(2)-\mathrm{C}(39)$ & 107.1(3) & $\mathrm{C}(54)-\mathrm{C}(53)-\mathrm{H}(53)$ & 120.1 \\
\hline
\end{tabular}




$\begin{array}{ll}\mathrm{C}(55)-\mathrm{C}(54)-\mathrm{C}(53) & 119.2(4) \\ \mathrm{C}(55)-\mathrm{C}(54)-\mathrm{H}(54) & 120.4 \\ \mathrm{C}(53)-\mathrm{C}(54)-\mathrm{H}(54) & 120.4 \\ \mathrm{C}(54)-\mathrm{C}(55)-\mathrm{C}(56) & 120.1(4) \\ \mathrm{C}(54)-\mathrm{C}(55)-\mathrm{H}(55) & 119.9 \\ \mathrm{C}(56)-\mathrm{C}(55)-\mathrm{H}(55) & 119.9 \\ \mathrm{C}(55)-\mathrm{C}(56)-\mathrm{C}(51) & 122.7(4) \\ \mathrm{C}(55)-\mathrm{C}(56)-\mathrm{H}(56) & 118.7 \\ \mathrm{C}(51)-\mathrm{C}(56)-\mathrm{H}(56) & 118.7 \\ \mathrm{C}(58)-\mathrm{C}(57)-\mathrm{C}(62) & 114.7(3) \\ \mathrm{C}(58)-\mathrm{C}(57)-\mathrm{B}(2) & 120.7(3) \\ \mathrm{C}(62)-\mathrm{C}(57)-\mathrm{B}(2) & 124.3(3) \\ \mathrm{C}(59)-\mathrm{C}(58)-\mathrm{C}(57) & 123.5(3) \\ \mathrm{C}(59)-\mathrm{C}(58)-\mathrm{H}(58) & 118.2 \\ \mathrm{C}(57)-\mathrm{C}(58)-\mathrm{H}(58) & 118.2 \\ \mathrm{C}(60)-\mathrm{C}(59)-\mathrm{C}(58) & 119.0(3) \\ \mathrm{C}(60)-\mathrm{C}(59)-\mathrm{P}(2) & 119.3(3) \\ \mathrm{C}(58)-\mathrm{C}(59)-\mathrm{P}(2) & 121.6(3) \\ \mathrm{C}(61)-\mathrm{C}(60)-\mathrm{C}(59) & 119.2(3) \\ \mathrm{C}(61)-\mathrm{C}(60)-\mathrm{H}(60) & 120.4 \\ \mathrm{C}(59)-\mathrm{C}(60)-\mathrm{H}(60) & 120.4 \\ \mathrm{C}(62)-\mathrm{C}(61)-\mathrm{C}(60) & 120.4(3) \\ \mathrm{C}(62)-\mathrm{C}(61)-\mathrm{H}(61) & 119.8 \\ \mathrm{C}(60)-\mathrm{C}(61)-\mathrm{H}(61) & 119.8 \\ \mathrm{C}(61)-\mathrm{C}(62)-\mathrm{C}(57) & 123.1(3) \\ \mathrm{C}(61)-\mathrm{C}(62)-\mathrm{H}(62) & 118.4 \\ \mathrm{C}(57)-\mathrm{C}(62)-\mathrm{H}(62) & 118.4 \\ \mathrm{C}(68)-\mathrm{C}(63)-\mathrm{C}(64) & 118.8(4) \\ \mathrm{C}(68)-\mathrm{C}(63)-\mathrm{P}(2) & 117.7(3) \\ \mathrm{C}(64)-\mathrm{C}(63)-\mathrm{P}(2) & 123.3(3) \\ \mathrm{C}(63)-\mathrm{C}(64)-\mathrm{C}(65) & 120.0(4) \\ \mathrm{C}(63)-\mathrm{C}(64)-\mathrm{H}(64) & 120.0 \\ \mathrm{C}(65)-\mathrm{C}(64)-\mathrm{H}(64) & 120.0 \\ \mathrm{C}(66)-\mathrm{C}(65)-\mathrm{C}(64) & 120.6(5) \\ \mathrm{C}(66)-\mathrm{C}(65)-\mathrm{H}(65) & 119.7 \\ \mathrm{C}(64)-\mathrm{C}(65)-\mathrm{H}(65) & 119.7 \\ \mathrm{C}(67)-\mathrm{C}(66)-\mathrm{C}(65) & 119.6(4) \\ \mathrm{C}(67)-\mathrm{C}(66)-\mathrm{H}(66) & 120.2 \\ \mathrm{C}(65)-\mathrm{C}(66)-\mathrm{H}(66) & 120.2 \\ \mathrm{C}(66)-\mathrm{C}(67)-\mathrm{C}(68) & 120.2(5) \\ \mathrm{C}(66)-\mathrm{C}(67)-\mathrm{H}(67) & 119.9 \\ \mathrm{C}(68)-\mathrm{C}(67)-\mathrm{H}(67) & 119.9 \\ \mathrm{C}(63)-\mathrm{C}(68)-\mathrm{C}(67) & 120.8(4) \\ \mathrm{C}(63)-\mathrm{C}(68)-\mathrm{H}(68) & 119.6 \\ \mathrm{C}(67)-\mathrm{C}(68)-\mathrm{H}(68) & 119.6 \\ \mathrm{C}(70)-\mathrm{C}(69)-\mathrm{C}(74) & 118.8(3) \\ \mathrm{C}(70)-\mathrm{C}(69)-\mathrm{P}(2) & 121.9(3) \\ \mathrm{C}(74)-\mathrm{C}(69)-\mathrm{P}(2) & 119.3(3) \\ \mathrm{C}(69)-\mathrm{C}(70)-\mathrm{C}(71) & 120.5(4) \\ \mathrm{C}(69)-\mathrm{C}(70)-\mathrm{H}(70) & 119.8 \\ \mathrm{C}(71)-\mathrm{C}(70)-\mathrm{H}(70) & 119.8 \\ \mathrm{C}(72)-\mathrm{C}(71)-\mathrm{C}(70) & 120.4(4) \\ \mathrm{C}(72)-\mathrm{C}(71)-\mathrm{H}(71) & 119.8 \\ \mathrm{C}(70)-\mathrm{C}(71)-\mathrm{H}(71) & 119.8 \\ \mathrm{C}(71)-\mathrm{C}(72)-\mathrm{C}(73) & 120.0(4) \\ \mathrm{C}(71)-\mathrm{C}(72)-\mathrm{H}(72) & 120.0 \\ & \end{array}$

$\begin{array}{ll} & \\ \mathrm{C}(73)-\mathrm{C}(72)-\mathrm{H}(72) & 120.0 \\ \mathrm{C}(74)-\mathrm{C}(73)-\mathrm{C}(72) & 119.5(4) \\ \mathrm{C}(74)-\mathrm{C}(73)-\mathrm{H}(73) & 120.2 \\ \mathrm{C}(72)-\mathrm{C}(73)-\mathrm{H}(73) & 120.2 \\ \mathrm{C}(73)-\mathrm{C}(74)-\mathrm{C}(69) & 120.8(3) \\ \mathrm{C}(73)-\mathrm{C}(74)-\mathrm{H}(74) & 119.6 \\ \mathrm{C}(69)-\mathrm{C}(74)-\mathrm{H}(74) & 119.6 \\ \mathrm{C}(75)-\mathrm{N}(1)-\mathrm{C}(78) & 101.7(3) \\ \mathrm{C}(75)-\mathrm{N}(1)-\mathrm{C}(79) & 113.2(3) \\ \mathrm{C}(78)-\mathrm{N}(1)-\mathrm{C}(79) & 110.6(3) \\ \mathrm{C}(75)-\mathrm{N}(1)-\mathrm{C}(82) & 113.5(4) \\ \mathrm{C}(78)-\mathrm{N}(1)-\mathrm{C}(82) & 112.9(3) \\ \mathrm{C}(79)-\mathrm{N}(1)-\mathrm{C}(82) & 105.0(3) \\ \mathrm{N}(1)-\mathrm{C}(75)-\mathrm{C}(76) & 106.1(4) \\ \mathrm{N}(1)-\mathrm{C}(75)-\mathrm{H}(75 \mathrm{~A}) & 110.5 \\ \mathrm{C}(76)-\mathrm{C}(75)-\mathrm{H}(75 \mathrm{~A}) & 110.5 \\ \mathrm{~N}(1)-\mathrm{C}(75)-\mathrm{H}(75 \mathrm{~B}) & 110.5 \\ \mathrm{C}(76)-\mathrm{C}(75)-\mathrm{H}(75 \mathrm{~B}) & 110.5 \\ \mathrm{H}(75 \mathrm{~A})-\mathrm{C}(75)-\mathrm{H}(75 \mathrm{~B}) & 108.7 \\ \mathrm{C}(75)-\mathrm{C}(76)-\mathrm{C}(77) & 104.3(4) \\ \mathrm{C}(75)-\mathrm{C}(76)-\mathrm{H}(76 \mathrm{~A}) & 110.9 \\ \mathrm{C}(77)-\mathrm{C}(76)-\mathrm{H}(76 \mathrm{~A}) & 110.9 \\ \mathrm{C}(75)-\mathrm{C}(76)-\mathrm{H}(76 \mathrm{~B}) & 110.9 \\ \mathrm{C}(77)-\mathrm{C}(76)-\mathrm{H}(76 \mathrm{~B}) & 110.9 \\ \mathrm{H}(76 \mathrm{~A})-\mathrm{C}(76)-\mathrm{H}(76 \mathrm{~B}) & 108.9 \\ \mathrm{C}(78)-\mathrm{C}(77)-\mathrm{C}(76) & 105.9(4) \\ \mathrm{C}(78)-\mathrm{C}(77)-\mathrm{H}(77 \mathrm{~A}) & 110.5 \\ \mathrm{C}(76)-\mathrm{C}(77)-\mathrm{H}(77 \mathrm{~A}) & 110.5 \\ \mathrm{C}(78)-\mathrm{C}(77)-\mathrm{H}(77 \mathrm{~B}) & 110.6 \\ \mathrm{C}(76)-\mathrm{C}(77)-\mathrm{H}(77 \mathrm{~B}) & 110.5 \\ \mathrm{H}(77 \mathrm{~A})-\mathrm{C}(77)-\mathrm{H}(77 \mathrm{~B}) & 108.7 \\ \mathrm{~N}(1)-\mathrm{C}(78)-\mathrm{C}(77) & 104.4(4) \\ \mathrm{N}(1)-\mathrm{C}(78)-\mathrm{H}(78 \mathrm{~A}) & 110.9 \\ \mathrm{C}(77)-\mathrm{C}(78)-\mathrm{H}(78 \mathrm{~A}) & 110.9 \\ \mathrm{~N}(1)-\mathrm{C}(78)-\mathrm{H}(78 \mathrm{~B}) & 110.9 \\ \mathrm{C}(77)-\mathrm{C}(78)-\mathrm{H}(78 \mathrm{~B}) & 110.9 \\ \mathrm{H}(78 \mathrm{~A})-\mathrm{C}(78)-\mathrm{H}(78 \mathrm{~B}) & 108.9 \\ \mathrm{~N}(1)-\mathrm{C}(79)-\mathrm{C}(80) & 103.3(3) \\ \mathrm{N}(1)-\mathrm{C}(79)-\mathrm{H}(79 \mathrm{~A}) & 111.1 \\ \mathrm{C}(80)-\mathrm{C}(79)-\mathrm{H}(79 \mathrm{~A}) & 111.1 \\ \mathrm{~N}(1)-\mathrm{C}(79)-\mathrm{H}(79 \mathrm{~B}) & 111.1 \\ \mathrm{C}(80)-\mathrm{C}(79)-\mathrm{H}(79 \mathrm{~B}) & 111.1 \\ \mathrm{H}(79 \mathrm{~A})-\mathrm{C}(79)-\mathrm{H}(79 \mathrm{~B}) & 109.1 \\ \mathrm{C}(79)-\mathrm{C}(80)-\mathrm{C}(81) & 103.1(4) \\ \mathrm{C}(79)-\mathrm{C}(80)-\mathrm{H}(80 \mathrm{~A}) & 111.1 \\ \mathrm{C}(81)-\mathrm{C}(80)-\mathrm{H}(80 \mathrm{~A}) & 111.1 \\ \mathrm{C}(79)-\mathrm{C}(80)-\mathrm{H}(80 \mathrm{~B}) & 111.1 \\ \mathrm{C}(81)-\mathrm{C}(80)-\mathrm{H}(80 \mathrm{~B}) & 111.1 \\ \mathrm{H}(80 \mathrm{~A})-\mathrm{C}(80)-\mathrm{H}(80 \mathrm{~B}) & 109.1 \\ \mathrm{C}(82)-\mathrm{C}(81)-\mathrm{C}(80) & 106.1(4) \\ \mathrm{C}(82)-\mathrm{C}(81)-\mathrm{H}(81 \mathrm{~A}) & 110.5 \\ \mathrm{C}(80)-\mathrm{C}(81)-\mathrm{H}(81 \mathrm{~A}) & 110.5 \\ \mathrm{C}(82)-\mathrm{C}(81)-\mathrm{H}(81 \mathrm{~B}) & 110.5 \\ \mathrm{C}(80)-\mathrm{C}(81)-\mathrm{H}(81 \mathrm{~B}) & 110.5 \\ \mathrm{H}(81 \mathrm{~A})-\mathrm{C}(81)-\mathrm{H}(81 \mathrm{~B}) & 108.7 \\ \mathrm{C}(81)-\mathrm{C}(82)-\mathrm{N}(1) & 105.9(4) \\ & \end{array}$




\begin{tabular}{|c|c|c|c|}
\hline $\mathrm{C}(81)-\mathrm{C}(82)-\mathrm{H}(82 \mathrm{~A})$ & 110.5 & $\mathrm{~N}(3)-\mathrm{C}(90)-\mathrm{H}(90 \mathrm{~B})$ & 111.0 \\
\hline $\mathrm{N}(1)-\mathrm{C}(82)-\mathrm{H}(82 \mathrm{~A})$ & 110.5 & $\mathrm{C}(89)-\mathrm{C}(90)-\mathrm{H}(90 \mathrm{~B})$ & 111.0 \\
\hline $\mathrm{C}(81)-\mathrm{C}(82)-\mathrm{H}(82 \mathrm{~B})$ & 110.6 & H(90A)-C(90)-H(90B) & 109.0 \\
\hline $\mathrm{N}(1)-\mathrm{C}(82)-\mathrm{H}(82 \mathrm{~B})$ & 110.6 & $\mathrm{C}(90)-\mathrm{N}(3)-\mathrm{C}(87)$ & $102.3(3)$ \\
\hline $\mathrm{H}(82 \mathrm{~A})-\mathrm{C}(82)-\mathrm{H}(82 \mathrm{~B})$ & 108.7 & $\mathrm{C}(90)-\mathrm{N}(3)-\mathrm{C}(83)$ & $111.2(3)$ \\
\hline $\mathrm{C}(94)-\mathrm{N}(2)-\mathrm{C}(98)$ & $113.7(3)$ & $\mathrm{C}(87)-\mathrm{N}(3)-\mathrm{C}(83)$ & $111.0(3)$ \\
\hline $\mathrm{C}(94)-\mathrm{N}(2)-\mathrm{C}(95)$ & 113.3(3) & $\mathrm{C}(90)-\mathrm{N}(3)-\mathrm{C}(86)$ & $114.4(3)$ \\
\hline $\mathrm{C}(98)-\mathrm{N}(2)-\mathrm{C}(95)$ & 102.3(3) & $\mathrm{C}(87)-\mathrm{N}(3)-\mathrm{C}(86)$ & $112.5(3)$ \\
\hline $\mathrm{C}(94)-\mathrm{N}(2)-\mathrm{C}(91)$ & $102.9(3)$ & $\mathrm{C}(83)-\mathrm{N}(3)-\mathrm{C}(86)$ & $105.7(3)$ \\
\hline $\mathrm{C}(98)-\mathrm{N}(2)-\mathrm{C}(91)$ & $113.9(3)$ & $\mathrm{N}(2)-\mathrm{C}(91)-\mathrm{C}(92)$ & $104.1(3)$ \\
\hline $\mathrm{C}(95)-\mathrm{N}(2)-\mathrm{C}(91)$ & 111.1(3) & $\mathrm{N}(2)-\mathrm{C}(91)-\mathrm{H}(91 \mathrm{~A})$ & 110.9 \\
\hline $\mathrm{C}(84)-\mathrm{C}(83)-\mathrm{N}(3)$ & 104.3(3) & $\mathrm{C}(92)-\mathrm{C}(91)-\mathrm{H}(91 \mathrm{~A})$ & 111.0 \\
\hline $\mathrm{C}(84)-\mathrm{C}(83)-\mathrm{H}(83 \mathrm{~A})$ & 110.9 & $\mathrm{~N}(2)-\mathrm{C}(91)-\mathrm{H}(91 \mathrm{~B})$ & 110.9 \\
\hline $\mathrm{N}(3)-\mathrm{C}(83)-\mathrm{H}(83 \mathrm{~A})$ & 110.9 & $\mathrm{C}(92)-\mathrm{C}(91)-\mathrm{H}(91 \mathrm{~B})$ & 110.9 \\
\hline C(84)-C(83)-H(83B) & 110.9 & H(91A)-C(91)-H(91B) & 109.0 \\
\hline N(3)-C(83)-H(83B) & 110.9 & $\mathrm{C}(91)-\mathrm{C}(92)-\mathrm{C}(93)$ & $105.8(3)$ \\
\hline $\mathrm{H}(83 \mathrm{~A})-\mathrm{C}(83)-\mathrm{H}(83 \mathrm{~B})$ & 108.9 & $\mathrm{C}(91)-\mathrm{C}(92)-\mathrm{H}(92 \mathrm{~A})$ & 110.6 \\
\hline $\mathrm{C}(83)-\mathrm{C}(84)-\mathrm{C}(85)$ & $102.2(4)$ & $\mathrm{C}(93)-\mathrm{C}(92)-\mathrm{H}(92 \mathrm{~A})$ & 110.6 \\
\hline $\mathrm{C}(83)-\mathrm{C}(84)-\mathrm{H}(84 \mathrm{~A})$ & 111.3 & $\mathrm{C}(91)-\mathrm{C}(92)-\mathrm{H}(92 \mathrm{~B})$ & 110.6 \\
\hline $\mathrm{C}(85)-\mathrm{C}(84)-\mathrm{H}(84 \mathrm{~A})$ & 111.3 & $\mathrm{C}(93)-\mathrm{C}(92)-\mathrm{H}(92 \mathrm{~B})$ & 110.6 \\
\hline $\mathrm{C}(83)-\mathrm{C}(84)-\mathrm{H}(84 \mathrm{~B})$ & 111.3 & H(92A)-C(92)-H(92B) & 108.7 \\
\hline $\mathrm{C}(85)-\mathrm{C}(84)-\mathrm{H}(84 \mathrm{~B})$ & 111.3 & $\mathrm{C}(94)-\mathrm{C}(93)-\mathrm{C}(92)$ & $105.2(3)$ \\
\hline H(84A)-C(84)-H(84B) & 109.2 & C(94)-C(93)-H(93A) & 110.7 \\
\hline $\mathrm{C}(86)-\mathrm{C}(85)-\mathrm{C}(84)$ & $104.0(4)$ & $\mathrm{C}(92)-\mathrm{C}(93)-\mathrm{H}(93 \mathrm{~A})$ & 110.7 \\
\hline $\mathrm{C}(86)-\mathrm{C}(85)-\mathrm{H}(85 \mathrm{~A})$ & 110.9 & $\mathrm{C}(94)-\mathrm{C}(93)-\mathrm{H}(93 \mathrm{~B})$ & 110.7 \\
\hline $\mathrm{C}(84)-\mathrm{C}(85)-\mathrm{H}(85 \mathrm{~A})$ & 110.9 & $\mathrm{C}(92)-\mathrm{C}(93)-\mathrm{H}(93 \mathrm{~B})$ & 110.7 \\
\hline $\mathrm{C}(86)-\mathrm{C}(85)-\mathrm{H}(85 \mathrm{~B})$ & 111.0 & H(93A)-C(93)-H(93B) & 108.8 \\
\hline $\mathrm{C}(84)-\mathrm{C}(85)-\mathrm{H}(85 \mathrm{~B})$ & 111.0 & $\mathrm{~N}(2)-\mathrm{C}(94)-\mathrm{C}(93)$ & $103.9(3)$ \\
\hline $\mathrm{H}(85 \mathrm{~A})-\mathrm{C}(85)-\mathrm{H}(85 \mathrm{~B})$ & 109.0 & $\mathrm{~N}(2)-\mathrm{C}(94)-\mathrm{H}(94 \mathrm{~A})$ & 111.0 \\
\hline $\mathrm{C}(85)-\mathrm{C}(86)-\mathrm{N}(3)$ & 106.1(4) & $\mathrm{C}(93)-\mathrm{C}(94)-\mathrm{H}(94 \mathrm{~A})$ & 111.0 \\
\hline $\mathrm{C}(85)-\mathrm{C}(86)-\mathrm{H}(86 \mathrm{~A})$ & 110.5 & N(2)-C(94)-H(94B) & 111.0 \\
\hline $\mathrm{N}(3)-\mathrm{C}(86)-\mathrm{H}(86 \mathrm{~A})$ & 110.5 & $\mathrm{C}(93)-\mathrm{C}(94)-\mathrm{H}(94 \mathrm{~B})$ & 111.0 \\
\hline $\mathrm{C}(85)-\mathrm{C}(86)-\mathrm{H}(86 \mathrm{~B})$ & 110.5 & H(94A)-C(94)-H(94B) & 109.0 \\
\hline N(3)-C(86)-H(86B) & 110.5 & $\mathrm{~N}(2)-\mathrm{C}(95)-\mathrm{C}(96)$ & $104.6(3)$ \\
\hline H(86A)-C(86)-H(86B) & 108.7 & $\mathrm{~N}(2)-\mathrm{C}(95)-\mathrm{H}(95 \mathrm{~A})$ & 110.8 \\
\hline $\mathrm{N}(3)-\mathrm{C}(87)-\mathrm{C}(88)$ & $104.6(3)$ & $\mathrm{C}(96)-\mathrm{C}(95)-\mathrm{H}(95 \mathrm{~A})$ & 110.8 \\
\hline N(3)-C(87)-H(87A) & 110.8 & $\mathrm{~N}(2)-\mathrm{C}(95)-\mathrm{H}(95 \mathrm{~B})$ & 110.8 \\
\hline $\mathrm{C}(88)-\mathrm{C}(87)-\mathrm{H}(87 \mathrm{~A})$ & 110.8 & $\mathrm{C}(96)-\mathrm{C}(95)-\mathrm{H}(95 \mathrm{~B})$ & 110.8 \\
\hline $\mathrm{N}(3)-\mathrm{C}(87)-\mathrm{H}(87 \mathrm{~B})$ & 110.8 & H(95A)-C(95)-H(95B) & 108.9 \\
\hline $\mathrm{C}(88)-\mathrm{C}(87)-\mathrm{H}(87 \mathrm{~B})$ & 110.8 & $\mathrm{C}(95)-\mathrm{C}(96)-\mathrm{C}(97)$ & $104.9(3)$ \\
\hline H(87A)-C(87)-H(87B) & 108.9 & $\mathrm{C}(95)-\mathrm{C}(96)-\mathrm{H}(96 \mathrm{~A})$ & 110.8 \\
\hline $\mathrm{C}(87)-\mathrm{C}(88)-\mathrm{C}(89)$ & $105.7(4)$ & $\mathrm{C}(97)-\mathrm{C}(96)-\mathrm{H}(96 \mathrm{~A})$ & 110.8 \\
\hline $\mathrm{C}(87)-\mathrm{C}(88)-\mathrm{H}(88 \mathrm{~A})$ & 110.6 & $\mathrm{C}(95)-\mathrm{C}(96)-\mathrm{H}(96 \mathrm{~B})$ & 110.8 \\
\hline $\mathrm{C}(89)-\mathrm{C}(88)-\mathrm{H}(88 \mathrm{~A})$ & 110.6 & $\mathrm{C}(97)-\mathrm{C}(96)-\mathrm{H}(96 \mathrm{~B})$ & 110.8 \\
\hline $\mathrm{C}(87)-\mathrm{C}(88)-\mathrm{H}(88 \mathrm{~B})$ & 110.6 & H(96A)-C(96)-H(96B) & 108.8 \\
\hline C(89)-C(88)-H(88B) & 110.6 & $\mathrm{C}(98)-\mathrm{C}(97)-\mathrm{C}(96)$ & $105.6(4)$ \\
\hline $\mathrm{H}(88 \mathrm{~A})-\mathrm{C}(88)-\mathrm{H}(88 \mathrm{~B})$ & 108.7 & $\mathrm{C}(98)-\mathrm{C}(97)-\mathrm{H}(97 \mathrm{~A})$ & 110.6 \\
\hline $\mathrm{C}(90)-\mathrm{C}(89)-\mathrm{C}(88)$ & $104.7(4)$ & $\mathrm{C}(96)-\mathrm{C}(97)-\mathrm{H}(97 \mathrm{~A})$ & 110.6 \\
\hline $\mathrm{C}(90)-\mathrm{C}(89)-\mathrm{H}(89 \mathrm{~A})$ & 110.8 & $\mathrm{C}(98)-\mathrm{C}(97)-\mathrm{H}(97 \mathrm{~B})$ & 110.6 \\
\hline $\mathrm{C}(88)-\mathrm{C}(89)-\mathrm{H}(89 \mathrm{~A})$ & 110.8 & $\mathrm{C}(96)-\mathrm{C}(97)-\mathrm{H}(97 \mathrm{~B})$ & 110.6 \\
\hline C(90)-C(89)-H(89B) & 110.8 & H(97A)-C(97)-H(97B) & 108.8 \\
\hline $\mathrm{C}(88)-\mathrm{C}(89)-\mathrm{H}(89 \mathrm{~B})$ & 110.8 & $\mathrm{C}(97)-\mathrm{C}(98)-\mathrm{N}(2)$ & $104.5(3)$ \\
\hline H(89A)-C(89)-H(89B) & 108.9 & C(97)-C(98)-H(98A) & 110.8 \\
\hline $\mathrm{N}(3)-\mathrm{C}(90)-\mathrm{C}(89)$ & $103.7(3)$ & $\mathrm{N}(2)-\mathrm{C}(98)-\mathrm{H}(98 \mathrm{~A})$ & 110.9 \\
\hline $\mathrm{N}(3)-\mathrm{C}(90)-\mathrm{H}(90 \mathrm{~A})$ & 111.0 & $\mathrm{C}(97)-\mathrm{C}(98)-\mathrm{H}(98 \mathrm{~B})$ & 110.9 \\
\hline $\mathrm{C}(89)-\mathrm{C}(90)-\mathrm{H}(90 \mathrm{~A})$ & 111.0 & $\mathrm{~N}(2)-\mathrm{C}(98)-\mathrm{H}(98 \mathrm{~B})$ & 110.9 \\
\hline
\end{tabular}




$\begin{array}{lc}\text { H(98A)-C(98)-H(98B) } & 108.9 \\ \text { C(101)-C(102)-H(10A) } & 109.5 \\ \text { C(101)-C(102)-H(10B) } & 109.9 \\ \text { H(10A)-C(102)-H(10B) } & 109.5 \\ \text { C(101)-C(102)-H(10C) } & 109.0 \\ \text { H(10A)-C(102)-H(10C) } & 109.5 \\ \text { H(10B)-C(102)-H(10C) } & 109.5 \\ \text { O(1)-C(101)-C(102) } & 150.8(18) \\ \text { O(1)-C(101)-H(10D) } & 97.5 \\ \text { C(102)-C(101)-H(10D) } & 99.7 \\ \text { O(1)-C(101)-H(10E) } & 98.2 \\ \text { C(102)-C(101)-H(10E) } & 100.1 \\ \text { H(10D)-C(101)-H(10E) } & 104.2 \\ \text { C(99)-C(100)-H(10F) } & 111.1 \\ \text { C(99)-C(100)-H(10G) } & 110.7 \\ \text { H(10F)-C(100)-H(10G) } & 108.0 \\ \text { C(99)-C(100)-H(10H) } & 110.5 \\ \text { H(10F)-C(100)-H(10H) } & 108.4 \\ \text { H(10G)-C(100)-H(10H) } & 108.1 \\ \text { C(101)-O(1)-C(99) } & 150.2(16) \\ \text { C(100)-C(99)-O(1) } & 145(5) \\ \text { C(100)-C(99)-H(99A) } & 101.8 \\ \text { O(1)-C(99)-H(99A) } & 100.5 \\ \text { C(100)-C(99)-H(99B) } & 101.1 \\ \text { O(1)-C(99)-H(99B) } & 99.3 \\ \text { H(99A)-C(99)-H(99B) } & 104.7 \\ & \end{array}$


Table 19. Anisotropic displacement parameters $\left(\AA^{2} \times 10^{3}\right)$ for $\left\{\mathrm{ASN}_{2}\left\{\left[\mathrm{Ph}_{3} \mathrm{BP}^{m-}\right.\right.\right.$ $\left.\left.{ }^{\mathrm{Ph} 2}\right]_{2} \mathrm{PtMe}_{2}\right\}$. The anisotropic displacement factor exponent takes the form: $-2 \pi^{2}\left[\mathrm{~h}^{2}\right.$ $\left.\mathrm{a}^{*} \mathrm{U}^{11}+\ldots+2 \mathrm{~h} \mathrm{k} \mathrm{a*} \mathrm{b}^{*} \mathrm{U}^{12}\right]$.

\begin{tabular}{|c|c|c|c|c|c|c|}
\hline & $\mathrm{U}^{11}$ & $\mathrm{U}^{22}$ & $\mathrm{U}^{33}$ & $\mathrm{U}^{23}$ & $\mathrm{U}^{13}$ & $\mathrm{U}^{12}$ \\
\hline$\overline{\mathrm{Pt}(1)}$ & $10(1)$ & 13(1) & 19(1) & $-7(1)$ & $-1(1)$ & $0(1)$ \\
\hline $\mathrm{B}(1)$ & $16(2)$ & $19(2)$ & $25(2)$ & $4(2)$ & $1(2)$ & $1(2)$ \\
\hline $\mathrm{C}(1)$ & $14(2)$ & $18(2)$ & $43(3)$ & $-13(2)$ & $-7(2)$ & $2(2)$ \\
\hline$C(2)$ & $11(2)$ & $19(2)$ & $30(2)$ & $-16(2)$ & 1(2) & $0(1)$ \\
\hline $\mathrm{P}(1)$ & 11(1) & 11(1) & $20(1)$ & $-6(1)$ & 1(1) & $-1(1)$ \\
\hline$C(3)$ & $16(2)$ & $29(2)$ & $25(2)$ & $7(2)$ & $-2(2)$ & $1(2)$ \\
\hline C(4) & $33(3)$ & $50(3)$ & $22(2)$ & $5(2)$ & $-5(2)$ & $-18(2)$ \\
\hline$C(5)$ & $45(3)$ & $63(4)$ & $23(2)$ & $5(2)$ & $-3(2)$ & $-27(3)$ \\
\hline$C(6)$ & $46(3)$ & $40(3)$ & $17(2)$ & $3(2)$ & $-6(2)$ & $-5(2)$ \\
\hline$C(7)$ & $34(3)$ & $19(2)$ & $42(3)$ & $-1(2)$ & $0(2)$ & $0(2)$ \\
\hline $\mathrm{C}(8)$ & $28(2)$ & $18(2)$ & $32(2)$ & $1(2)$ & $5(2)$ & $-1(2)$ \\
\hline $\mathrm{C}(9)$ & $17(2)$ & $23(2)$ & $19(2)$ & $1(2)$ & $-3(2)$ & $-1(2)$ \\
\hline $\mathrm{C}(10)$ & $18(2)$ & $22(2)$ & $28(2)$ & $4(2)$ & $0(2)$ & $1(2)$ \\
\hline $\mathrm{C}(11)$ & $20(2)$ & $22(2)$ & $29(2)$ & $4(2)$ & $-2(2)$ & $-5(2)$ \\
\hline $\mathrm{C}(12)$ & $17(2)$ & $36(2)$ & $19(2)$ & $-2(2)$ & 1(2) & $-7(2)$ \\
\hline $\mathrm{C}(13)$ & $19(2)$ & $32(2)$ & $21(2)$ & $-9(2)$ & $-1(2)$ & $-2(2)$ \\
\hline $\mathrm{C}(14)$ & $20(2)$ & $22(2)$ & $16(2)$ & $-2(2)$ & $-4(2)$ & $-3(2)$ \\
\hline$C(15)$ & $23(2)$ & $19(2)$ & $36(2)$ & $2(2)$ & $13(2)$ & $3(2)$ \\
\hline$C(16)$ & $28(3)$ & $31(3)$ & $66(4)$ & $17(2)$ & $12(2)$ & $3(2)$ \\
\hline $\mathrm{C}(17)$ & $58(4)$ & $21(2)$ & $69(4)$ & $21(3)$ & $36(3)$ & $19(2)$ \\
\hline $\mathrm{C}(18)$ & $73(4)$ & $24(3)$ & $60(4)$ & $-9(3)$ & $36(3)$ & $-10(3)$ \\
\hline $\mathrm{C}(19)$ & $75(4)$ & $27(3)$ & $39(3)$ & $-13(2)$ & $26(3)$ & $-17(3)$ \\
\hline$C(20)$ & $44(3)$ & $17(2)$ & $36(3)$ & $-8(2)$ & $14(2)$ & $-9(2)$ \\
\hline$C(21)$ & $15(2)$ & $11(2)$ & $24(2)$ & $0(1)$ & $2(2)$ & $-3(1)$ \\
\hline $\mathrm{C}(22)$ & $11(2)$ & $11(2)$ & $24(2)$ & $-6(1)$ & $2(1)$ & $-2(1)$ \\
\hline $\mathrm{C}(23)$ & $15(2)$ & $12(2)$ & $16(2)$ & $-4(1)$ & 1(1) & $-5(1)$ \\
\hline$C(24)$ & $11(2)$ & $10(2)$ & $25(2)$ & $-5(1)$ & 1(1) & $-1(1)$ \\
\hline$C(25)$ & $15(2)$ & $17(2)$ & $23(2)$ & $-7(2)$ & $4(2)$ & $-1(1)$ \\
\hline$C(26)$ & $19(2)$ & $16(2)$ & $20(2)$ & $-3(1)$ & $1(2)$ & $-1(1)$ \\
\hline $\mathrm{C}(27)$ & $12(2)$ & $14(2)$ & $29(2)$ & $-8(2)$ & $-4(2)$ & $2(1)$ \\
\hline$C(28)$ & $17(2)$ & $18(2)$ & $30(2)$ & $-10(2)$ & $2(2)$ & $-3(2)$ \\
\hline$C(29)$ & $19(2)$ & $19(2)$ & $39(2)$ & $-8(2)$ & $3(2)$ & $-7(2)$ \\
\hline $\mathrm{C}(30)$ & $21(2)$ & $15(2)$ & $45(3)$ & $-14(2)$ & $-2(2)$ & $-4(2)$ \\
\hline $\mathrm{C}(31)$ & $20(2)$ & $23(2)$ & $38(2)$ & $-18(2)$ & $3(2)$ & $-3(2)$ \\
\hline$C(32)$ & $17(2)$ & $19(2)$ & $29(2)$ & $-10(2)$ & $1(2)$ & $-1(2)$ \\
\hline$C(33)$ & $12(2)$ & $11(2)$ & $25(2)$ & $-9(1)$ & $4(1)$ & $-2(1)$ \\
\hline $\mathrm{C}(34)$ & $17(2)$ & $13(2)$ & $25(2)$ & $-7(2)$ & $1(2)$ & $-1(1)$ \\
\hline $\mathrm{C}(35)$ & $13(2)$ & $18(2)$ & $33(2)$ & $-11(2)$ & $-1(2)$ & $-1(1)$ \\
\hline$C(36)$ & $13(2)$ & $24(2)$ & $34(2)$ & $-7(2)$ & $6(2)$ & $-6(2)$ \\
\hline C(37) & $23(2)$ & $24(2)$ & $21(2)$ & $-6(2)$ & $6(2)$ & $-5(2)$ \\
\hline $\mathrm{C}(38)$ & $14(2)$ & $17(2)$ & $26(2)$ & $-5(2)$ & $1(2)$ & $-2(1)$ \\
\hline $\mathrm{B}(2)$ & $14(2)$ & $13(2)$ & $22(2)$ & $-6(2)$ & $-2(2)$ & $1(2)$ \\
\hline $\mathrm{P}(2)$ & 11(1) & $12(1)$ & $14(1)$ & $-4(1)$ & $0(1)$ & $-1(1)$ \\
\hline C(39) & $20(2)$ & $16(2)$ & $19(2)$ & $-4(2)$ & $-1(2)$ & $-3(1)$ \\
\hline $\mathrm{C}(40)$ & $18(2)$ & $16(2)$ & $22(2)$ & $-6(2)$ & $-1(2)$ & $-2(1)$ \\
\hline $\mathrm{C}(41)$ & $21(2)$ & $22(2)$ & $28(2)$ & $-7(2)$ & $-2(2)$ & $2(2)$ \\
\hline
\end{tabular}




\begin{tabular}{|c|c|c|c|c|c|c|}
\hline$C(42)$ & $17(2)$ & $32(2)$ & $30(2)$ & $-8(2)$ & $-3(2)$ & $-3(2)$ \\
\hline $\mathrm{C}(43)$ & $28(2)$ & $25(2)$ & $28(2)$ & $-6(2)$ & $-4(2)$ & $-12(2)$ \\
\hline$C(44)$ & $24(2)$ & $16(2)$ & $26(2)$ & $-7(2)$ & $-2(2)$ & $-5(2)$ \\
\hline$C(45)$ & $18(2)$ & $17(2)$ & $20(2)$ & $-9(2)$ & $-2(2)$ & $-2(1)$ \\
\hline C(46) & $27(2)$ & $20(2)$ & $22(2)$ & $-6(2)$ & $-3(2)$ & $-1(2)$ \\
\hline $\mathrm{C}(47)$ & $36(2)$ & $32(2)$ & $18(2)$ & $-7(2)$ & $4(2)$ & $-7(2)$ \\
\hline$C(48)$ & $34(2)$ & $33(2)$ & $30(2)$ & $-18(2)$ & $9(2)$ & $-3(2)$ \\
\hline C(49) & $38(3)$ & $19(2)$ & $34(2)$ & $-14(2)$ & $7(2)$ & $-1(2)$ \\
\hline $\mathrm{C}(50)$ & $28(2)$ & $17(2)$ & $27(2)$ & $-9(2)$ & $2(2)$ & $-4(2)$ \\
\hline $\mathrm{C}(51)$ & $20(2)$ & $12(2)$ & $19(2)$ & $-8(1)$ & $3(2)$ & $-3(1)$ \\
\hline $\mathrm{C}(52)$ & $20(2)$ & $17(2)$ & $23(2)$ & $-7(2)$ & 1(2) & $-4(2)$ \\
\hline $\mathrm{C}(53)$ & $23(2)$ & $23(2)$ & $24(2)$ & $-6(2)$ & $-4(2)$ & $-1(2)$ \\
\hline $\mathrm{C}(54)$ & $34(2)$ & $19(2)$ & $16(2)$ & $-4(2)$ & $3(2)$ & $-1(2)$ \\
\hline $\mathrm{C}(55)$ & $24(2)$ & $24(2)$ & $24(2)$ & $-10(2)$ & $9(2)$ & $-5(2)$ \\
\hline$C(56)$ & $19(2)$ & $21(2)$ & $23(2)$ & $-11(2)$ & $0(2)$ & $0(2)$ \\
\hline $\mathrm{C}(57)$ & $17(2)$ & $14(2)$ & $14(2)$ & $-4(1)$ & $-2(1)$ & $0(1)$ \\
\hline $\mathrm{C}(58)$ & $10(2)$ & $14(2)$ & $18(2)$ & $-2(1)$ & $-1(1)$ & $-1(1)$ \\
\hline $\mathrm{C}(59)$ & $15(2)$ & $11(2)$ & $13(2)$ & $-4(1)$ & 1(1) & $0(1)$ \\
\hline $\mathrm{C}(60)$ & $14(2)$ & $13(2)$ & $17(2)$ & $-3(1)$ & $0(1)$ & $-4(1)$ \\
\hline $\mathrm{C}(61)$ & $13(2)$ & $17(2)$ & $19(2)$ & $-2(1)$ & $2(1)$ & $-2(1)$ \\
\hline $\mathrm{C}(62)$ & $15(2)$ & $17(2)$ & $17(2)$ & $-3(1)$ & $2(1)$ & $2(1)$ \\
\hline$C(63)$ & $12(2)$ & $22(2)$ & $17(2)$ & $3(2)$ & $0(1)$ & $-3(1)$ \\
\hline $\mathrm{C}(64)$ & $28(2)$ & $28(2)$ & $23(2)$ & $2(2)$ & $-8(2)$ & $-12(2)$ \\
\hline$C(65)$ & $35(3)$ & $37(3)$ & $29(2)$ & $11(2)$ & $-11(2)$ & $-19(2)$ \\
\hline$C(66)$ & $27(2)$ & $60(3)$ & $21(2)$ & $18(2)$ & $-1(2)$ & $-11(2)$ \\
\hline $\mathrm{C}(67)$ & $32(3)$ & $59(3)$ & $19(2)$ & $-1(2)$ & $7(2)$ & $4(2)$ \\
\hline $\mathrm{C}(68)$ & $26(2)$ & $34(2)$ & $20(2)$ & $-1(2)$ & $3(2)$ & $3(2)$ \\
\hline $\mathrm{C}(69)$ & $14(2)$ & $9(2)$ & $19(2)$ & $-5(1)$ & $-1(1)$ & $-2(1)$ \\
\hline$C(70)$ & $16(2)$ & $12(2)$ & $22(2)$ & $-2(1)$ & $2(2)$ & $-1(1)$ \\
\hline $\mathrm{C}(71)$ & $13(2)$ & $16(2)$ & $34(2)$ & $-5(2)$ & $5(2)$ & $-3(1)$ \\
\hline$C(72)$ & $15(2)$ & $13(2)$ & $38(2)$ & $-9(2)$ & $-8(2)$ & 1(1) \\
\hline$C(73)$ & $22(2)$ & $13(2)$ & $21(2)$ & $-8(1)$ & $-3(2)$ & $0(1)$ \\
\hline$C(74)$ & $15(2)$ & $9(2)$ & $21(2)$ & $-5(1)$ & $3(1)$ & $-4(1)$ \\
\hline $\mathrm{N}(1)$ & $25(2)$ & $26(2)$ & $20(2)$ & $-6(1)$ & 1(1) & $0(1)$ \\
\hline$C(75)$ & $32(3)$ & $63(4)$ & $33(3)$ & $-20(2)$ & $1(2)$ & $4(2)$ \\
\hline$C(76)$ & $56(3)$ & $41(3)$ & 41(3) & $-24(2)$ & $-19(3)$ & 21(3) \\
\hline$C(77)$ & $59(3)$ & $22(2)$ & $34(3)$ & $-5(2)$ & $-13(2)$ & $0(2)$ \\
\hline$C(78)$ & $28(2)$ & $25(2)$ & $26(2)$ & $-6(2)$ & $-2(2)$ & $-3(2)$ \\
\hline$C(79)$ & $30(2)$ & $33(2)$ & $22(2)$ & $-6(2)$ & $-5(2)$ & $-2(2)$ \\
\hline $\mathrm{C}(80)$ & $41(3)$ & $30(2)$ & $29(2)$ & $-13(2)$ & $3(2)$ & $-9(2)$ \\
\hline$C(81)$ & $46(3)$ & $28(2)$ & $38(3)$ & $-7(2)$ & $4(2)$ & $-3(2)$ \\
\hline$C(82)$ & $34(2)$ & $29(2)$ & $27(2)$ & $2(2)$ & $0(2)$ & $-8(2)$ \\
\hline $\mathrm{N}(2)$ & $23(2)$ & $18(2)$ & $29(2)$ & $-5(1)$ & 1(1) & $0(1)$ \\
\hline C(83) & $28(2)$ & $20(2)$ & $25(2)$ & $-9(2)$ & $0(2)$ & $2(2)$ \\
\hline C(84) & $43(3)$ & $30(2)$ & $37(3)$ & $-13(2)$ & $13(2)$ & $-7(2)$ \\
\hline$C(85)$ & $61(4)$ & $44(3)$ & $42(3)$ & $-18(2)$ & $22(3)$ & $-6(3)$ \\
\hline$C(86)$ & $33(2)$ & $35(2)$ & $24(2)$ & $-13(2)$ & $0(2)$ & $6(2)$ \\
\hline$C(87)$ & $25(2)$ & $21(2)$ & $28(2)$ & $-8(2)$ & $-2(2)$ & $1(2)$ \\
\hline C(88) & $36(2)$ & $23(2)$ & $32(2)$ & $-11(2)$ & $9(2)$ & $-7(2)$ \\
\hline C(89) & $25(2)$ & $28(2)$ & $59(3)$ & $-19(2)$ & $4(2)$ & $-9(2)$ \\
\hline $\mathrm{C}(90)$ & $27(2)$ & $32(2)$ & $46(3)$ & $-21(2)$ & $-9(2)$ & $-3(2)$ \\
\hline $\mathrm{N}(3)$ & $22(2)$ & $23(2)$ & $25(2)$ & $-13(1)$ & $-2(1)$ & $2(1)$ \\
\hline $\mathrm{C}(91)$ & $31(2)$ & $20(2)$ & $26(2)$ & $-8(2)$ & $-7(2)$ & $0(2)$ \\
\hline $\mathrm{C}(92)$ & $20(2)$ & $23(2)$ & $35(2)$ & $-9(2)$ & $-2(2)$ & $-2(2)$ \\
\hline C(93) & $25(2)$ & $28(2)$ & $24(2)$ & $-5(2)$ & $0(2)$ & $0(2)$ \\
\hline $\mathrm{C}(94)$ & $24(2)$ & $26(2)$ & $27(2)$ & $-6(2)$ & $-5(2)$ & $0(2)$ \\
\hline
\end{tabular}




$\begin{array}{lcccccc}\mathrm{C}(95) & 27(2) & 22(2) & 33(2) & -7(2) & -3(2) & 4(2) \\ \mathrm{C}(96) & 25(2) & 28(2) & 29(2) & -9(2) & -2(2) & 1(2) \\ \mathrm{C}(97) & 31(2) & 31(2) & 28(2) & -6(2) & 3(2) & -3(2) \\ \mathrm{C}(98) & 28(2) & 24(2) & 29(2) & -4(2) & 1(2) & -6(2) \\ \mathrm{Br}(1) & 28(1) & 21(1) & 25(1) & -8(1) & -5(1) & -3(1) \\ \mathrm{C}(102) & 82(6) & 73(5) & 94(6) & 21(5) & -18(5) & -28(4) \\ \mathrm{C}(101) & 161(12) & 57(4) & 66(5) & -27(4) & -47(7) & 17(6) \\ \mathrm{C}(100) & 1400(130) & 139(14) & 77(10) & -60(12) & -170(30) & 330(40) \\ \mathrm{O}(1) & 173(13) & 620(30) & 600(30) & -540(30) & -78(16) & 87(16) \\ \mathrm{C}(99) & 490(40) & 2000(130) & 360(30) & -860(60) & -280(30) & 760(60)\end{array}$


Table 20. Hydrogen coordinates $\left(\mathrm{x} 10^{4}\right)$ and isotropic displacement parameters $\left(\AA^{2} \times 10\right.$ 3 ) for $\{\mathrm{ASN}\}_{2}\left\{\left[\mathrm{Ph}_{3} \mathrm{BP}^{m-\mathrm{Ph} 2}\right]_{2} \mathrm{PtMe}_{2}\right\}$.

\begin{tabular}{|c|c|c|c|c|}
\hline & $\mathrm{x}$ & $\mathrm{y}$ & z & $\mathrm{U}(\mathrm{eq})$ \\
\hline $\mathrm{H}(1 \mathrm{~A})$ & 11814 & 10181 & 3656 & 36 \\
\hline $\mathrm{H}(1 \mathrm{~B})$ & 10416 & 10712 & 3637 & 36 \\
\hline $\mathrm{H}(1 \mathrm{C})$ & 11359 & 10755 & 3089 & 36 \\
\hline $\mathrm{H}(2 \mathrm{~A})$ & 12503 & 8875 & 3565 & 28 \\
\hline $\mathrm{H}(2 \mathrm{~B})$ & 11534 & 8192 & 3713 & 28 \\
\hline $\mathrm{H}(2 \mathrm{C})$ & 11512 & 8809 & 4100 & 28 \\
\hline $\mathrm{H}(4)$ & 7691 & 12453 & -177 & 43 \\
\hline $\mathrm{H}(5)$ & 7879 & 12242 & -1068 & 53 \\
\hline $\mathrm{H}(6)$ & 6690 & 11311 & -1304 & 43 \\
\hline $\mathrm{H}(7)$ & 5277 & 10614 & -641 & 40 \\
\hline $\mathrm{H}(8)$ & 5149 & 10791 & 263 & 33 \\
\hline $\mathrm{H}(10)$ & 5369 & 10346 & 1242 & 30 \\
\hline $\mathrm{H}(11)$ & 3424 & 9981 & 1722 & 30 \\
\hline $\mathrm{H}(12)$ & 1805 & 10942 & 1953 & 29 \\
\hline $\mathrm{H}(13)$ & 2158 & 12263 & 1666 & 28 \\
\hline $\mathrm{H}(14)$ & 4099 & 12634 & 1174 & 23 \\
\hline $\mathrm{H}(16)$ & 5229 & 13219 & 278 & 57 \\
\hline $\mathrm{H}(17)$ & 5628 & 14518 & 266 & 70 \\
\hline $\mathrm{H}(18)$ & 7399 & 14778 & 705 & 65 \\
\hline $\mathrm{H}(19)$ & 8712 & 13731 & 1221 & 56 \\
\hline $\mathrm{H}(20)$ & 8255 & 12445 & 1280 & 39 \\
\hline $\mathrm{H}(22)$ & 6960 & 11333 & 1885 & 18 \\
\hline $\mathrm{H}(24)$ & 10162 & 9705 & 1979 & 18 \\
\hline $\mathrm{H}(25)$ & 10193 & 9852 & 1022 & 22 \\
\hline $\mathrm{H}(26)$ & 8601 & 10713 & 502 & 22 \\
\hline $\mathrm{H}(28)$ & 9730 & 11740 & 2032 & 25 \\
\hline $\mathrm{H}(29)$ & 10021 & 13032 & 2057 & 30 \\
\hline $\mathrm{H}(30)$ & 9243 & 13538 & 2833 & 31 \\
\hline $\mathrm{H}(31)$ & 8182 & 12736 & 3576 & 30 \\
\hline $\mathrm{H}(32)$ & 7887 & 11447 & 3549 & 25 \\
\hline $\mathrm{H}(34)$ & 5769 & 10461 & 2440 & 21 \\
\hline $\mathrm{H}(35)$ & 3778 & 10244 & 2960 & 25 \\
\hline $\mathrm{H}(36)$ & 3735 & 9987 & 3919 & 28 \\
\hline $\mathrm{H}(37)$ & 5702 & 9915 & 4374 & 27 \\
\hline $\mathrm{H}(38)$ & 7709 & 10072 & 3862 & 23 \\
\hline $\mathrm{H}(40)$ & 7960 & 7235 & 1866 & 22 \\
\hline $\mathrm{H}(41)$ & 5816 & 7440 & 1551 & 28 \\
\hline $\mathrm{H}(42)$ & 4779 & 6387 & 1400 & 31 \\
\hline $\mathrm{H}(43)$ & 5940 & 5126 & 1572 & 31 \\
\hline $\mathrm{H}(44)$ & 8062 & 4913 & 1899 & 25 \\
\hline $\mathrm{H}(46)$ & 10900 & 6433 & 1166 & 27 \\
\hline $\mathrm{H}(47)$ & 12271 & 5910 & 526 & 34 \\
\hline $\mathrm{H}(48)$ & 12986 & 4551 & 703 & 37 \\
\hline $\mathrm{H}(49)$ & 12227 & 3742 & 1517 & 36 \\
\hline $\mathrm{H}(50)$ & 10877 & 4275 & 2164 & 28 \\
\hline $\mathrm{H}(52)$ & 11695 & 5035 & 2888 & 24 \\
\hline $\mathrm{H}(53)$ & 11695 & 4159 & 3739 & 28 \\
\hline
\end{tabular}




\begin{tabular}{|c|c|c|c|c|}
\hline $\mathrm{H}(54)$ & 9672 & 3823 & 4206 & 28 \\
\hline $\mathrm{H}(55)$ & 7678 & 4363 & 3797 & 28 \\
\hline $\mathrm{H}(56)$ & 7691 & 5235 & 2942 & 24 \\
\hline $\mathrm{H}(58)$ & 8785 & 7052 & 2698 & 17 \\
\hline $\mathrm{H}(60)$ & 11922 & 8297 & 2675 & 17 \\
\hline $\mathrm{H}(61)$ & 13219 & 7432 & 2208 & 20 \\
\hline $\mathrm{H}(62)$ & 12306 & 6407 & 1978 & 20 \\
\hline $\mathrm{H}(64)$ & 9203 & 6658 & 3617 & 31 \\
\hline $\mathrm{H}(65)$ & 8645 & 5914 & 4490 & 42 \\
\hline $\mathrm{H}(66)$ & 8108 & 6522 & 5232 & 47 \\
\hline $\mathrm{H}(67)$ & 8239 & 7865 & 5112 & 47 \\
\hline $\mathrm{H}(68)$ & 8778 & 8618 & 4240 & 34 \\
\hline $\mathrm{H}(70)$ & 6542 & 8170 & 3620 & 20 \\
\hline $\mathrm{H}(71)$ & 4453 & 8292 & 3258 & 26 \\
\hline $\mathrm{H}(72)$ & 4224 & 8642 & 2309 & 26 \\
\hline $\mathrm{H}(73)$ & 6104 & 8885 & 1709 & 22 \\
\hline $\mathrm{H}(74)$ & 8198 & 8755 & 2070 & 17 \\
\hline $\mathrm{H}(75 \mathrm{~A})$ & 1034 & 2597 & 10704 & 50 \\
\hline $\mathrm{H}(75 \mathrm{~B})$ & -179 & 2624 & 10317 & 50 \\
\hline $\mathrm{H}(76 \mathrm{~A})$ & 921 & 3895 & 10316 & 54 \\
\hline $\mathrm{H}(76 \mathrm{~B})$ & 266 & 3789 & 9771 & 54 \\
\hline $\mathrm{H}(77 \mathrm{~A})$ & 2286 & 3721 & 9345 & 46 \\
\hline $\mathrm{H}(77 \mathrm{~B})$ & 2940 & 3822 & 9891 & 46 \\
\hline $\mathrm{H}(78 \mathrm{~A})$ & 3388 & 2504 & 9549 & 32 \\
\hline $\mathrm{H}(78 \mathrm{~B})$ & 3424 & 2510 & 10194 & 32 \\
\hline $\mathrm{H}(79 \mathrm{~A})$ & 1169 & 2655 & 9167 & 34 \\
\hline $\mathrm{H}(79 \mathrm{~B})$ & 61 & 2131 & 9515 & 34 \\
\hline $\mathrm{H}(80 \mathrm{~A})$ & 2663 & 1556 & 9145 & 38 \\
\hline $\mathrm{H}(80 \mathrm{~B})$ & 1274 & 1253 & 9052 & 38 \\
\hline $\mathrm{H}(81 \mathrm{~A})$ & 1077 & 565 & 9942 & 45 \\
\hline $\mathrm{H}(81 \mathrm{~B})$ & 2672 & 497 & 9873 & 45 \\
\hline $\mathrm{H}(82 \mathrm{~A})$ & 2777 & 1268 & 10459 & 37 \\
\hline $\mathrm{H}(82 \mathrm{~B})$ & 1210 & 1224 & 10596 & 37 \\
\hline $\mathrm{H}(83 \mathrm{~A})$ & 3188 & 3803 & 2765 & 29 \\
\hline $\mathrm{H}(83 \mathrm{~B})$ & 3900 & 3632 & 3344 & 29 \\
\hline $\mathrm{H}(84 \mathrm{~A})$ & 5972 & 3812 & 2910 & 44 \\
\hline $\mathrm{H}(84 \mathrm{~B})$ & 5029 & 4489 & 2523 & 44 \\
\hline $\mathrm{H}(85 \mathrm{~A})$ & 4883 & 3704 & 1892 & 59 \\
\hline $\mathrm{H}(85 \mathrm{~B})$ & 6421 & 3514 & 2042 & 59 \\
\hline $\mathrm{H}(86 \mathrm{~A})$ & 6102 & 2335 & 2586 & 36 \\
\hline $\mathrm{H}(86 \mathrm{~B})$ & 4893 & 2394 & 2191 & 36 \\
\hline $\mathrm{H}(87 \mathrm{~A})$ & 5419 & 2496 & 3630 & 29 \\
\hline $\mathrm{H}(87 \mathrm{~B})$ & 5236 & 1697 & 3441 & 29 \\
\hline $\mathrm{H}(88 \mathrm{~A})$ & 3463 & 2479 & 4122 & 36 \\
\hline $\mathrm{H}(88 \mathrm{~B})$ & 3595 & 1560 & 4106 & 36 \\
\hline $\mathrm{H}(89 \mathrm{~A})$ & 1805 & 1814 & 3590 & 43 \\
\hline $\mathrm{H}(89 \mathrm{~B})$ & 1802 & 2747 & 3538 & 43 \\
\hline $\mathrm{H}(90 \mathrm{~A})$ & 3165 & 1822 & 2818 & 39 \\
\hline $\mathrm{H}(90 \mathrm{~B})$ & 2405 & 2695 & 2646 & 39 \\
\hline $\mathrm{H}(91 \mathrm{~A})$ & 5203 & 5269 & 4383 & 30 \\
\hline $\mathrm{H}(91 \mathrm{~B})$ & 4885 & 6111 & 4549 & 30 \\
\hline $\mathrm{H}(92 \mathrm{~A})$ & 6626 & 5885 & 3740 & 30 \\
\hline $\mathrm{H}(92 \mathrm{~B})$ & 5903 & 6743 & 3771 & 30 \\
\hline $\mathrm{H}(93 \mathrm{~A})$ & 4696 & 6764 & 3047 & 31 \\
\hline $\mathrm{H}(93 \mathrm{~B})$ & 5544 & 5955 & 2973 & 31 \\
\hline $\mathrm{H}(94 \mathrm{~A})$ & 3027 & 5972 & 3220 & 31 \\
\hline
\end{tabular}




$\begin{array}{lrrrr}\mathrm{H}(94 \mathrm{~B}) & 4007 & 5200 & 3467 & 31 \\ \mathrm{H}(95 \mathrm{~A}) & 2091 & 6864 & 3753 & 34 \\ \mathrm{H}(95 \mathrm{~B}) & 3428 & 7134 & 3931 & 34 \\ \mathrm{H}(96 \mathrm{~A}) & 1304 & 6954 & 4613 & 33 \\ \mathrm{H}(96 \mathrm{~B}) & 2773 & 6878 & 4836 & 33 \\ \mathrm{H}(97 \mathrm{~A}) & 2733 & 5573 & 5154 & 36 \\ \mathrm{H}(97 \mathrm{~B}) & 1218 & 5662 & 4979 & 36 \\ \mathrm{H}(98 \mathrm{~A}) & 1801 & 5343 & 4162 & 33 \\ \mathrm{H}(98 \mathrm{~B}) & 3027 & 4852 & 4505 & 33 \\ \mathrm{H}(10 \mathrm{~A}) & 4417 & 8304 & 4328 & 130 \\ \mathrm{H}(10 \mathrm{~B}) & 4842 & 7607 & 4841 & 130 \\ \mathrm{H}(10 \mathrm{C}) & 5489 & 8421 & 4735 & 130 \\ \mathrm{H}(10 \mathrm{D}) & 2921 & 8149 & 5022 & 111 \\ \mathrm{H}(10 \mathrm{E}) & 3963 & 8246 & 5425 & 111 \\ \mathrm{H}(10 \mathrm{~F}) & 226 & 10414 & 5012 & 837 \\ \mathrm{H}(10 \mathrm{G}) & 1408 & 10581 & 4553 & 837 \\ \mathrm{H}(10 \mathrm{H}) & 1593 & 10659 & 5170 & 837 \\ \mathrm{H}(99 \mathrm{~A}) & 1264 & 9329 & 4821 & 1095 \\ \mathrm{H}(99 \mathrm{~B}) & 1220 & 9369 & 5443 & 1095\end{array}$

